\title{
THE INFLUENCE OF THE COASTAL FRONT ON HEAVY RAINFALL EVENTS ALONG THE EAST COAST
}

\author{
A Dissertation Presented to the Faculty of the Graduate School at the \\ University of Missouri
}

In Partial Fulfillment of the Requirements for the Degree

Doctor of Philosophy

by

CHASITY B. HENSON

Dr. Patrick Market, Dissertation Advisor

DECEMBER 2019 
The undersigned, appointed by the dean of the Graduate School, have examined the dissertation entitled

THE INFLUENCE OF THE COASTAL FRONT ON HEAVY RAINFALL EVENTS ALONG THE EAST COAST

presented by Chasity B. Henson, a candidate for the degree of doctor of philosophy, and hereby certify that, in their opinion, it is worthy of acceptance.

Director Patrick Market

Professor Anthony Lupo

Professor Neil Fox

Professor Mark Palmer

Director Chris Davis 


\section{ACKNOWLEDGEMENTS}

My deepest gratitude is expressed to Dr. Patrick Market for being my advisor, professor, and biggest supporter. This research would not have been possible without the guidance from Dr. Market to help shape the objectives and methodology. Dr. Market was there to answer my all of questions and review my scientific explanations. He has always been encouraging, from the moment he met me and asked me to join the program at the University of Missouri. That encouragement and his exceptional guidance is the reason I am a successful meteorologist.

I thank my committee members, especially my outside member Dr. Mark Palmer, for taking the time to assess my performance as an atmospheric science doctoral student. In addition to all the skills I acquired completing this dissertation research, the knowledge I gained through the atmospheric science classes at the University of Missouri shaped me into the professional meteorologist I am today. For that, I thank my graduate professors: Patrick Market, Anthony Lupo, and Neil Fox.

The National Center for Atmospheric Research (NCAR) is acknowledged, specifically Dr. Chris Davis and Dr. Wei Wang, for recognizing my research and inviting me to NCAR's Mesoscale and Microscale Meteorology (MMM) Laboratory for a summer of collaboration. Dr. Wang taught me several important concepts involved in the configuration of the Weather Research and Forecasting (WRF) model, both in the technical and meteorological aspects, that strengthened the methodology of this research. WRF model simulations were completed using NCAR's high-performance computer, 
Cheyenne (doi:10.5065/D6RX99HX), provided by NCAR's Computational and Information Systems Laboratory (CISL) and sponsored by the National Science Foundation. Dr. Davis provided guidance for the dissertation objectives, helped me understand complex atmospheric concepts, and served as a member on my dissertation committee.

Throughout my Ph.D. program, I participated in several graduate student opportunities, from seminars to internships, across the country. I am grateful for the relationships I made along the way. The support from fellow graduate students in atmospheric science from several different universities helped motivate me to finish the degree and obtain my dream career. I also gained technical skills and meteorological knowledge from my fellow graduate students and my mentors at both NCAR and the Naval Research Laboratory (NRL). Dr. Alicia Bentley shared her code for irrotational wind and produced images for me, which I later recreated for this research (Fig. 4.2). Dr. William Komaromi and Dr. James Doyle gave me the opportunity to work at NRL as an intern and I successfully contributed to their research objectives. The skills and friendships I gained, through all of the amazing opportunities I had, are a part of my daily life and contribute to my success as a meteorologist.

I must also acknowledge my employer ENSCO, Inc. for supporting me through the final stages of my Ph.D. My coworkers helped me reach my goals by constantly asking for updates near the end of my Ph.D. program to hold me accountable to the deadlines I set for myself. Primary support of this research came from Missouri EPSCoR (Experimental Program to Stimulate Competitive Research), University of Missouri PRECIP (Program for Research of Elevated Convection with Intense Precipitation), and 
the University of Missouri Research Board. With EPSCoR and PRECIP being chapters of the National Science Foundation, the official disclaimer is as follows:

This material is based upon work supported by the National Science Foundation under Awards IIA-1355406 and AGS-1258358. Any opinions, findings, and conclusions or recommendations expressed in this material are those of the author(s) and do not necessarily reflect the views of the National Science Foundation.

Lastly, but most importantly, I acknowledge my boyfriend. David has been by my side every step of the way. Through classwork, qualifying exams, conferences, internships, countless trips to different parts of the country, several late nights, and three research theses, he was there to entertain me when I needed breaks, hold my hand when I was stressed, and celebrate with me when I reached milestones. I greatly appreciate his patience with me as I finished up the Ph.D. while working full time as a professional meteorologist. The last year brought a lot of changes to both of our lives, but we pushed through it together. I look forward to spending all of my newfound free time with him in our new home. 


\section{TABLE OF CONTENTS}

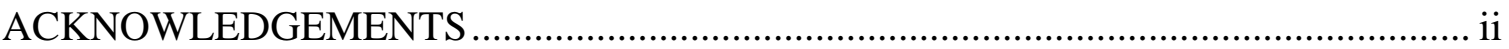

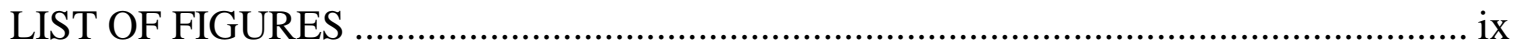

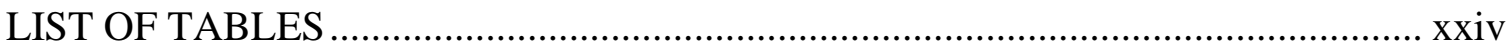

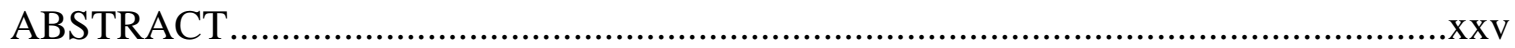

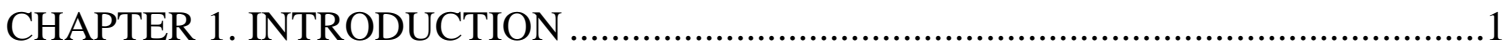

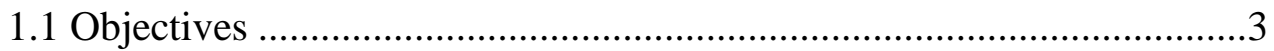

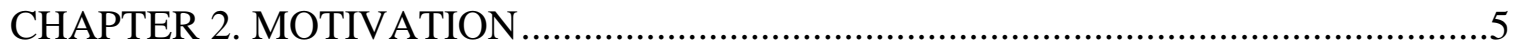

2.1 Literature Review..................................................................

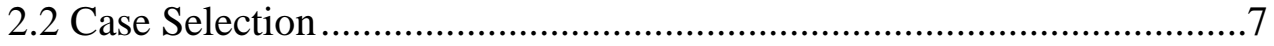

2.3 East Coast Extreme Rain ........................................................... 8

2.4 Observational Analysis ...............................................................12

2.4.1 2015 South Carolina Flood............................................12

2.4.1.1 Hurricane Joaquin ..................................12

2.4.1.2 Synoptic .............................................. 15

2.4.1.3 Mesoscale...............................................17

2.4.1.4 Forecast and Impacts..............................19

2.4.2 September 2011 Flood...................................................22

2.4.2.1 Hurricane Katia ........................................22

2.4.2.2 Pattern for Precipitation ............................25 
2.4.4 August 2014 - New York..........................................29

2.4.5 September 2010 - North Carolina..................................31

2.5 Initial Motivation ..............................................................................34

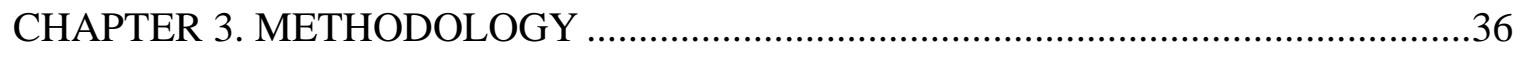

3.1 Numerical Modeling ...............................................................36

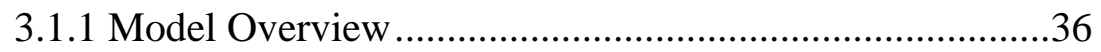

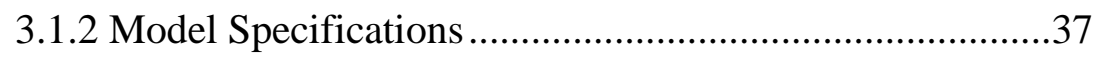

3.1.3 Experimental Simulations .........................................39

3.1.4 Model Verification ...................................................43

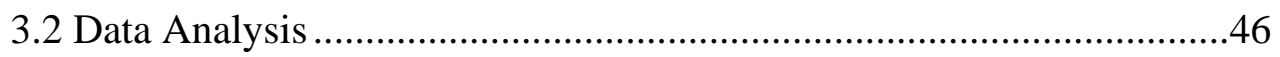

3.2.1 Synoptic Scale .......................................................4

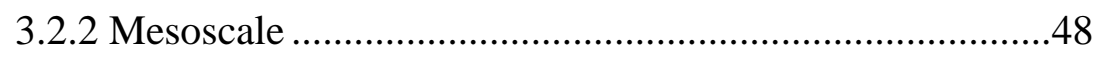

3.2.3 Parcel Trajectories ...................................................49

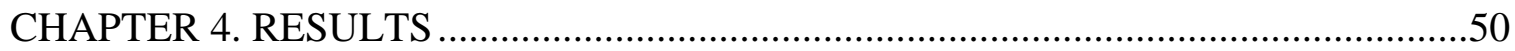

4.12015 South Carolina Flood ..........................................................50

4.1.1 Synoptic Scale .......................................................51

4.1.2 Mesoscale .................................................................56

4.1.3 Trajectories .............................................................66

4.1.4 Role of the Tropical Cyclone ......................................71

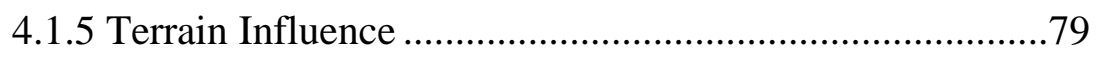

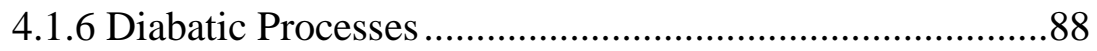

4.2 September 2011 Flood ............................................................98 
4.2.1 Synoptic Scale

4.2.2 Mesoscale .102

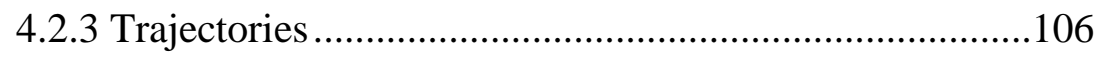

4.2.4 Role of the Tropical Cyclone ..........................................108

4.2.5 Terrain Influence .....................................................110

4.2.6 Diabatic Processes .........................................................113

4.3 September 2016 - North Carolina ………………………………......116

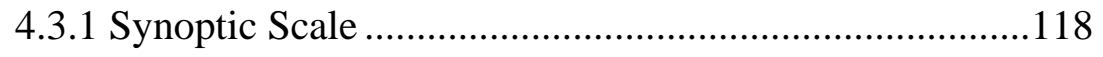

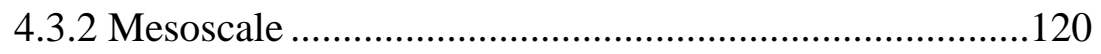

4.3.3 Terrain Influence .......................................................125

4.3.4 Diabatic Processes ..........................................................127

4.4 August 2014 - New York ……………………………………........129

4.4.1 Synoptic Scale ………………………………….........130

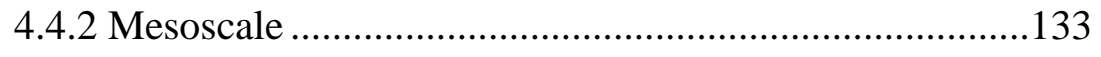

4.4.3 Terrain Influence ...........................................................137

4.4.4 Diabatic Processes ...........................................................138

4.5 September 2010 - North Carolina ………………………..................140

4.5.1 Synoptic Scale ……………………….........................141

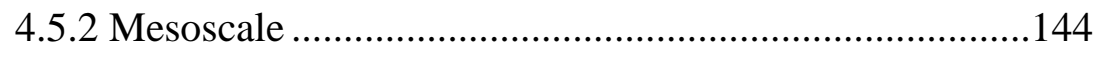

4.5.3 Terrain Influence ……………......................................149

4.5.4 Diabatic Processes ..........................................................152

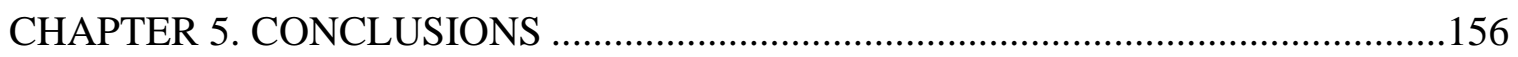

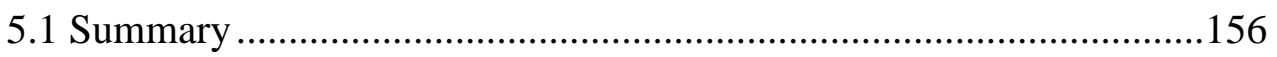




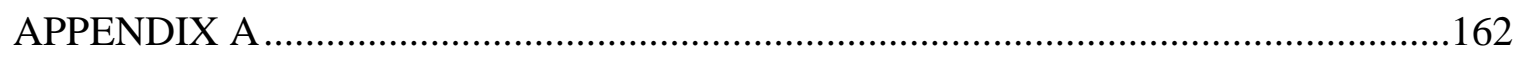

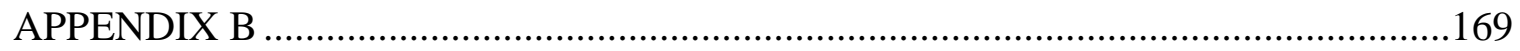

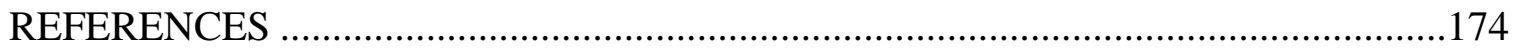

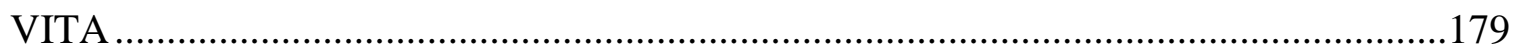




\section{LIST OF FIGURES}

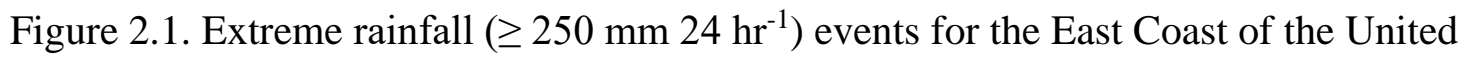
States (Florida excluded) from 2005 to 2017, excluding events associated with the landfall of a tropical cyclone. Each individual report was obtained from the Community Collaborative Rain, Hail and Snow (CoCoRaHS) Network and is identified by the marking of its associated event.

Figure 2.2. Hurricane Joaquin best track a) positions and rank, b) minimum central pressure, and c) maximum sustained surface wind speed covering the period of Joaquin's hurricane status from 28 September 2015 to 07 September 2015 (Berg 2016).

Figure 2.3. National Oceanic and Atmospheric Administration (NOAA) 1200 UTC 02 October 2015 a) 500-hPa analysis displaying an extratropical cyclone centered over Alabama with Hurricane Joaquin near the Bahamas and b) surface analysis depicting a stationary frontal boundary along the East Coast of the United States.

Figure 2.4. Visible satellite images from NOAA's GOES-East of Hurricane Joaquin and the associated moisture plume directed towards South Carolina at a) 1200 UTC 03 October 2015 and b) 1200 UTC 05 October 2015.........................15

Figure 2.5. As in Fig. 2.3, except 1200 UTC 05 October 2015...................................16

Figure 2.6. Radar reflectivity (dBZ) mosaic from College of DuPage Next Generation Weather Lab over the southeastern United States at a) 1225 UTC 03 October 2015 and b) 1155 UTC 04 October 2015

Figure 2.7. Observed sounding from Charleston, South Carolina National Weather Service (NWS) at 0000 UTC 04 October 2015 obtained by The Universal Rawinsonde Observation program (RAOB).

Figure 2.8. Total observed precipitation (inches) for South Carolina from 0000 UTC 01 October 2015 to 1100 UTC 05 October 2015. Image courtesy of NWS offices in South Carolina and North Carolina.

Figure 2.9. Image of the flood waters near Columbia, South Carolina as a result of the heavy rainfall in early October 2015. Image from the NWS Columbia, SC Event Review of the 2015 South Carolina Flood.

Figure 2.10. Hurricane Katia best track positions and rank from 29 August 2011 to 10 September 2011 (Stewart 2012). 
Figure 2.11. Hurricane Katia best track a) maximum sustained surface wind speed and b) minimum central pressure from 28 August 2011 to 13 September 2011 (Stewart 2012).

Figure 2.12. NOAA 1200 UTC 08 September 2011 a) 500-hPa analysis displaying an extratropical cyclone centered over the Ohio River Valley with Hurricane Katia near Bermuda and b) surface analysis depicting a surface low and associated frontal boundary along the East Coast of the United States.

Figure 2.13. Northeastern United States a) visible satellite image from NOAA's GOESEast of Hurricane Katia and the moisture plume directed towards Pennsylvania at 1400 UTC 07 September 2011, b) NWS radar reflectivity (dBZ) mosaic at 0438 UTC 08 September 2011, and c) NWS derived total rainfall (inches) for the 24-hour event from 1200 UTC 07 September 2011 to 1200 UTC 08 September 2011.

Figure 2.14. NOAA 1200 UTC 29 September 2016 a) 500-hPa analysis displaying an extratropical cyclone centered over the Ohio River Valley and b) surface analysis depicting a surface low and associated frontal boundary along the East Coast of the United States.

Figure 2.15. East Coast United States a) visible satellite image from NOAA's GOES-East at 2215 UTC 28 September 2016 and b) radar reflectivity (dBZ) mosaic from College of DuPage Next Generation Weather Lab at 0425 UTC 29 September 2016 depicting convection over North Carolina and Virginia. ....28

Figure 2.16. NOAA a) 0000 UTC 13 August 2014 500-hPa analysis displaying a trough over the eastern United States and b) 0900 UTC 13 August 2014 surface analysis depicting a surface low and associated frontal boundaries along the East Coast of the United States.

Figure 2.17. Northeastern United States a) radar reflectivity (dBZ) mosaic from College of DuPage Next Generation Weather Lab at 0925 UTC 13 August 2014 and b) NWS radar estimated total precipitation (inches) from 0500 UTC 12 August 2014 to 1800 UTC 13 August 2014 showing a maximum total of 19 inches for Long Island, New York and large area with greater than 10 inches of rain.

Figure 2.18. NOAA a) 1200 UTC 30 September 2010 500-hPa analysis displaying an extratropical cyclone over Georgia, b) 0000 UTC 30 September 2010 surface analysis depicting a stationary front along the East Coast of the United States, and c) 1200 UTC 30 September 2010 surface analysis depicting a surface low and associated frontal boundaries in North Carolina. 
Figure 2.19. East Coast United States a) visible satellite image from NOAA's GOES-East at 1325 UTC 30 September 2010 and b) radar reflectivity (dBZ) mosaic from College of DuPage Next Generation Weather Lab at 1225 UTC 30

September 2010 showing a north-south oriented line of convection. .32

Figure 2.19. NWS derived total rainfall (inches) over the East Coast of the United States from 0000 UTC 29 September 2010 to 1200 UTC 02 October 2010 showing a maximum of 17.84 inches of rain near Swansboro, North Carolina in the 84-hour period.

Figure 2.20. The moisture transport associated with the 2015 South Carolina flood event was analyzed using a) the ARL HYSPLIT model to create 72-hour backward trajectories of air parcels located over South Carolina at 0000 UTC 04 October 2015, which was a time of heavy rainfall for the state, and b) visible satellite imagery from NOAA's GOES-East satellite of Hurricane Joaquin and the associated moisture plume at 1200 UTC 03 October 2015.

Figure 3.1. WRF model domains representing the outer domain (domain 1) of 15-km grid spacing with the inner domain (d02) of 3-km grid spacing outlined in blue...39

Figure 3.2. Precipitation amount $(\mathrm{mm})$ for the South Carolina flood from 0000 UTC 01 October 2015 to 1200 UTC 05 October 2015 created using a) 4-km NCEP Stage IV reanalysis data from Earth Observing Laboratory (EOL) and b) WRF control simulation output for domain 1. The MODE results are shown to identify an object (red) for c) the reanalysis data shown in a) and d) the WRF data shown in b) that are corresponding in both analyses based on a threshold of $508 \mathrm{~mm}$. The total interest value calculated in MODE for the objects (red) shown in c) and d) is 0.96 .......

Figure 3.3. As in Fig. 3.2, except for domain 2 with a total interest value of 0.93 . .44

Figure 3.4. Hurricane Joaquin a) tracks and b) central pressures from 0000 UTC 01 October 2015 to 1200 UTC 05 October 2015 resulting from the National Hurricane Center best track analysis (black) and WRF d01 control simulation (red).

Figure 4.1. Precipitation (mm) for the South Carolina flood event from 0000 UTC 01 October 2015 to 1200 UTC 05 October 2015 for domain 2 of the WRF control simulation a) at the location of maximum precipitation, plotted as accumulation with time and b) as the event total for the region, with the white star (south) representing Mount Pleasant, South Carolina and the transparent star (north) representing the location of heaviest rainfall near Florence, South Carolina. 
Figure 4.2. Climate Forecast System Reanalysis (CFSR) valid at a) 1200 UTC 03 October 2015 and b) 0600 UTC 04 October 2015 representing 250-hPa wind ( $\mathrm{m} \mathrm{s}^{-1}$; shaded), 600-400-hPa ascent $\left(5 \times 10^{-3} \mathrm{hPa} \mathrm{s}^{-1}\right.$; dashed red), 300-200-hPa potential vorticity (PVU; contoured) and irrotational wind $\left(\mathrm{m} \mathrm{s}^{-1}\right)$.

Figure 4.3. WRF control simulation 300-hPa height (gpm; thick contoured), wind ( $\mathrm{m} \mathrm{s}^{-1}$; shaded; thin streamlines), and divergence $\left(10^{-5} \mathrm{~s}^{-1}\right.$; contoured; positive red) at a) 1200 UTC 03 October 2015 and b) 1200 UTC 04 October 2015 for domain 1 .

Figure 4.4. WRF results for domain 1 of the control simulation at a) 1200 UTC 03 October 2015 and b) 1200 UTC 04 October 2015 displaying precipitable water (mm; shaded), sea level pressure (hPa; contoured), and 850-hPa wind $\left(\mathrm{m} \mathrm{s}^{-1}\right)$

Figure 4.5. WRF results for domain 1 of the control simulation at a) 1200 UTC 03 October 2015 and b) 1200 UTC 04 October 2015 displaying integrated water vapor transport (IVT; $\mathrm{kg} \mathrm{m}^{-1} \mathrm{~s}^{-1}$ ).

Figure 4.6. WRF results for domain 2 of the control simulation at 1200 UTC 03 October 2015 displaying sea level pressure (hPa; contoured), 2-m temperature $\left({ }^{\circ} \mathrm{C}\right.$; shaded), and 10-m wind ( $\left.\mathrm{m} \mathrm{s}^{-1}\right)$.

Figure 4.7. WRF results for domain 2 of the control simulation at 1200 UTC 03 October 2015 displaying a) 2-m temperature $\left({ }^{\circ} \mathrm{C}\right.$; dashed), 950-hPa reflectivity (dBZ; shaded), 10-m wind $\left(\mathrm{m} \mathrm{s}^{-1}\right)$ and $\left.\mathrm{b}\right)$ potential temperature ( $\mathrm{K}$; contoured), reflectivity (dBZ; shaded), and circulation vectors $\left(\mathrm{m} \mathrm{s}^{-1}, \mathrm{dPa} \mathrm{s}^{-1}\right)$. Line segment $A B$ in a) represents the location of $b$ ).

Figure. 4.8. WRF domain 2 control simulation at 1200 UTC 03 October 2015 representing a) 700-hPa horizontal advective, b) 900-hPa vertical advective, c) 950-hPa tilting, d) 1000-hPa deformation, e) 1000-hPa divergence, and f) 700$\mathrm{hPa}$ diabatic frontogenesis (positive red, negative blue) contoured every $50 \mathrm{~K}$ $\left(100 \mathrm{~km} \mathrm{~h}^{-1}\right.$ for all except tilting and diabatic terms, which are contoured every $100 \mathrm{~K}\left(100 \mathrm{~km} \mathrm{~h}^{-1}\right.$. Line segment $\mathrm{CD}$ in $\left.\mathrm{d}\right)$ represents the location of future cross sections.

Figure 4.9. Cross sections CD (Fig. 4.8d) showing a) horizontal advective, b) vertical advective, c) tilting, d) deformation, e) divergence, and f) diabatic frontogenesis [K $(100 \mathrm{~km} \mathrm{~h})^{-1}$; shaded; positive red, negative blue], potential temperature (K; contoured), and circulation vectors $\left[\mathrm{m} \mathrm{s}^{-1}, \mathrm{dPa} \mathrm{s}^{-1}\left(1 \mathrm{dPa} \mathrm{s}^{-1}=\right.\right.$ $\left.10^{-3} \mathrm{mb} \mathrm{s}^{-1}\right)$ ] at 1200 UTC 03 October 2015 from WRF domain 2 .

Figure 4.10. WRF results for domain 2 of the control simulation at 1200 UTC 03 October 2015 displaying 850-hPa height (gpm; contoured) and wind $\left(\mathrm{m} \mathrm{s}^{-1}\right)$..... 
Figure 4.11. Cross section CD (Fig. 4.8d) at 1200 UTC 03 October 2015 showing equivalent potential temperature ( $\mathrm{K}$; contoured), relative humidity (shaded), and circulation vectors $\left[\mathrm{m} \mathrm{s}^{-1}, \mathrm{dPa} \mathrm{s}^{-1}\left(1 \mathrm{dPa} \mathrm{s}^{-1}=10^{-3} \mathrm{mb} \mathrm{s}^{-1}\right)\right]$ from WRF domain 2 .

Figure 4.12. WRF results for domain 2 of the control simulation at 0000 UTC 05 October 2015 displaying a) 850-hPa height (gpm; contoured), 850-hPa wind ( $\left.\mathrm{m} \mathrm{s}^{-1}\right)$, and b) 2-m temperature $\left({ }^{\circ} \mathrm{C}\right.$; dashed), 950-hPa reflectivity (dBZ; shaded), and $10-\mathrm{m}$ wind $\left(\mathrm{m} \mathrm{s}^{-1}\right)$.

Figure 4.13. WRF results for domain 2 of the control simulation at 0000 UTC 05 October 2015 displaying a) sea level pressure (hPa; contoured), 2-m temperature $\left({ }^{\circ} \mathrm{C}\right.$; shaded), and 10-m wind ( $\mathrm{m} \mathrm{s}^{-1}$ ), and b) cross section CD (Fig. 4.8d) representing diabatic frontogenesis [ $\mathrm{K}\left(100 \mathrm{~km} \mathrm{~h}^{-1}\right.$; shaded, positive red], potential temperature (K; contoured), and circulation vectors $\left[\mathrm{m} \mathrm{s}^{-1}, \mathrm{dPa} \mathrm{s}^{-1}\right.$ $\left.\left(1 \mathrm{dPa} \mathrm{s}^{-1}=10^{-3} \mathrm{mb} \mathrm{s}^{-1}\right)\right]$.

Figure 4.14. Parcel trajectories for the control simulation of the 2015 South Carolina flood event beginning at 1200 UTC 03 October 2015 in the region of the heaviest rainfall, point $\mathrm{C}$, and working backward six hours. The trajectories are colored by height above ground/pressure of the parcel at point $\mathrm{C}$. The arrows on the trajectory represent the height of the parcel at that time, with larger arrows representing a higher level.

Figure 4.15. The control simulation of the 2015 South Carolina flood event is represented by a) a parcel trajectory beginning at 1200 UTC 03 October 2015 in the region of the heaviest rainfall, point $\mathrm{C}$, at $820 \mathrm{mb}$ and working backward six hours. The arrows on the trajectory represent the height of the parcel at that time, with larger arrows representing a higher level. Points C and D (same as Fig. 4.8d) on the trajectory represent the region of the b) cross section at 1200 UTC 03 October 2015, which includes potential vorticity (PVU; red shaded), potential temperature (K; contoured), and circulation vectors $\left(\mathrm{m} \mathrm{s}^{-1}\right.$, $\mathrm{dPa} \mathrm{s}^{-1}$ ). Soundings for the parcel location along the trajectory were created in 5-minute intervals beginning with c) S1 at 1130 UTC, d) S2 at 1135 UTC, and e) S3 at 1140 UTC 03 October 2015 to represent the environment of the parcel before, during, and after crossing the frontal boundary, evident in b). Diagnostics for the parcel of interest are included with each sounding to represent the state of the parcel at that time.

Figure 4.16. Precipitation (mm) for the South Carolina flood event from 0000 UTC 01 October 2015 to 1200 UTC 05 October 2015 for domain 2 of the WRF simulation with the vortex of Hurricane Joaquin removed a) at the location of maximum precipitation, plotted as accumulation with time and b) as the event total for the region. The transparent star represents the location of maximum precipitation. 
Figure 4.17. WRF domain 1 300-hPa height (gpm; thick contoured), wind ( $\mathrm{m} \mathrm{s}^{-1}$; shaded; thin streamlines), and divergence $\left(10^{-5} \mathrm{~s}^{-1}\right.$; contoured; positive red) at a) 1200 UTC 03 October 2015 and b) 1200 UTC 04 October 2015 of the simulation with the removal of the tropical cyclone vortex. .73

Figure 4.18. WRF results for domain 1 of the simulation with the tropical cyclone vortex removed at a) 1200 UTC 03 October 2015 and b) 1200 UTC 04 October 2015 displaying precipitable water ( $\mathrm{mm}$; shaded), sea level pressure ( $\mathrm{hPa}$; contoured), and 850-hPa wind $\left(\mathrm{m} \mathrm{s}^{-1}\right)$.

Figure 4.19. WRF results for domain 1 of the simulation with the removal of the tropical cyclone vortex at a) 1200 UTC 03 October 2015 and b) 1200 UTC 04 October 2015 displaying integrated water vapor transport (IVT; $\mathrm{kg} \mathrm{m}^{-1} \mathrm{~s}^{-1}$ ).

Figure 4.20. WRF cross sections for domain 2 of the simulation with the tropical cyclone vortex removed at 1200 UTC 02 October 2015 for line segment CD (Fig. 4.8d) displaying potential temperature ( $\mathrm{K}$; contoured) and circulation vectors $\left[\mathrm{m} \mathrm{s}^{-1}, \mathrm{dPa} \mathrm{s}^{-1}\left(1 \mathrm{dPa} \mathrm{s}^{-1}=10^{-3} \mathrm{mb} \mathrm{s}^{-1}\right)\right]$ with a) potential vorticity (PVU; shaded red) and b) deformation frontogenesis [K $\left(100 \mathrm{~km} \mathrm{~h}^{-1}\right.$; shaded, positive red].

Figure 4.21. WRF results for domain 2 of the simulation with the tropical cyclone vortex removed at a) 1200 UTC 02 October 2015 and b) 1200 UTC 03 October 2015 displaying sea level pressure (hPa; contoured), 2-m temperature $\left({ }^{\circ} \mathrm{C}\right.$; shaded), 10-m wind $\left(\mathrm{m} \mathrm{s}^{-1}\right)$, and line segment EF.

Figure 4.22. WRF sounding from domain 2 of the simulation with the tropical cyclone vortex removed at 1200 UTC 03 October 2015 for the location of maximum precipitation showing temperature $\left({ }^{\circ} \mathrm{C}\right.$; red), dew point $\left({ }^{\circ} \mathrm{C}\right.$; blue $)$, and wind $\left(\mathrm{m} \mathrm{s}^{-1}\right)$.

Figure 4.23. Total accumulated precipitation ( $\mathrm{mm}$ ) from WRF domain 2 for the simulation with a) terrain removed and b) terrain doubled. .80

Figure 4.24. WRF domain 1 300-hPa height (gpm; thick contoured), wind ( $\mathrm{m} \mathrm{s}^{-1}$; shaded; thin streamlines), and divergence $\left(10^{-5} \mathrm{~s}^{-1}\right.$; contoured; positive red) at 1200 UTC 03 October 2015 for the simulation with a) terrain removed and b) terrain doubled.

Figure 4.25. WRF results for domain 1 at 1200 UTC 03 October 2015 displaying precipitable water ( $\mathrm{mm}$; shaded), sea level pressure (hPa; contoured), and $850-\mathrm{hPa}$ wind $\left(\mathrm{m} \mathrm{s}^{-1}\right)$ for the simulation with a) terrain removed and $\mathrm{b}$ ) terrain doubled. 
Figure 4.26. WRF results for domain 1 displaying a) the track of Hurricane Joaquin from 0000 UTC 01 October 2015 to 1200 UTC 05 October 2015 and b) precipitable water ( $\mathrm{mm}$; shaded), sea level pressure (hPa; contoured), and 850-hPa wind $\left(\mathrm{m} \mathrm{s}^{-1}\right)$ at 1200 UTC 05 October 2015 for the simulation with terrain removed.

Figure 4.27. WRF results for domain 2 at 1200 UTC 03 October 2015 displaying sea level pressure (hPa; contoured), 2-m temperature $\left({ }^{\circ} \mathrm{C}\right.$; shaded), 10-m wind ( $\mathrm{m}$ $\mathrm{s}^{-1}$ ), and line segment $\mathrm{GH}$ for the simulation with a) terrain removed and $\mathrm{b}$ ) terrain doubled.

Figure 4.28. WRF cross sections for domain 2 at 1200 UTC 03 October 2015 for line segment GH (Fig. 4.27a) displaying potential vorticity (PVU; shaded red), potential temperature $\left(\mathrm{K}\right.$; contoured), and circulation vectors $\left[\mathrm{m} \mathrm{s}^{-1}, \mathrm{dPa} \mathrm{s}^{-1}\right.$ $\left.\left(1 \mathrm{dPa} \mathrm{s}^{-1}=10^{-3} \mathrm{mb} \mathrm{s}^{-1}\right)\right]$ for the simulation with a) terrain removed and $\left.\mathrm{b}\right)$ terrain doubled.

Figure 4.29. WRF domain 2 cross sections for line segment CD (Fig. 4.8d) at 1200 UTC 03 October 2015 displaying potential temperature (K; contoured) and circulation vectors $\left(\mathrm{m} \mathrm{s}^{-1}, \mathrm{dPa} \mathrm{s}^{-1}\right)$ with potential vorticity (PVU; shaded) for a) terrain removed and b) terrain doubled, deformation frontogenesis [K (100 $\mathrm{km} \mathrm{h}^{-1}$; shaded; positive red] for c) terrain removed and d) terrain doubled, and diabatic frontogenesis [ $\mathrm{K}\left(100 \mathrm{~km} \mathrm{~h}^{-1}\right.$; shaded; positive red] for e) terrain removed and $\mathrm{f}$ ) terrain doubled.

Figure 4.30. Total accumulated precipitation ( $\mathrm{mm}$ ) from WRF domain 2 for the simulation with a) no evaporation from precipitation, b) no latent heating, and c) no surface heat fluxes.

Figure 4.31. WRF domain 1 300-hPa height (gpm; thick contoured), wind ( $\mathrm{m} \mathrm{s}^{-1}$; shaded; thin streamlines), and divergence $\left(10^{-5} \mathrm{~s}^{-1}\right.$; contoured; positive red) for the simulation with no evaporation at a) 1200 UTC 03 October 2015 and b) 1200 UTC 04 October 2015, with no latent heating at c) 1200 UTC 03 October 2015 and d) 1200 UTC 04 October 2015, and with no surface heat fluxes at

e) 1200 UTC 03 October 2015 and f) 1200 UTC 04 October 2015

Figure 4.32. WRF results for domain 1 at 1200 UTC 03 October 2015 displaying precipitable water ( $\mathrm{mm}$; shaded), sea level pressure ( $\mathrm{hPa}$; contoured), and 850-hPa wind $\left(\mathrm{m} \mathrm{s}^{-1}\right)$ for the simulation with a) no evaporation, b) no latent heating, and c) no surface heat fluxes.

Figure 4.33. WRF results for domain 2 at 1200 UTC 03 October 2015 displaying sea level pressure (hPa; contoured), 2-m temperature $\left({ }^{\circ} \mathrm{C}\right.$; shaded), and $10-\mathrm{m}$ wind $\left(\mathrm{m} \mathrm{s}^{-1}\right)$ for the simulation with a) no evaporation, b) no latent heating, and c) no surface heat fluxes. 
Figure 4.34. WRF domain 2 cross sections for the simulation with no evaporation at 1200 UTC 03 October 2015 for line segment CD (Fig. 4.8d) displaying potential temperature (K; contoured) and circulation vectors $\left(\mathrm{m} \mathrm{s}^{-1}, \mathrm{dPa} \mathrm{s}^{-1}\right)$ with a) potential vorticity (PVU; shaded) and b) divergence, c) deformation, d) tilting, and e) diabatic frontogenesis $\left[\mathrm{K}(100 \mathrm{~km} \mathrm{~h})^{-1}\right.$; shaded; positive red, negative blue], and f) $\mathrm{RH}$ (shaded) with $\theta_{e}(\mathrm{~K}$; contoured).

Figure 4.35. Precipitation (mm) for the 2011 flood event from 1200 UTC 06 September 2011 to 1200 UTC 09 September 2011 for domain 2 of the WRF control simulation a) at the location of maximum precipitation, plotted as accumulation with time and $b$ ) as the event total for the region. The transparent star represents the location of maximum precipitation. .98

Figure 4.36. WRF domain 1 300-hPa height (gpm; thick contoured), wind ( $\mathrm{m} \mathrm{s}^{-1}$; shaded; thin streamlines), and divergence $\left(10^{-5} \mathrm{~s}^{-1}\right.$; contoured; positive red) at a) 0300 UTC 08 September 2011 and b) 0000 UTC 09 September 2011 of the control simulation.

Figure 4.37. WRF results for domain 1 of the control simulation at 0300 UTC 08 September 2011 displaying a) precipitable water (mm; shaded), sea level pressure ( $\mathrm{hPa}$; contoured), 850-hPa wind $\left(\mathrm{m} \mathrm{s}^{-1}\right)$, and b) integrated water vapor transport (IVT, $\mathrm{kg} \mathrm{m}^{-1} \mathrm{~s}^{-1}$ ).

Figure 4.38. WRF results for domain 2 of the control simulation at 0300 UTC 08 September 2011 displaying 10-m wind $\left(\mathrm{m} \mathrm{s}^{-1}\right)$, a) sea level pressure (hPa; contoured), 2-m temperature $\left({ }^{\circ} \mathrm{C}\right.$; shaded), line segment KL, b) 2-m temperature $\left({ }^{\circ} \mathrm{C}\right.$; dashed), and $950-\mathrm{hPa}$ reflectivity ( $\mathrm{dBZ}$; shaded).

Figure 4.39. WRF domain 2 cross section from the control simulation at 0000 UTC 08 September 2011 for line segment KL (Fig. 4.38a) displaying potential vorticity (PVU; shaded red), potential temperature (K; contoured), and circulation vectors $\left[\mathrm{m} \mathrm{s}^{-1}, \mathrm{dPa} \mathrm{s}^{-1}\left(1 \mathrm{dPa} \mathrm{s}^{-1}=10^{-3} \mathrm{mb} \mathrm{s}^{-1}\right)\right]$.

Figure 4.40. Cross sections KL (Fig. 4.38a) showing a) tilting, b) deformation, c) divergence, and d) diabatic frontogenesis $\left[\mathrm{K}(100 \mathrm{~km} \mathrm{~h})^{-1}\right.$; shaded; positive red, negative blue], potential temperature $(\mathrm{K}$; contoured), and circulation vectors $\left[\mathrm{m} \mathrm{s}^{-1}, \mathrm{dPa} \mathrm{s}^{-1}\left(1 \mathrm{dPa} \mathrm{s}^{-1}=10^{-3} \mathrm{mb} \mathrm{s}^{-1}\right)\right]$ at 0000 UTC 08 September 2011 from WRF domain 2 of the control simulation.

Figure 4.41. Cross sections KL (Fig. 4.38a) showing equivalent potential temperature (K; contoured), a) relative humidity (shaded), b) equivalent potential vorticity (PVU; shaded), and circulation vectors $\left[\mathrm{m} \mathrm{s}^{-1}, \mathrm{dPa} \mathrm{s}^{-1}\left(1 \mathrm{dPa} \mathrm{s}^{-1}=10^{-3} \mathrm{mb} \mathrm{s}^{-}\right.\right.$ $\left.{ }^{1}\right)$ ] at 0000 UTC 08 September 2011 from WRF domain 2 of the control simulation. 
Figure 4.42. Parcel trajectories for the control simulation of the 2011 flood event beginning at 0600 UTC 08 September 2011 in the region of heavy rainfall, point $\mathrm{K}$, and working backward six hours. The trajectories are colored by parcel pressure at point $\mathrm{K}$. The arrows on the trajectory represent the height of the parcel at that time, with larger arrows representing a higher level...106

Figure 4.43. WRF sounding from domain 2 of the control simulation at point K (Fig. 4.42) showing temperature $\left({ }^{\circ} \mathrm{C}\right.$; red), dew point $\left({ }^{\circ} \mathrm{C}\right.$; blue $)$, and wind $\left(\mathrm{m} \mathrm{s}^{-1}\right)$ at 0600 UTC 08 September 2011.

Figure 4.44. WRF results of the simulation with the tropical cyclone removed displaying a) total accumulated precipitation $(\mathrm{mm})$ for domain 2 and b) precipitable water (mm; shaded), sea level pressure (hPa; contoured), and 850-hPa wind $\left(\mathrm{m} \mathrm{s}^{-1}\right)$ for domain 1 at 0300 UTC 08 September 2011

Figure 4.45. WRF results of the simulation with terrain height doubled displaying a) total accumulated precipitation $(\mathrm{mm})$ for domain 2 and b) precipitable water ( $\mathrm{mm}$; shaded), sea level pressure (hPa; contoured), and 850-hPa wind $\left(\mathrm{m} \mathrm{s}^{-1}\right)$ for domain 1 at 0300 UTC 08 September 2011.

Figure 4.46. WRF results of the simulation with terrain removed displaying a) total accumulated precipitation $(\mathrm{mm})$ for domain 2 and $\mathrm{b}$ ) precipitable water $(\mathrm{mm}$; shaded), sea level pressure (hPa; contoured), and 850-hPa wind $\left(\mathrm{m} \mathrm{s}^{-1}\right)$ for domain 1 at 0300 UTC 08 September 2011.

Figure 4.47. WRF results for domain 2 of the simulation with terrain removed at 0300 UTC 08 September 2011 displaying sea level pressure (hPa; contoured), 10$\mathrm{m}$ wind $\left(\mathrm{m} \mathrm{s}^{-1}\right)$, and $2-\mathrm{m}$ temperature $\left({ }^{\circ} \mathrm{C}\right.$; shaded $)$.

Figure 4.48. Total accumulated precipitation ( $\mathrm{mm}$ ) from WRF domain 2 for the simulation with a) no evaporation from precipitation, b) no latent heating, and c) no surface heat fluxes.

Figure 4.49. WRF results for the simulation with no latent heating at 0300 UTC 08 September 2011 displaying a) precipitable water (mm; shaded), sea level pressure (hPa; contoured), and 850-hPa wind $\left(\mathrm{m} \mathrm{s}^{-1}\right)$ from domain 1 and b) sea level pressure ( $\mathrm{hPa}$; contoured), 10-m wind $\left(\mathrm{m} \mathrm{s}^{-1}\right)$, and 2-m temperature $\left({ }^{\circ} \mathrm{C}\right.$; shaded) from domain 2 .

Figure 4.50. WRF results for the simulation with no surface heat fluxes at 0300 UTC 08 September 2011 displaying a) precipitable water (mm; shaded), sea level pressure (hPa; contoured), and 850-hPa wind $\left(\mathrm{m} \mathrm{s}^{-1}\right)$ from domain 1 and b) sea level pressure ( $\mathrm{hPa}$; contoured), 10-m wind $\left(\mathrm{m} \mathrm{s}^{-1}\right)$, and 2-m temperature $\left({ }^{\circ} \mathrm{C}\right.$; shaded $)$ from domain 2 . 
Figure 4.51. Precipitation (mm) for the 2016 event from 1200 UTC 28 September 2016 to 0000 UTC 01 October 2016 for domain 2 of the WRF control simulation a) at the location of maximum precipitation in North Carolina, plotted as accumulation with time and $b$ ) as the event total for the region. The transparent star represents the location of maximum precipitation.

Figure 4.52. WRF domain 1 300-hPa height (gpm; thick contoured), wind ( $\mathrm{m} \mathrm{s}^{-1}$; shaded; thin streamlines), and divergence $\left(10^{-5} \mathrm{~s}^{-1}\right.$; contoured; positive red) at a) 1200 UTC 29 September 2016 and b) 1200 UTC 30 September 2016 of the control simulation.

Figure 4.53. WRF results for domain 1 of the control simulation at 0000 UTC 30 September 2016 displaying a) precipitable water (mm; shaded), sea level pressure ( $\mathrm{hPa}$; contoured), 850-hPa wind $\left(\mathrm{m} \mathrm{s}^{-1}\right)$, and b) integrated water vapor transport (IVT; $\mathrm{kg} \mathrm{m}^{-1} \mathrm{~s}^{-1}$ ).

Figure 4.54. WRF results for domain 2 of the control simulation at 1500 UTC 29 September 2016 displaying 10-m wind $\left(\mathrm{m} \mathrm{s}^{-1}\right)$, a) sea level pressure (hPa; contoured), 2-m temperature $\left({ }^{\circ} \mathrm{C}\right.$; shaded), line segment $\left.\mathrm{MN}, \mathrm{b}\right) 2-\mathrm{m}$ temperature $\left({ }^{\circ} \mathrm{C}\right.$; dashed), and 950-hPa reflectivity (dBZ; shaded).

Figure 4.55. WRF domain 2 cross section from the control simulation at 1800 UTC 29 September 2016 for line segment MN (Fig. 4.53a) displaying potential vorticity (PVU; shaded red), potential temperature (K; contoured), and circulation vectors $\left[\mathrm{m} \mathrm{s}^{-1}, \mathrm{dPa} \mathrm{s}^{-1}\left(1 \mathrm{dPa} \mathrm{s}^{-1}=10^{-3} \mathrm{mb} \mathrm{s}^{-1}\right)\right]$.

Figure 4.56. Cross sections MN (Fig. 4.54a) showing a) tilting, b) deformation, c) divergence, and d) diabatic frontogenesis [K (100 km h) ${ }^{-1}$; shaded; positive red, negative blue], potential temperature ( $\mathrm{K}$; contoured), and circulation vectors $\left[\mathrm{m} \mathrm{s}^{-1}, \mathrm{dPa} \mathrm{s}^{-1}\left(1 \mathrm{dPa} \mathrm{s}^{-1}=10^{-3} \mathrm{mb} \mathrm{s}^{-1}\right)\right]$ at 1800 UTC 29 September 2016 from WRF domain 2 of the control simulation.

Figure 4.57. Cross sections MN (Fig. 4.54a) showing equivalent potential temperature ( $\mathrm{K}$; contoured), a) relative humidity (shaded), b) equivalent potential vorticity (PVU; shaded), and circulation vectors $\left[\mathrm{m} \mathrm{s}^{-1}, \mathrm{dPa} \mathrm{s}^{-1}\left(1 \mathrm{dPa} \mathrm{s}^{-1}=\right.\right.$ $\left.10^{-3} \mathrm{mb} \mathrm{s}^{-1}\right)$ ] at 1800 UTC 29 September 2016 from WRF domain 2 of the control simulation.

Figure 4.58. WRF domain 2 sounding from the control simulation at 1800 UTC 29 September 2016 for the location of maximum precipitation showing temperature $\left({ }^{\circ} \mathrm{C}\right.$; red $)$, dew point $\left({ }^{\circ} \mathrm{C}\right.$; blue $)$, and wind $\left(\mathrm{m} \mathrm{s}^{-1}\right)$.

Figure 4.59. WRF results of the simulation with terrain removed displaying a) total accumulated precipitation $(\mathrm{mm})$ for domain 2 and b) precipitable water $(\mathrm{mm}$; shaded), sea level pressure (hPa; contoured), and $850-\mathrm{hPa}$ wind $\left(\mathrm{m} \mathrm{s}^{-1}\right)$ for domain 1 at 0000 UTC 30 September 2016. 125 
Figure 4.60. WRF results for domain 2 of the simulation with no terrain at 1500 UTC 29 September 2016 displaying 10-m wind $\left(\mathrm{m} \mathrm{s}^{-1}\right)$, a) sea level pressure ( $\mathrm{hPa}$; contoured), 2-m temperature $\left({ }^{\circ} \mathrm{C}\right.$; shaded $)$, b) 2-m temperature $\left({ }^{\circ} \mathrm{C}\right.$; dashed), and 950-hPa reflectivity (dBZ; shaded).

Figure 4.61. Total accumulated precipitation ( $\mathrm{mm}$ ) from WRF domain 2 for the simulation with a) no evaporation from precipitation, b) no latent heating, and c) no surface heat fluxes.

Figure 4.62. Precipitation (mm) for the 2014 event from 1200 UTC 12 August 2014 to 0600 UTC 14 August 2014 for domain 2 of the WRF control simulation a) at the location of maximum precipitation, displaced southeast of Long Island, New York, plotted as accumulation with time and b) as the event total for the region. The transparent star represents the location of maximum precipitation.

Figure 4.63. WRF control simulation results at 1800 UTC 13 August 2014 300-hPa height (gpm; thick contoured), wind ( $\mathrm{m} \mathrm{s}^{-1}$; shaded; thin streamlines), and divergence $\left(10^{-5} \mathrm{~s}^{-1}\right.$; contoured; positive red) for a) domain 1 and $\left.\mathrm{b}\right)$ domain 2 of the control simulation. 130

Figure 4.64. WRF results for domain 1 of the control simulation at 1800 UTC 13 August 2014 displaying a) precipitable water ( $\mathrm{mm}$; shaded), sea level pressure (hPa; contoured), 850-hPa wind ( $\left.\mathrm{m} \mathrm{s}^{-1}\right)$, and b) integrated water vapor transport (IVT; $\left.\mathrm{kg} \mathrm{m}^{-1} \mathrm{~s}^{-1}\right)$.

Figure 4.65. WRF results for domain 2 of the control simulation at 1800 UTC 13 August 2014 displaying 10-m wind $\left(\mathrm{m} \mathrm{s}^{-1}\right)$, a) sea level pressure ( $\mathrm{hPa}$; contoured), 2m temperature $\left({ }^{\circ} \mathrm{C}\right.$; shaded $)$, line segment $\left.\mathrm{OP}, \mathrm{b}\right) 2$-m temperature $\left({ }^{\circ} \mathrm{C}\right.$; dashed), and 950-hPa reflectivity (dBZ; shaded).

Figure 4.66. WRF domain 2 cross section from the control simulation at 1800 UTC 13 August 2014 for line segment OP (Fig. 4.63a) displaying potential vorticity (PVU; shaded red), potential temperature ( $\mathrm{K}$; contoured), and circulation vectors $\left[\mathrm{m} \mathrm{s}^{-1}, \mathrm{dPa} \mathrm{s}^{-1}\left(1 \mathrm{dPa} \mathrm{s}^{-1}=10^{-3} \mathrm{mb} \mathrm{s}^{-1}\right)\right]$.

Figure 4.67. Cross sections OP (Fig. 4.65a) showing a) tilting, b) deformation, c) divergence, and d) diabatic frontogenesis $\left[\mathrm{K}(100 \mathrm{~km} \mathrm{~h})^{-1}\right.$; shaded; positive red, negative blue], potential temperature $(\mathrm{K}$; contoured), and circulation vectors $\left[\mathrm{m} \mathrm{s}^{-1}, \mathrm{dPa} \mathrm{s}^{-1}\left(1 \mathrm{dPa} \mathrm{s}^{-1}=10^{-3} \mathrm{mb} \mathrm{s}^{-1}\right)\right]$ at $1800 \mathrm{UTC} 13$ August 2014 from WRF domain 2 of the control simulation.

Figure 4.68. Cross sections OP (Fig. 4.65a) showing equivalent potential temperature (K; contoured), a) relative humidity (shaded), b) equivalent potential vorticity (PVU; shaded), and circulation vectors $\left[\mathrm{m} \mathrm{s}^{-1}, \mathrm{dPa} \mathrm{s}^{-1}\left(1 \mathrm{dPa} \mathrm{s}^{-1}=10^{-3} \mathrm{mb} \mathrm{s}^{-}\right.\right.$ $\left.{ }^{1}\right)$ ] at 1800 UTC 13 August 2014 from WRF domain 2 of the control simulation. 
Figure 4.69. WRF sounding from domain 2 of the control simulation at 1800 UTC 13 August 2014 for the location of maximum precipitation showing temperature $\left({ }^{\circ} \mathrm{C}\right.$; red $)$, dew point $\left({ }^{\circ} \mathrm{C}\right.$; blue $)$, and wind $\left(\mathrm{m} \mathrm{s}^{-1}\right)$.

Figure 4.70. WRF results from domain 2 of the simulation with terrain removed displaying a) total accumulated precipitation $(\mathrm{mm})$ and $\mathrm{b}$ ) sea level pressure (hPa; contoured), 2-m temperature $\left({ }^{\circ} \mathrm{C}\right.$; shaded), and $10-\mathrm{m}$ wind $\left(\mathrm{m} \mathrm{s}^{-1}\right)$ at 1800 UTC 13 August 2014.

Figure 4.71. Total accumulated precipitation ( $\mathrm{mm}$ ) from WRF domain 2 for the simulation with a) no evaporation from precipitation, b) no latent heating, and c) no surface heat fluxes.

Figure 4.72. Precipitation (mm) for the 2010 event from 0000 UTC 29 September 2010 to 1800 UTC 01 October 2010 for domain 2 of the WRF control simulation a) at the location of maximum precipitation, off the coast of Myrtle Beach, South Carolina, plotted as accumulation with time and b) as the event total for the region. The transparent star represents the location of maximum precipitation.

Figure 4.73. WRF domain $1300-\mathrm{hPa}$ height (gpm; thick contoured), wind ( $\mathrm{m} \mathrm{s}^{-1}$; shaded; thin streamlines), and divergence $\left(10^{-5} \mathrm{~s}^{-1}\right.$; contoured; positive red) at a) 1200 UTC 29 September 2010 and b) 0000 UTC 01 October 2010 of the control simulation.

Figure 4.74. WRF results for domain 1 of the control simulation at 1800 UTC 30 September 2010 displaying a) precipitable water (mm; shaded), sea level pressure ( $\mathrm{hPa}$; contoured), $850-\mathrm{hPa}$ wind $\left(\mathrm{m} \mathrm{s}^{-1}\right)$, and b) integrated water vapor transport (IVT; $\mathrm{kg} \mathrm{m}^{-1} \mathrm{~s}^{-1}$ ).

Figure 4.75. WRF results for domain 2 of the control simulation at 1800 UTC 30 September 2010 displaying 10-m wind $\left(\mathrm{m} \mathrm{s}^{-1}\right)$, a) sea level pressure (hPa; contoured), 2-m temperature $\left({ }^{\circ} \mathrm{C}\right.$; shaded), line segment $\left.\mathrm{QR}, \mathrm{b}\right) 2-\mathrm{m}$ temperature $\left({ }^{\circ} \mathrm{C}\right.$; dashed), and $950-\mathrm{hPa}$ reflectivity (dBZ; shaded).

Figure 4.76. WRF domain 2 cross section from the control simulation at 1800 UTC 30 September 2010 for line segment QR (Fig. 4.75a) displaying potential vorticity (PVU; shaded red), potential temperature (K; contoured), and circulation vectors $\left[\mathrm{m} \mathrm{s}^{-1}, \mathrm{dPa} \mathrm{s}^{-1}\left(1 \mathrm{dPa} \mathrm{s}^{-1}=10^{-3} \mathrm{mb} \mathrm{s}^{-1}\right)\right]$.

Figure 4.77. Cross sections QR (Fig. 4.75a) showing a) tilting, b) deformation, c) divergence, and d) diabatic frontogenesis [K $\left(100 \mathrm{~km} \mathrm{~h}^{-1}\right.$; shaded; positive red, negative blue], potential temperature $(\mathrm{K}$; contoured), and circulation vectors $\left[\mathrm{m} \mathrm{s}^{-1}, \mathrm{dPa} \mathrm{s}^{-1}\left(1 \mathrm{dPa} \mathrm{s}^{-1}=10^{-3} \mathrm{mb} \mathrm{s}^{-1}\right)\right]$ at 1800 UTC 30 September 2010 from WRF domain 2 of the control simulation. 
Figure 4.78. Cross sections QR (Fig. 4.75a) showing equivalent potential temperature (K; contoured), a) relative humidity (shaded), b) equivalent potential vorticity (PVU; shaded), and circulation vectors $\left[\mathrm{m} \mathrm{s}^{-1}, \mathrm{dPa} \mathrm{s}^{-1}\left(1 \mathrm{dPa} \mathrm{s}^{-1}=10^{-3} \mathrm{mb} \mathrm{s}^{-}\right.\right.$ $\left.{ }^{1}\right)$ ] at 1800 UTC 30 September 2010 from WRF domain 2 of the control simulation.

Figure 4.79. WRF domain 2 sounding from the control simulation at 1800 UTC 30 September 2010 for the location of maximum precipitation showing temperature $\left({ }^{\circ} \mathrm{C}\right.$; red), dew point $\left({ }^{\circ} \mathrm{C}\right.$; blue $)$, and wind $\left(\mathrm{m} \mathrm{s}^{-1}\right)$.

Figure 4.80. WRF results of the simulation with terrain removed displaying a) total accumulated precipitation $(\mathrm{mm})$ for domain 2 and $\mathrm{b}$ ) precipitable water ( $\mathrm{mm}$; shaded), sea level pressure (hPa; contoured), and 850-hPa wind $\left(\mathrm{m} \mathrm{s}^{-1}\right)$ for domain 1 at 1800 UTC 30 September 2010.

Figure 4.81. WRF results for domain 2 of the simulation with terrain removed at 1800 UTC 30 September 2010 displaying 10-m wind $\left(\mathrm{m} \mathrm{s}^{-1}\right)$, a) sea level pressure (hPa; contoured), 2-m temperature $\left({ }^{\circ} \mathrm{C}\right.$; shaded), b) 2-m temperature $\left({ }^{\circ} \mathrm{C}\right.$; dashed), and 950-hPa reflectivity (dBZ; shaded).

Figure 4.82. Total accumulated precipitation $(\mathrm{mm})$ from WRF domain 2 for the simulation with a) no evaporation from precipitation and b) no surface heat fluxes.

Figure 4.83. WRF domain 1 results of the simulation with no latent heating at 1800 UTC 30 September 2010 displaying sea level pressure (hPa; contoured), a) precipitable water (mm; shaded), 850-hPa wind $\left.\left(\mathrm{m} \mathrm{s}^{-1}\right), \mathrm{b}\right) 2$-m temperature $\left({ }^{\circ} \mathrm{C}\right.$; shaded $)$, and $10-\mathrm{m}$ wind $\left(\mathrm{m} \mathrm{s}^{-1}\right)$.

Figure 4.84. WRF results for domain 1 of the simulation with no surface heat fluxes at 1800 UTC 30 September 2010 displaying sea level pressure $(\mathrm{hPa}$; contoured), a) precipitable water (mm; shaded), 850-hPa wind $\left(\mathrm{m} \mathrm{s}^{-1}\right)$, b) 2-m temperature $\left({ }^{\circ} \mathrm{C}\right.$; shaded $)$, and $10-\mathrm{m}$ wind $\left(\mathrm{m} \mathrm{s}^{-1}\right)$.

Figure A.1. WRF model domains representing the outer domain (domain 1) of 15-km grid spacing with the inner domain (d02) of 3-km grid spacing outlined in blue for use in simulating the heavy precipitation in a) central Pennsylvania and New York in September 2011, b) North Carolina in September 2016, c) Islip, New York in August 2014, and d) North Carolina in September 2010. 
Figure A.2. Precipitation amount for the simulation of the Pennsylvania/New York heavy rain event from 1200 UTC 06 September 2011 to 1200 UTC 09 September 2011 created using a) 4-km NCEP Stage IV reanalysis data from Earth Observing Laboratory (EOL) and b) WRF control simulation output for domain 2. The MODE results are shown to identify an object (red) for c) the reanalysis data shown in a) and d) the WRF data shown in b) that are corresponding in both analyses based on a threshold of $254 \mathrm{~mm}$. The total interest value calculated in MODE for the objects (red) shown in c) and d) is 0.998 164

Figure A.3. Hurricane Katia a) tracks and b) central pressures from 1200 UTC 06 September 2011 to 1200 UTC 09 September 2011 resulting from the National Hurricane Center best track analysis (black), WRF d01 control simulation (red), and the experimental WRF d01 simulations with terrain removed (blue) and terrain doubled (green). .165

Figure A.4. Precipitation amount for the simulation of the North Carolina rain event from 1200 UTC 28 September 2016 to 0000 UTC 01 October 2016 created using a) 4-km NCEP Stage IV reanalysis data from Earth Observing Laboratory (EOL) and b) WRF control simulation output for domain 2. The MODE results are shown to identify an object (red) for c) the reanalysis data shown in a) and d) the WRF data shown in $b$ ) that are corresponding in both analyses based on a threshold of $254 \mathrm{~mm}$. The total interest value calculated in MODE for the objects (red) shown in c) and d) is 0.91 .

Figure A.5. Precipitation amount for the simulation of the Islip, New York heavy rain event from 1200 UTC 12 August 2014 to 0600 UTC 14 August 2014 created using a) 4-km NCEP Stage IV reanalysis data from Earth Observing Laboratory (EOL) and b) WRF control simulation output for domain 2. The MODE results are shown to identify an object (red) for c) the reanalysis data shown in a) and d) the WRF data shown in b) that are corresponding in both analyses based on a threshold of $127 \mathrm{~mm}$. The total interest value calculated in MODE for the objects (red) shown in c) and d) is 0.85 .

Figure A.6. Precipitation amount for the simulation of the North Carolina heavy rain event from 0000 UTC 29 September 2010 to 1800 UTC 01 October 2010 created using a) 4-km NCEP Stage IV reanalysis data from Earth Observing Laboratory (EOL) and b) WRF control simulation output for domain 2. The MODE results are shown to identify an object (red) for c) the reanalysis data shown in a) and d) the WRF data shown in b) that are corresponding in both analyses based on a threshold of $254 \mathrm{~mm}$. The total interest value calculated in MODE for the objects (red) shown in c) and d) is 0.94 .168 
Figure B.1. Climate Forecast System Reanalysis (CFSR) valid at 0600 UTC 08 September 2011 representing 250-hPa wind ( $\mathrm{m} \mathrm{s}^{-1}$; shaded), 600-400-hPa ascent ( $5 \times 10^{-3} \mathrm{hPa} \mathrm{s}^{-1}$; dashed red), 300-200-hPa potential vorticity (PVU;

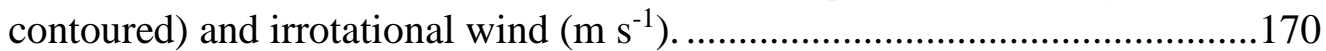

Figure B.2. As in Fig. B.1, except valid at 1800 UTC 29 September 2016. .................171

Figure B.3. As in Fig. B.1, except valid at 0600 UTC 13 August 2014 ........................172

Figure B.4. As in Fig. B.1, except valid at 1800 UTC 30 September 2010.................173 


\section{LIST OF TABLES}

Table 3.1. The parameterization schemes, and corresponding references, that created the most realistic control simulation of the South Carolina flood event of October 2015. These schemes were consistent throughout this research......................38 


\begin{abstract}
Coastal fronts are commonly found along the East Coast of the United States and can often be associated with intense rainfall and flooding due to elevated convection on

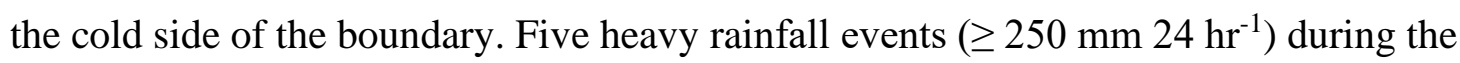
fall months along the East Coast were investigated using numerical weather prediction (NWP) models to determine the influence of an upper-level trough/cut-off low, an offshore tropical cyclone, a frontal boundary, and a moisture plume on the intense precipitation. Using experimental NWP simulations, it was determined that the tropical cyclone had an impact on the moisture plume and subsequent location of precipitation due to an associated deformation zone. The tropical cyclone prolonged the events by 6 hours, but inhibited the amount of moisture and resulting precipitation by deterring southeasterly flow. Evaporation from precipitation (surface heat fluxes) contributed to less than $25 \%(33 \%)$ of the precipitation, while latent heat release had the largest impact on the rain totals due to positive feedback from convection and an influence on the frontal boundary. Terrain also impacted the frontal boundary in each event, altering precipitation totals. Parcel trajectories confirmed regions of frontogenesis to be the main source of lift for the release of gravitational instability and convective initiation in each event, while the extratropical cyclone provided upper-level support for ascent and organized the plume of deep tropospheric moisture perpendicular to the front. Three of the five events lasted multiple days due to negative PV advection by the irrotational wind, in response to latent heat release in the region of convection, acting to slow the propagation of the upper-level low.
\end{abstract}




\section{CHAPTER 1. INTRODUCTION}

Receiving more than $250 \mathrm{~mm}$ ( 10 inches) of rainfall in a 24 -hour period is a great threat to life and property, especially in coastal areas of the eastern United States. It is common to expect this rainfall intensity with a landfalling hurricane on the East Coast. Preliminary research discovered landfalling hurricanes to be the cause of roughly 20 percent of precipitation events of the aforementioned magnitude in states along the East Coast (excluding Florida) in a 12-year period from 2005 to 2017. However, 33 percent of the intense precipitation events were caused by convection occurring on the cold side of a frontal boundary, likely elevated in nature. An event with $250 \mathrm{~mm}$ of rainfall in 24 hours is more likely to occur from elevated convection in coastal states than from a landfalling hurricane. While landfalling hurricanes may have a much greater impact on society (e.g., larger area of intense rainfall, strong winds, storm surge, etc.), they also come with much earlier warnings than that of elevated convection.

Elevated convection events (Means 1952; Corfidi et al. 2006) are typically only researched for the Central Plains of the United States. While climatologically the majority of elevated thunderstorms occur in the Central Plains (Colman 1990), their occurrence in other regions of the United States should not be overlooked due to their potential to cause heavy precipitation and flash flooding (Rochette and Moore 1996). The East Coast, in particular, is susceptible to elevated convection due to the location and orientation of the Appalachian Mountains. Stable surface layers between this mountain range and the coast are common due to cold-air damming or the presence of a coastal 
front. With added synoptic forcing, the region can experience elevated convection and heavy precipitation.

Along the East Coast, two record-breaking rainfall events associated with elevated convection occurred in September 2011 and October 2015, associated with a well-offshore tropical cyclone. The synoptic setup for both events involved the tropical cyclone in the Atlantic, as well as an extratropical cyclone located over the eastern United States. The deformation zone between the cyclones in both events created significant onshore flow of moisture that interacted with a stalled frontal boundary and resulted in elevated convection, with rainfall amounts totaling over $250 \mathrm{~mm}$ in 24 hours. Life-threatening flooding and extensive damage occurred in coastal states with each event. The October 2015 flood alone was responsible for 19 fatalities, nearly 1.5 billion dollars in damage, over 50 dam failures, the closure of 410 roads and bridges, and the loss of power to 50,000 residents in the state of South Carolina (FEMA 2016; Murphy 2016). The September 2011 event led to 200 million dollars in damage and 10 fatalities in Pennsylvania, with nearly one billion dollars of damage in New York (Brown 2012). In either case, the major tropical cyclone never even made landfall. Initial analysis of each event suggests the greatest contributor to the rainfall was the stagnant coastal front, which contributed to the intensity of the elevated convection, while the cyclone positions and deformation zones strongly influenced the location of the precipitation.

With the hypothesis of coastal fronts being the main contributor to elevated convection along the East Coast, an examination of three additional East Coast heavy precipitation events involving a frontal boundary that were not influenced by an offshore tropical cyclone was necessary to identify and compare the processes involved in 
initiating convection for each case. Each of the three additional events resulted in over $250 \mathrm{~mm}$ of precipitation in coastal states and occurred during the late summer or early fall. These high amounts of rainfall over short periods of time led to flash flooding, loss of life, and damage to property. In order to provide advanced warnings, it is important to diagnose the cause of these events and be able to predict the onset and location of convection as early as possible.

\subsection{Objectives}

With the exact cause of elevated convection along the East Coast in question, this study defines the extent to which cyclones and coastal fronts contribute to heavy precipitation and flooding events. Five cases of heavy precipitation associated with coastal fronts and extratropical cyclones along the East Coast were investigated, with the South Carolina flood of October 2015 completed as the pilot study due to the significant societal impact. Various techniques on several scales of motion were used to analyze each event. The specific objectives of this research were as follows:

1. Investigate the sources of moisture and causes of lower-tropospheric convergence prior to heavy rainfall events ( $\geq 250 \mathrm{~mm}$ in 24 hours) along the East Coast

2. Determine the role of each synoptic or mesoscale feature, as well as the underlying terrain, on the coastal front and subsequent convection during the event 
3. Assess the mechanisms responsible for convective initiation and maintenance; verify the type of convection, elevated or surface-based. 


\section{CHAPTER 2. MOTIVATION}

\subsection{Literature Review}

The United States Atlantic Coast is no stranger to intense precipitation associated with landfalling tropical cyclones, such as Hurricane Agnes (1972), Hurricane Floyd (1999), and Hurricane Matthew (2016). However, tropical cyclones can also contribute to heavy rainfall, without making landfall, through large-scale interactions with other cyclones or frontal boundaries. It is well known that tropical cyclones can interact with, and even influence, mid-latitude atmospheric features, such as upper-tropospheric troughs or frontal boundaries. Trough interaction can lead to strength changes in the tropical cyclone (Lewis and Jorgensen 1978; Hanley et al. 2001), extratropical transition of the cyclone (Peng et al. 2007), or even a deformed trough due to the tropical cyclone flow (Kimball and Evans 2002). It is important to not only understand these interactions, but also to understand how these interactions impact society through precipitation.

Tropical cyclones can change the flow of a trough and can also impact the movement of a frontal boundary (Ross and Kurihara 1995). A change in the movement of a front means a change in the precipitation associated with the front, which has a huge societal impact. If a tropical cyclone slows the motion of a front, a region can receive large amounts of precipitation, potentially resulting in flooding. Alternatively, when a front moves more quickly, less precipitation may fall in a region. For example, Hurricane Gloria (1985) caused the acceleration of a cold front over the eastern United States due to an increase in westerly winds south of the hurricane, resulting in a reduction of 
accumulated frontal precipitation southwest of the hurricane (Ross and Kurihara 1995). Frontal boundaries may also influence precipitation distribution during landfalling tropical cyclones, as was the case with Hurricane Ernesto (2006; Dale and Lackmann 2014).

Large-scale interactions involving tropical cyclones and moisture transport have previously been studied. Typhoon Songda (2004) was associated with a northward moisture transport that contributed to heavy rainfall in Japan, impacts were exacerbated because the moisture was transported into a region preconditioned for precipitation (Wang et al. 2009). This type of precipitation event, when it occurs in the United States, has been termed a predecessor rain event (PRE) by Cote (2007); a mesoscale rainfall event occurring approximately $1000 \mathrm{~km}$ poleward of a tropical cyclone with rainfall rates exceeding $100 \mathrm{~mm}$ in 24 hours. To be considered a PRE, an event must also involve the poleward advection of deep tropical moisture, originating from the tropical cyclone, into the rainfall region (Galarneau et al. 2010). Hurricane Joaquin (2015) was associated with a poleward advected column of tropical moisture that may have contributed to flooding in South Carolina (Marciano and Lackmann 2017). A similar setup occurred with Hurricane Katia (2011; Jurewicz 2012; Gitro et al. 2014).

Hurricane Katia (2011) and Hurricane Joaquin (2015) were involved in a rare interaction between an upper-tropospheric trough and an associated cut-off low, as well as a stalled coastal front, leading to a deformation zone with significant moisture transport; this caused elevated convection and heavy precipitation in coastal states even though the tropical cyclone did not make landfall. This research was aimed at increasing the limited knowledge on how the resultant flow of such an interaction can concentrate 
copious amounts of moisture into one region for a significant amount of time, the sources of the moisture incorporated into the flow, how the synoptic and mesoscale features impact the strength and movement of other features in the environment, how tropical cyclone positions can aid in predicting the location of heavy rainfall, why convection occurs as a result of these interactions, and why the convection was elevated in nature.

\subsection{Case Selection}

Three additional cases of heavy precipitation with possible elevated convection along the East Coast were identified, each involving upper-level lows and frontal boundaries with no tropical cyclone influence. Two of these events occurred in North Carolina in 2010 and 2016, and the third event occurred in Long Island, New York in 2014. This research expands on the knowledge of multiscale interactions and elevated convection along the East Coast through an intense investigation into all five cases of extreme precipitation and flooding. A new approach to the examination of a frontal boundary and ensuing elevated convection was employed using parcel trajectories and diagnostics, which are commonly used to analyze atmospheric chemistry (Polissar et al. 2001) or moisture transport (Moore et al. 2012). Associated thermodynamic profiles were used to analyze the environmental changes along a parcel trajectory as the parcel approaches and passes over a front. All five cases were analyzed for commonalities with the goal of finding a way to forecast the location, timing, and amounts of precipitation more accurately. It is especially crucial to conduct research that can improve precipitation forecasts and increase the lead times of flood warnings, as flood events, which greatly 
threaten life and property, have been predicted to increase in the future due to a warming atmosphere (e.g., Trenberth et al. 2005; Knutson et al. 2010).

\subsection{East Coast Extreme Rain}

Following the historic rainfall over the coastal Carolinas of the United States in early October 2015, questions arose regarding the frequency of such events in that region. The October 2015 event was unique in that a significant tropical cyclone was present well off the U.S. East Coast, but with early media accounts attributing much of the rainfall to the cyclone itself. Ensuing work has shown that this was not the case (Henson and Market 2017; Marciano and Lackmann 2017), and that terrain and other mesoscale features played key roles in focusing the extreme rains in that event.

With this as motivation, and as a prelude to further investigations of extreme rainfall cases along the East Coast, a brief study was carried out on recent events to determine the frequency of tropical cyclones versus such phenomena as frontal boundaries (coastal and otherwise) and cold air damming. The Community Collaborative Rain, Hail and Snow (CoCoRaHS) Network was used to find dates of heavy rain $(\geq 250$ $\mathrm{mm} 24 \mathrm{hr}^{-1}$ ) for the East Coast from 2005 to 2017. Florida is excluded from the analysis, as a tropical environment requires a different methodology for classifying extreme rainfall events.

CoCoRaHS is a volunteer network of weather observers and was founded in 1998 to fill the need for "high-quality, high-density rainfall measurements" in the meteorological community (www.cocorahs.org). The organization began its nationwide 
expansion in 2005 and was eventually contributed to by observers in all 50 states in December 2009, with the number of registered observers growing from 2,000 to over 15,000 (Reges et al. 2016). This study of extreme rain for the East Coast begins in 2005 because CoCoRaHS contains reports of rain meeting the criteria for this study in 2005 for New York. From 2005 to 2009, the remaining 15 states involved in this study gradually produced rainfall observations for CoCoRaHS. Thus, it is important to note that this study is not an accurate representation of all possible extreme rainfall $(\geq 250 \mathrm{~mm} 24 \mathrm{hr}$ $\left.{ }^{1}\right)$ events along the East Coast for the years 2005 to 2017 nor is it a true climatology of such events.

For 16 states along the East Coast, 167 individual reports of rainfall totaling 250 $\mathrm{mm}$ or more in a 24-hour period were obtained by CoCoRaHS over the 12-year period. The individual reports make up 21 different events (Fig. 2.1). Events were classified by date, location, and associated atmospheric features that may have led to the significant precipitation. For example, there were 22 individual reports meeting the criteria from 0405 October 2015 in South Carolina. All 22 reports make up one event; the South Carolina flood involving a coastal front, an upper-level low, and Hurricane Joaquin (2015).

Of the 21 events of extreme precipitation, 4 (19\%) were caused by the landfall of a tropical cyclone. 100 individual reports were associated with the landfall of Hurricane Matthew on 08 and 09 October 2016 in the states of Georgia, South Carolina, North Carolina, and Virginia. On 03 September 2016, 5 reports of heavy rain in South Carolina were caused by the landfall of Hurricane Hermine. Hurricane Irene made landfall in late August 2011 and led to 15 reports of extreme rainfall over 4 different states; North Carolina, Virginia, Maryland, and New Jersey. In late August 2008, Tropical Storm Fay 


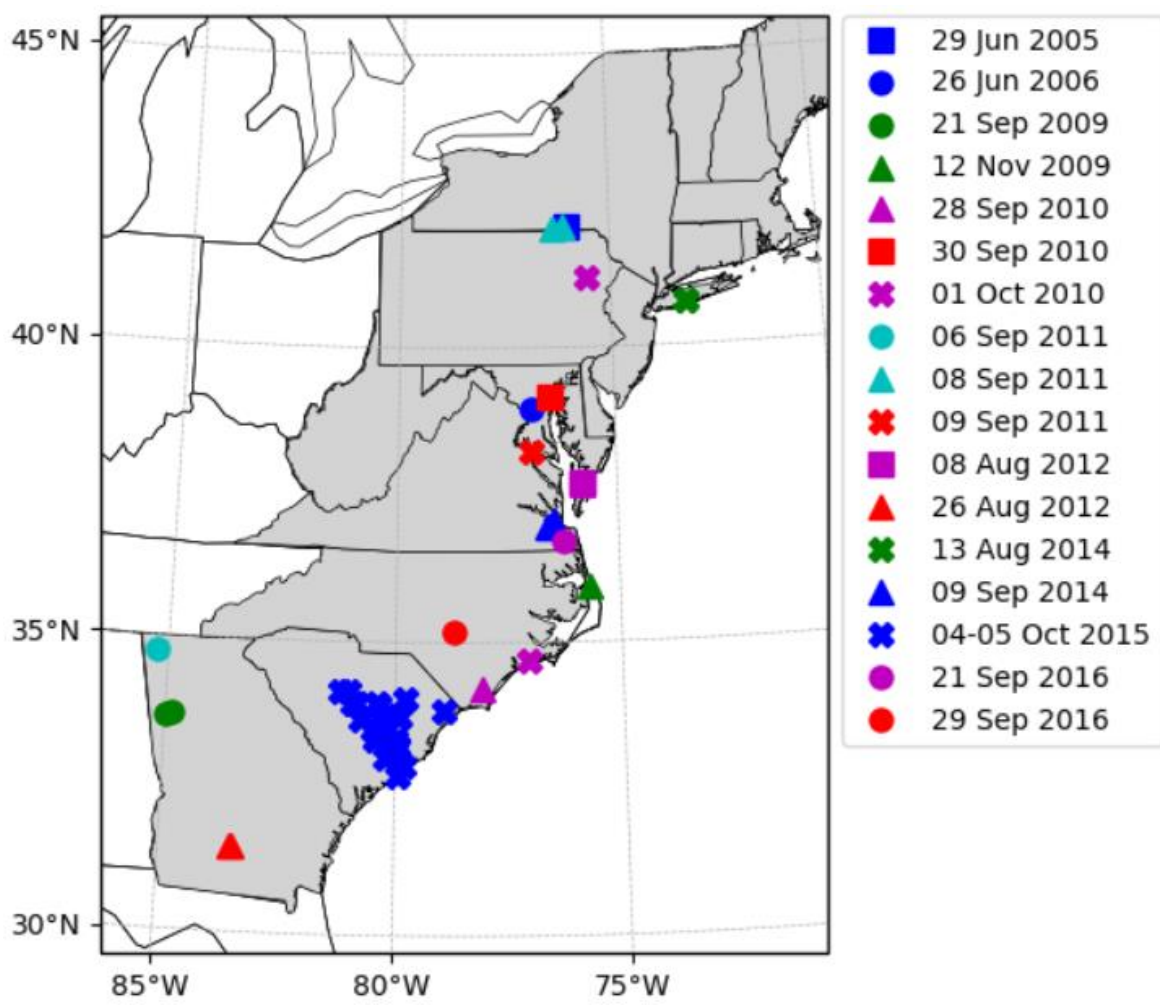

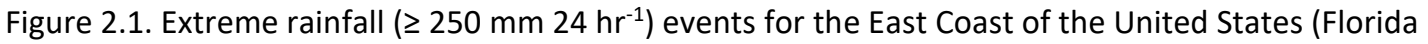
excluded) from 2005 to 2017, excluding events associated with the landfall of a tropical cyclone. Each individual report was obtained from the Community Collaborative Rain, Hail and Snow (CoCoRaHS) Network and is identified by the marking of its associated event.

located over northern Florida was the reason for 1 report of extreme rain in southern Georgia. Even though the number of individual reports and the area impacted by extreme rainfall is typically very large with landfalling tropical cyclones, the events of this classification make up a relatively small portion of all heavy rainfall events of this criterion along the East Coast.

The remaining 17 events (81\%) occurred near a frontal boundary. Of those 17 events involving a frontal boundary, 10 of the events ( $48 \%$ of all 21 events) and the associated reports fell on the warm side of the boundary. It is not uncommon to see 
extreme rain on the East Coast south and/or east of a frontal boundary due to plentiful warm, moist air from the Atlantic Ocean meeting colder, drier continental air. Heavy rain

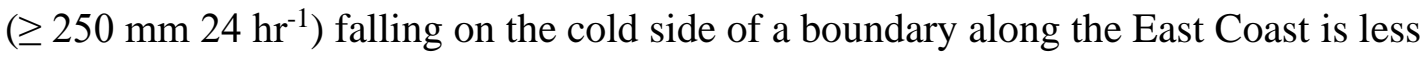
likely: 7 of the 21 events (33\%). Typically, extreme rain on the cold side of a boundary is due to elevated convection. 33\% of extreme rainfall events along the East Coast likely caused by elevated convection, compared to only $19 \%$ caused by landfalling tropical cyclones, makes a study of elevated convection and an investigation into the cause of such events a high priority for atmospheric research.

Additionally, 12 of the 21 events (57\%) had upper-level support for precipitation due to the presence of a trough or closed low over the eastern half of the Continental United States (CONUS). This suggests that more than one atmospheric feature could have played a role in more than half of the extreme rainfall events. Rainfall of this magnitude leads to flash flooding and in some cases, significant floods for large regions that can last several days. It is important to understand these atmospheric features and how they interact to create these events, producing a great threat to life and property on the East Coast. 


\subsection{Observational Analysis}

\subsubsection{South Carolina Flood}

\subsubsection{Hurricane Joaquin}

Originating as a weak mid- to upper-tropospheric low southwest of the Canary Islands on 08 September, a surface low pressure system was labeled a tropical depression 360 nautical miles northeast of San Salvador Island, Bahamas at 0000 UTC 28 September 2015 (Berg 2016). Moderate northwesterly shear kept the system from developing further until high pressure to the north and low pressure to the east of the system forced it southwest (Fig. $2.2 \mathrm{a}$ ) over $30^{\circ} \mathrm{C}$ waters near the Bahamas. Termed a tropical storm at 0000 UTC 29 September 2015 with a central pressure of $1002 \mathrm{hPa}$ (Fig. 2.2b) and a maximum wind speed of 35 knots (Fig. 2.2c), the system rapidly intensified over the warm waters and became a hurricane at 0600 UTC 30 September 2015 with a central pressure of $978 \mathrm{hPa}$ (Fig. 2.2b) and maximum wind of 65 knots (Fig. 2.2c). By 0000 UTC 01 October 2015, Joaquin was a major hurricane with wind of 100 knots just east of San Salvador (Fig. 2.2).

Around 1200 UTC 01 October 2015, Hurricane Joaquin began to turn north over the Bahamas (Fig. 2.2a) and an upper-level trough over the eastern United States (Fig. 2.3a) was beginning to strengthen as the ridge over the western Atlantic Ocean weakened. Joaquin weakened briefly on 02 October 2015 as it was making the turn north, but it underwent a second rapid intensification period on 03 October 2015 to become its 


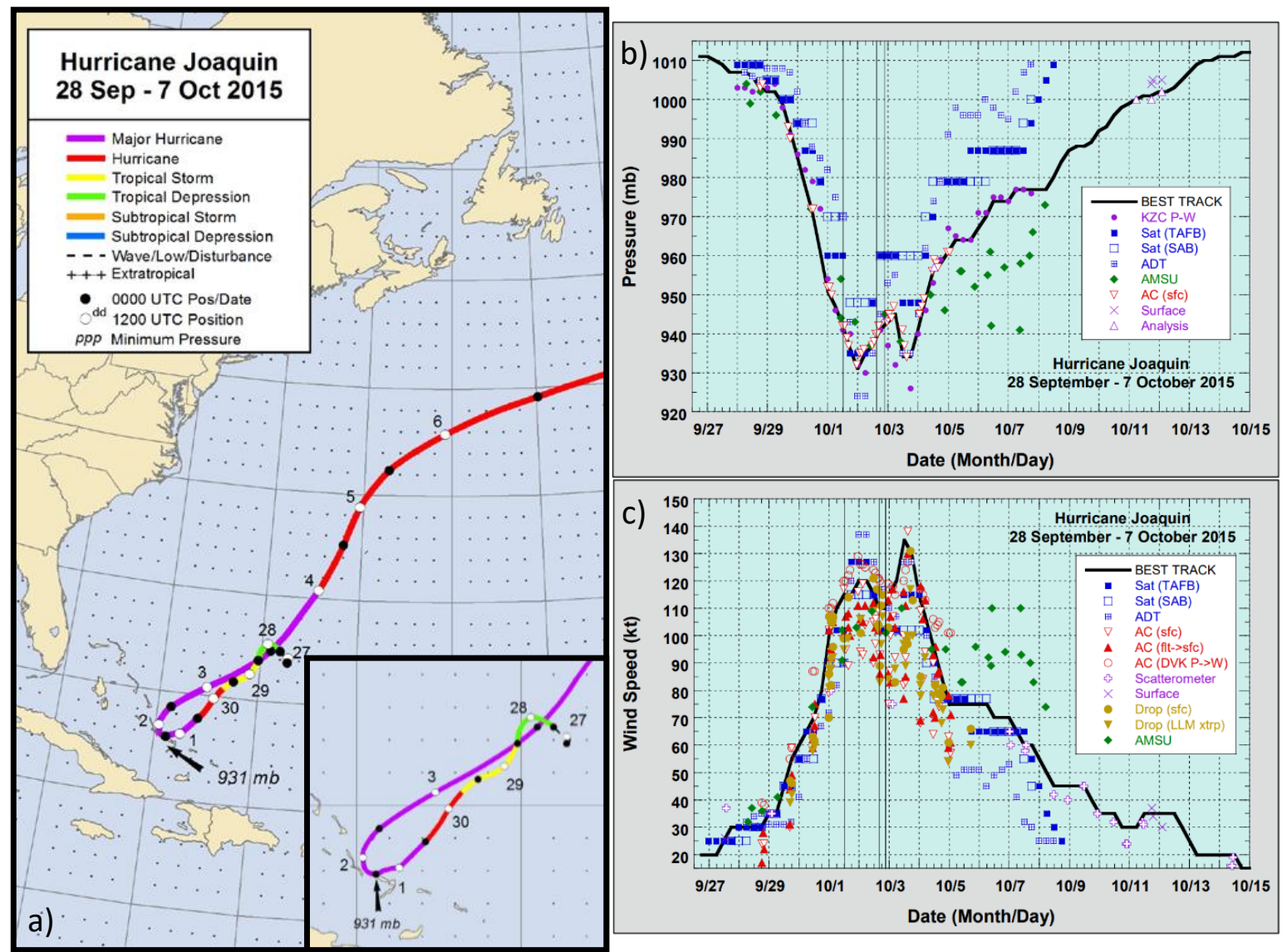

Figure 2.2. Hurricane Joaquin best track a) positions and rank, b) minimum central pressure, and c) maximum sustained surface wind speed covering the period of Joaquin's hurricane status from 28 September 2015 to 07 September 2015 (Berg 2016).

strongest (high-end category 4) at 1200 UTC 03 October 2015 with a central pressure of $934 \mathrm{hPa}$ (Fig. 2.2b) and a maximum sustained wind of 135 knots (Fig. 2.2c). This second period of rapid intensification was likely due to the interaction with the upper-level trough over the eastern United States and the removal of the ridge to the north over the western Atlantic. Joaquin was able to travel north-northeast towards Bermuda, gradually weakening due to northwesterly shear. The hurricane continued to move away from the United States (Fig. 2.2a), became a tropical storm on 07 October 2015, and 
a)

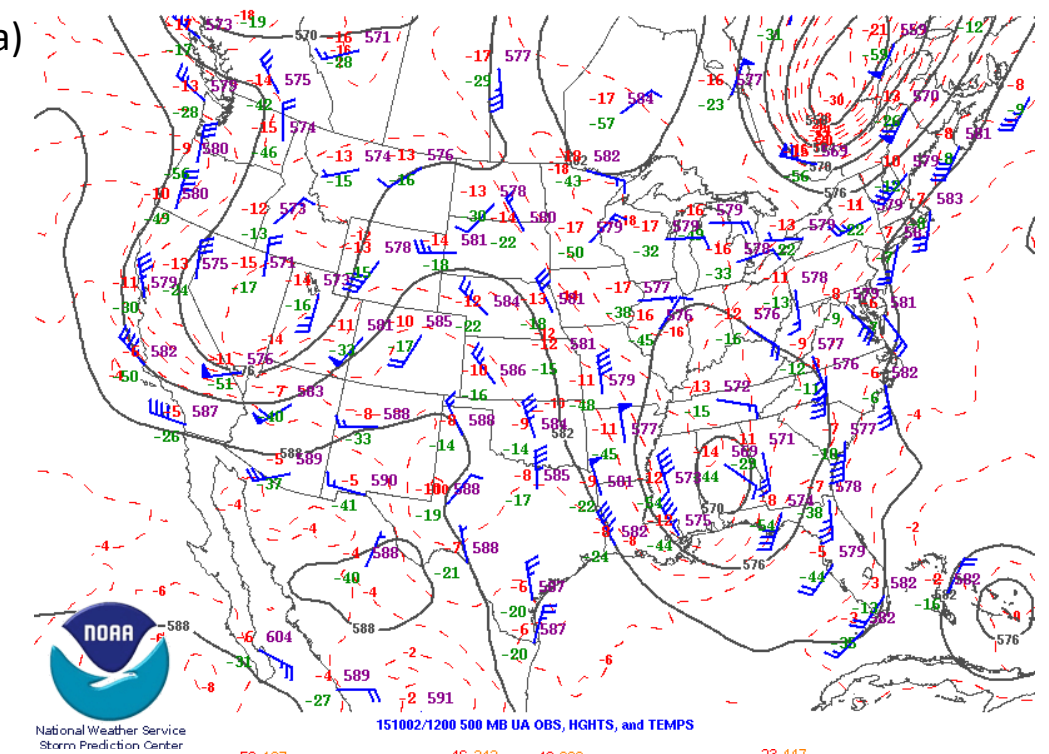

b)

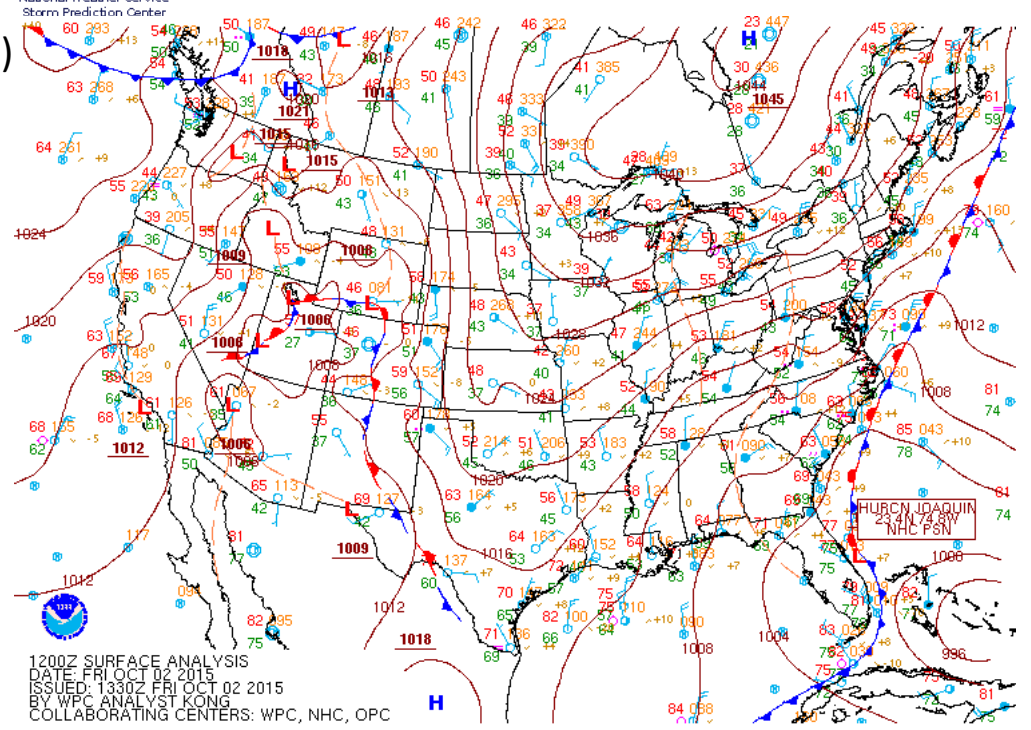

Figure 2.3. National Oceanic and Atmospheric Administration (NOAA) 1200 UTC 02 October 2015 a) 500 $\mathrm{hPa}$ analysis displaying an extratropical cyclone centered over Alabama with Hurricane Joaquin near the Bahamas and b) surface analysis depicting a stationary frontal boundary along the East Coast of the United States.

eventually completed extratropical transition on 09 October 2015 after merging with a frontal boundary over the northeastern Atlantic Ocean (Berg 2016). 


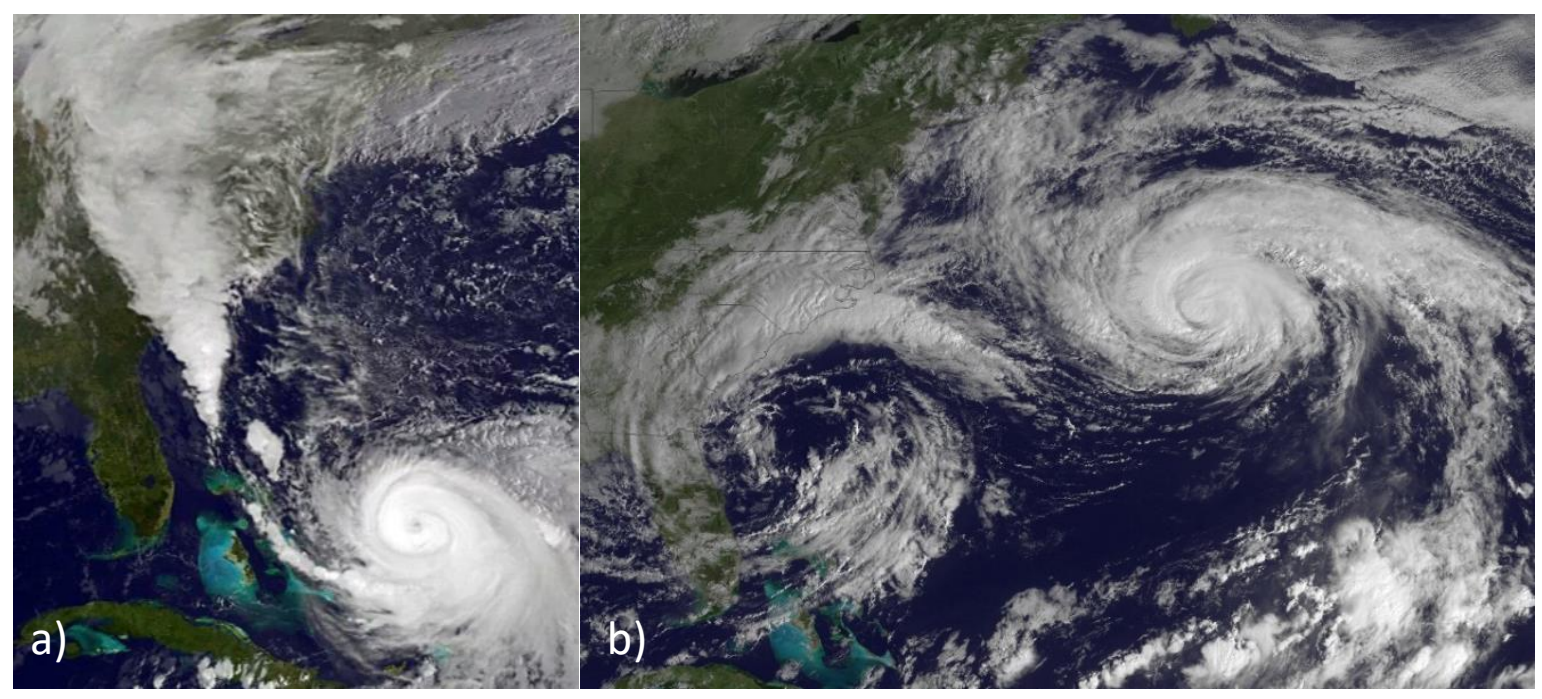

Figure 2.4. Visible satellite images from NOAA's GOES-East of Hurricane Joaquin and the associated moisture plume directed towards South Carolina at a) 1200 UTC 03 October 2015 and b) 1200 UTC 05 October 2015.

\subsubsection{Synoptic}

The upper-level trough deepened over the eastern United States on 01 and 02

October 2015, while Hurricane Joaquin was beginning to move north from the Bahamas.

By 0000 UTC 02 October 2015, an upper-level low was present over the Ohio River

Valley contoured at 5760 meters and 12 hours later a deeper low was centered over

Alabama contoured at 5700 meters (Fig. 2.3a). The low became cut off by 1200 UTC 03

October 2015 over Alabama and the Florida panhandle. The position of the upper-level

low over the southeastern United States in association with Hurricane Joaquin near the

Bahamas (Fig. 2.3b) led to a deformation zone over the Atlantic Ocean east of Florida.

The axis of dilatation associated with that deformation zone allowed for convergence of moisture and a direct flow towards South Carolina (Fig. 2.4a). As Joaquin moved 


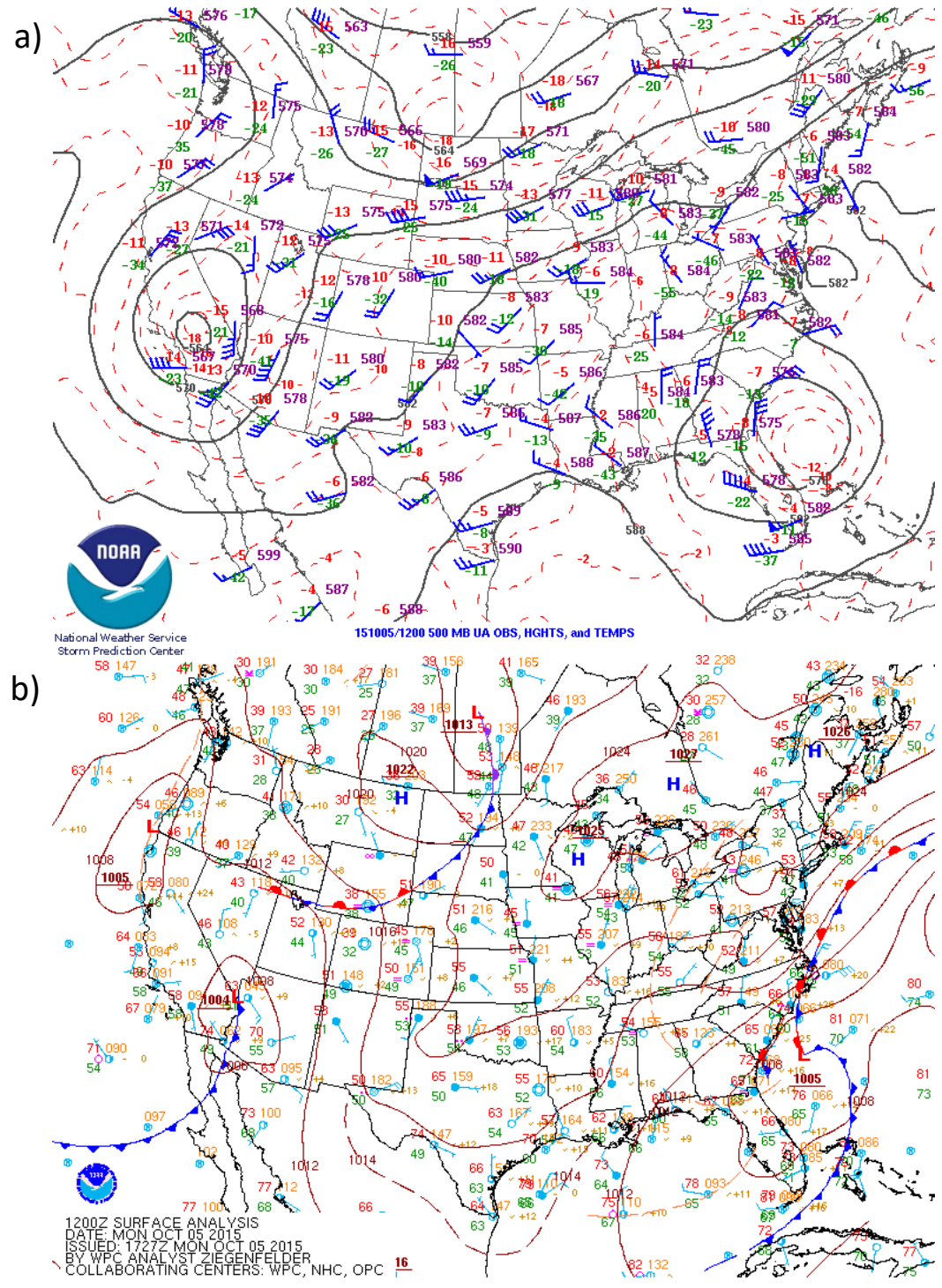

Figure 2.5. As in Fig. 2.3, except 1200 UTC 05 October 2015.

northeast, the cut-off low began to rotate counterclockwise placing South Carolina in the right entrance region of the associated jet streak (not shown) and keeping the axis of dilatation directed at the area (Fig. 2.4b). The upper-level low slowly pushed offshore by 


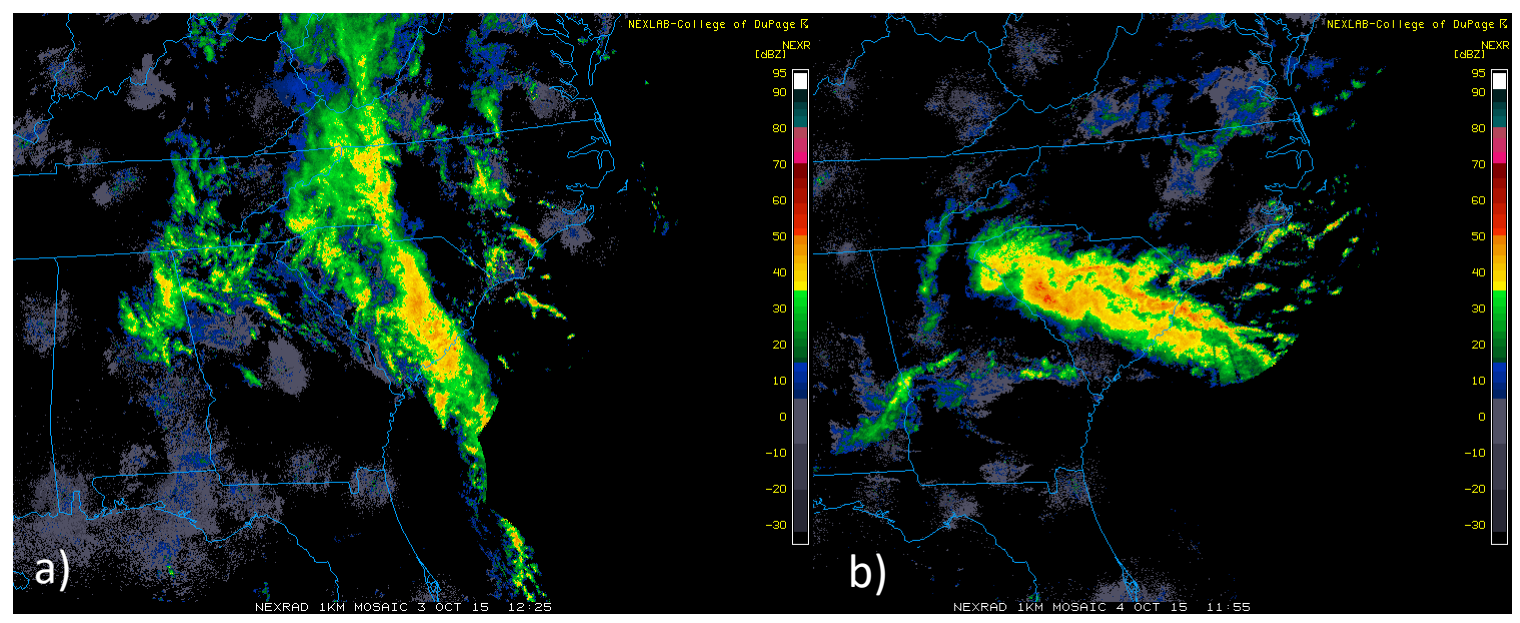

Figure 2.6. Radar reflectivity (dBZ) mosaic from College of DuPage Next Generation Weather Lab over the southeastern United States at a) 1225 UTC 03 October 2015 and b) 1155 UTC 04 October 2015.

1200 UTC 05 October 2015 (Fig. 2.5a) and proceeded to follow Hurricane Joaquin northeast over the Atlantic Ocean (Fig. 2.4b).

\subsubsection{Mesoscale}

A cold front stretched from eastern Canada, across New England, and west to the Texas panhandle at 1200 UTC 30 September 2015 made its way east and was draped along the East Coast of the United States by 0000 UTC 01 October 2015. The front stalled just offshore (Fig. 2.3b), where it remained for the entirety of the heavy rainfall event. By 1200 UTC 05 October 2015, the upper-level low (Fig. 2.5a) and a surface low off the coast of South Carolina (Fig. 2.5b) begin to alter the boundary, forming a cold front and pushing east. 


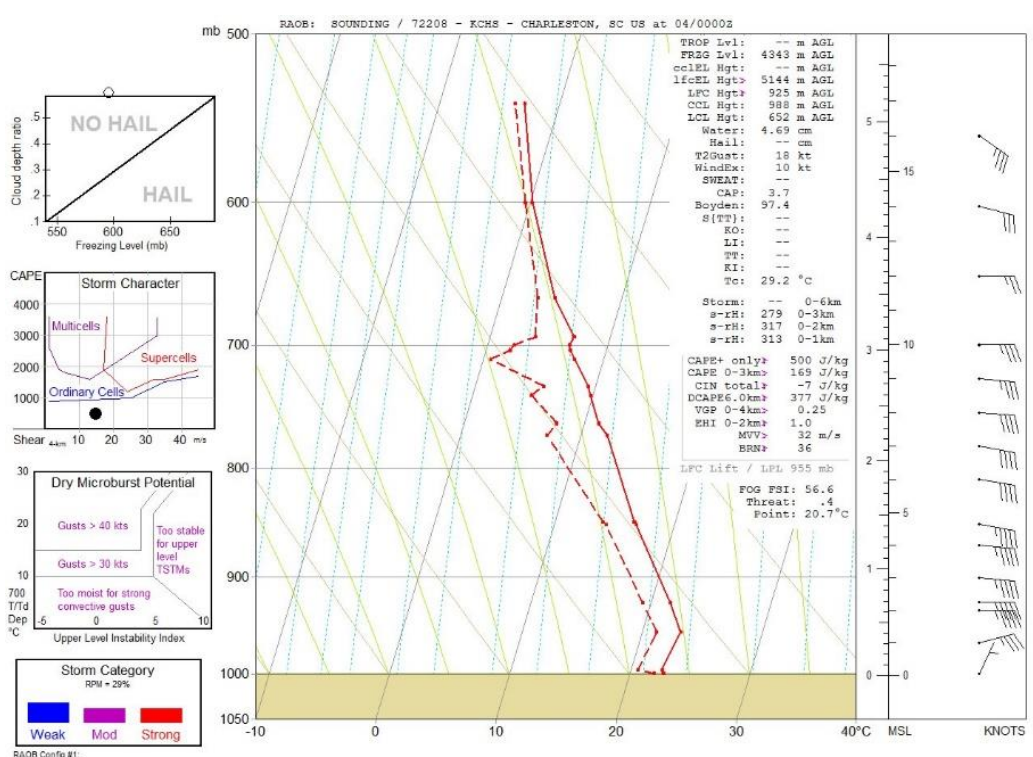

Figure 2.7. Observed sounding from Charleston, South Carolina National Weather Service (NWS) at 0000 UTC 04 October 2015 obtained by The Universal Rawinsonde Observation program (RAOB).

The surface low depicted in Figure 2.5b was located near Savannah, Georgia from 1200 UTC 03 October through 0600 UTC 04 October 2015, which corresponds with the time of heaviest rainfall for South Carolina. The position of the surface low corresponded with the position of the upper-level low and the deformation zone, allowing the directed flow of moisture over the stationary front and into South Carolina. Radar images show the orientation of heavy rain from 1200 UTC 03 October to 1200 UTC 04 October 2015 over South Carolina (Fig. 2.6), as the deformation zone shifts with the movement of Hurricane Joaquin the axis of precipitation rotates from southeasterly to easterly.

With flow of moisture perpendicular to a frontal boundary and rainfall occurring on the cold side of the boundary, it is possible that the frontal boundary initiated 


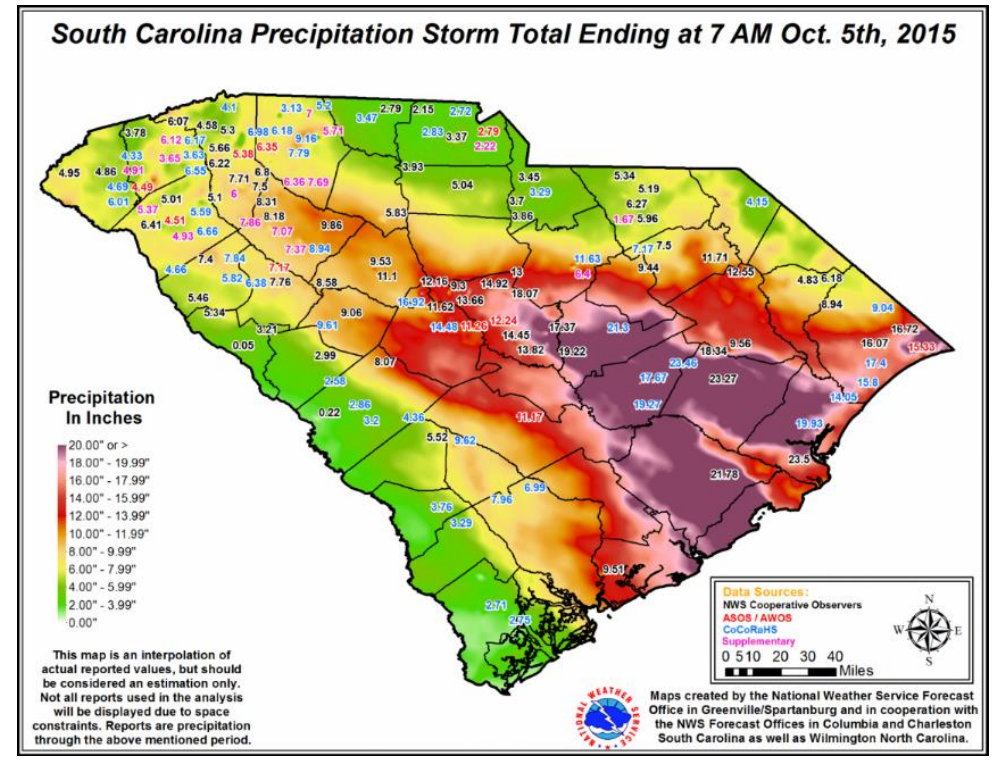

Figure 2.8. Total observed precipitation (inches) for South Carolina from 0000 UTC 01 October 2015 to 1100 UTC 05 October 2015. Image courtesy of NWS offices in South Carolina and North Carolina.

convection by lifting parcels to the level of free convection. The observed temperature profile from 0000 UTC 04 October 2015 at Charleston, South Carolina shows a slight temperature inversion, suggesting the convection at that time to be elevated in nature (Fig. 2.7). Elevated convection has been associated with intense precipitation (e.g., Rochette and Moore 1996; Moore et al. 1998; McCoy et al. 2017), so it is no surprise that observations from this event suggest elevated convection at the time of heaviest rainfall.

\subsubsection{Forecast and Impacts}

Numerical weather prediction (NWP) models were able to hint at a significant rainfall event for South Carolina as early as 30 September 2015 when NOAA's Weather 


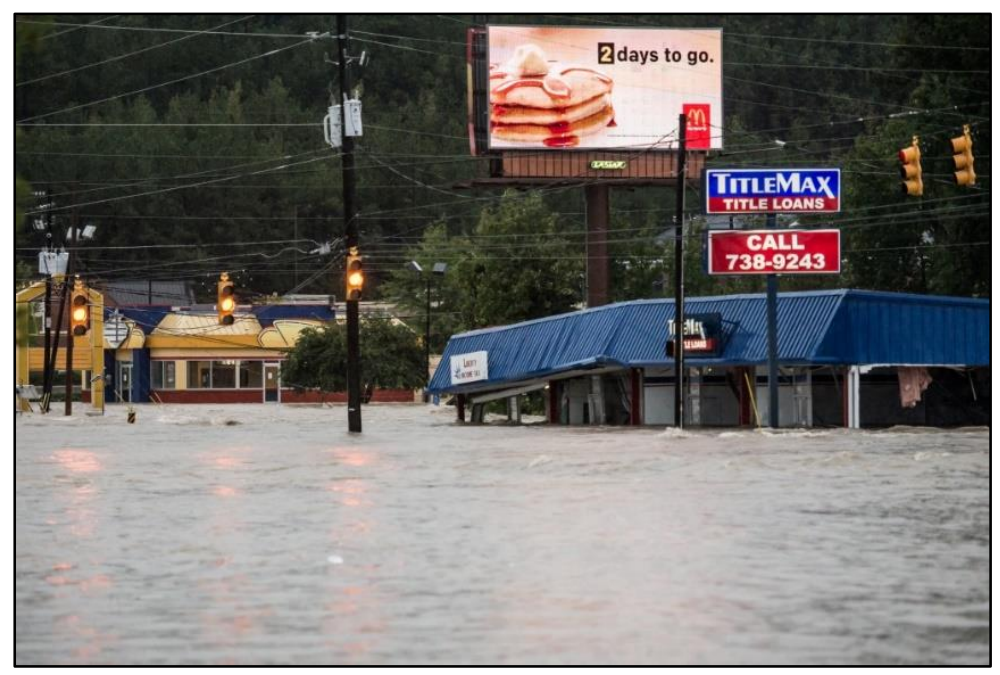

Figure 2.9. Image of the flood waters near Columbia, South Carolina as a result of the heavy rainfall in early October 2015. Image from the NWS Columbia, SC Event Review of the 2015 South Carolina Flood.

Prediction Center (WPC) first acknowledged the threat (Murphy 2016). Moderate Excessive Rainfall Outlooks were provided for South Carolina 2 and 3 days out, with the High Risk for flash flooding posted on the Day 1 outlook. Models were in good agreement on 01 October 2015 that the rainfall event on 04 October would exceed 10 inches over South Carolina. WPC's precipitation forecast verified well for the entire event stretching from 01-05 October 2015 (Murphy 2016). The event ended with a large portion of eastern South Carolina totaling over 20 inches of rain in just over 4 days (Fig. 2.8). The heaviest rain occurred near Mount Pleasant, South Carolina, which received the heaviest rain around 0000 UTC 04 October 2015 with more than 10 inches in a 24-hour period ( 1200 UTC 03 October 2015 to 1200 UTC 04 October 2015) and a total of 26.88 inches $(682.75 \mathrm{~mm})$ of rain for the entire event (Berg 2016). 
Charleston International Airport in South Carolina also received more than 10 inches of rain in a 24-hour period, with 11.5 inches falling on 03 October 2015 (Berg 2016). The one-, two-, three-, and four-day rainfall records were broken during this event at the airport, with an event total of 17.29 inches. The heavy rain led to flooding for a large part of South Carolina, with Columbia Metro Airport also setting rainfall records. The flooding from rainfall in the Charleston area was exacerbated by high tides, preventing the runoff from entering Charleston Harbor (Berg 2016). Thousands of homes, businesses, roads, bridges, and dams were damaged or destroyed by flood waters in South Carolina (Fig. 2.9). According to the National Weather Service (NWS) office in Columbia, South Carolina, numerous life-saving rescues were performed in the swift waters.

While one goal of this study is to improve forecasts and warning lead time, it is hard to imagine the South Carolina flood having a better forecast. The primary goal of this research is to understand the cause of the heavy, prolonged rainfall. A better scientific understanding of the event, considering the number of meteorological features in play, will improve forecasts of similar events in the future that may or may not contain all the same features if models were to fail with such events. Additionally, that scientific understanding could be implemented into the development of NWP models in hopes of getting nearly unanimous forecast rainfall amounts from them 4 or more days, rather than 3 days, in advance. 


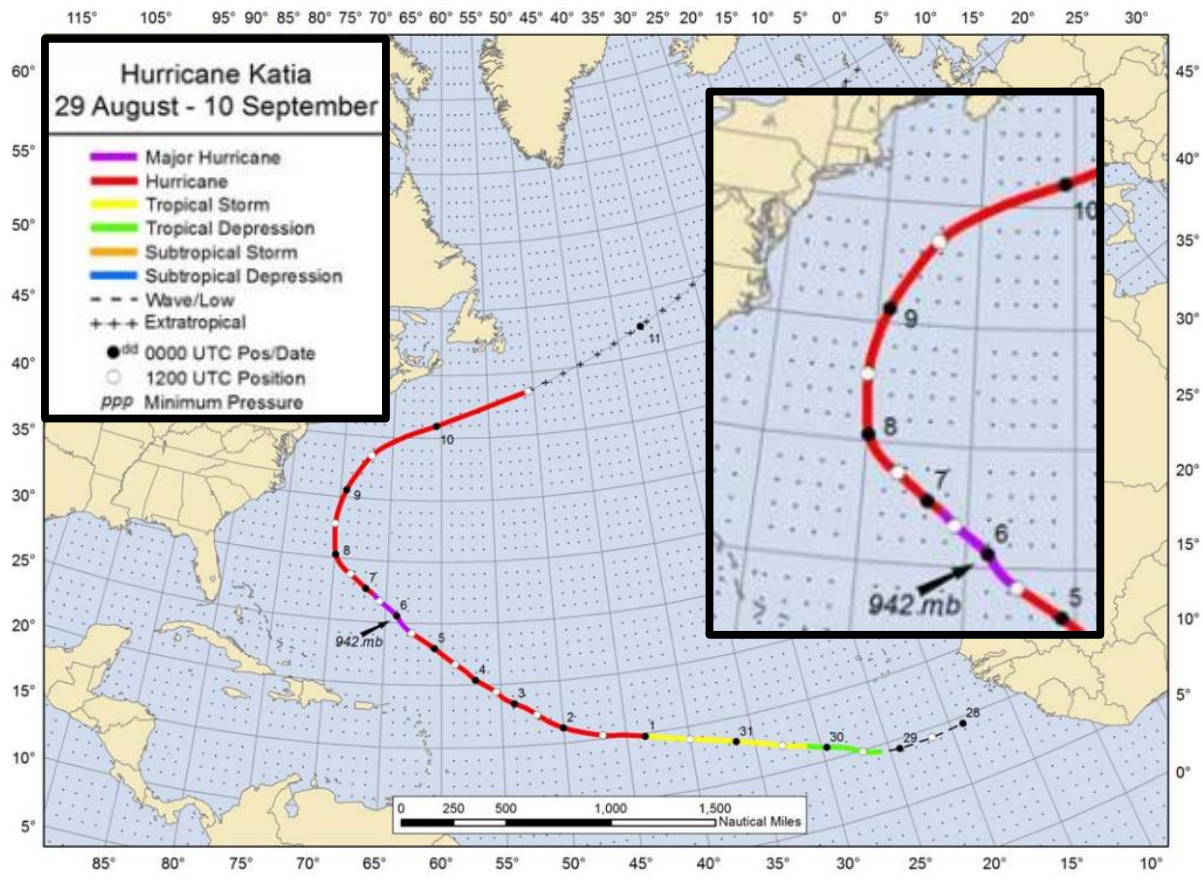

Figure 2.10. Hurricane Katia best track positions and rank from 29 August 2011 to 10 September 2011 (Stewart 2012).

\subsubsection{September 2011 Flood}

\subsubsection{Hurricane Katia}

Beginning as a tropical wave on 27 August 2011 off the west coast of Africa, Katia became a tropical depression by 0600 UTC 29 August 2011 (Stewart 2012). It was termed a tropical storm around 0000 UTC 30 August 2011 while located roughly 425 nautical miles southwest of the Cape Verde Islands (Fig. 2.10). With vertical shear decreasing, Katia was able to move west-northwest at about 15 knots and gradually strengthen for two days, becoming a hurricane by 0000 UTC 01 September 2011. A large, subtropical ridge over the Atlantic allowed Hurricane Katia to move northwestward, but an increase in vertical wind shear kept Katia from strengthening 

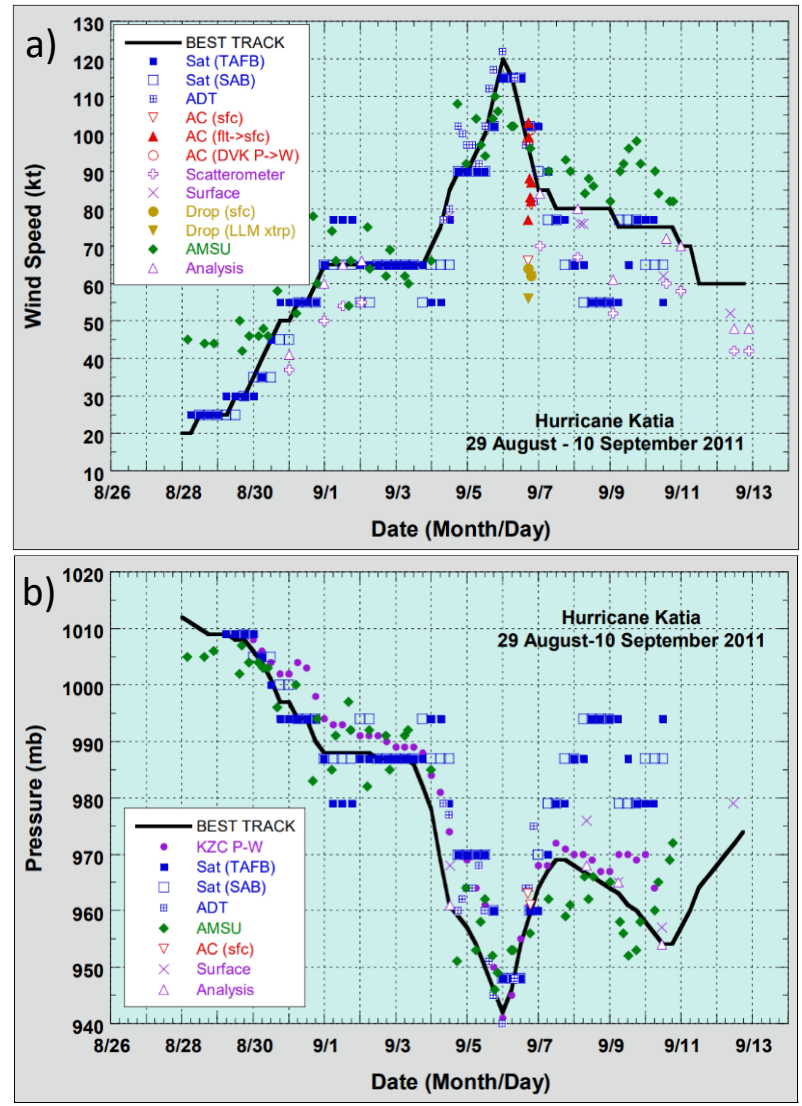

Figure 2.11. Hurricane Katia best track a) maximum sustained surface wind speed and $b$ ) minimum central pressure from 28 August 2011 to 13 September 2011 (Stewart 2012).

further (Stewart 2012). It was not until 0000 UTC 04 September 2011 that the vertical wind shear began to decrease and Katia began to intensify. Rapidly intensifying, Katia reached major hurricane status at 1200 UTC 05 September 2011 (Fig. 2.10). The cyclone was its strongest with winds of 120 knots (Fig. 2.11a) and a central pressure of $942 \mathrm{hPa}$ (Fig. 2.11b) at 0000 UTC 06 September 2011, 470 nautical miles south of Bermuda (Fig. 2.10).

After becoming its strongest, Hurricane Katia began to rapidly weaken and slow down due to an eyewall replacement cycle as it interacted with a deep-layer trough over 


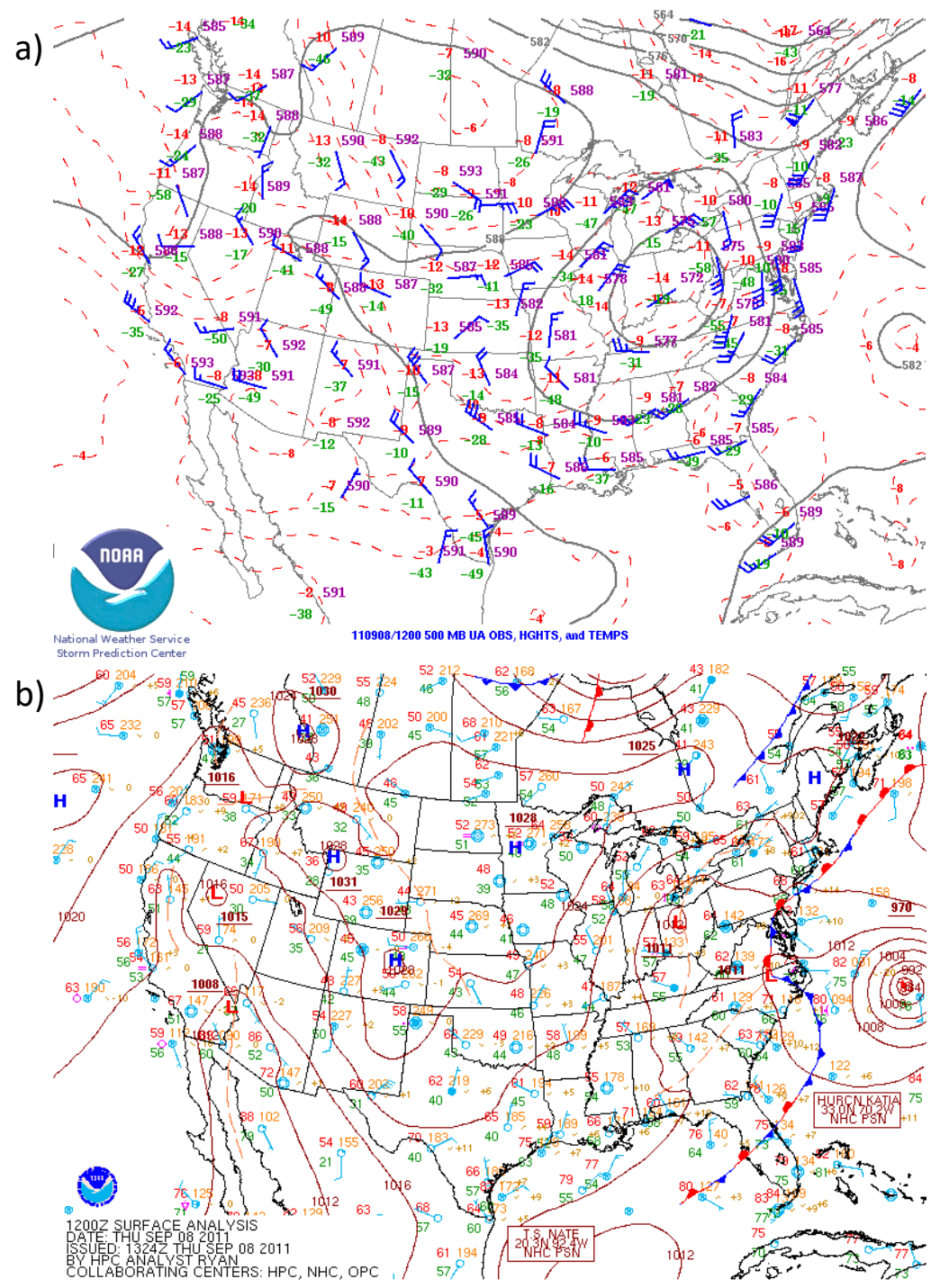

Figure 2.12. NOAA 1200 UTC 08 September 2011 a) 500-hPa analysis displaying an extratropical cyclone centered over the Ohio River Valley with Hurricane Katia near Bermuda and b) surface analysis depicting a surface low and associated frontal boundary along the East Coast of the United States.

the eastern United States (Stewart 2012). The trough provided steering flow for Katia to move north on 08 September 2011 and then northeast on 09 September 2011, preventing landfall on the East Coast of the United States (Fig. 2.10). After 1200 UTC 09 September 2011, Hurricane Katia began to accelerate east-northeast toward the north Atlantic. 


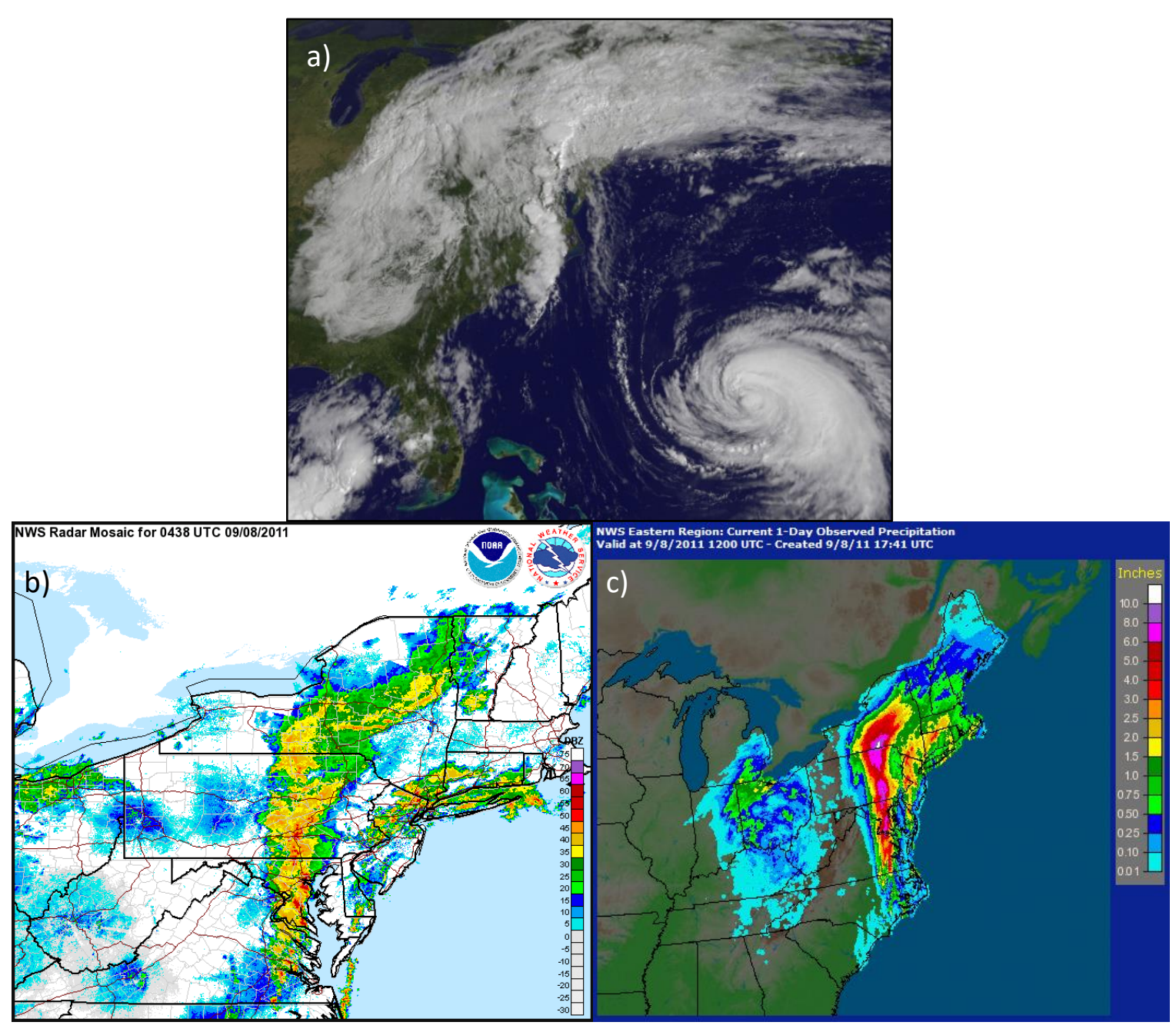

Figure 2.13. Northeastern United States a) visible satellite image from NOAA's GOES-East of Hurricane Katia and the moisture plume directed towards Pennsylvania at 1400 UTC 07 September 2011, b) NWS radar reflectivity (dBZ) mosaic at 0438 UTC 08 September 2011, and c) NWS derived total rainfall (inches) for the 24-hour event from 1200 UTC 07 September 2011 to 1200 UTC 08 September 2011.

\subsubsection{Pattern for Precipitation}

The mid-level trough began to enter the region of the eastern United States on 05 September 2011, while Tropical Storm Lee was beginning to weaken over Louisiana. As Tropical Storm Lee pushed northeast toward the mid-Atlantic region of the United States, the two systems merged and a cut off low was present in the mid-levels over the 
southeast United States. Together, the system marched northeast until the synoptic pattern across the region stalled the systems on 07 September 2011. For a solid 24-hour period (1200 UTC 07 September to 1200 UTC 08 September 2011), the mid-level low was located over the Ohio River Valley (Fig. 2.12a) and a surface low was strengthening over North Carolina, with associated frontal boundaries stretching along the East Coast of the United States (Fig. 2.12b). All the while, Hurricane Katia was churning off the coast between the surface low and Bermuda (Fig. 2.12).

The locations of all involved systems allowed for a moisture plume to develop from coastal Virginia to central New York before 1400 UTC 07 September 2011 (Fig. 2.13a) and continued well after 0438 UTC 08 September 2011 (Fig. 2.13b). The result of this moisture plume was a narrow margin of more than 5 inches of rain stretching from Virginia to New York, with more than 10 inches of rain falling on locations in Pennsylvania and New York (Fig. 2.13c), in just the 24-hour period from 1200 UTC 07 September to 1200 UTC 08 September 2011. Moisture from the remnants of Tropical Storm Lee was a big factor in the copious amounts of rainfall that fell over the northeast region. The synoptic pattern, combined with a surface frontal boundary, was likely the primary reason for the flooding in Pennsylvania and New York (Gitro et al. 2014). Moisture from remnants of Tropical Storm Lee alone would have provided plenty of rainfall for the region, but the significant amounts of more than 10 inches in 24 hours were possible because the system stalled. The interaction of all systems, including Hurricane Katia, led to the stagnant pattern and stationary front, providing a consistent flow of moisture over the impacted area for a considerable amount of time until all systems were able to continue their trek northeast. 


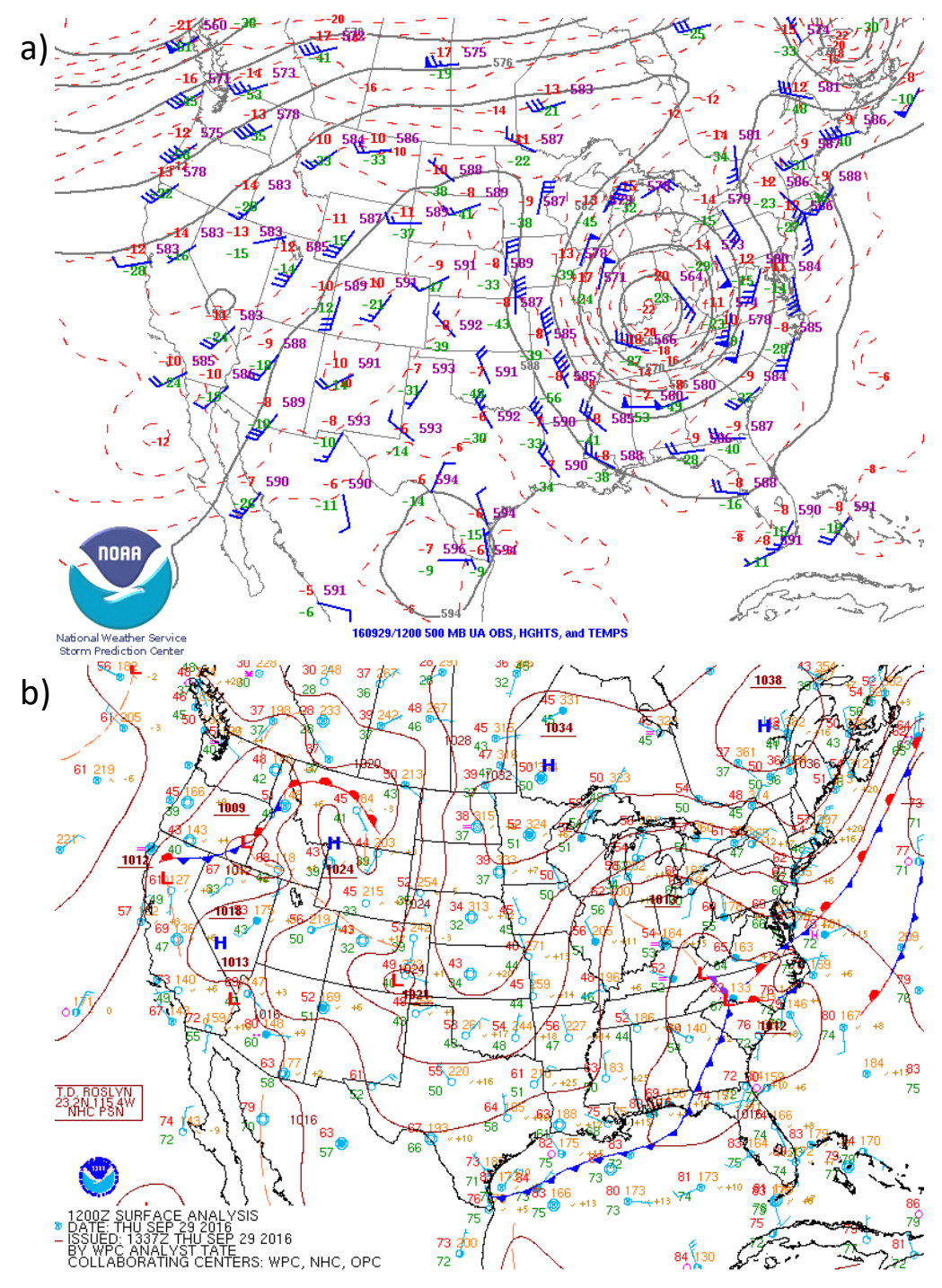

Figure 2.14. NOAA 1200 UTC 29 September 2016 a) 500-hPa analysis displaying an extratropical cyclone centered over the Ohio River Valley and $b$ ) surface analysis depicting a surface low and associated frontal boundary along the East Coast of the United States.

\subsubsection{September 2016 - North Carolina}

Areas near Fayetteville, North Carolina received 10 inches of rain between 1200

UTC 28 September to 1200 UTC 29 September 2016. This significant amount of

precipitation was the result of an upper-level closed low (Fig. 2.14a) and a surface low 


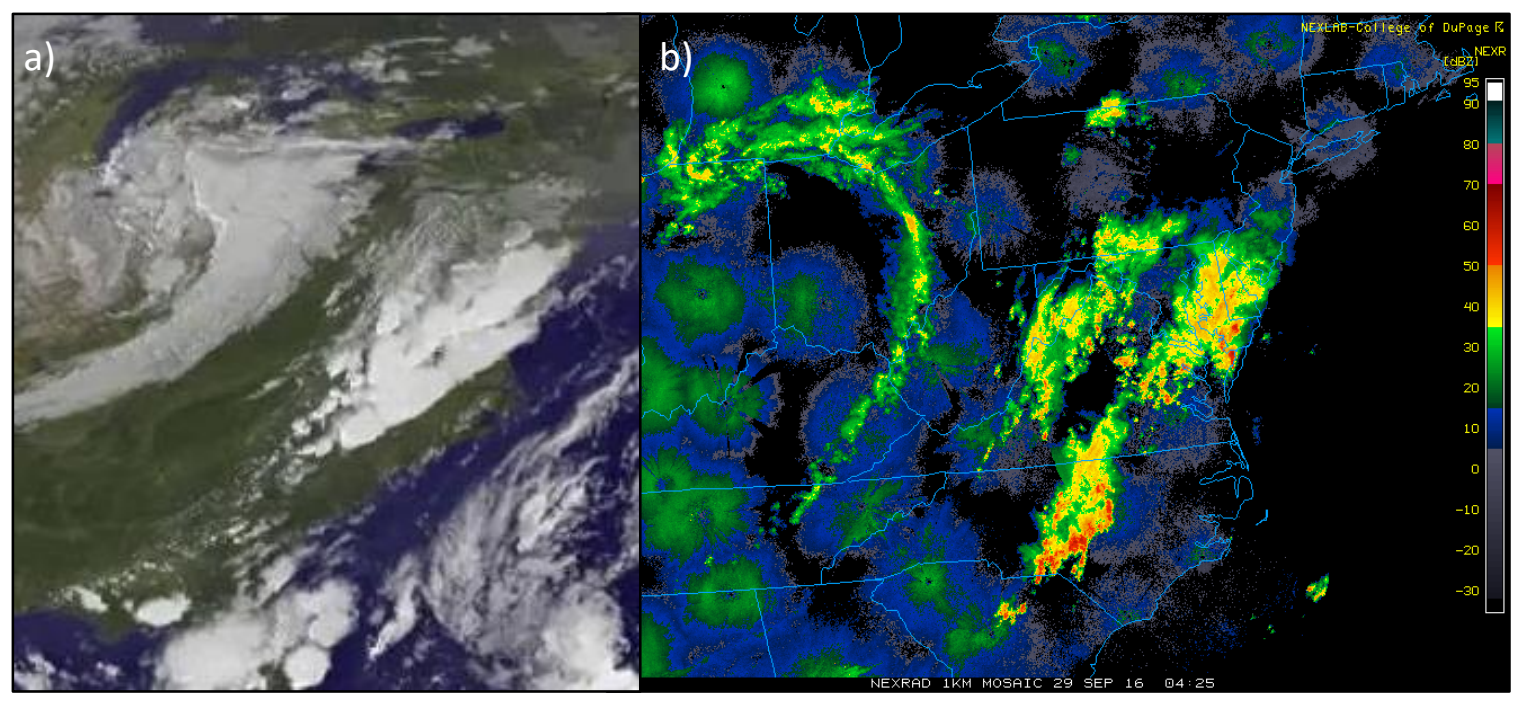

Figure 2.15. East Coast United States a) visible satellite image from NOAA's GOES-East at 2215 UTC 28 September 2016 and b) radar reflectivity (dBZ) mosaic from College of DuPage Next Generation Weather Lab at 0425 UTC 29 September 2016 depicting convection over North Carolina and Virginia.

with associated frontal boundaries (Fig. 2.14b). Plenty of moisture flowed into the region from the Gulf of Mexico and the Atlantic Ocean due to the southerly flow. Additionally, the coastal front allowed for the development of cold air damming over west-central North Carolina. This setup suggests the possibility of elevated convection in the region of a cold, stable surface layer.

The convection over North Carolina appeared in satellite images to be associated with a focused plume of moisture (Fig. 2.15a). A line of thunderstorms across central North Carolina developed, with one forming behind another and then pushing north (Fig. $2.15 b)$. The storms were intense enough to provide high rainfall rates, but the synoptic and mesoscale patterns maintained the production of thunderstorms in the same location for several hours to supply over 10 inches to parts of North Carolina over the entirety of the event. 

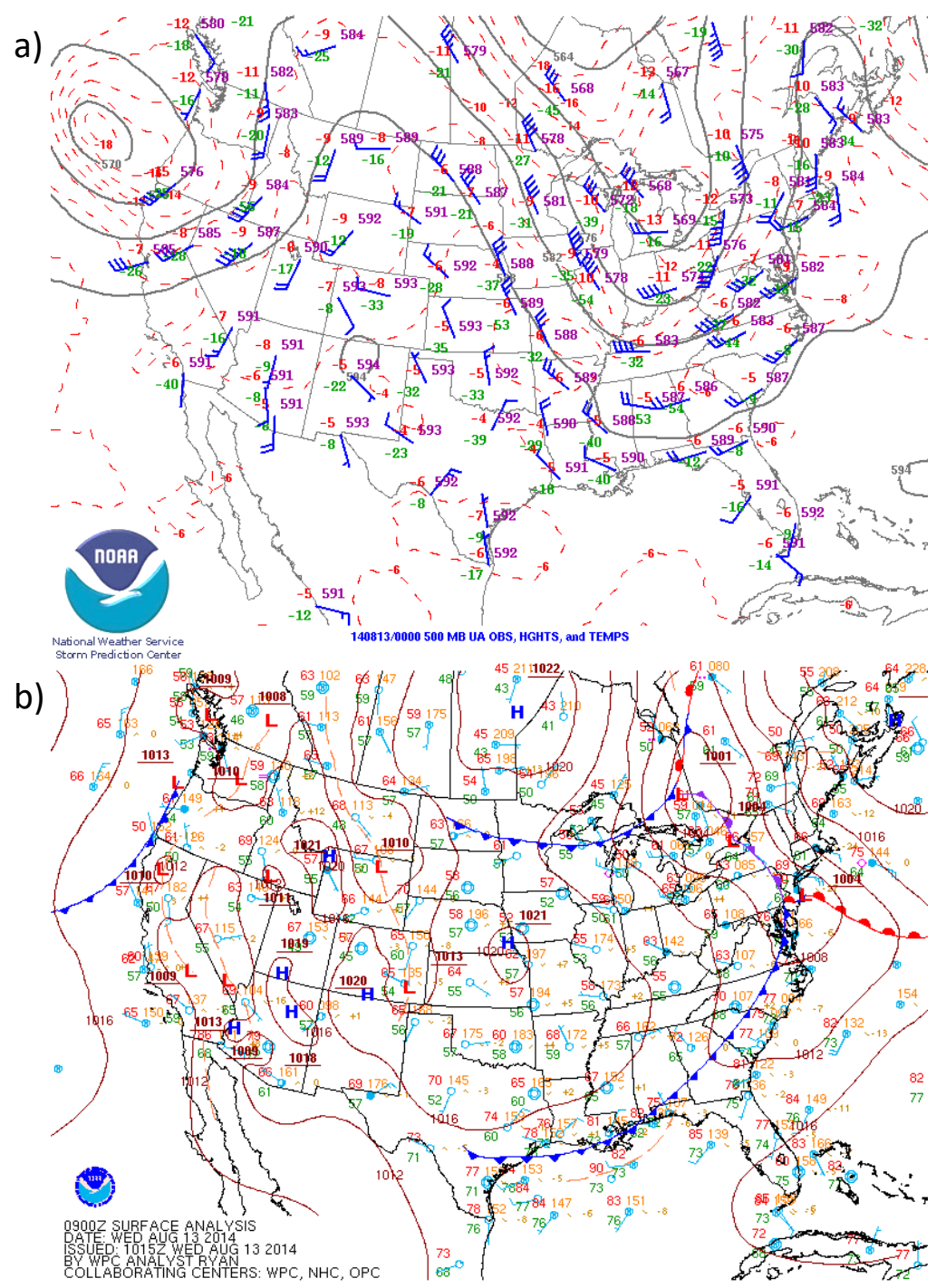

Figure 2.16. NOAA a) 0000 UTC 13 August 2014 500-hPa analysis displaying a trough over the eastern United States and b) 0900 UTC 13 August 2014 surface analysis depicting a surface low and associated frontal boundaries along the East Coast of the United States.

\subsubsection{August 2014 - New York}

On 13 August 2014, an upper-level trough was situated over the eastern half of North America (Fig. 2.16a) and a surface low was located near New York City (Fig. 2.16b). The pattern is typical of a rainfall event for region of the surface low pressure. 


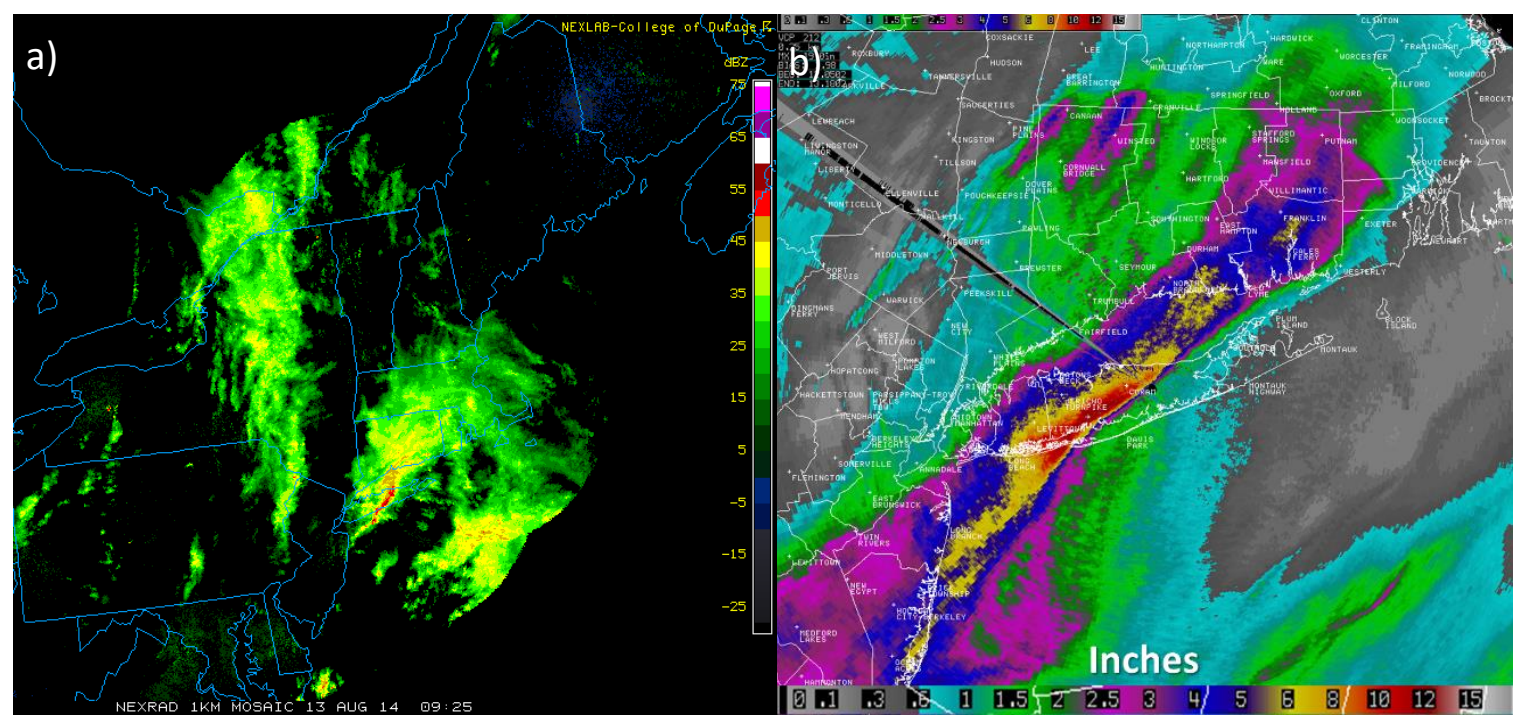

Figure 2.17. Northeastern United States a) radar reflectivity (dBZ) mosaic from College of DuPage Next Generation Weather Lab at 0925 UTC 13 August 2014 and b) NWS radar estimated total precipitation (inches) from 0500 UTC 12 August 2014 to 1800 UTC 13 August 2014 showing a maximum total of 19 inches for Long Island, New York and large area with greater than 10 inches of rain.

Not seen from the NOAA analyses (Fig. 2.16) is the strong radar reflectivity over Long Island, New York at 0925 UTC 13 August 2014 (Fig. 2.17a). This intense convection led to a record breaking 13.27 inches of rain in Islip, New York (Fig. 2.17b), recorded by the Islip Airport Automated Surface Observing Station (ASOS). The record broke the New York State 24-hour precipitation record, previously set by the landfalling Hurricane Irene in 2011. However, the rainfall on 13 August 2014 was not caused by a landfalling tropical cyclone, but rather the synoptic pattern transporting deep moisture over the region and the warm front associated with the surface low sitting just south of Long Island. The orientation of the warm front, parallel to the overall storm motion, allowed for the copious amounts of rain to fall in just a few hours. According to the New York NWS, 1.08 inches of rain fell in just 9 minutes from 0939 to 0947 UTC 13 August 2014. 


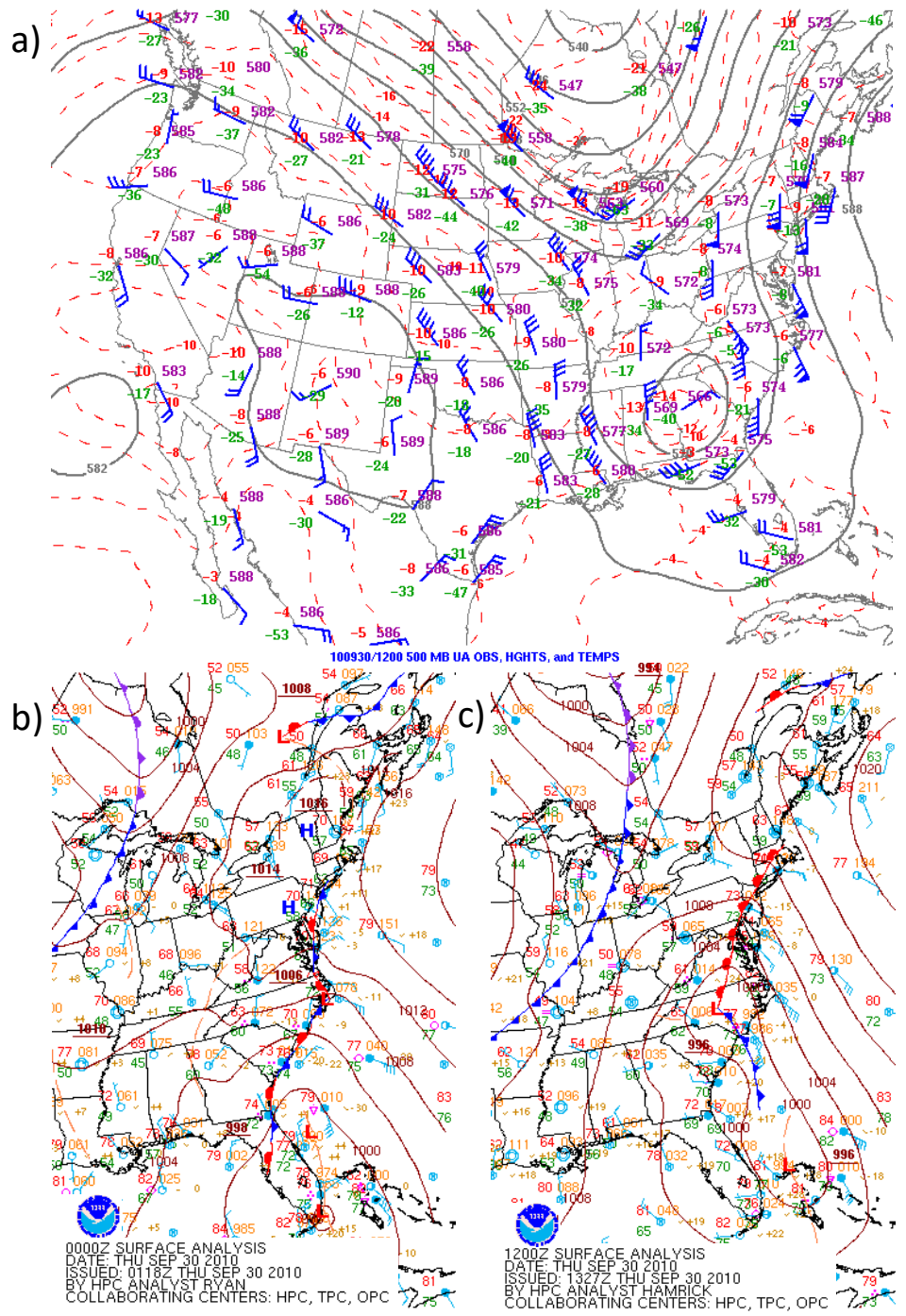

Figure 2.18. NOAA a) 1200 UTC 30 September 2010 500-hPa analysis displaying an extratropical cyclone over Georgia, b) 0000 UTC 30 September 2010 surface analysis depicting a stationary front along the East Coast of the United States, and c) 1200 UTC 30 September 2010 surface analysis depicting a surface low and associated frontal boundaries in North Carolina.

\subsubsection{September 2010 - North Carolina}

The coastal regions of North Carolina were the target of an extreme rain event on 30 September 2010. An upper-level low pressure system over the southeastern United

States was associated with a negatively tilted trough over the eastern half of North 


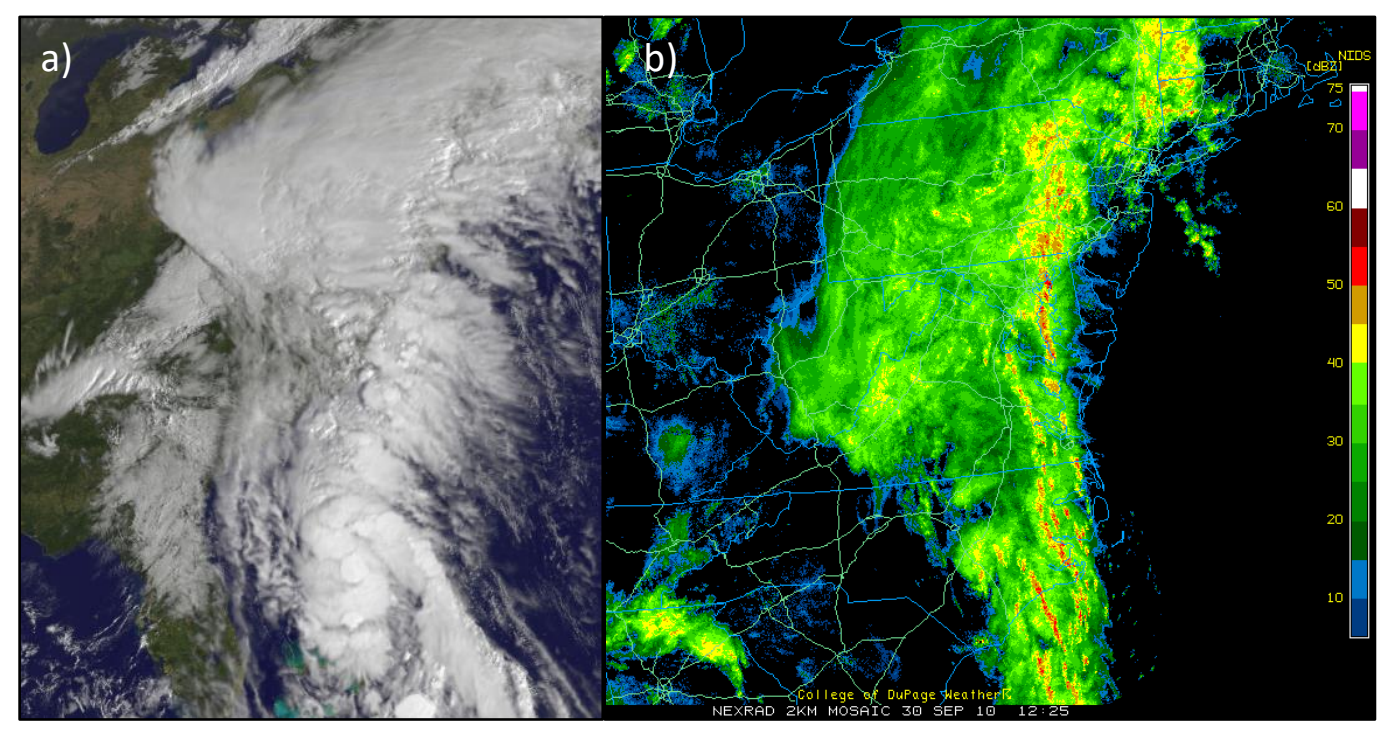

Figure 2.19. East Coast United States a) visible satellite image from NOAA's GOES-East at 1325 UTC 30 September 2010 and b) radar reflectivity (dBZ) mosaic from College of DuPage Next Generation Weather Lab at 1225 UTC 30 September 2010 showing a north-south oriented line of convection.

America at 1200 UTC 30 September 2010 (Fig. 2.18a). Additionally, a stationary boundary stretched along the East Coast of the United States (Fig. 2.18b) was transitioning into a warm front and a cold front due to the development of a surface low over North Carolina (Fig. 2.18c). That setup alone was enough to begin a heavy rain event with the characteristics of a Maddox "Synoptic" type flood event (Maddox et al. 1979): a stalled frontal boundary, an upper-level trough west of the front, and a moisture plume east of the front (similar to that of the other cases in this study). Added moisture for the event may have also been provided by the remnants of Tropical Storm Nicole as it reached the coast near 0000 UTC 01 October 2010 (Otto 2010), while a blocking ridge over the western Atlantic held the synoptic pattern in place allowing for a longer period of deep southerly flow along the East Coast of the United States (also similar to that of the other cases in this study). 


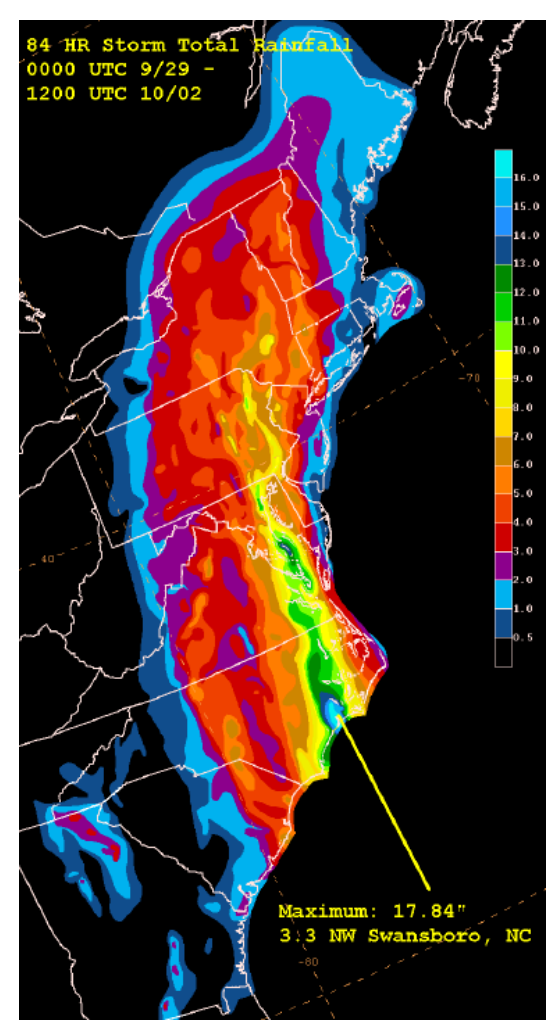

Figure 2.19. NWS derived total rainfall (inches) over the East Coast of the United States from 0000 UTC 29 September 2010 to 1200 UTC 02 October 2010 showing a maximum of 17.84 inches of rain near Swansboro, North Carolina in the 84-hour period.

North Carolina's heaviest rainfall occurred before the arrival of the remnants of Tropical Storm Nicole, while it was located off the coast of southern Florida (Fig. 2.19a), with a continuous line of convection along the East Coast around 1200 UTC 30 September 2010 (Fig. 2.19b). While only an area in coastal North Carolina received 10 inches in a 24-hour period, the complete event lasted for roughly 84 hours and amounted to a large region of the mid-Atlantic and northeastern United States receiving more than 5 inches of rain (Fig. 2.19). Swansboro, North Carolina received the maximum rainfall in this 84-hour event with a total of 17.84 inches (Fig. 2.19). 


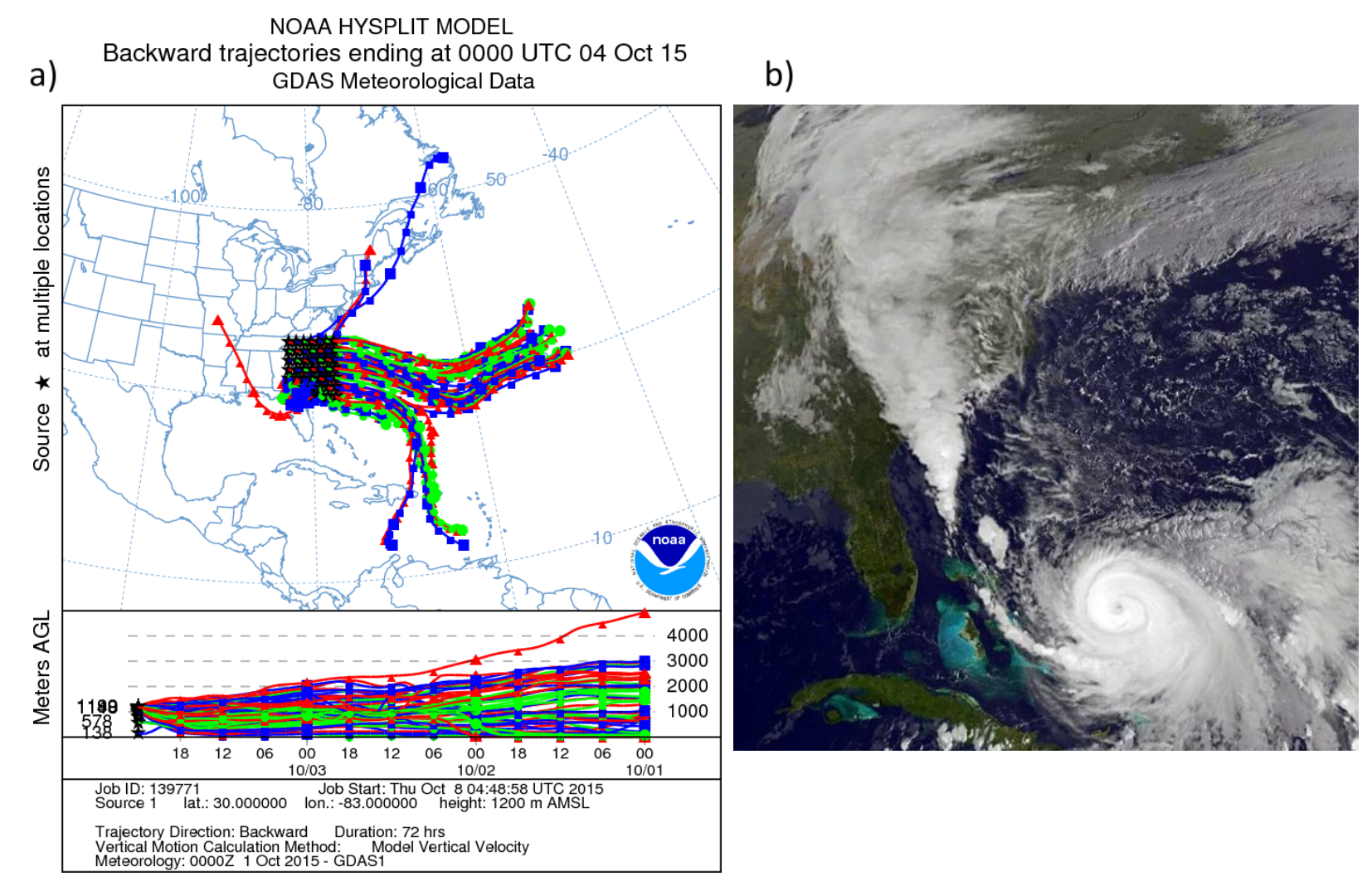

Figure 2.20. The moisture transport associated with the 2015 South Carolina flood event was analyzed using a) the ARL HYSPLIT model to create 72-hour backward trajectories of air parcels located over South Carolina at 0000 UTC 04 October 2015, which was a time of heavy rainfall for the state, and b) visible satellite imagery from NOAA's GOES-East satellite of Hurricane Joaquin and the associated moisture plume at 1200 UTC 03 October 2015.

\subsection{Initial Motivation}

The question of how the tropical cyclone contributed to the 2015 South Carolina flood event was the initial motivation for this study. Preliminary research using the Air Resources Laboratory (ARL) Hybrid Single-Particle Lagrangian Integrated Trajectory (HYSPLIT) model suggested that the presence of the tropical cyclone had a significant influence on the location of the elevated convection, but the cold-core low and the front placement also played important roles. Using the ARL HYSPLIT model, backward 
trajectories for the moisture associated with the South Carolina heavy rainfall event (Fig. 2.20a) were calculated. These trajectories showed that the majority of the moisture in the event originated in the mid-Atlantic Ocean, well east of Hurricane Joaquin, which aids Objective 1 of the proposed research. Hurricane Joaquin's location (Fig. 2.20b) may have helped to transport the moisture towards South Carolina, but was likely not the primary source of moisture for the event. Thus, further investigation was required to determine the cause of the extreme rain event. 


\section{CHAPTER 3. METHODOLOGY}

\subsection{Numerical Modeling}

\subsubsection{Model Overview}

To investigate Hurricane Joaquin's role in the South Carolina flood event further and support Objective 2, a quantitative approach using numerical weather simulations was explored. Using the Weather Research and Forecasting (WRF) model with the Advanced Research WRF (ARW) dynamical core, a simulation of the South Carolina flood event was completed. WRF is an atmospheric modeling system developed by the National Center for Atmospheric Research (NCAR). Numerous physics options are available with this model, which utilizes fully compressible nonhydrostatic equations, domain nesting capabilities, an Arakawa C-grid, and Runge-Kutta $2^{\text {nd }}$ and $3^{\text {rd }}$ order time integration options (Wang et al. 2017). A complete list of model details and key features for WRF-ARW Version 3.9, released in April 2017, can be found in the modeling system user's guide by Wang et al. (2017).

A sensitivity test was completed to determine which options in WRF led to the most representative precipitation field for this particular event. Several combinations of options were experimented with, including dataset for initial conditions, simulation starttime, domain size, grid resolution, physics options, etc. The precipitation results (e.g., amount, location, etc.) from each simulation were compared to the observed event using the National Centers for Environmental Prediction (NCEP) Stage-IV 4-km gridded 
precipitation product (Lin and Mitchell 2005) in the Developmental Testbed Center (DTC) Model Evaluation Tools (MET) and the associated Method for Object-Based Diagnostic Evaluation (MODE). The statistical results from MODE were used to identify the simulation that best represented the precipitation pattern of the event.

\subsubsection{Model Specifications}

The WRF simulation with the closest representation of the precipitation field of the South Carolina flood was a 108-hour simulation initialized on 0000 UTC 01 October 2015 using a 280 x 280 horizontal grid with 15-km grid spacing, Lambert Conformal map projection centered at $32.0^{\circ} \mathrm{N}, 78.5^{\circ} \mathrm{W}$, and initial and lateral boundary conditions provided by $0.141^{\circ} \mathrm{x} \sim 0.141^{\circ}$ analysis data from the European Centre for Medium-Range Weather Forecasts (ECMWF). The model top was set to $50 \mathrm{hPa}$ with 50 customized vertical eta levels to provide higher vertical resolution within the planetary boundary layer (PBL). The parameterization schemes used are listed in Table 3.1 and were used in the control simulations of all 5 events. This simulation is referred to as the control simulation because no manipulations to the initial conditions were made.

For the South Carolina flood control simulation, an inner domain with 3-km grid spacing, domain 2, was nested within the 'parent' outer domain, hereafter referred to as domain 1 (Fig. 3.1). The inner domain consists of $321 \times 321$ horizontal grid points and covers South Carolina and North Carolina, the region which experienced the heaviest precipitation. Domain 2 uses the same vertical resolution and parameterization schemes 
Table 3.1. The parameterization schemes, and corresponding references, that created the most realistic control simulation of the South Carolina flood event of October 2015. These schemes were consistent throughout this research.

\begin{tabular}{l|l}
\hline \multicolumn{1}{c|}{ Parameterization Scheme } & \multicolumn{1}{c}{ Reference } \\
\hline WRF-single-moment (WSM) 6-class microphysics & Hong and Lim 2006 \\
\hline $\begin{array}{l}\text { Rapid Radiative Transfer Model-Global (RRTMG) } \\
\text { longwave and shortwave radiation }\end{array}$ & Iacono et al. 2008 \\
\hline Unified Noah Land-Surface Model & Tewari et al. 2004 \\
\hline Monin-Obukhov (MM5) similarity theory surface & $\begin{array}{l}\text { Dyer and Hicks 1970; Paulson } \\
\text { 1970: Webb 1970; Zhang and }\end{array}$ \\
\hline Yonsei University (YSU) PBL & Anthes 1982; Beljaars 1994 \\
\hline Tiedtke cumulus & Hong et al. 2006 \\
\hline
\end{tabular}

as domain 1 , with the exception of no cumulus parameterization scheme due to this domain's high horizontal resolution. Domain 2 is also initiated 6 hours later than domain 1 to allow for proper 'spin-up' time of domain 1 before it provides initial conditions to domain 2. Only one-way feedback between the domains is permitted to allow for the analysis of the simulation with and without domain 2, due to domain 1 having a more realistic representation of the strength and location of the cyclones, as well as the precipitation amount and location, than domain 2. See Appendix A for domain configurations for the remaining 4 events. 


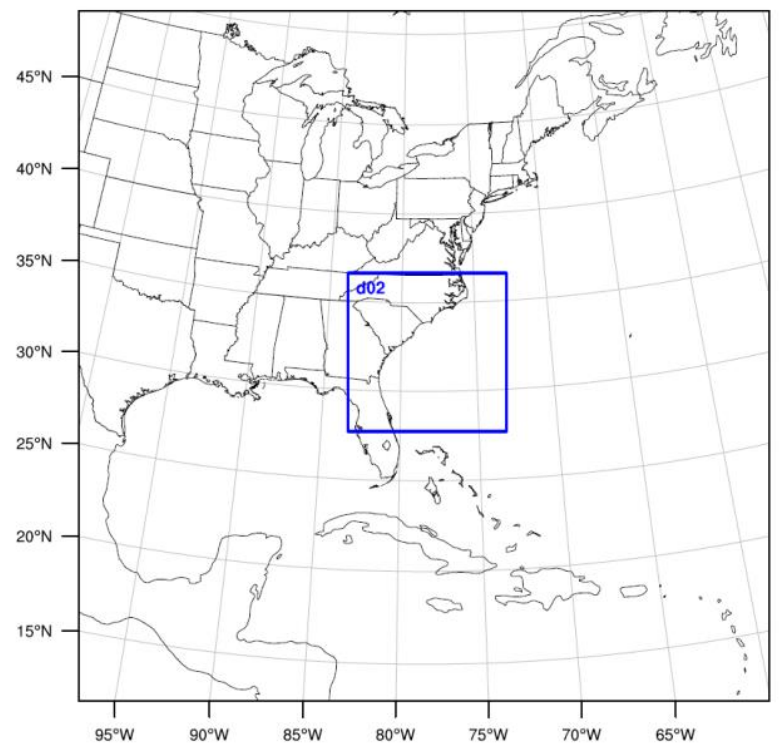

Figure 3.1. WRF model domains representing the outer domain (domain 1 ) of 15-km grid spacing with the inner domain (d02) of 3-km grid spacing outlined in blue.

\subsubsection{Experimental Simulations}

After performing the sensitivity test to establish the control simulation, an experimental simulation was completed in order to investigate the role of the tropical cyclone in the Joaquin and Katia events and contribute to Objective 2. A vortex removal tool exists in the WRF model to remove the vortex associated with a tropical cyclone from the model initial conditions and integration (Fredrick et al. 2009). This scheme allows for the removal of an existing vortex or the addition of a new vortex, and is referred to as the "TC bogus scheme" (Fredrick et al. 2009; Tang et al. 2013). The vortex removal process begins by searching for a vortex in a first guess field. The vortex is found by locating the maximum relative vorticity, assumed to be the center of the vortex, in the pressure field nearest the surface within a prescribed radial distance from observed 
location of the tropical cyclone (Fredrick et al. 2009). After locating the vortex, the scheme removes the first guess velocity and divergence within a $300 \mathrm{~km}$ radius of the first guess storm and then recalculates the velocity. Mathematically, the vorticity is calculated by:

$$
\nabla^{2} \psi=\zeta
$$

where $\psi$ is the streamfunction for the nondivergent wind and $\zeta$ is the relative vorticity. The vorticity is set to zero outside a radius of $300 \mathrm{~km}$ to define the nondivergent wind. The Dirichlet boundary conditions streamfunction is defined as zero and the Successive Over-Relaxation (SOR) method is used to solve equation (3.1) for the perturbation streamfunction on all pressure surfaces. The nondivergent wind $v_{\psi}$ is calculated from:

$$
v_{\psi}=\hat{k} \times \nabla \psi
$$

After the nondivergent wind is calculated, it is subtracted from the first guess wind fields. Removal of the divergent wind from the first guess storm is similar and described in detail in Fredrick et al. (2009). Further, the geopotential height and temperature anomalies are removed, and the surface and sea-level pressure perturbations associated with the height anomalies are removed from the surface and sea-level pressure fields. The final product is only background flow where the first guess storm was originally located (Fredrick et al. 2009).

Using this "TC bogus scheme" and following the methodology of Tang et al. (2013), WRF simulations for the Joaquin and Katia events were completed using the same options and domains as the respective control simulations, but with the removal of the tropical cyclone vortex, to help determine the role of the cyclone in the heavy 
precipitation event. In removing the vortex associated with the tropical cyclone, the following parameters were used: for the Joaquin event, a center location of Hurricane Joaquin at $0000 \mathrm{UTC} 01$ October 2015 of $23.9^{\circ} \mathrm{N}$ and $72.9^{\circ} \mathrm{W}$ according to the National Hurricane Center (NHC; Berg 2016), a maximum sustained wind speed of $51.4 \mathrm{~m} \mathrm{~s}^{-1}$, and a radius of maximum wind of $75 \mathrm{~km}$; for the Katia event, a center location of Hurricane Katia at $1200 \mathrm{UTC} 06$ September 2011 of $26.7^{\circ} \mathrm{N}$ and $65.6^{\circ} \mathrm{W}$ according to the NHC (Stewart 2012), a maximum sustained wind speed of $54.02 \mathrm{~m} \mathrm{~s}^{-1}$, and a radius of maximum wind of $100 \mathrm{~km}$.

Further investigation of each event through individual deterministic simulations using WRF was required to fully understand the cause of the extreme rainfall. Additional simulations were initialized at the same start times as the respective control simulations and run for the same amount of time with the same parameterization schemes. These simulations consisted of the removal of several environmental aspects, including terrain, latent heating, evaporation from precipitation, and surface heat fluxes, as well as the doubling of terrain height.

The removal of terrain was used to assess the importance of the frontal boundary in the event (Objective 2). It was hypothesized that removing the Appalachian Mountains from the model should change the simulated location and/or strength of the coastal front. Topography values in the WRF Preprocessing System (WPS) geographical input data were multiplied by 0.01 using a NCAR Command Language (NCL) script in order to minimize interactions with the atmosphere. The doubling of terrain may also impact the coastal front, but when compared to the simulation without terrain and the control simulation, it was used to assess the impact of the Appalachian Mountains on the event 
(Objective 2). The terrain was doubled in the WPS data using a NCL script similar to the aforementioned script.

Removing latent heating, surface heat fluxes, and evaporation from precipitation helped to determine the cause of the heavy precipitation through the identification of the role of each in the initialization and maintenance of convection throughout the event (Objective 3), as well as provide a means to analyze the impact of diabatic processes on the inhibited forward motion of the upper-level low and coastal front. Similar modifications to model simulations were made by Colle (2003) to study the evolution of Hurricane Floyd (1999) with a nearby frontal boundary. It was determined that latent heating was responsible for nearly two-thirds the strength of the surface temperature gradient along the East Coast and latent heating contributed to the strength and location of the 500-hPa trough. Evaporation from precipitation contributed to the strength of a low-level baroclinic zone and acted to lower the central pressure of Hurricane Floyd (1999), while surface heat fluxes were associated with the strength of deformation frontogenesis (Colle 2003). Experiments of this type help determine if diabatic effects enhanced the frontogenetical circulations near the coast in each event of this study, as was the case in Colle (2003).

Latent heating and surface heat fluxes were removed from simulation start to finish using namelist options in WRF. The removal of evaporation from precipitation required a slight (but simple) modification to the code of the WSM 6-class microphysics scheme before running the WRF model with no change to the namelist file. To maintain the atmospheric conditions required to initiate convection, evaporation was not removed until 6 hours before the start of the intense convection period for each event. 

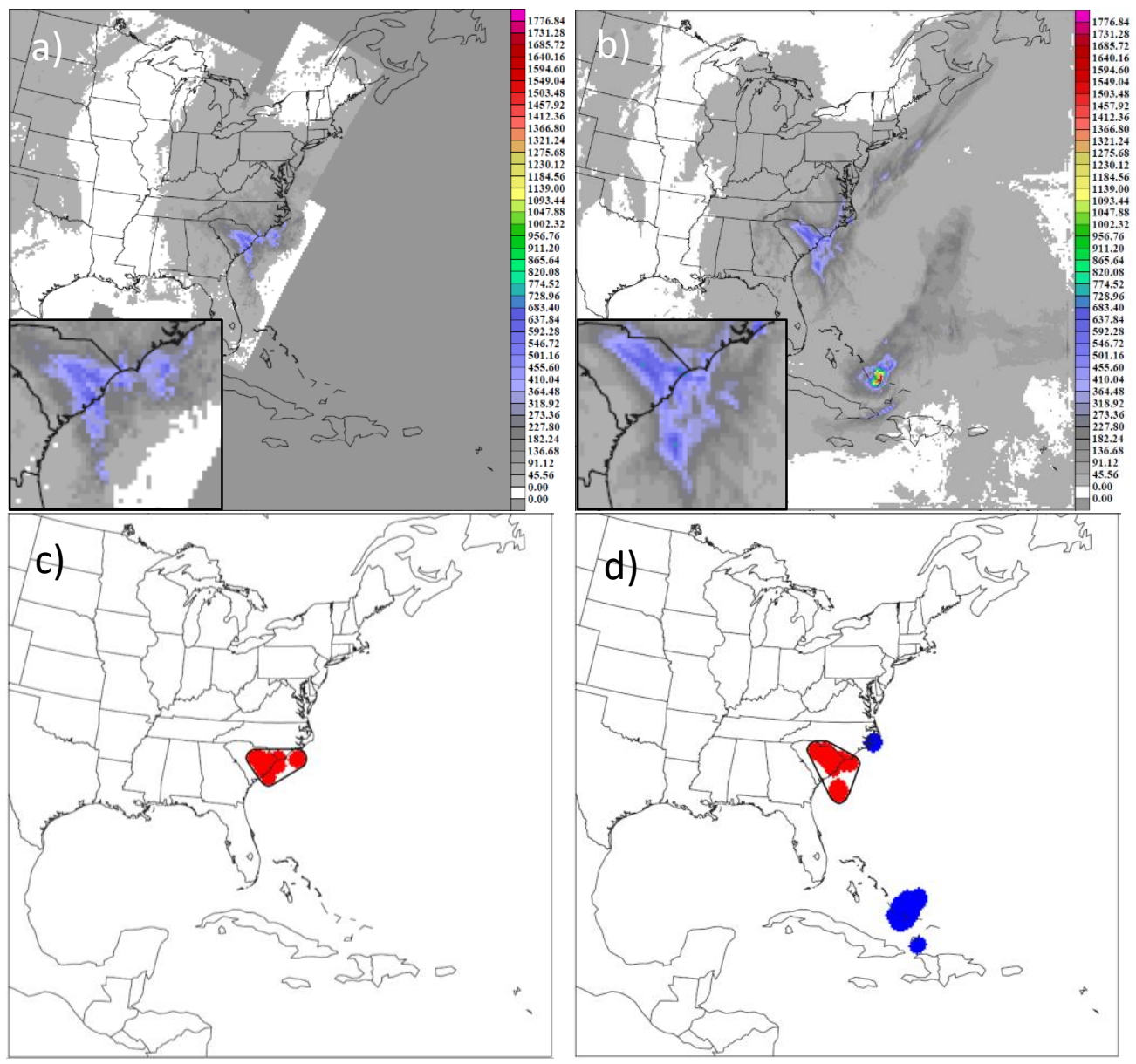

Figure 3.2. Precipitation amount ( $\mathrm{mm}$ ) for the South Carolina flood from 0000 UTC 01 October 2015 to 1200 UTC 05 October 2015 created using a) 4-km NCEP Stage IV reanalysis data from Earth Observing Laboratory (EOL) and b) WRF control simulation output for domain 1. The MODE results are shown to identify an object (red) for c) the reanalysis data shown in a) and d) the WRF data shown in b) that are corresponding in both analyses based on a threshold of $508 \mathrm{~mm}$. The total interest value calculated in MODE for the objects (red) shown in c) and d) is 0.96 .

\subsubsection{Model Verification}

Using MET and MODE, a total interest value was used to analyze the precipitation results, comparing several attributes (e.g., angle, ratio of area, edge separation, etc.) of two objects defined by a precipitation amount threshold (Davis et al. 


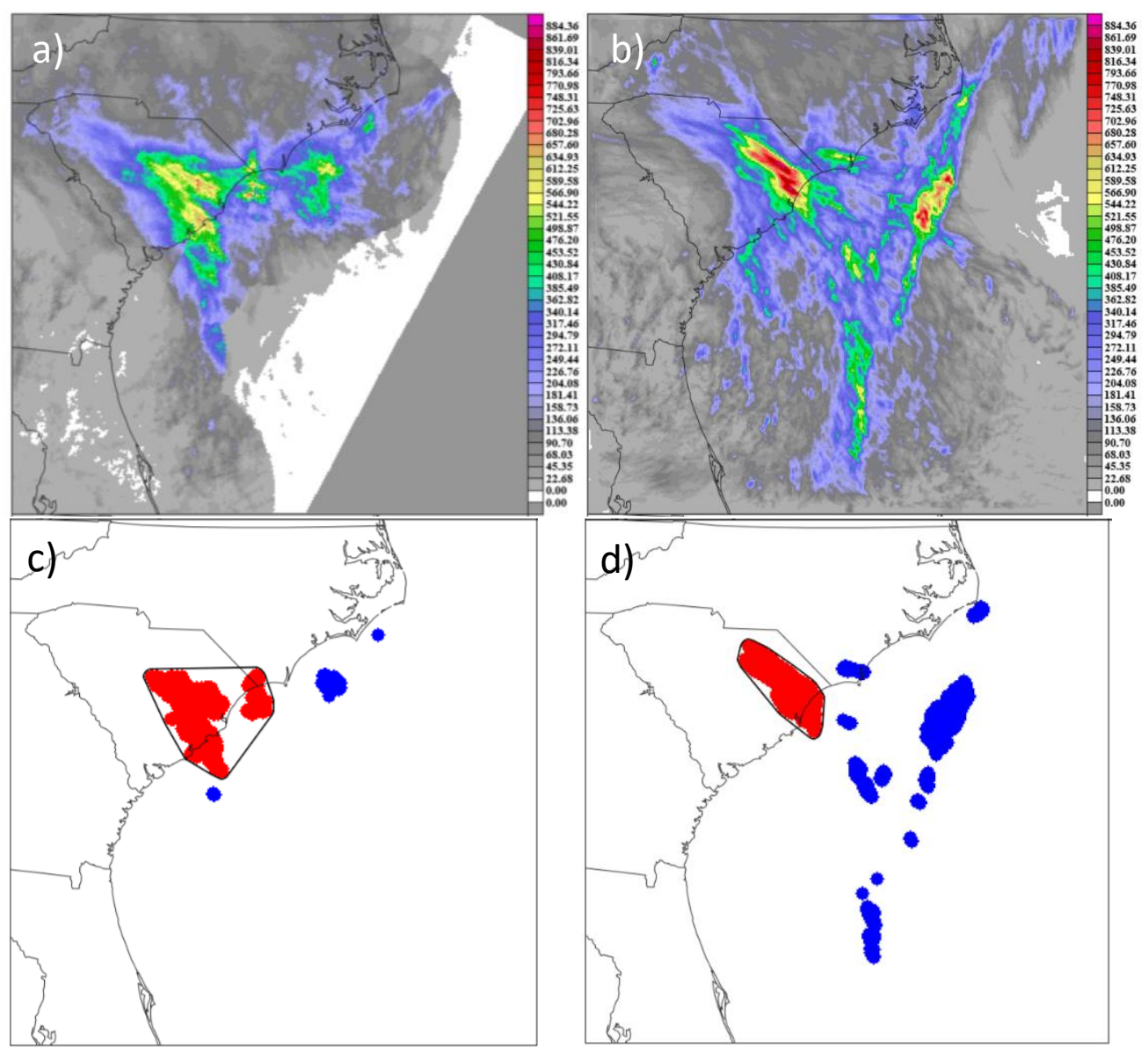

Figure 3.3. As in Fig. 3.2, except for domain 2 with a total interest value of 0.93 .

2009). Domain 1 (Fig. 3.2) had a total interest value of 0.96 (perfect representation being 1.0) and domain 2 (Fig. 3.3) had a total interest value of 0.93 at a threshold of $508 \mathrm{~mm}$ (20 inches) when compared to the NCEP Stage IV reanalysis of the actual event precipitation. The maximum accumulated precipitation over the 108-hour period from 0000 UTC 01 October to 1200 UTC 05 October 2015 was $682.75 \mathrm{~mm}$ (26.88 in.) in Mount Pleasant, SC. The maximum for domain 2 of the WRF reconstruction was 907.04 $\mathrm{mm}$ (37.71 in.) located $30 \mathrm{~km}$ southeast of Florence, SC or about $145 \mathrm{~km}$ north of Mount 

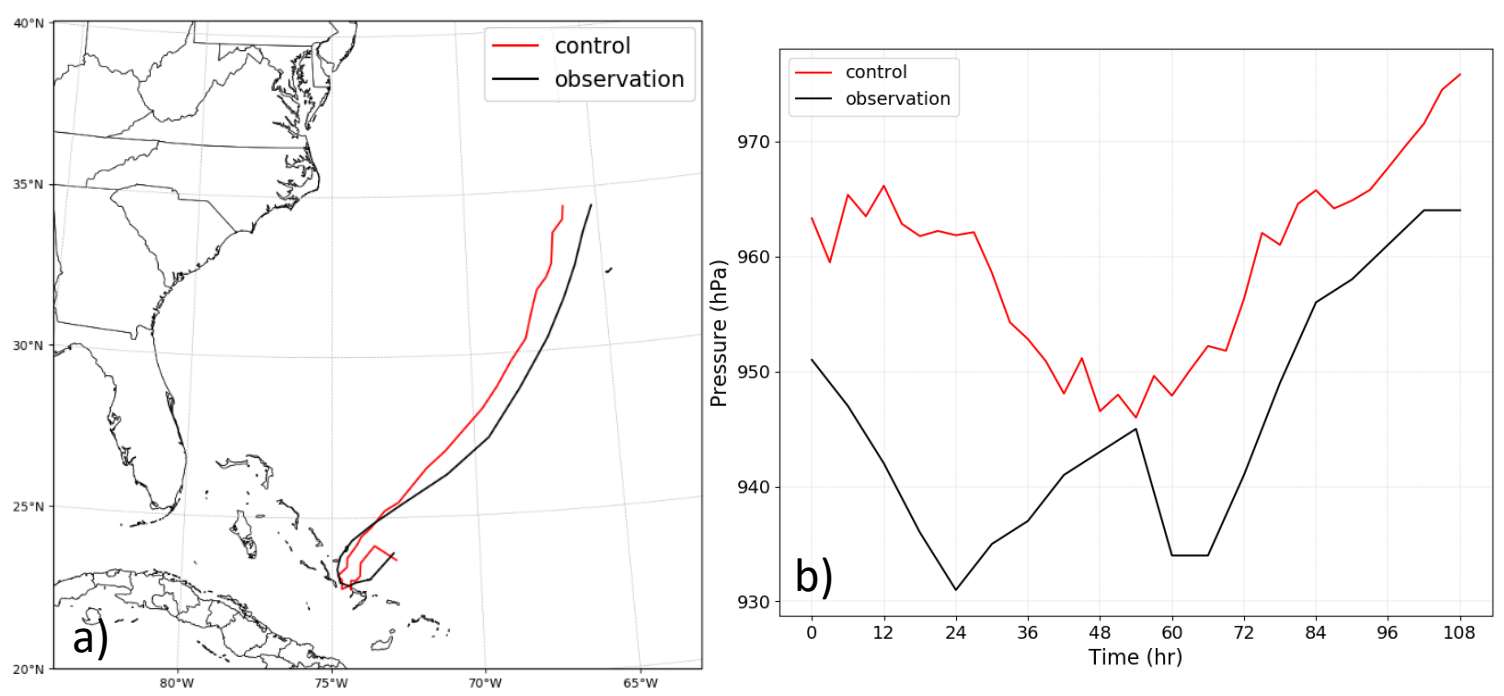

Figure 3.4. Hurricane Joaquin a) tracks and b) central pressures from 0000 UTC 01 October 2015 to 1200 UTC 05 October 2015 resulting from the National Hurricane Center best track analysis (black) and WRF d01 control simulation (red).

Pleasant, SC. Even though the maximum precipitation location was displaced by WRF, the axis of heaviest precipitation (Fig. 3.3b) nearly aligns with the northernmost axis of heavy rain from the NCEP Stage IV reanalysis (Fig. 3.3a).

With Hurricane Joaquin's impact on the heavy precipitation in question, it was important to assess WRF's ability to capture the tropical cyclone track and intensity. Figure 3.4a represents the best track of Hurricane Joaquin (Berg 2016; black), along with the cyclone track resulting from domain 1 of the WRF control simulation (red). Figure 3.4b represents similar results as Fig. 3.4a, except showing the central pressure of the tropical cyclone for the duration of the simulations. The WRF control simulation was able to capture the early southwesterly movement of Hurricane Joaquin, along with the northerly turn and resulting track towards Bermuda (Fig. 3.4a). The location of the tropical cyclone at 0000 UTC 01 October 2015 in the control simulation did not match 
the best track position, likely due to the ECMWF initial conditions passed to WRF being slightly inaccurate. WRF did struggle with the intensity of Hurricane Joaquin. The best track analysis shows Joaquin undergoing two periods of rapid intensification, while WRF has the cyclone gradually intensifying through hour 60 and then gradually weakening for the remainder of the simulation (Fig. 3.4b). While WRF did not produce the exact tropical cyclone track and strength as the best track analysis, the precipitation results are sufficient for this research.

\subsection{Data Analysis}

All aforementioned WRF experiments for the 2015 South Carolina flood event were repeated for the heavy rainfall event involving Hurricane Katia in 2011. Both events were thoroughly examined through each experimental simulation to obtain a synoptic, mesoscale, and local scale analysis. Additionally, control and experimental simulations were completed using WRF for the three events without the presence of an offshore tropical cyclone. These simulations were used to compare synoptic and mesoscale environments of events with offshore tropical cyclones to those without (Objectives 1 and 2), as well as to determine the impacts of topography, latent heating, surface heat fluxes, and evaporation from precipitation on extreme precipitation events that are not influenced by a tropical cyclone. All five events occur along the East Coast and involve an extratropical cyclone, a coastal front, and a 24-hour total precipitation amount greater than $250 \mathrm{~mm}$; all were analyzed for the cause and type of convection. 


\subsubsection{Synoptic Scale}

A synoptic scale analysis was necessary to investigate the large-scale contribution to the heavy precipitation during the events (Objective 1). The interaction between the tropical and extratropical cyclones, as well as the cyclone interactions with the coastal front, were important to investigate for the Joaquin and Katia cases. The horizontal resolution of the WRF output was too high and too noisy to show irrotational wind, so a quick analysis of the synoptic situation during the Joaquin event was completed with a plot of 300-200-hPa layer-averaged potential vorticity (PV) and irrotational wind, along with $250-\mathrm{hPa}$ wind and 600-400-hPa ascent, using the $0.5^{\circ}$ NCEP Climate Forecast System Reanalysis (CFSR) gridded dataset (Saha et al. 2014) at a time of heavy precipitation for South Carolina. Together, those 4 variables provided an understanding of what atmospheric conditions were coming together to produce such extreme rainfall and prolonged convection.

A variety of plan-view analyses were used to compare the interactions in each event in terms of synoptic environments and dynamics (e.g., cyclone strengths, distance between cyclones, jet streams, moisture availability, etc.). The majority of variables plotted were pulled directly from WRF output files or calculated using software such as Read/Interpolate/Plot (RIP), WRF-Python, or MetPy. An exception was horizontal water vapor flux, known as integrated vapor transport (IVT). A Python script was created to calculate specific humidity (Bolton 1980) and then IVT using the sum of 50-hPa layer averages from $1050 \mathrm{hPa}$ to $250 \mathrm{hPa}$, following the method of Mueller et al. (2017),

$$
I V T=\frac{1}{g} \int_{1050 h P a}^{250 h P a} q * U * d p
$$




\subsubsection{Mesoscale}

Frontogenesis and forcing for ascent are important characteristics in events of this magnitude involving frontal boundaries. To quantify the forcing needed to create vertical circulations and the change in horizontal temperature gradients, Miller (1948) frontogenesis was calculated on pressure levels. Miller (1948) frontogenesis is the Lagrangian rate of change of the magnitude of the three-dimensional gradient of potential temperature due to the three-dimensional wind, an extension of the two-dimensional Petterssen (1936) frontogenesis equation (Schultz and Doswell 1999). The Miller (1948) frontogenesis equation can be written as:

$$
\begin{aligned}
F=\frac{d}{d t}\left|\nabla_{h} \theta\right|=-\frac{1}{\left|\nabla_{h} \theta\right|}\{ & {\left[\left(\frac{\partial \theta}{\partial x}\right)^{2}\left(\frac{\partial u}{\partial x}\right)+\left(\frac{\partial \theta}{\partial y}\right)^{2}\left(\frac{\partial v}{\partial y}\right)\right]+\left[\frac{\partial \theta \partial \theta}{\partial x \partial y}\left(\frac{\partial v}{\partial x}+\frac{\partial u}{\partial y}\right)\right]+\left[\frac{\partial \theta}{\partial z}\left(\frac{\partial w}{\partial x} \frac{\partial \theta}{\partial x}+\frac{\partial w}{\partial y} \frac{\partial \theta}{\partial y}\right)\right] } \\
\mathrm{A} & \left.-\left[\frac{\partial \theta}{\partial x} \frac{\partial}{\partial x}\left(\frac{d \theta}{d t}\right)+\frac{\partial \theta}{\partial y} \frac{\partial}{\partial y}\left(\frac{d \theta}{d t}\right)\right]\right\}
\end{aligned}
$$

where term $\mathrm{A}$ is the horizontal deformation and divergence, important for driving ageostrophic direct circulations, term $\mathrm{B}$ is the tilting term, and term $\mathrm{C}$ represents the differential diabatic processes. Terms B and $\mathrm{C}$ account for vertical circulations that may impact the horizontal temperature gradient (Colle 2003). The components of (3.5) were calculated to determine the dominant term in each event examined. Horizontal deformation has been found to be an important mechanism in the creation and maintenance of frontogenesis at the surface and in the upper levels (Mudrick 1974). Therefore, horizontal deformation was analyzed closely for each case to determine if it 
was working to tighten the buoyancy gradient and support a positive feedback system needed for the development and maintenance of a strong stationary front (Objective 2).

\subsubsection{Parcel Trajectories}

On a small scale, the parcel environment was analyzed as it crossed the frontal boundary using the 3-km WRF simulation data. The Read/Interpolate/Plot (RIP) program provided the ability to obtain diagnostic variables along trajectories (Stoelinga 2009). Using RIP, cross sections and thermodynamic profiles were created along the parcel trajectory to allow for the assessment of the dominant forcing mechanism in the region of convective initiation. The coastal front for each event was closely analyzed to determine its strength and contribution to the heavy precipitation (Objective 2), while the convection was investigated and defined as elevated or surface-based (Objective 3). 


\section{CHAPTER 4. RESULTS}

This research began by investigating the cause of the 2015 South Carolina flood. After obtaining an understanding of the event and the atmospheric features that contributed to the intense precipitation, the role of each atmospheric feature and process were analyzed using the results from the WRF experimental simulations of the event. The same process was completed for each event, but only the comparison of the additional events to the 2015 South Carolina flood were documented.

\subsection{South Carolina Flood}

As discussed earlier (section 2.4.1.4), the most intense rainfall occurred between 1200 UTC 03 October and 1200 UTC 04 October 2015 in Mount Pleasant, South Carolina during the flood event. The WRF control simulation of the event was consistent with the timing of the rainfall (Fig. 4.1a), but the location of the heaviest rainfall was roughly 125 km north-northeast of Mount Pleasant, near Florence, South Carolina (Fig.

4.1b). The location difference was not an issue for this research due to the comparison of the WRF experimental simulations to the WRF control simulation, not the observed event. The analyses of the event focused on the hours between 1200 UTC 03 October and 1200 UTC 04 October 2015, which were accurately represented by WRF forecast hours 60 to 84 (Fig. 4.1a), as the purpose of this research was to investigate the intense precipitation. 

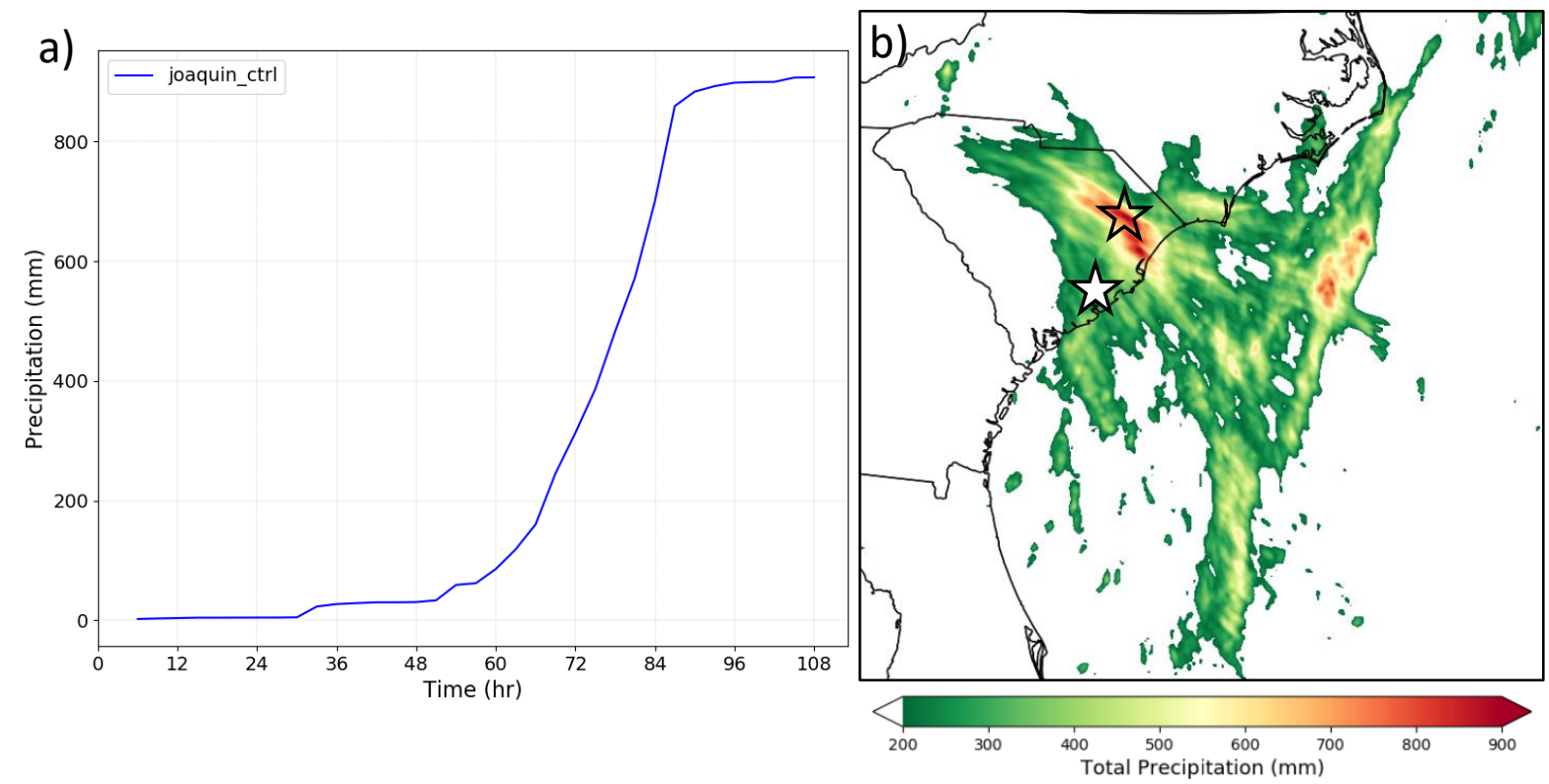

Figure 4.1. Precipitation ( $\mathrm{mm}$ ) for the South Carolina flood event from 0000 UTC 01 October 2015 to 1200 UTC 05 October 2015 for domain 2 of the WRF control simulation a) at the location of maximum precipitation, plotted as accumulation with time and b) as the event total for the region, with the white star (south) representing Mount Pleasant, South Carolina and the transparent star (north) representing the location of heaviest rainfall near Florence, South Carolina.

\subsubsection{Synoptic Scale}

Using the $0.5^{\circ}$ NCEP CFSR, several atmospheric features were identified at 1200 UTC 03 October 2015 over the southeastern United States and the western Atlantic Ocean (Fig. 4.2a). The negatively tilted, upper-level trough over the southeastern United States was associated with a region of higher PV, as well as a split upper-level jet oriented southeast-northwest over Louisiana and the Ohio River Valley. The location of the trough positioned South Carolina in the right entrance region of the upper-level jet streak, allowing for stronger mid-level ascent across the state from 1200 UTC 03 October (Fig. 4.2a) through 0600 UTC 04 October 2015 (Fig. 4.2b). 


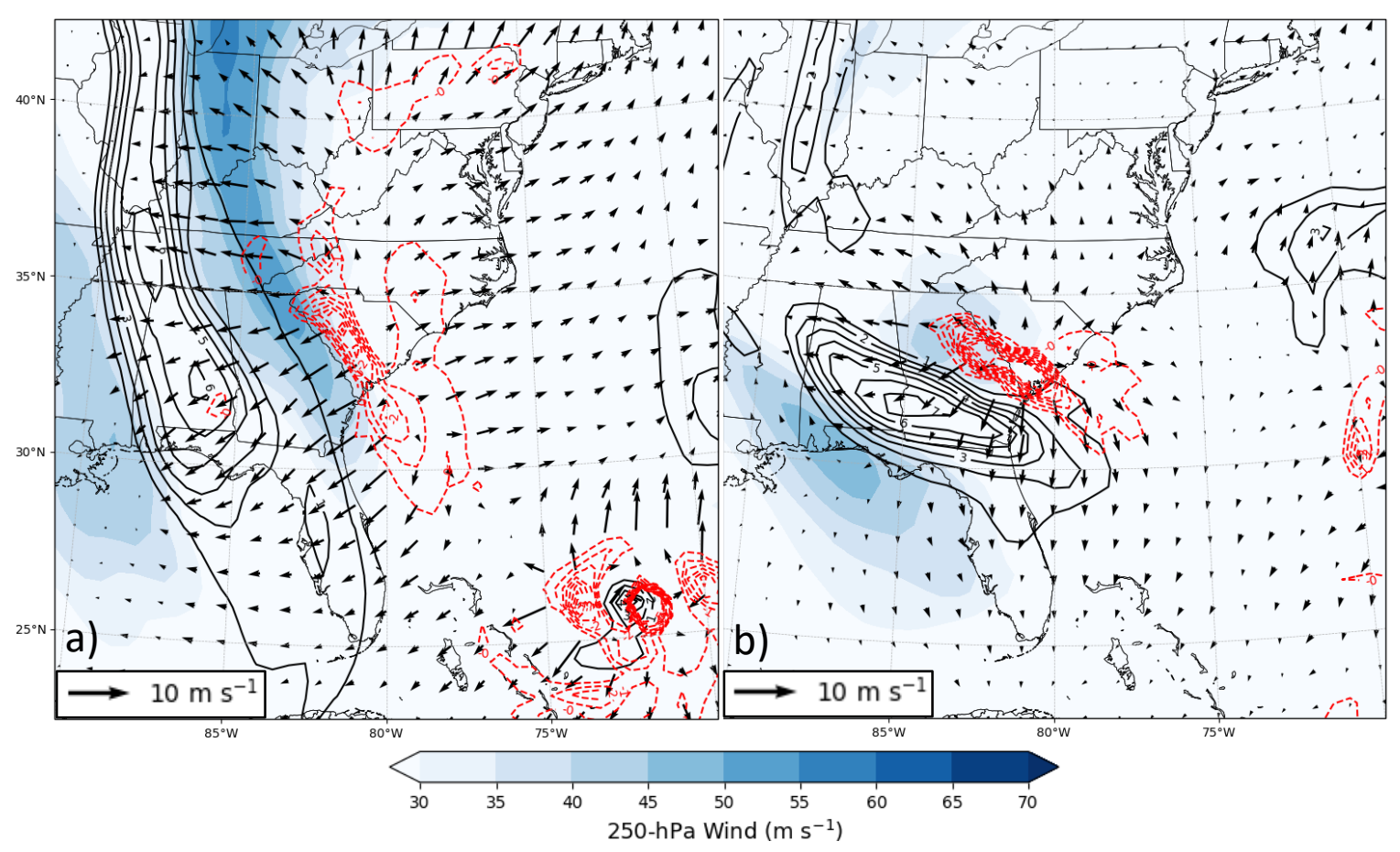

Figure 4.2. Climate Forecast System Reanalysis (CFSR) valid at a) 1200 UTC 03 October 2015 and b) 0600 UTC 04 October 2015 representing 250-hPa wind ( $\mathrm{m} \mathrm{s}^{-1}$; shaded), 600-400-hPa ascent $\left(5 \times 10^{-3} \mathrm{hPa} \mathrm{s}^{-1}\right.$; dashed red), 300-200-hPa potential vorticity (PVU; contoured) and irrotational wind ( $\mathrm{m} \mathrm{s}^{-1}$ ).

The area of increased mid-level ascent (Fig. 4.2a) was collocated with convection over South Carolina at 1200 UTC 03 October 2015 (Fig. 2.6a). Large amounts of latent heat release within the region of convection led to increased heights downstream of the upper-level trough and negative PV advection by the irrotational wind (Fig. 4.2a, b), which slowed the northeastward propagation of the trough (Torn 2017). The WRF simulation showed that the upper-level low remained southwest of South Carolina from 1200 UTC 03 October (Fig. 4.3a) through 1200 UTC 04 October 2015 (Fig. 4.3b), keeping South Carolina in the right entrance region of the upper-level jet with associated upper-level divergence (Fig. 4.3a, b). The feedback from convection inhibited northeastward motion and led to the upper-level trough becoming a cut-off low on 


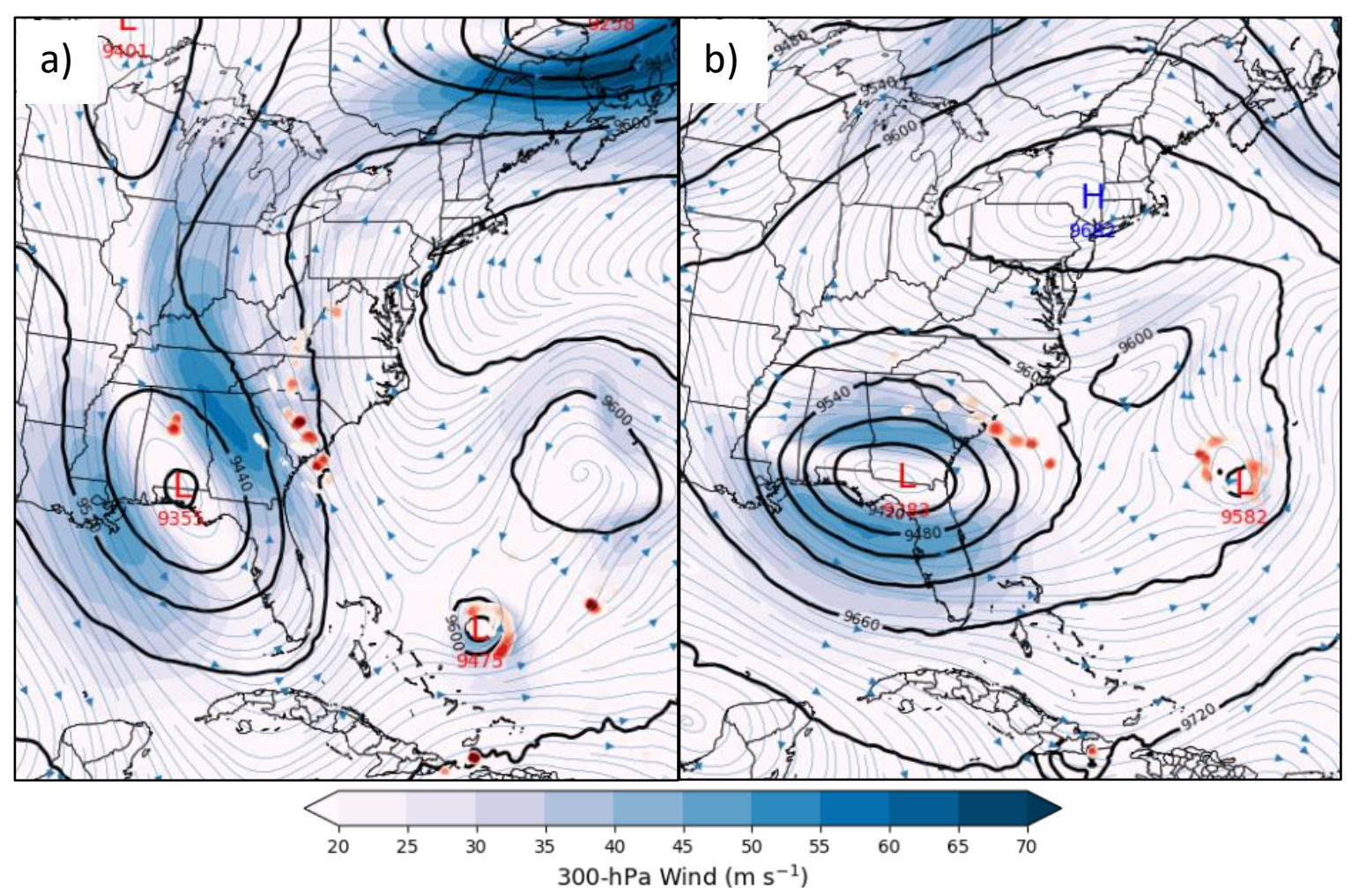

Figure 4.3. WRF control simulation 300-hPa height (gpm; thick contoured), wind ( $\mathrm{m} \mathrm{s}^{-1}$; shaded; thin streamlines), and divergence ( $10^{-5} \mathrm{~s}^{-1}$; contoured; positive red) at a) 1200 UTC 03 October 2015 and b) 1200 UTC 04 October 2015 for domain 1.

04 October 2015 (Fig. 4.3b). The upper-level jet streak associated with the low began to tilt into an east-west orientation across the southeastern United States (Fig. 4.3b) as the low propagated east, south of the convection. The eastward movement of the low became possible when Hurricane Joaquin finally began to move northeast due to the weakening ridge of high pressure off the coast of New England (Fig. 4.3b). The northern jet streak associated with the low eventually wrapped around the west side of the low and joined with the southern jet streak as the low pushed northeast on 05 October 2015, following Hurricane Joaquin into the northern Atlantic (not shown). 


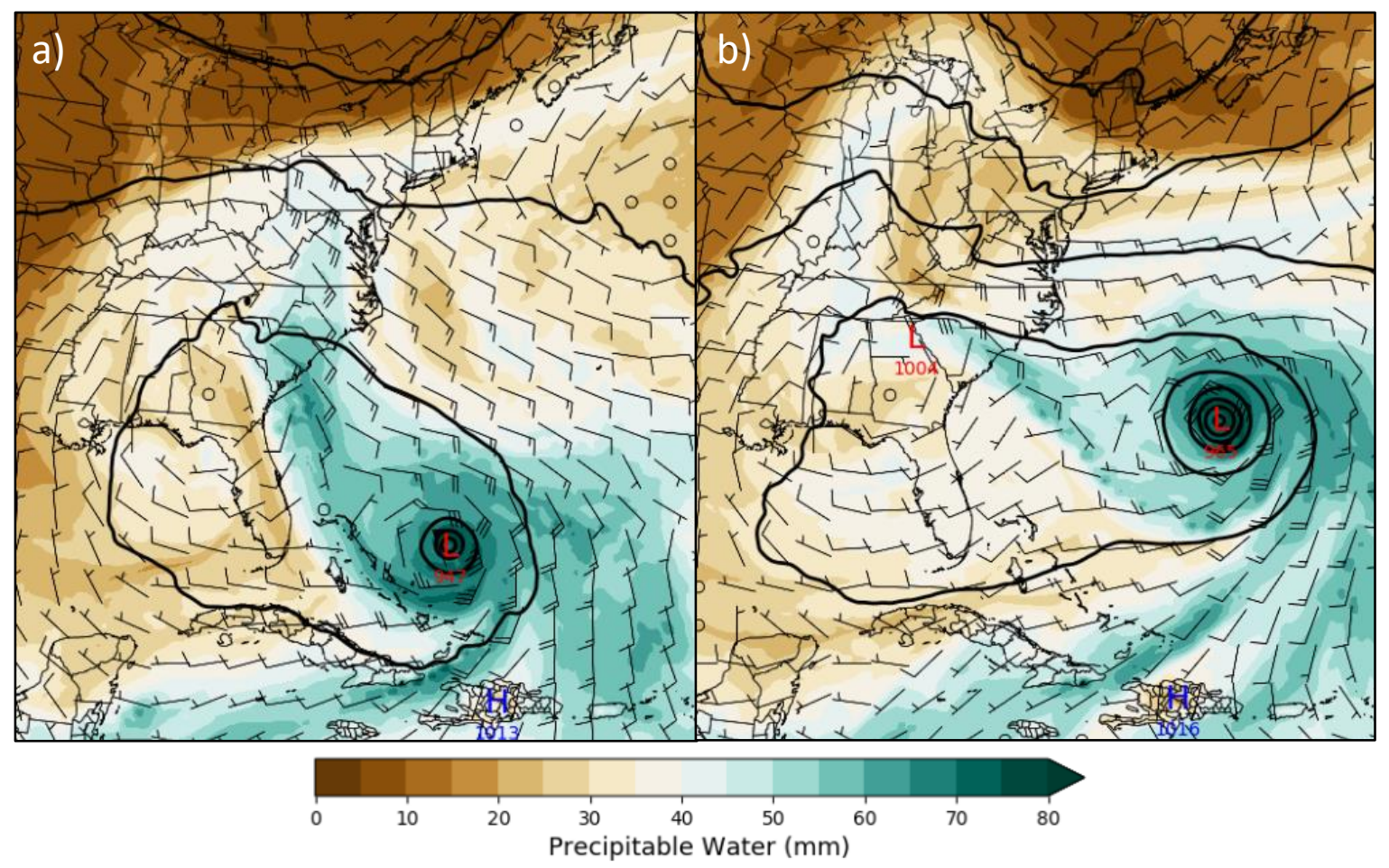

Figure 4.4. WRF results for domain 1 of the control simulation at a) 1200 UTC 03 October 2015 and b) 1200 UTC 04 October 2015 displaying precipitable water (mm; shaded), sea level pressure ( $\mathrm{hPa}$; contoured), and 850-hPa wind $\left(\mathrm{m} \mathrm{s}^{-1}\right)$.

Both WRF (Fig. 4.4a) and the NCEP CFSR (Fig. 4.2a) displayed plentiful moisture for the region, with precipitable water values near $60 \mathrm{~mm}$ stretching from South Carolina southeast to Hurricane Joaquin near the Bahamas at 1200 UTC 03 October 2015. Ample moisture was available to the South Carolina region for the 24-hour period, but by 1200 UTC 04 October 2015 precipitable water values began to slowly decrease below $50 \mathrm{~mm}$ (Fig. 4.4b). The decrease in moisture caused convection to weaken, which eventually allowed the cut-off low to propagate northeast.

To investigate the reason for the decrease in moisture, integrated water vapor transport (IVT) was analyzed for the region to determine where the moisture was coming 


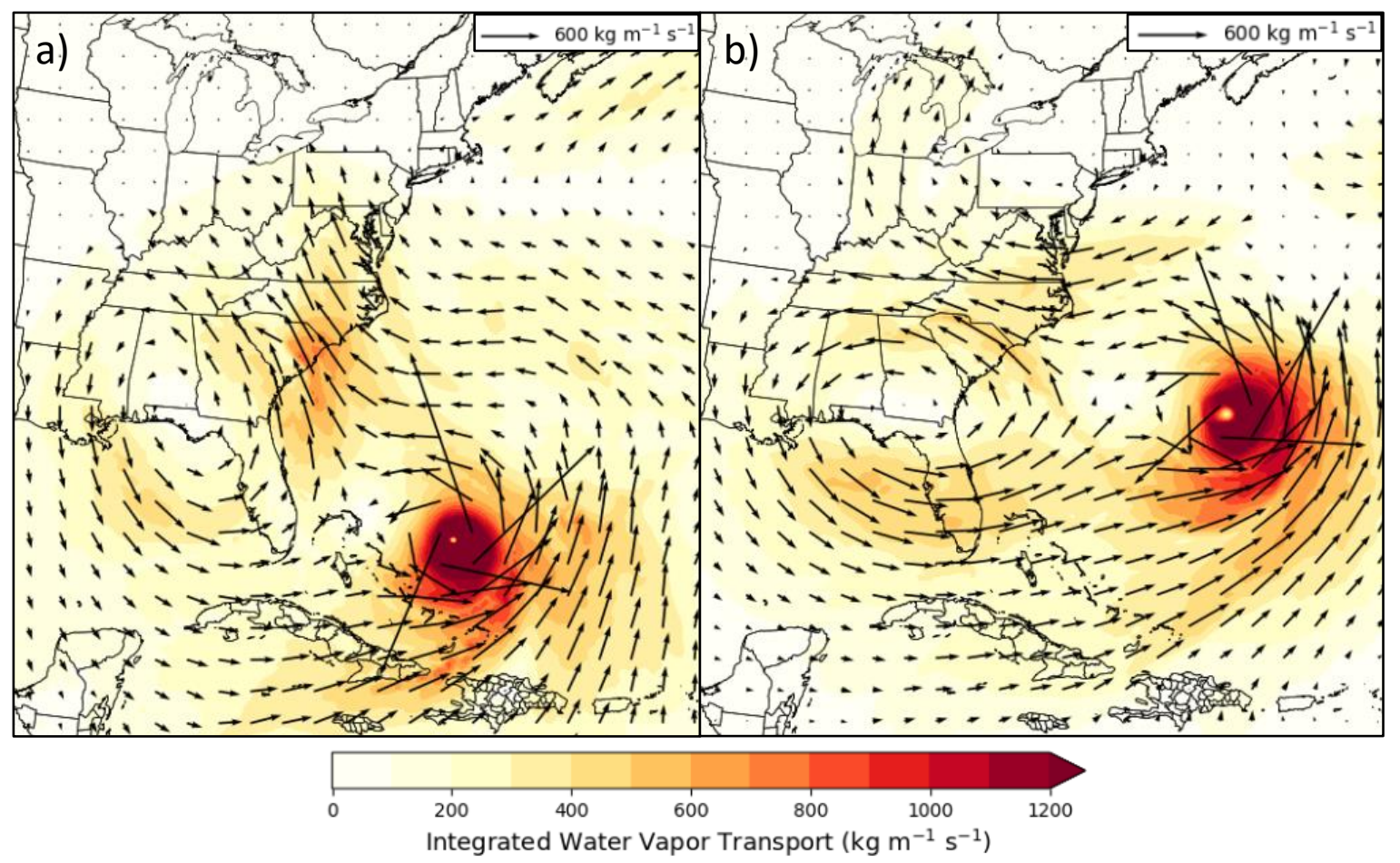

Figure 4.5. WRF results for domain 1 of the control simulation at a) 1200 UTC 03 October 2015 and b) 1200 UTC 04 October 2015 displaying integrated water vapor transport (IVT; $\mathrm{kg} \mathrm{m}^{-1} \mathrm{~s}^{-1}$ ).

from. IVT values approached $600 \mathrm{~kg} \mathrm{~m}^{-1} \mathrm{~s}^{-1}$ over coastal South Carolina at 1200 UTC 03

October 2015 (Fig. 4.5a), which qualified as an atmospheric river for the southeastern United States per Mahoney et al. (2016). This atmospheric river was located along the axis of dilatation and did not stretch far enough southeast to establish a clear connection to Hurricane Joaquin (Fig. 4.5a). However, the tropical cyclone may have contributed to the moisture through transport and outflow from the northwest quadrant, with IVT values over $1200 \mathrm{~kg} \mathrm{~m}^{-1} \mathrm{~s}^{-1}$ directed northwest (Fig. 4.5a). The upper-level low over the southeastern United States also contributed to the moisture transport by aiding the southeasterly flow into South Carolina, possibly pulling moisture from just off the coast of the southeastern United States along the Gulf Stream. The convergent flow along the 
axis of dilation between the two cyclones seemed to be the main reason for the increased moisture over South Carolina (Fig. 4.5a, b), drawing all available water vapor from the southeastern United States coast into a confined region from roughly 0000 UTC 03 October to 0000 UTC 05 October 2015.

Precipitable water values decreased over South Carolina (Fig. 4.4b) as Hurricane Joaquin moved northeast. The IVT pattern showed decreased transport values $(\sim 600 \mathrm{~kg}$ $\mathrm{m}^{-1} \mathrm{~s}^{-1}$ ) pointing towards South Carolina from Hurricane Joaquin at 1200 UTC 04 October 2015 (Fig. 4.5b). The decreased IVT from Hurricane Joaquin suggests that precipitable water values decreased over South Carolina because Hurricane Joaquin became positioned far enough north to no longer contribute as much moisture to the South Carolina region (Fig. 4.5b). Thus, the convection over South Carolina weakened from 04 October to 05 October 2015 as Hurricane Joaquin began to move northeast, away from the United States. This implied that Hurricane Joaquin played a significant role in the flood event, purely because the cyclone's northwestward outflow contributed additional moisture to the western Atlantic that could be transported into South Carolina along the axis of dilatation and upper-level jet associated with the cut-off low, allowing convection to continue for over 24 hours.

\subsubsection{Mesoscale}

While the synoptic scale supported convection through jet dynamics and significant moisture transport, the lift required to initiate convection was most likely the product of a mesoscale process (Doswell 1987). The rate of precipitation began to 


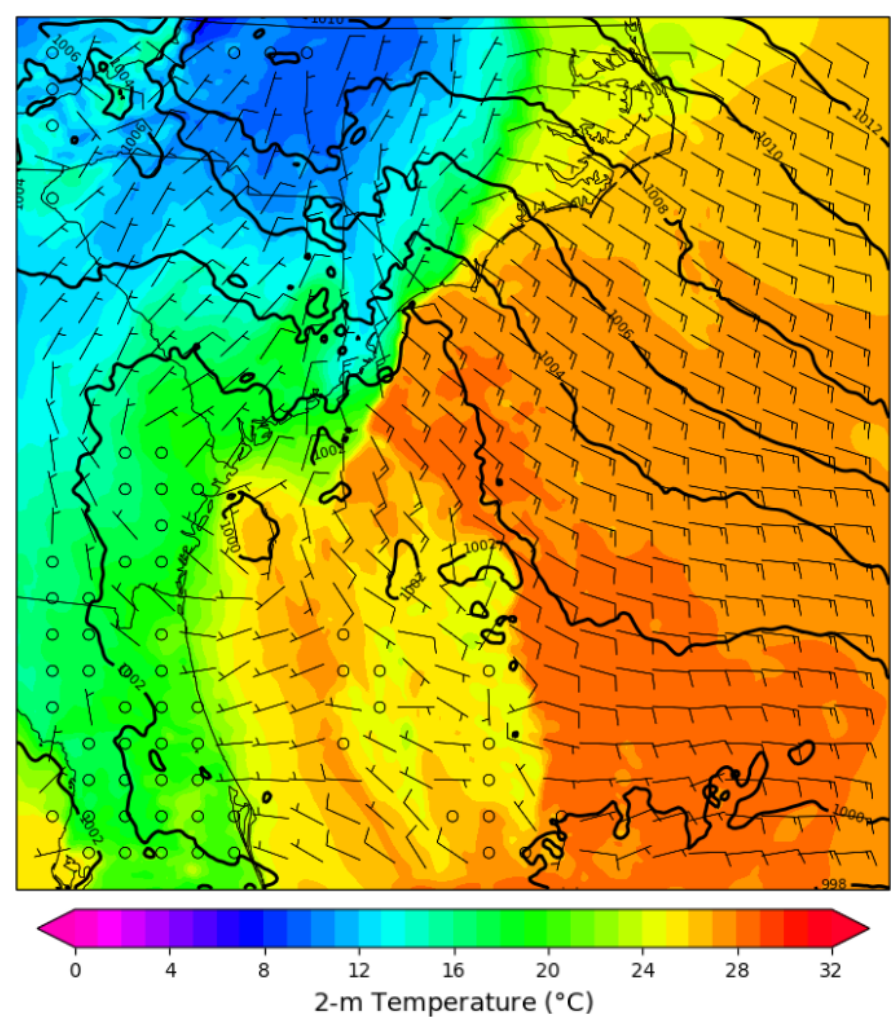

Figure 4.6. WRF results for domain 2 of the control simulation at 1200 UTC 03 October 2015 displaying sea level pressure ( $\mathrm{hPa}$; contoured), $2-\mathrm{m}$ temperature $\left({ }^{\circ} \mathrm{C}\right.$; shaded), and $10-\mathrm{m}$ wind $\left(\mathrm{m} \mathrm{s}^{-1}\right)$.

increase around 1200 UTC 03 October 2015 (Fig. 4.1a). An analysis of surface conditions for the mid-Atlantic region at that time of convective initiation showed a shift in surface winds from northerly over land to southeasterly just off the coast of South Carolina (Fig. 4.6). The wind shift combined with a sharp temperature gradient $\left(15-20^{\circ} \mathrm{C}\right.$ across $100 \mathrm{~km}$ ) showed the presence of a frontal boundary along the coast of South Carolina and North Carolina. The coastal front formed as a result of the upper-level low over the southeastern United States (Fig. 4.3a), in combination with the surface low off the coast of Georgia (Fig. 4.6), providing strong southeasterly flow into the region, while 


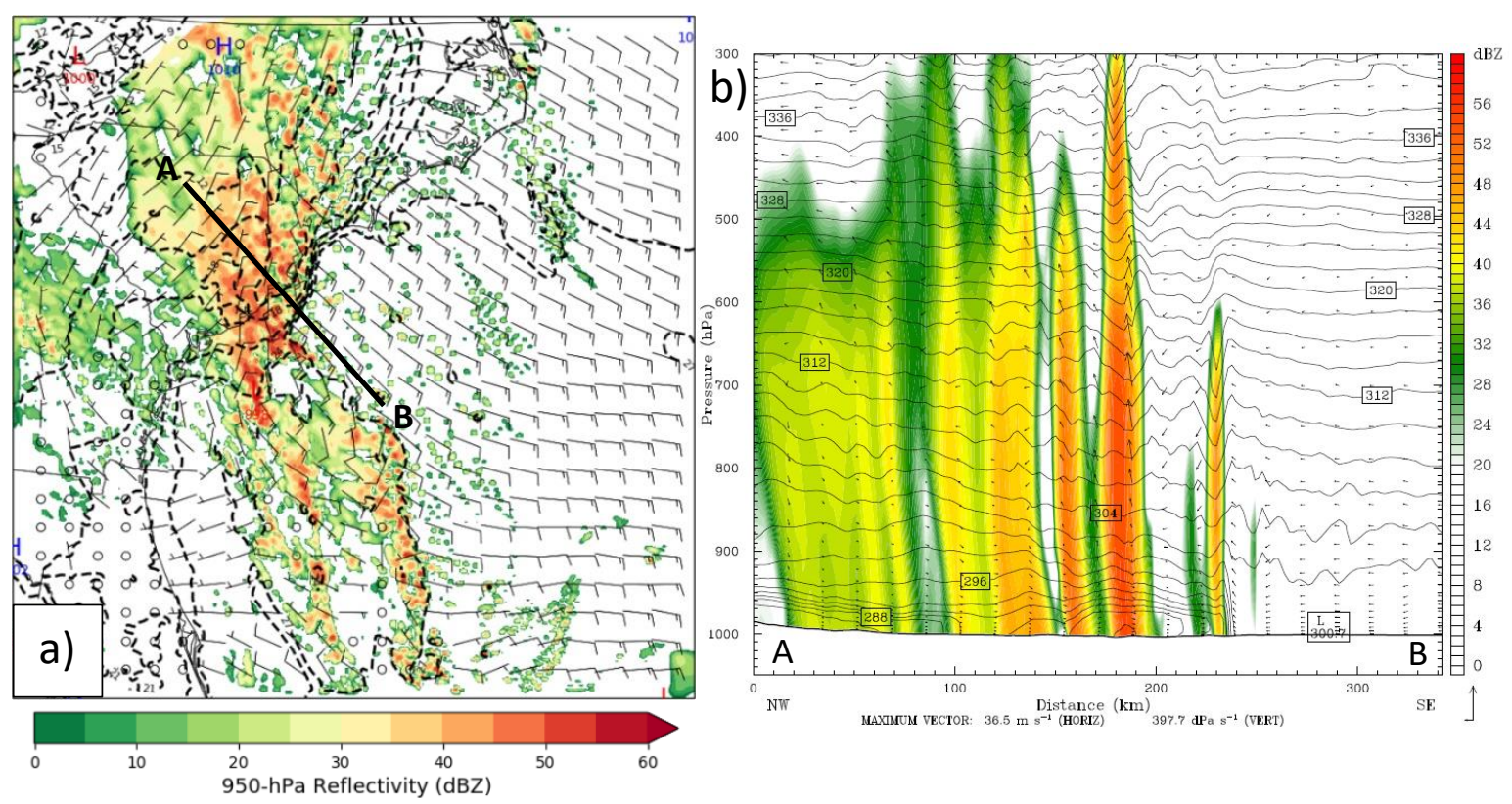

Figure 4.7. WRF results for domain 2 of the control simulation at 1200 UTC 03 October 2015 displaying a) 2-m temperature $\left({ }^{\circ} \mathrm{C}\right.$; dashed), 950-hPa reflectivity (dBZ; shaded), 10-m wind ( $\left.\mathrm{m} \mathrm{s}^{-1}\right)$ and b) potential temperature ( $\mathrm{K}$; contoured), reflectivity ( $\mathrm{dBZ}$; shaded), and circulation vectors $\left(\mathrm{m} \mathrm{s}^{-1}, \mathrm{dPa} \mathrm{s}^{-1}\right)$. Line segment $A B$ in a) represents the location of $b$ ).

the Appalachian Mountains allowed for the containment of cold air from the north (Fig. 4.6), known as cold air damming (Richwien 1980; Stauffer and Warner 1987). The coastal front and region of cold air damming were also identified by the inverted trough in sea level pressure along and east of the mid-Atlantic coast stretching south to an area of low pressure off the coast of Georgia with an adjacent inverted ridge just east of the Appalachians, respectively (Fig. 4.6).

Simulated reflectivity from WRF suggested the coastal front to be the source of lift for the convection. A plan-view analysis of 950-hPa reflectivity and 2-m temperature showed the majority of rainfall located west of the frontal boundary at 1200 UTC 03 October 2015 (Fig. 4.7a). A cross-sectional analysis supported the frontal boundary 


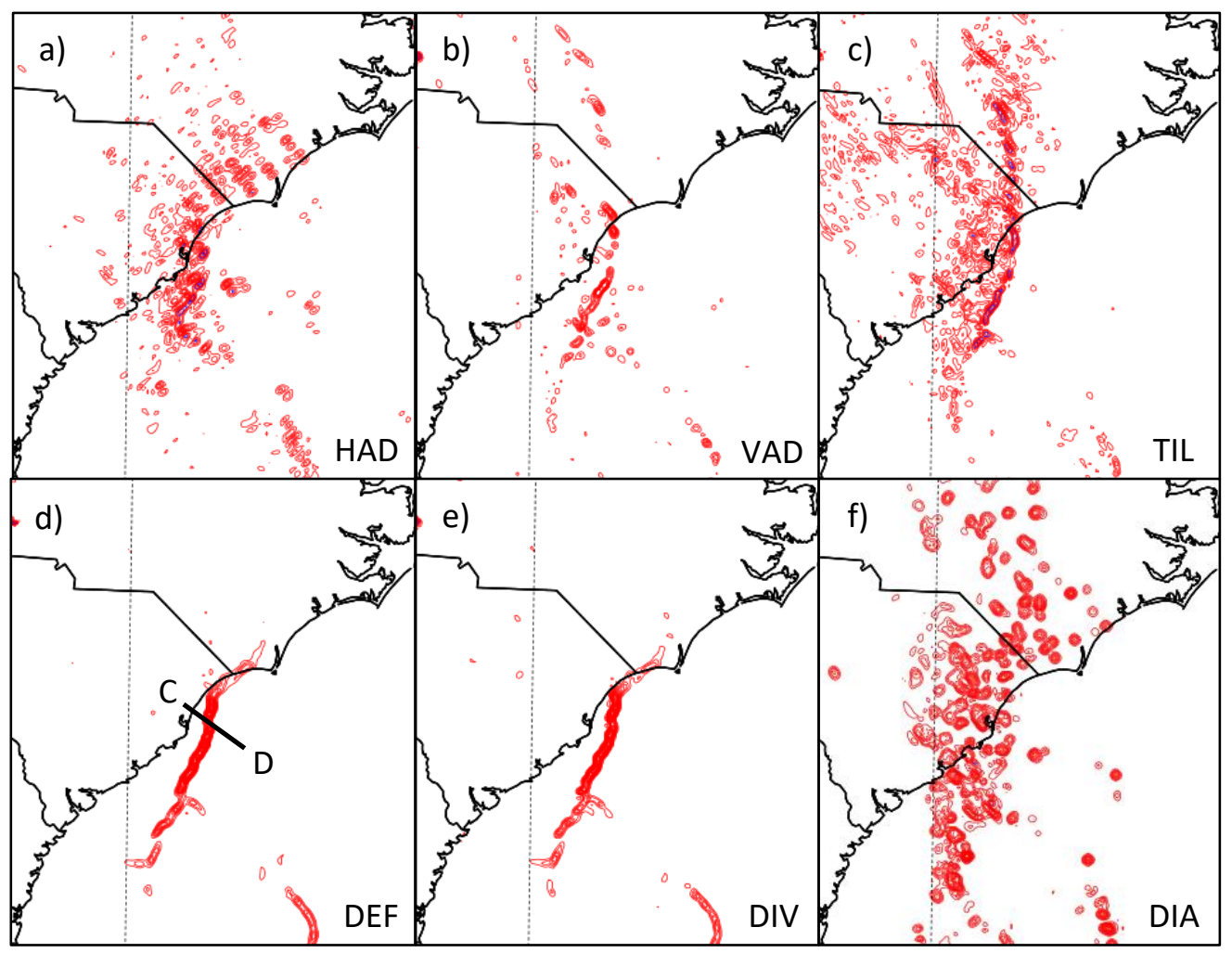

Figure 4.8. WRF domain 2 control simulation at 1200 UTC 03 October 2015 representing a) 700-hPa horizontal advective, b) 900-hPa vertical advective, c) 950-hPa tilting, d) 1000-hPa deformation, e) 1000 $\mathrm{hPa}$ divergence, and f) 700-hPa diabatic frontogenesis (positive red, negative blue) contoured every $50 \mathrm{~K}$ $\left(100 \mathrm{~km} \mathrm{~h}^{-1}\right.$ for all except tilting and diabatic terms, which are contoured every $100 \mathrm{~K}(100 \mathrm{~km} \mathrm{~h})^{-1}$. Line segment $C D$ in $\mathrm{d}$ ) represents the location of future cross sections.

theory with reflectivities appearing at and west of the eastern edge of the frontal boundary, near the $240-\mathrm{km}$ distance, where isentropic surfaces $\leq 300 \mathrm{~K}$ slope steeply to the surface (Fig. 4.7b).

The quantification of the baroclinicity along the mid-Atlantic coast, as well as the force that generated vertical circulations, required use of the Miller (1948) frontogenesis equation (3.5). The temperature gradient off the coast of South Carolina was consistent with an area of frontogenesis at 1200 UTC 03 October 2015 that was being advected both 

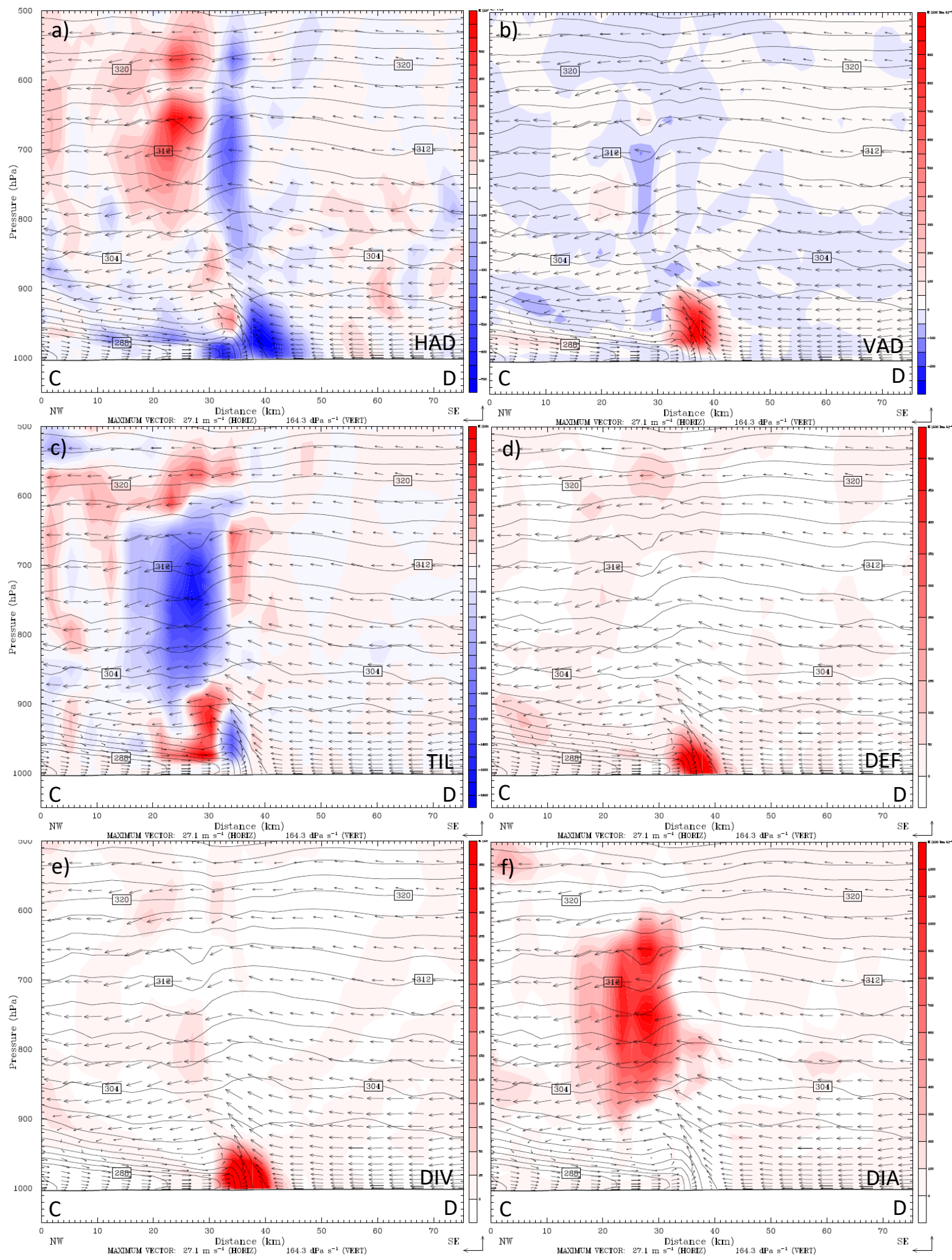

Figure 4.9. Cross sections CD (Fig. 4.8d) showing a) horizontal advective, b) vertical advective, c) tilting, d) deformation, e) divergence, and f) diabatic frontogenesis $\left[\mathrm{K}\left(100 \mathrm{~km} \mathrm{~h}^{-1}\right.\right.$; shaded; positive red, negative blue], potential temperature ( $\mathrm{K}$; contoured), and circulation vectors $\left[\mathrm{m} \mathrm{s}^{-1}, \mathrm{dPa} \mathrm{s}^{-1}\left(1 \mathrm{dPa} \mathrm{s}^{-1}=10^{-3} \mathrm{mb} \mathrm{s}^{-1}\right)\right.$ ] at 1200 UTC 03 October 2015 from WRF domain 2. 
horizontally (Fig. 4.8a) and vertically (Fig. 4.8b). All terms from the Miller (1948) frontogenesis equation (3.5) contributed to frontogenesis along the coast (Fig. 4.8c-f). The horizontal advection of the horizontal potential temperature gradient was negative near the surface due to deformation (Fig. 4.8d) and divergence (Fig. 4.8e), meaning the isentropes were beginning to spread apart near the surface (Fig. 4.9a). The mid-levels displayed a slightly stronger positive horizontal advection of the potential temperature gradient (Fig. 4.9a), which was likely due to diabatic heating (Fig. 4.8f).

The vertical advection of the horizontal potential temperature gradient was positive from 950-900 hPa (Fig. 4.9b), suggesting a strengthening boundary at that level. The region of vertical advective frontogenesis corresponded to an area of negative contribution from the tilting term (Fig. 4.9c), which was no surprise due to the negative horizontal advection of the gradient in the same location. The boundary seemed to be strengthening overall at the $950-900 \mathrm{hPa}$ level because of the positive tilting term (Fig. 4.8c) just west of the strongest potential temperature gradient at the surface (Fig. 4.9c). Thus, the positive contribution from the tilting term outweighed the negative contribution close to the surface to maintain low-level frontogenesis (Fig. $4.8 \mathrm{c}, 4.9 \mathrm{c}$ ).

Frontogenesis was maximized in the low levels as a result of strong deformation (Fig. 4.8d, 4.9d) and divergence (Fig. 4.8e, 4.9e). Deformation and divergence contributed to the thermally direct circulation below $900 \mathrm{hPa}$ (Fig. 4.9e), helping to organize the band of convection, and likely causing the area of negative tilting at $950 \mathrm{hPa}$ (Fig. 4.9c). Precipitation was likely enhanced within and on the cold side of the front due to the intensification of the frontal circulation (Bosart et al. 1972) caused by the increased deformation and divergence. The precipitation field (Fig. 4.7a) was consistent with the 


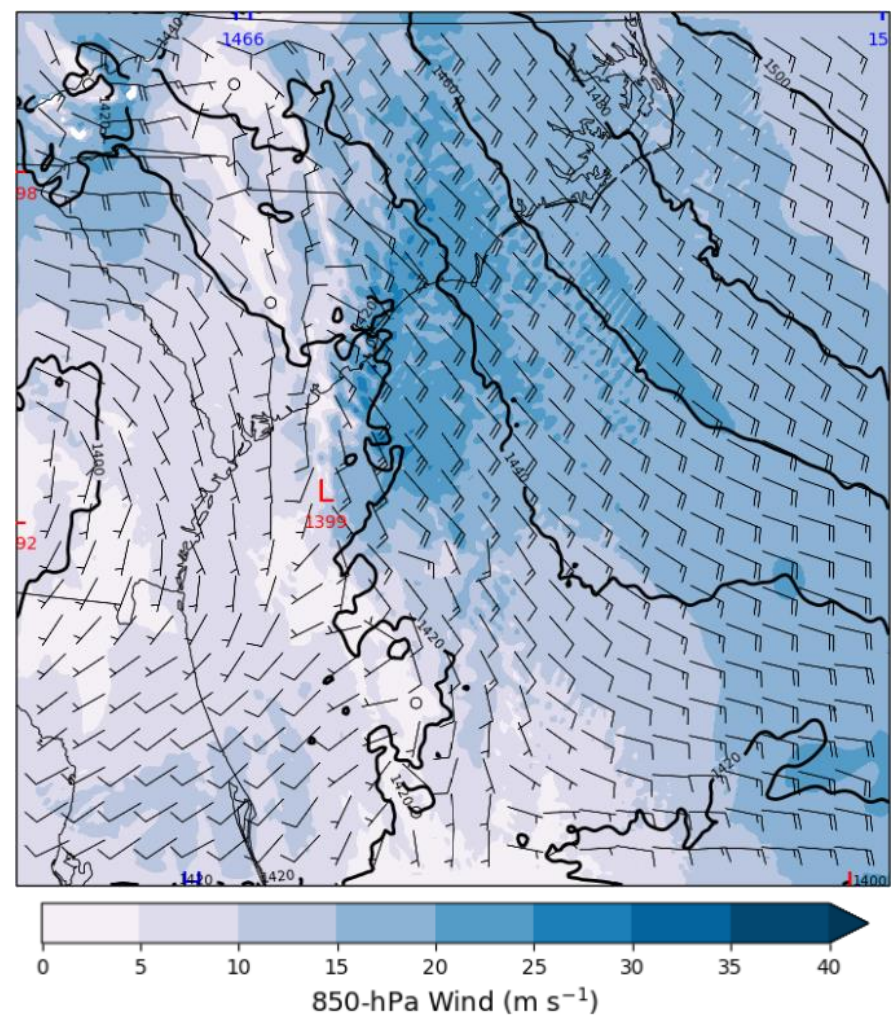

Figure 4.10. WRF results for domain 2 of the control simulation at 1200 UTC 03 October 2015 displaying 850-hPa height (gpm; contoured) and wind $\left(\mathrm{m} \mathrm{s}^{-1}\right)$.

positive diabatic term that dominated mid-level frontogenesis (Fig. 4.8f) due to condensational warming. In response to the mid-level diabatic heating, isentropes slope down between 850 and $650 \mathrm{hPa}$ at about the 27-km mark in the red region of frontogenesis (Fig. 4.9f). The differential diabatic heating was a result of the heavy rainfall and enhanced the mid-level temperature gradient (Atallah and Bosart 2003; Colle 2003) southeast to northwest from 900 to $600 \mathrm{hPa}$ (Fig. 4.9f).

A few bands of precipitation at 1200 UTC 03 October 2015 appear just east of the low pressure off the coast of Georgia, stretching north, along and nearly parallel to the low-level baroclinic zone (Fig. 4.7a), consistent with an area of 850-hPa winds 


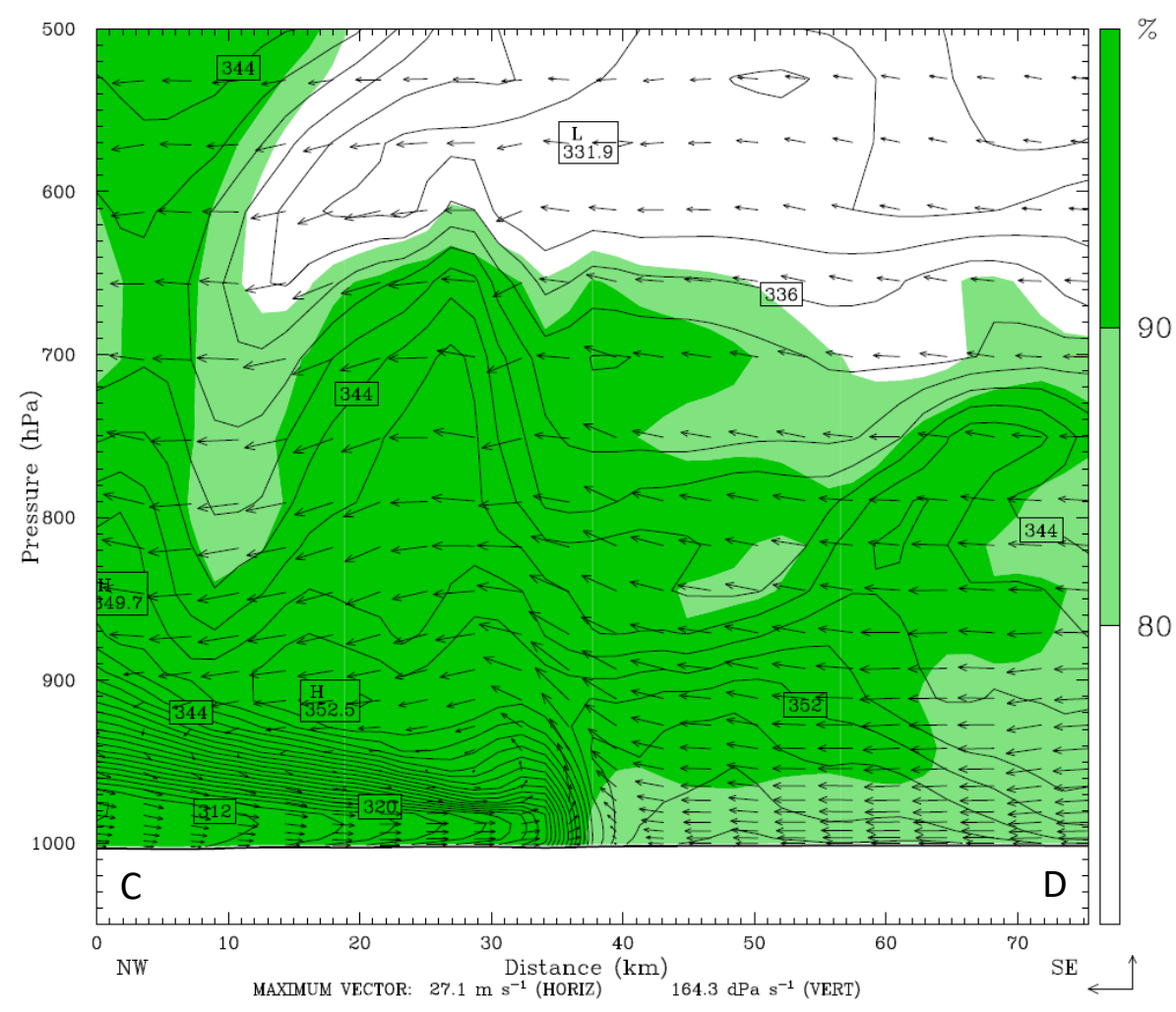

Figure 4.11. Cross section CD (Fig. 4.8d) at 1200 UTC 03 October 2015 showing equivalent potential temperature ( $\mathrm{K}$; contoured), relative humidity (shaded), and circulation vectors $\left[\mathrm{m} \mathrm{s}^{-1}, \mathrm{dPa} \mathrm{s}^{-1}\left(1 \mathrm{dPa} \mathrm{s}^{-1}=\right.\right.$ $\left.10^{-3} \mathrm{mb} \mathrm{s}^{-1}\right)$ ] from WRF domain 2 .

greater than $20 \mathrm{~m} \mathrm{~s}^{-1}$ (Fig. 4.10). Potential instability (PI) is found where equivalent potential temperature $\left(\theta_{e}\right)$ decreases with height. In regions of PI, upright convection can result from a layer being lifted, obtaining a statically unstable lapse rate. Further, environments with PI can aid elevated convection with the warm conveyer belt of a cyclone interacting with cold, dry air aloft to produce negative $\theta_{e}$ lapse rates (Halcomb and Market 2003). An area of potential instability (PI) was located near the location of maximum rainfall, $\mathrm{C}$, from 850 to $550 \mathrm{hPa}$ (Fig. 4.11). The same location was nearly saturated through $500 \mathrm{hPa}$, with RH values above 90\% (Fig. 4.11). The pattern was 


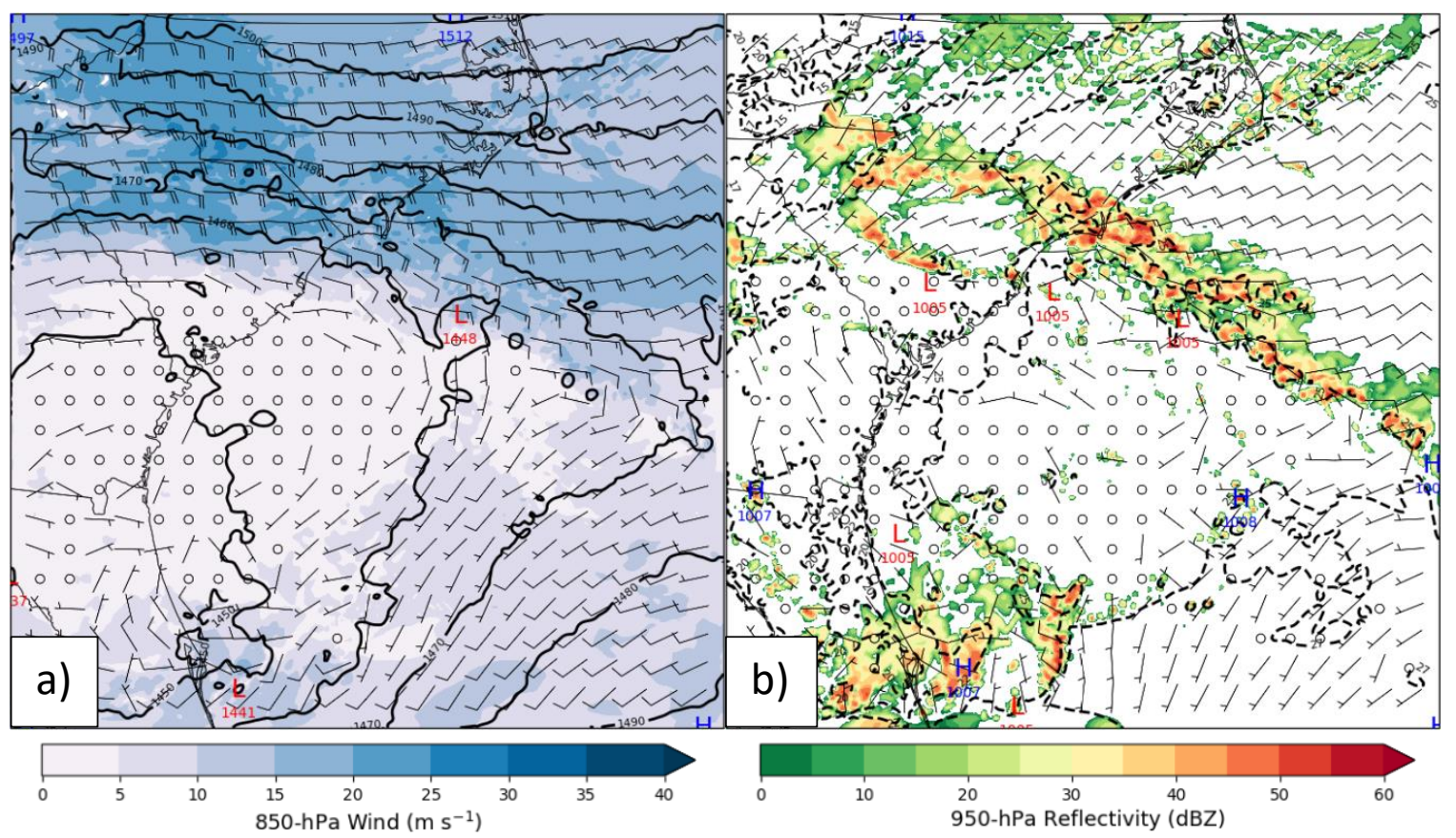

Figure 4.12. WRF results for domain 2 of the control simulation at 0000 UTC 05 October 2015 displaying a) 850-hPa height (gpm; contoured), 850-hPa wind $\left(\mathrm{m} \mathrm{s}^{-1}\right)$, and b) 2-m temperature ( ${ }^{\circ} \mathrm{C}$; dashed), 950-hPa reflectivity ( $\mathrm{dBZ}$; shaded), and $10-\mathrm{m}$ wind $\left(\mathrm{m} \mathrm{s}^{-1}\right)$.

supportive of convective overturning in the mid-levels. Additionally, convection near point $\mathrm{C}$ was elevated due to the stable layer near the surface (Fig. 4.11).

An aid to the lift and convective initiation at the location of the coastal front, winds at $850 \mathrm{hPa}$ were southeasterly approaching $30 \mathrm{~m} \mathrm{~s}^{-1}$ as they crossed nearly perpendicular to the boundary at 1200 UTC 03 October 2015 (Fig. 4.12a). As the event progressed, the low-level jet that was associated with the deep low pressure over the southeastern United States began to rotate similar to the propagation of the upper-level jet (Fig. 4.3a, b). The low-level jet went from a north-south orientation (Fig. 4.10) to an eastwest orientation by 0000 UTC 05 October 2015 (Fig. 4.12a). The precipitation field remained aligned with the low-level jet throughout the event. By 0000 UTC 05 October 

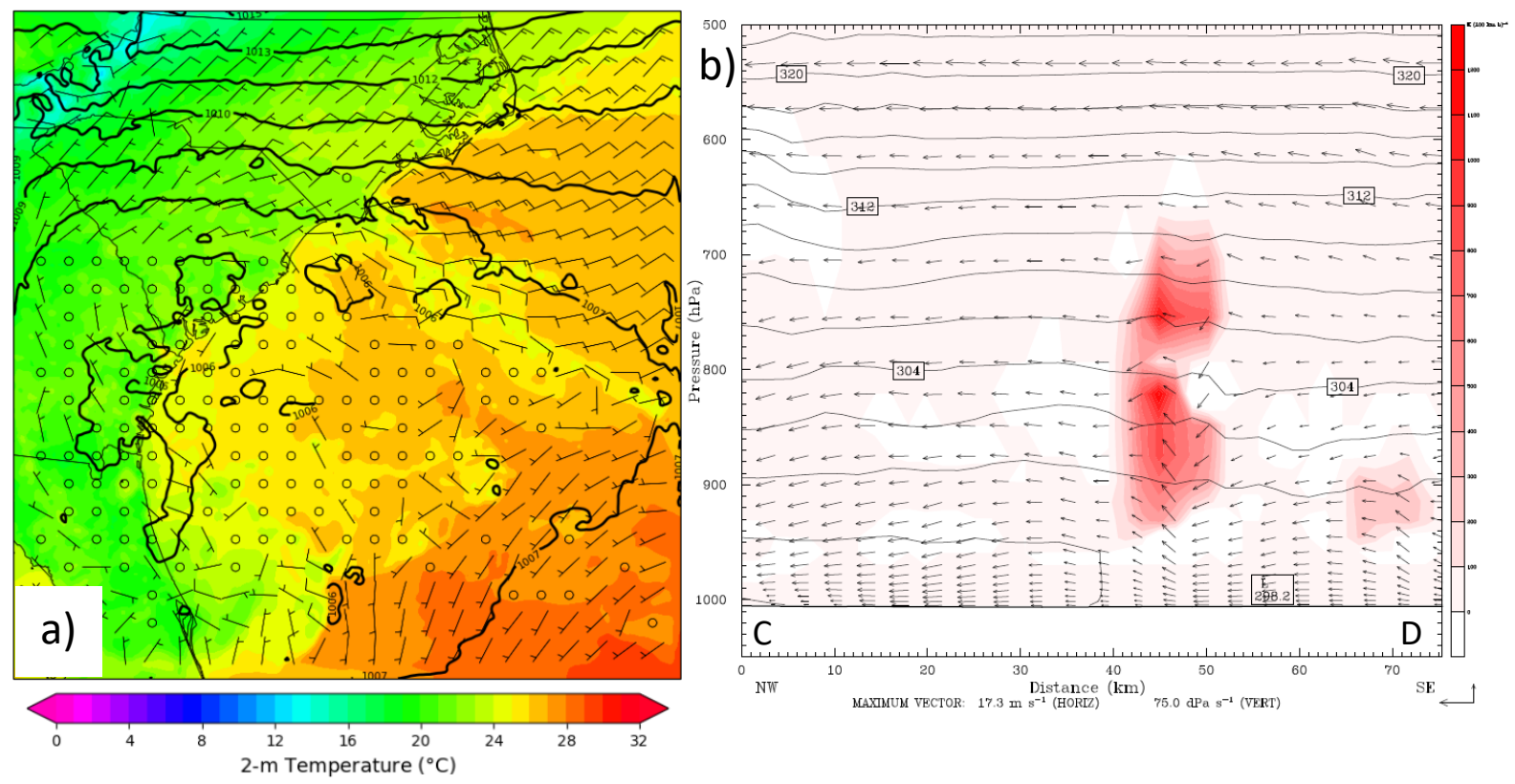

Figure 4.13. WRF results for domain 2 of the control simulation at 0000 UTC 05 October 2015 displaying a) sea level pressure ( $\mathrm{hPa}$; contoured), 2-m temperature $\left({ }^{\circ} \mathrm{C}\right.$; shaded), and $10-\mathrm{m}$ wind $\left(\mathrm{m} \mathrm{s}^{-1}\right)$, and $\left.\mathrm{b}\right)$ cross section CD (Fig. 4.8d) representing diabatic frontogenesis [ $\mathrm{K}\left(100 \mathrm{~km} \mathrm{~h}^{-1}\right.$; shaded, positive red], potential temperature ( $\mathrm{K}$; contoured), and circulation vectors $\left[\mathrm{m} \mathrm{s}^{-1}, \mathrm{dPa} \mathrm{s}^{-1}\left(1 \mathrm{dPa} \mathrm{s}^{-1}=10^{-3} \mathrm{mb} \mathrm{s}^{-1}\right)\right]$.

2015, the area of rainfall and its intensity began to diminish as the extratropical cyclone and Hurricane Joaquin moved northeast (Fig. 4.12b).

The coastal front remained stationary near South Carolina and North Carolina throughout the event, as the extratropical cyclone maintained northerly flow over the mid-Atlantic states east of the Appalachians with southeasterly flow just off the coast. The WRF simulation showed the front at its strongest from 1200 UTC 03 October 2015 (Fig. 4.6). As the event unfolded, the boundary weakened and became difficult to identify using the WRF simulation due to the cool surface temperatures (Fig. 4.13a) associated with the area of precipitation (Fig. $4.12 \mathrm{~b}$ ). A temperature change of $5^{\circ} \mathrm{C}$ over a $100 \mathrm{~km}$ 
range was still visible along the coast of North Carolina and just south of the convection at 0000 UTC 05 October 2015 (Fig. 4.13a).

A cross section just south of the precipitation field, consistent with the area of the previous cross sections, displayed only one isentrope sloping down to the surface with no evidence of a frontal circulation (Fig. 4.13b). The weak boundary still provided lift in the region, while diabatic heating contributed to frontogenesis in the mid-levels (Fig. 4.13b). However, the tilting term of the Miller (1948) frontogenesis equation (3.5) contributed to frontolysis in the mid-levels at 0000 UTC 05 October 2015 (not shown), which counteracted the frontogenesis at that time off the coast of South Carolina. Additionally, there was no evidence of divergence or deformation in the low levels to contribute to frontogenesis (not shown). With a diminishing frontal boundary, the intense precipitation event came to an end for the South Carolina region by 0000 UTC 05 October 2015 in the WRF simulation, consistent with the precipitation accumulation for Florence, South Carolina (Fig. 4.1a).

\subsubsection{Trajectories}

To further support the hypothesis that the coastal front initiated the convection that led to copious amounts of precipitation in South Carolina, a trajectory analysis was completed. Parcel trajectories began at a location of heavy rainfall (upwards of $900 \mathrm{~mm}$ for the event) near the South Carolina coast (Fig. 4.1b). Trajectories worked backwards 6 hours from 1200 UTC 03 October 2015, when the coastal front was at its strongest and the precipitation rate was beginning to increase (Fig. 4.1a). Five trajectories were 


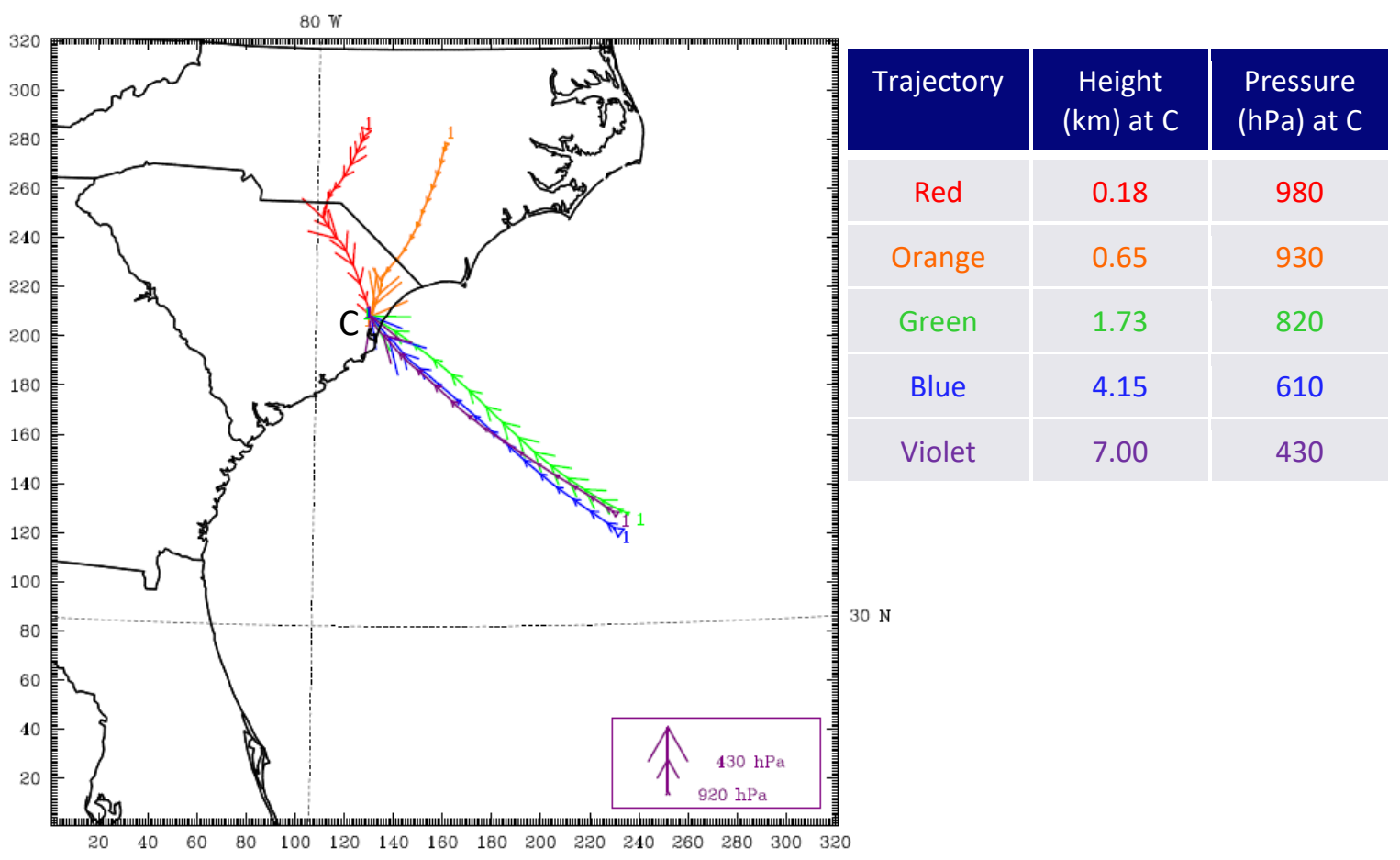

Figure 4.14. Parcel trajectories for the control simulation of the 2015 South Carolina flood event beginning at 1200 UTC 03 October 2015 in the region of the heaviest rainfall, point C, and working backward six hours. The trajectories are colored by height above ground/pressure of the parcel at point $\mathrm{C}$. The arrows on the trajectory represent the height of the parcel at that time, with larger arrows representing a higher level.

initialized at different levels in the atmosphere above the location of heavy rain (Fig.

4.14), corresponding to different eta levels in the WRF simulation. Closest to the surface, parcels were approaching South Carolina from the north, while parcels at $820 \mathrm{hPa}$ and above were approaching from the southeast (Fig. 4.14). This pattern was consistent with the atmospheric conditions on the synoptic scale and mesoscale: an upper-level low over the southeastern United States and a frontal boundary along the coast. The parcels near to the surface were representative of cool, drier air to the north. Concerned with the rainfall in the area, it was necessary to further analyze the parcels that represented warm, moist 
air. Thus, the parcel closest to the surface that originated southeast of South Carolina (green trajectory in Fig. 4.14) was investigated.

As the parcel approached the South Carolina coast, it began to increase in height (as seen by the increase in arrow size in Fig. 4.15a). The location of the coastal front (Fig. 4.15b) corresponded to the parcel's change in altitude (Fig. 4.15a) and circulation vectors indicated lift just southeast and along the frontal boundary from the surface through 850 $\mathrm{hPa}$ (Fig. 4.15b). There was no question of parcel lift over the frontal boundary, but further investigation was required to determine if the parcel was being lifted enough to initiate convection.

The parcel's environment was viewed through soundings at three locations: southeast of the frontal boundary, along the front, and northeast of the frontal boundary (Fig. 4.15a). The parcel traveled from the southeast location to the northeast location in a 10-minute period, so the soundings represent the change in the parcel's environment every 5 minutes beginning at 1130 UTC 03 October 2015. At the southeast location, the parcel had a pressure of $938 \mathrm{hPa}$, temperature of $23^{\circ} \mathrm{C}$, and was in a conditionally stable layer (Fig. 4.15c). The atmospheric column had a precipitable water value of $54 \mathrm{~mm}$, winds were near $35 \mathrm{knots}$ from the southeast at $938 \mathrm{hPa}$, and the parcel was gradually lifting at $25 \mathrm{~cm} \mathrm{~s}^{-1}$. At this point, the parcel had not reached its level of free convection (LFC), as that was calculated to be much higher at $605 \mathrm{hPa}$ (Fig. 4.15c).

As the parcel reached the coastal front, its pressure and temperature decreased to $908 \mathrm{hPa}$ and $21^{\circ} \mathrm{C}$, respectively (Fig. $4.15 \mathrm{~d}$ ). Along the frontal boundary, the atmospheric column had a precipitable water value of $56 \mathrm{~mm}$ and a saturated level was present between 950 and $700 \mathrm{hPa}$. An inversion was evident below $925 \mathrm{hPa}$, with veering winds 

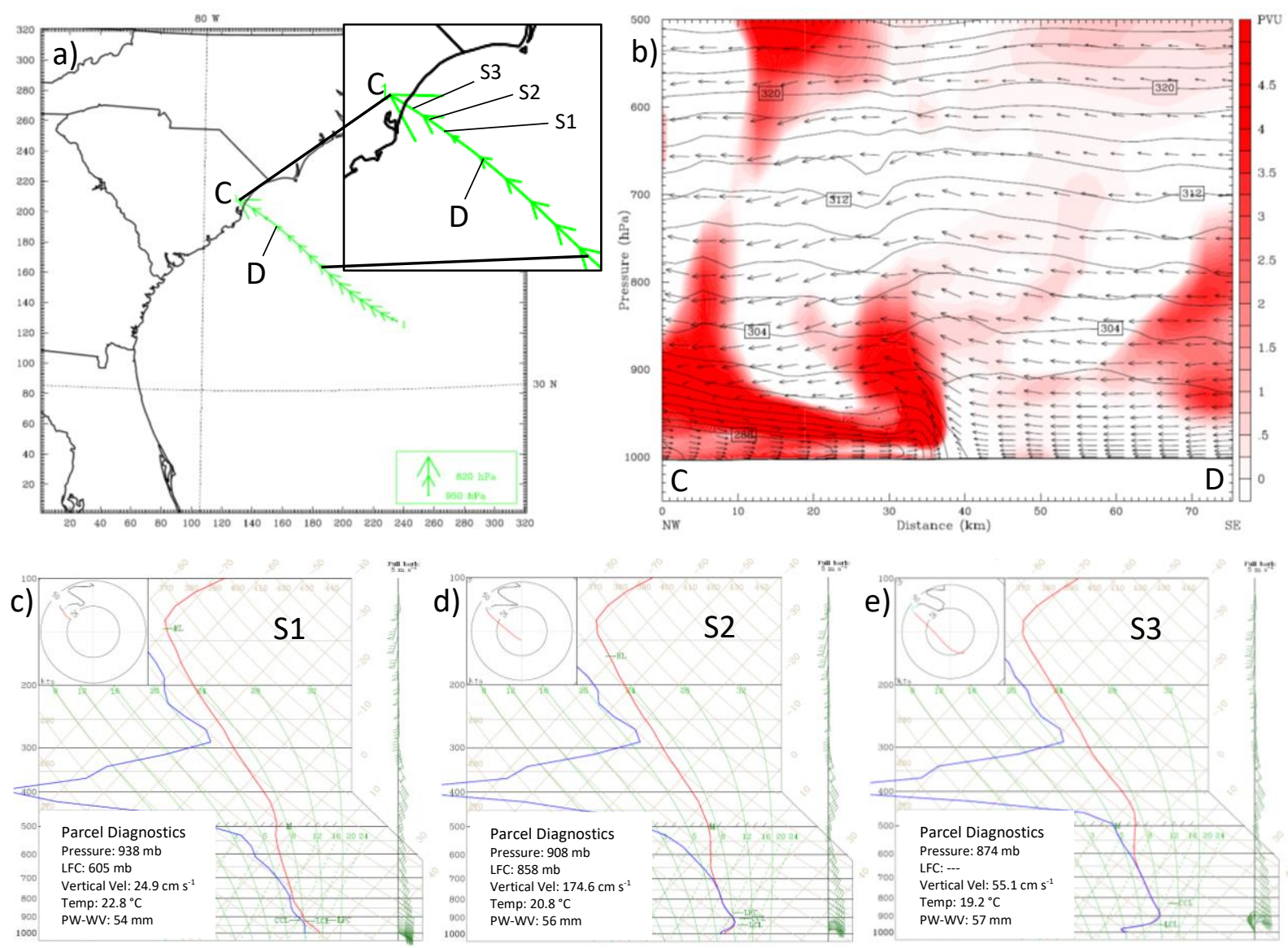

Figure 4.15. The control simulation of the 2015 South Carolina flood event is represented by a) a parcel trajectory beginning at 1200 UTC 03 October 2015 in the region of the heaviest rainfall, point C, at $820 \mathrm{mb}$ and working backward six hours. The arrows on the trajectory represent the height of the parcel at that time, with larger arrows representing a higher level. Points $C$ and D (same as Fig. 4.8d) on the trajectory represent the region of the b) cross section at 1200 UTC 03 October 2015, which includes potential vorticity (PVU; red shaded), potential temperature (K; contoured), and circulation vectors ( $\mathrm{m} \mathrm{s}^{-1}, \mathrm{dPa} \mathrm{s}^{-1}$ ). Soundings for the parcel location along the trajectory were created in 5-minute intervals beginning with $\mathrm{c}$ ) S1 at 1130 UTC, d) S2 at 1135 UTC, and e) S3 at 1140 UTC 03 October 2015 to represent the environment of the parcel before, during, and after crossing the frontal boundary, evident in b). Diagnostics for the parcel of interest are included with each sounding to represent the state of the parcel at that time.

(Fig. 4.15d). The parcel was above the inversion at the time, with strong southeasterly winds and a vertical velocity of $175 \mathrm{~cm} \mathrm{~s}^{-1}$. The parcel was much closer to its LFC, which was only $858 \mathrm{hPa}$ (Fig. 4.15d), and was in a moist absolutely unstable layer (MAUL; Bryan and Fritsch 2000). 
By 1140 UTC 03 October 2015, the parcel had reached the northwest side of the frontal boundary and had a pressure of $874 \mathrm{hPa}$ (Fig. 4.15e), placing it within the thermally direct circulation associated with the front (Fig. 4.15b). The inversion from 975 to $900 \mathrm{hPa}$ was stronger than the inversion within the front; $6-7^{\circ} \mathrm{C}$ difference across 75 $\mathrm{hPa}$ (Fig. 4.15e) compared to only $2^{\circ} \mathrm{C}$ across $50 \mathrm{hPa}$ (Fig. 4.15d), with surface temperatures about $5^{\circ} \mathrm{C}$ cooler northeast of the boundary. The column water vapor was similar with $57 \mathrm{~mm}$ of precipitable water (Fig. 4.15e). However, the environment was saturated from $650 \mathrm{hPa}$ to the surface (Fig. 4.15e). Winds were veering with height in the inversion layer, but the parcel of interest was located above the inversion in an area of strong, southeasterly winds advecting it into South Carolina. The parcel continued to cool as it passed by the front and was down to $19^{\circ} \mathrm{C}$ at 1140 UTC 03 October 2015 (Fig. 4.15e). The parcel's vertical velocity had weakened, but was still $55 \mathrm{~cm} \mathrm{~s}^{-1}$, and its LFC could not be calculated because it had surpassed that level.

As the parcel approached and passed by the coastal front, it lifted, cooled, became encompassed in a saturated environment, and reached its LFC. The parcel environment remained conditionally stable/unstable for the 10-minute period, but the parcel's level was saturated along (Fig. 4.15d) and northeast (Fig. 4.15e) of the front suggesting an unstable environment. The parcel analysis concluded that the coastal front was able to lift parcels to their LFC, initiating the convection that was advected into South Carolina. Further, the convection was elevated in nature because the parcel reached its LFC above a stable surface layer (Fig. 4.15e). 


\subsubsection{Role of the Tropical Cyclone}

The first step in determining the impact of Hurricane Joaquin (2015) on the South Carolina flood event was to compare precipitation from the WRF control simulation to that of the WRF simulation with the tropical cyclone vortex removed from initial conditions. For the 108-hour WRF control simulation of the South Carolina flood, over $900 \mathrm{~mm}$ of rain fell over parts of South Carolina with a maximum of $907.04 \mathrm{~mm}$ just southeast of Florence, SC (Fig. 4.1b). When the vortex of Hurricane Joaquin was removed from the WRF simulation, an area off the coast of Wilmington, North Carolina received intense rainfall from 1200 UTC 03 October to 0600 UTC 04 October 2015 (Fig. 4.16a) with a maximum of $965.27 \mathrm{~mm}$ of rain for the entire 108-hour period (Fig. 4.16b). South Carolina received very little rain after the removal of Joaquin because the axis of the heaviest precipitation shifted to be more north-south (Fig. 4.16b) rather than the eastwest pattern of the control simulation (Fig. 4.1b).

The east displacement and north-south orientation of the precipitation field was caused by the change in position of the upper-level low and the change in the deformation zone over the western Atlantic. Without the tropical cyclone, the upper low was slightly north (Fig. 4.17a) of its location in the control simulation (Fig. 4.3a) at 1200 UTC 03 October 2015. As a result, the upper-level jet was positioned over South Carolina (Fig. 4.17a) instead of Georgia (Fig. 4.3a) at 1200 UTC 03 October 2015. The displacement of the right entrance region of the jet and its associated upper-level divergence (Fig. 4.13a) was the underlying cause of the eastward shift in precipitation. The north-south orientation of the precipitation was the result of the axis of dilatation 

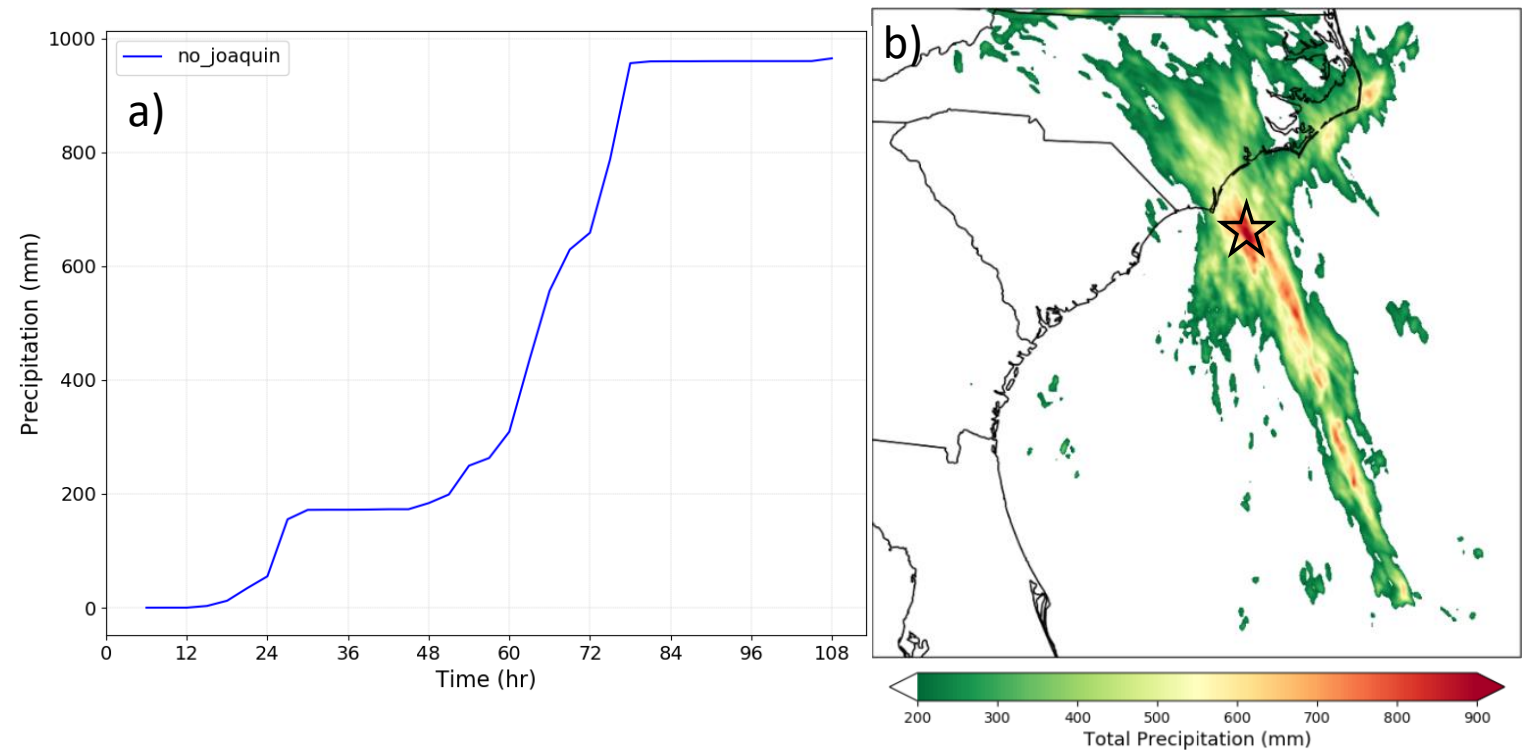

Figure 4.16. Precipitation ( $\mathrm{mm}$ ) for the South Carolina flood event from 0000 UTC 01 October 2015 to 1200 UTC 05 October 2015 for domain 2 of the WRF simulation with the vortex of Hurricane Joaquin removed a) at the location of maximum precipitation, plotted as accumulation with time and b) as the event total for the region. The transparent star represents the location of maximum precipitation.

associated with the deformation zone between the upper-level low and the region of low pressure near Bermuda (Fig. 4.17a).

As the event progressed, the upper-level low displayed the same behavior as the control simulation with a westward tilt in the associated jet streaks (Fig. 4.17b). Stalled by the negative PV advection by the irrotational wind, caused by the latent heat release in the region of deep convection, the upper-level low remained over Georgia until 05 October 2015 when it was able to progress east. Thus, the tropical cyclone was not the main reason for the stagnant synoptic pattern. However, the intense convection period was roughly 6 hours shorter in duration without Hurricane Joaquin (Fig. 4.16a). This 


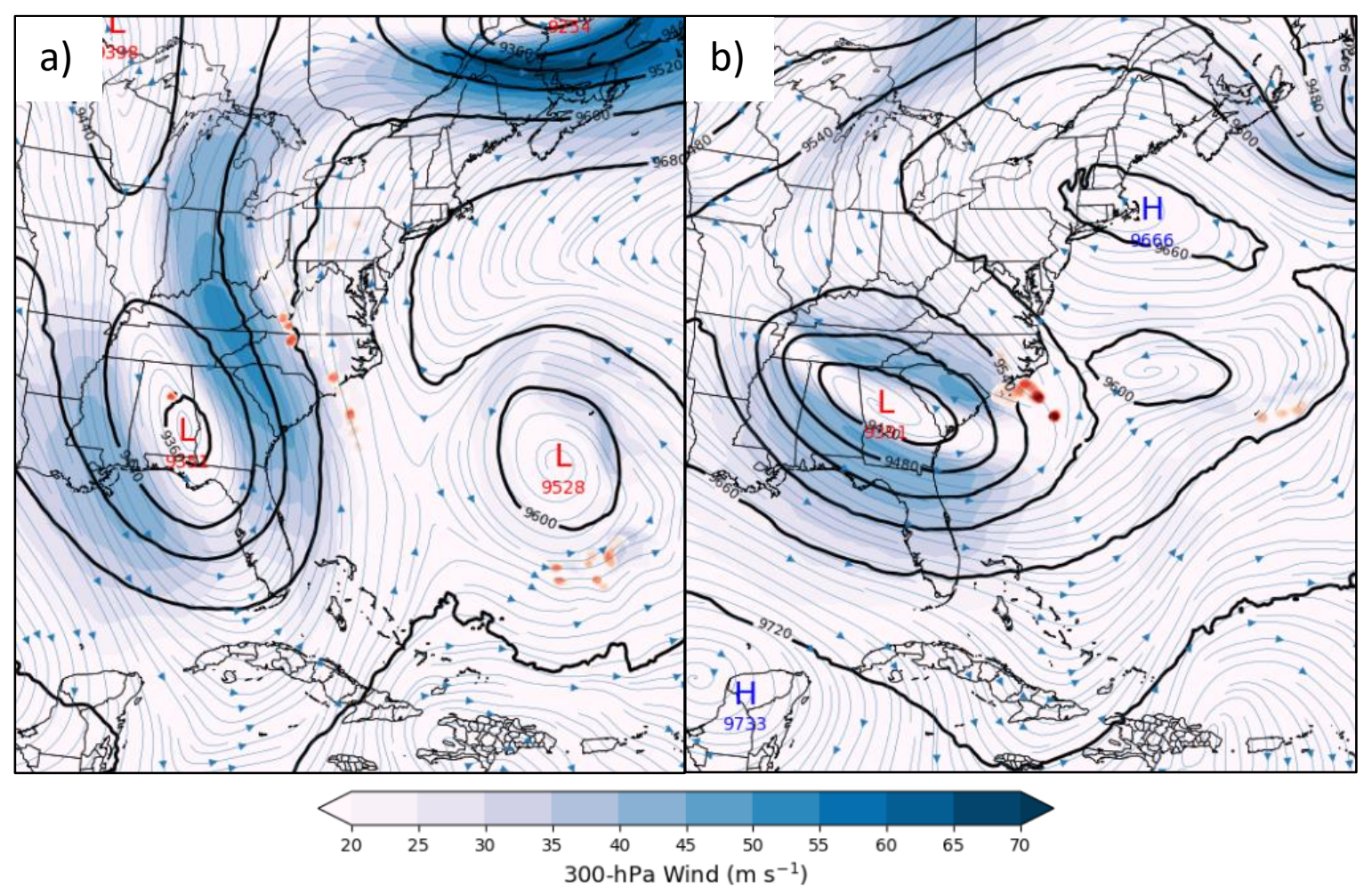

Figure 4.17. WRF domain 1 300-hPa height (gpm; thick contoured), wind ( $\mathrm{m} \mathrm{s}^{-1}$; shaded; thin streamlines), and divergence $\left(10^{-5} \mathrm{~s}^{-1}\right.$; contoured; positive red) at a) 1200 UTC 03 October 2015 and b) 1200 UTC 04 October 2015 of the simulation with the removal of the tropical cyclone vortex.

suggested a minor impact from the outflow and deformation zone on the propagation of the extratropical cyclone, similar to the conclusions of Marciano and Lackmann (2017).

Moisture availability could have also led to a shorter period of convection for the event without Hurricane Joaquin's presence. Precipitable water values were comparable between the control simulation and the simulation with no tropical cyclone vortex at 1200 UTC 03 October 2015, except that the moisture plume with values near $70 \mathrm{~mm}$ was roughly 3 times the meridional span when Joaquin was removed (Fig. 4.18a). The elongated plume from the eastern Bahamas to North Carolina showed more similarities to 


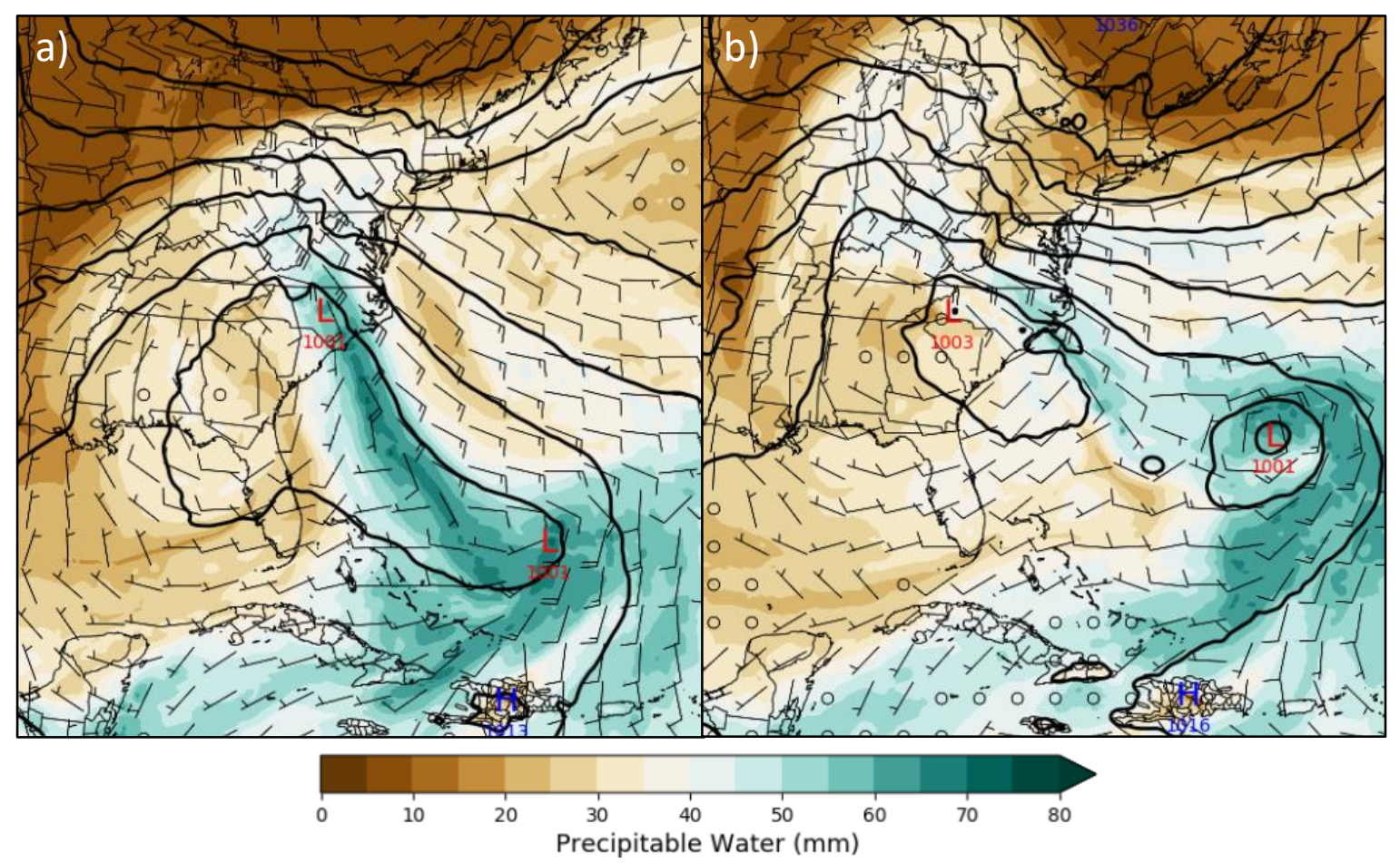

Figure 4.18. WRF results for domain 1 of the simulation with the tropical cyclone vortex removed at a) 1200 UTC 03 October 2015 and b) 1200 UTC 04 October 2015 displaying precipitable water (mm; shaded), sea level pressure (hPa; contoured), and 850-hPa wind $\left(\mathrm{m} \mathrm{s}^{-1}\right)$.

an atmospheric river than the plume from the control simulation (Fig. 4.4a) and corresponded with the longer, narrower swath of precipitation (Fig. 4.16b). The increased moisture was likely the reason for higher precipitation totals (Fig. 4.16b), but by 1200 UTC 04 October 2015 the precipitable water amounts were nearly $20 \%$ lower (Fig. 4.18b) than those of the control simulation (Fig. 4.4b).

Similar conclusions were drawn from IVT, with a stronger moisture transport jet (near $800 \mathrm{~kg} \mathrm{~m}^{-1} \mathrm{~s}^{-1}$, stretching further south) evident at 1200 UTC 03 October 2015 near the Carolinas without Joaquin (Fig. 4.19a) than that of the control simulation (Fig. 4.5a). Similar IVT values were displayed for both simulations at 1200 UTC 04 October 2015 


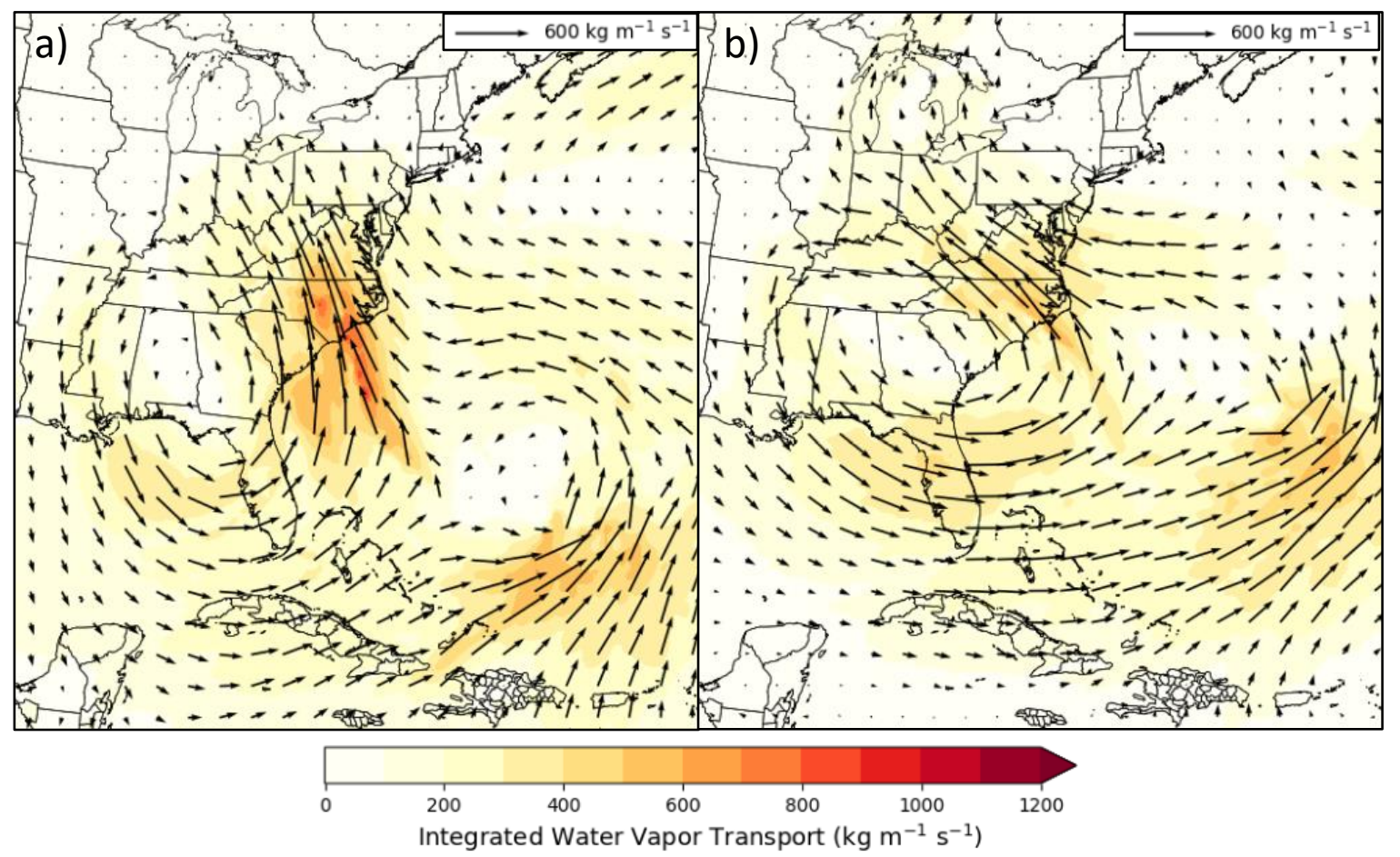

Figure 4.19. WRF results for domain 1 of the simulation with the removal of the tropical cyclone vortex at a) 1200 UTC 03 October 2015 and b) 1200 UTC 04 October 2015 displaying integrated water vapor transport (IVT; $\mathrm{kg} \mathrm{m}^{-1} \mathrm{~s}^{-1}$ ).

(Fig. 4.5b, Fig. 4.19b), except the transport jet was already north of the region of maximum precipitation in the simulation with the cyclone vortex removed (Fig. 4.19b). The consistent outflow from Hurricane Joaquin may have supplied enough moisture and slowed the progression of the extratropical cyclone just enough to prolong the event by 6 hours, but the tropical cyclone may have also limited the total amount of moisture available to the South Carolina region.

The removal of Hurricane Joaquin also impacted the coastal front. A quick comparison of the baroclinic zone along the same line as the intense rainfall in the control 

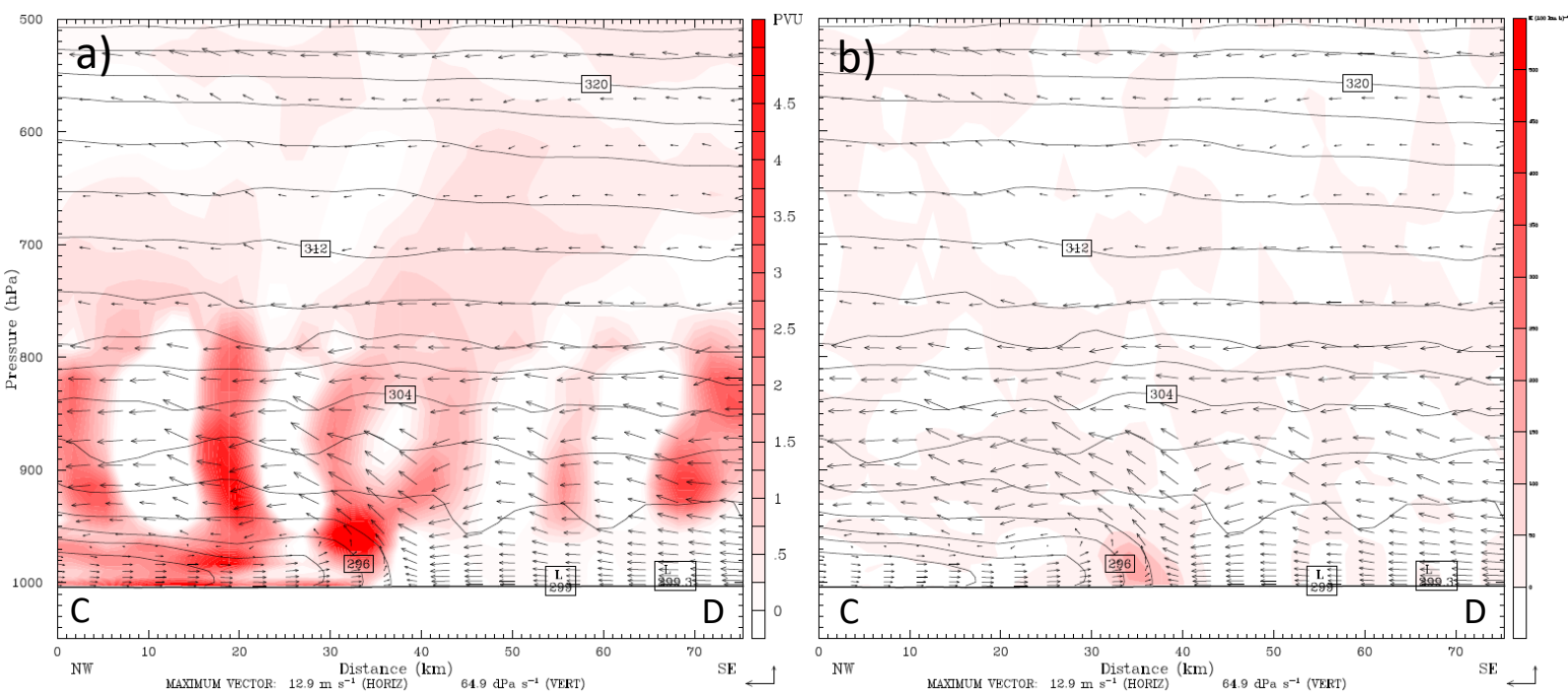

Figure 4.20. WRF cross sections for domain 2 of the simulation with the tropical cyclone vortex removed at 1200 UTC 02 October 2015 for line segment CD (Fig. 4.8d) displaying potential temperature (K; contoured) and circulation vectors $\left[\mathrm{m} \mathrm{s}^{-1}, \mathrm{dPa} \mathrm{s}^{-1}\left(1 \mathrm{dPa} \mathrm{s}^{-1}=10^{-3} \mathrm{mb} \mathrm{s}^{-1}\right)\right]$ with a) potential vorticity (PVU; shaded red) and b) deformation frontogenesis [ $\mathrm{K}\left(100 \mathrm{~km} \mathrm{~h}^{-1}\right.$; shaded, positive red].

simulation showed the presence of a boundary with a thermally direct circulation after the tropical cyclone vortex was removed (Fig. 4.20a). However, the boundary in that location was its strongest at 1200 UTC 02 October 2015 (Fig. 4.20a) and was not visible after 0000 UTC 03 October 2015. Even at its strongest, the coastal front appeared weaker than the boundary in the control simulation (Fig. 4.15b) with greater distances between the steeply sloped isentropes (Fig. 4.20a). Deformation (Fig. 4.20b) and divergence led to frontogenesis near the surface, but values were weaker than those found in the control simulation (Fig. 4.9d). The front was visible with 2-m temperature and 10-m wind, along with an area of cold air damming in the Carolinas at 1200 UTC 02 October 2015 (Fig. 4.21a). 


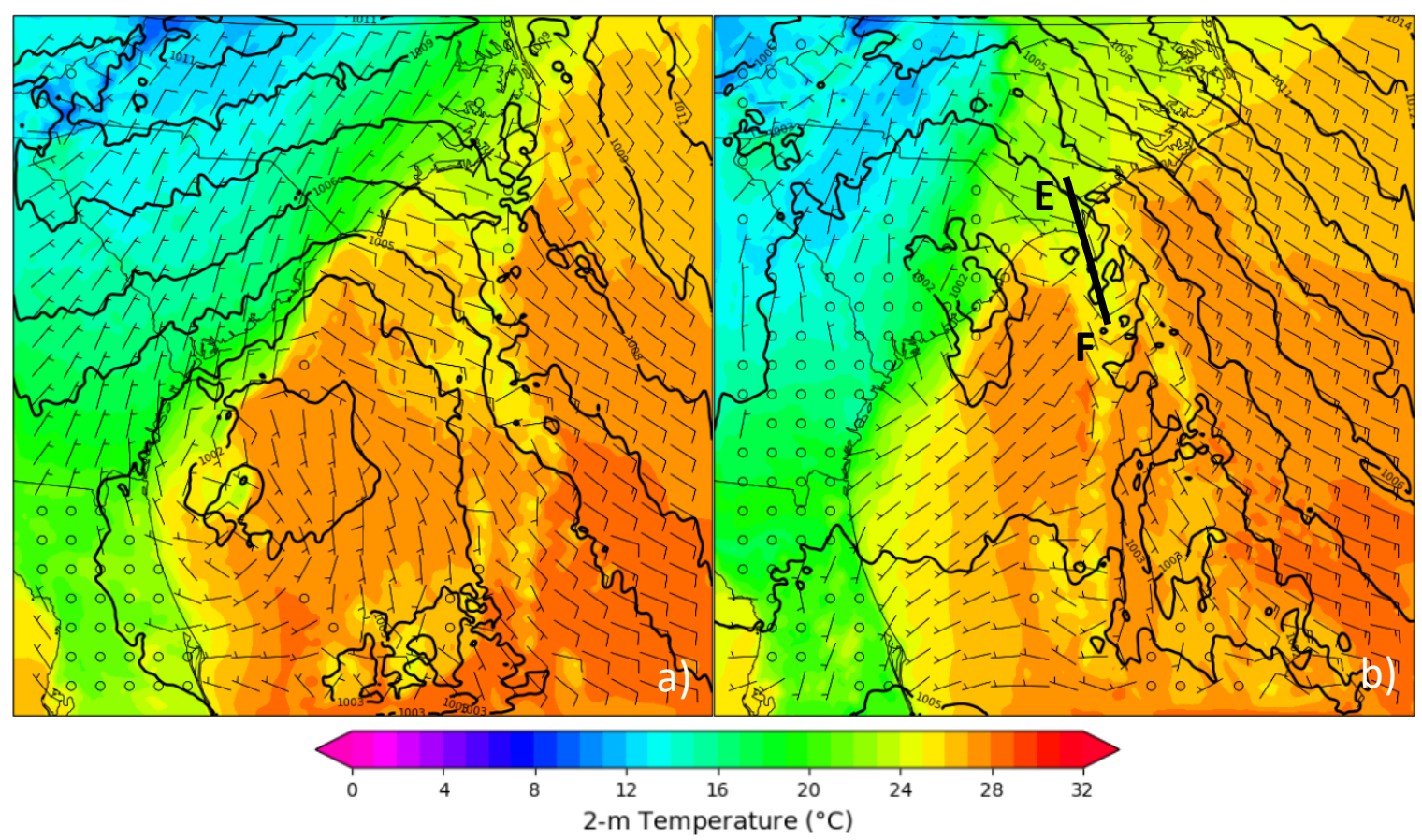

Figure 4.21. WRF results for domain 2 of the simulation with the tropical cyclone vortex removed at a) 1200 UTC 02 October 2015 and b) 1200 UTC 03 October 2015 displaying sea level pressure (hPa; contoured), 2-m temperature ( $\left({ }^{\circ} \mathrm{C}\right.$; shaded), $10-\mathrm{m}$ wind $\left(\mathrm{m} \mathrm{s}^{-1}\right)$, and line segment $\mathrm{EF}$.

When analyzing the mesoscale environment at the time of convective initiation resulting in over $600 \mathrm{~mm}$ of rain for the simulation without Joaquin (Fig. 4.16a), it was clear that the coastal front was weaker at 1200 UTC 03 October 2015 (Fig. 4.21b) than it was when Joaquin was present (Fig. 4.6). Surface temperatures over the Atlantic Ocean, southeast of the boundary, were $2^{\circ} \mathrm{C}$ warmer with the presence of Joaquin. The biggest difference between simulations was the magnitude of the cold air damming; nearly a $6^{\circ} \mathrm{C}$ difference was found for the region east of the Appalachian Mountains between the control simulation (Fig. 4.6) and the simulation with the tropical cyclone vortex removed (Fig. 4.21b). Cold air damming was likely less intense for the simulation without Hurricane Joaquin because the surface low was positioned further north over the South 


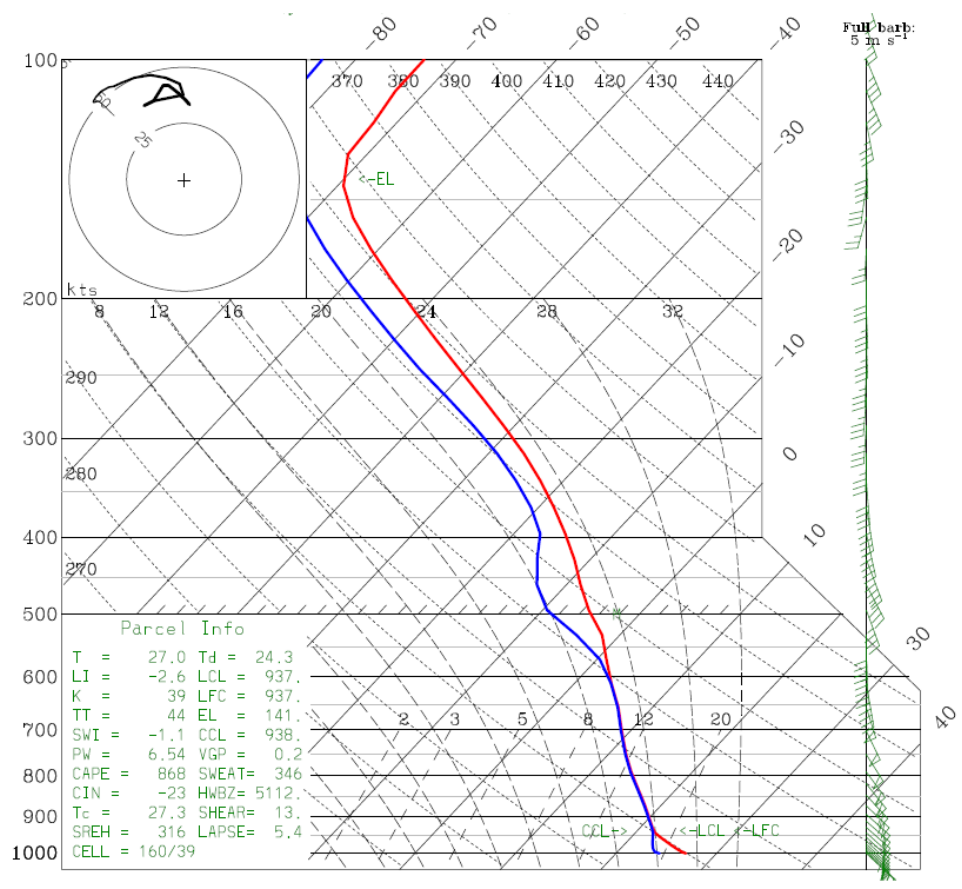

Figure 4.22. WRF sounding from domain 2 of the simulation with the tropical cyclone vortex removed at 1200 UTC 03 October 2015 for the location of maximum precipitation showing temperature $\left({ }^{\circ} \mathrm{C}\right.$; red), dew point $\left({ }^{\circ} \mathrm{C}\right.$; blue), and wind $\left(\mathrm{m} \mathrm{s}^{-1}\right)$.

Carolina coast (Fig. 4.21b), rather than off the coast of Georgia (Fig. 4.6). The position of the surface low over South Carolina allowed for easterly flow to dominate the eastern half of North Carolina, limiting the region of northerly flow east of the Appalachians (Fig. 4.21b). Less intense cold air damming and slightly cooler surface temperatures off the mid-Atlantic coast resulted in a weaker temperature gradient.

Southeasterly flow persisted across the axis of precipitation (Fig. 4.21b), which suggested no frontal circulation and no elevated convection. A sounding at the location of maximum precipitation (Fig. 4.16b) at the time of convective initiation showed a saturated layer from 950 to $600 \mathrm{hPa}$ with strong southeasterly winds from the surface to the mid-levels (Fig. 4.22). The low-level environment was conditionally unstable to the 
surface with a low LFC of $937 \mathrm{hPa}$. Additionally, there was no inversion present near the surface, which suggested surface-based convection. The intensity of the precipitation was aided by the high precipitable water $(65 \mathrm{~mm})$ in the column (Fig. 4.22). The environmental characteristics remained the same through 0600 UTC 04 October 2015 (not shown), the entirety of the intense precipitation period.

\subsubsection{Terrain Influence}

A deeper analysis of the formation of the coastal front and how it impacted the precipitation event in South Carolina required the use of experimental WRF simulations to manipulate the height of the Appalachian Mountains. The location and orientation of the Appalachians makes the East Coast prone to cold air damming that often results in a low-level baroclinic zone along the coast (Bosart 1975; Richwien 1980; Stauffer and Warner 1987). Cold air damming was present during the South Carolina flood event in 2015 (Fig. 4.6). Thus, removing the Appalachian Mountains could result in warmer air in the location of cold air damming, causing a weaker temperature gradient. A weaker coastal front may not have provided the lift needed to initiate convection off the coast of South Carolina, resulting in little to no precipitation. Alternatively, increasing the height of the Appalachians could strengthen the cold air damming and resultant coastal front, leading to increased lift and more intense convection for South Carolina.

The extreme differences in total accumulated precipitation from the experimental simulation with terrain removed (Fig. 4.23a) and that with terrain height doubled (Fig. 4.23b) supported the hypotheses. Analyzing the location of maximum precipitation in 


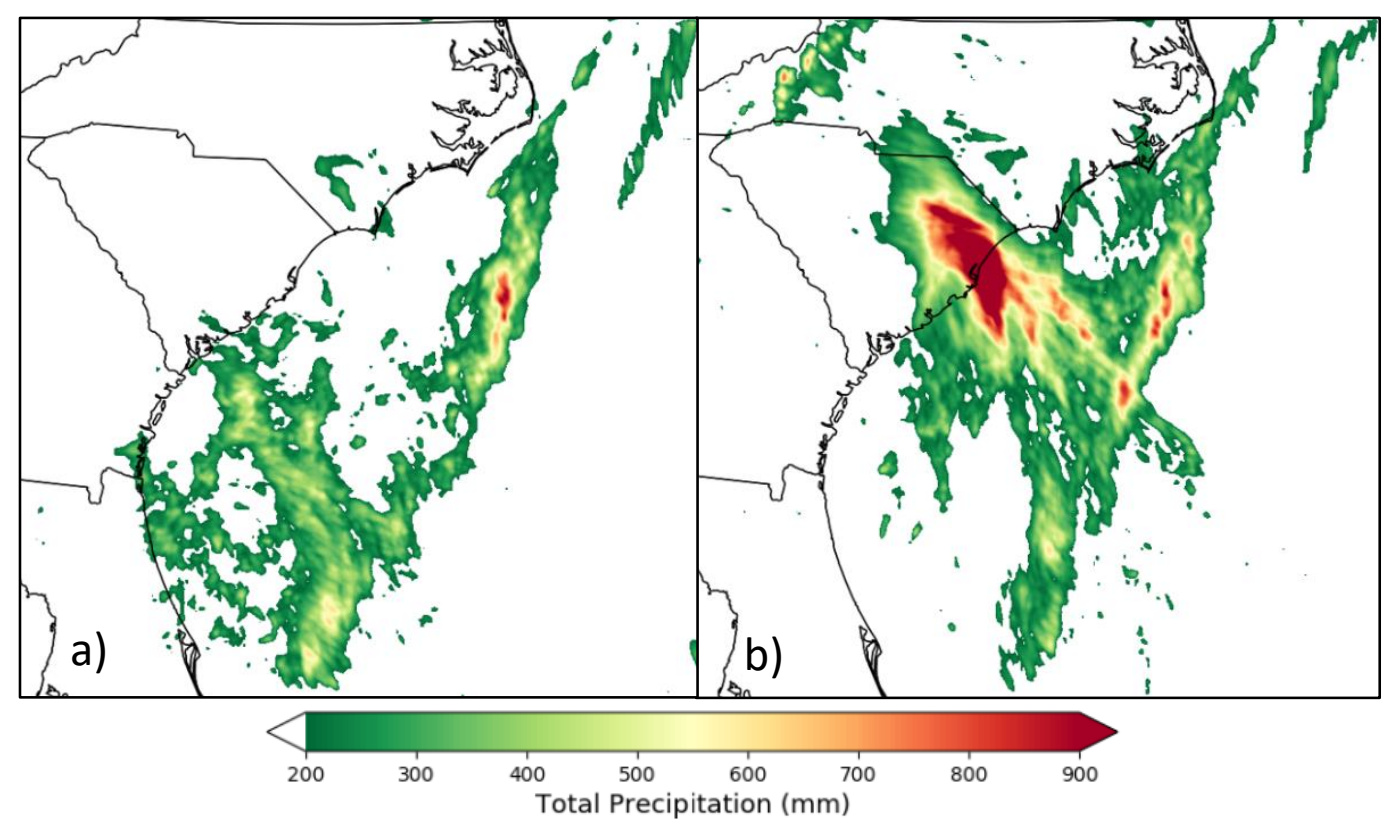

Figure 4.23. Total accumulated precipitation $(\mathrm{mm})$ from WRF domain 2 for the simulation with a) terrain removed and b) terrain doubled.

South Carolina found in the WRF control simulation (Fig. 4.1b), little to no precipitation fell when terrain was removed (0-200 mm; Fig. 4.23a). The same region in South Carolina, from Florence to areas southeast off the coast, received $1460 \mathrm{~mm}$ (57 inches) when the Appalachians were doubled in height (Fig. 4.23b). That equates to 1.6 times the maximum precipitation found in the control simulation (Fig. 4.1b) when the mountains were stretched to $4,000 \mathrm{~m}$.

On the synoptic scale, removing terrain caused the upper-level low and associated jet to shift south over the Gulf of Mexico (Fig. 4.24a). As a result, the right entrance region of the jet and the resultant upper-level divergence was placed over northeast Florida with a lack of upper-level support for precipitation over South Carolina (Fig. 4.24a). Doubling terrain resulted in an upper-level environment (Fig. 4.24b) like that of 


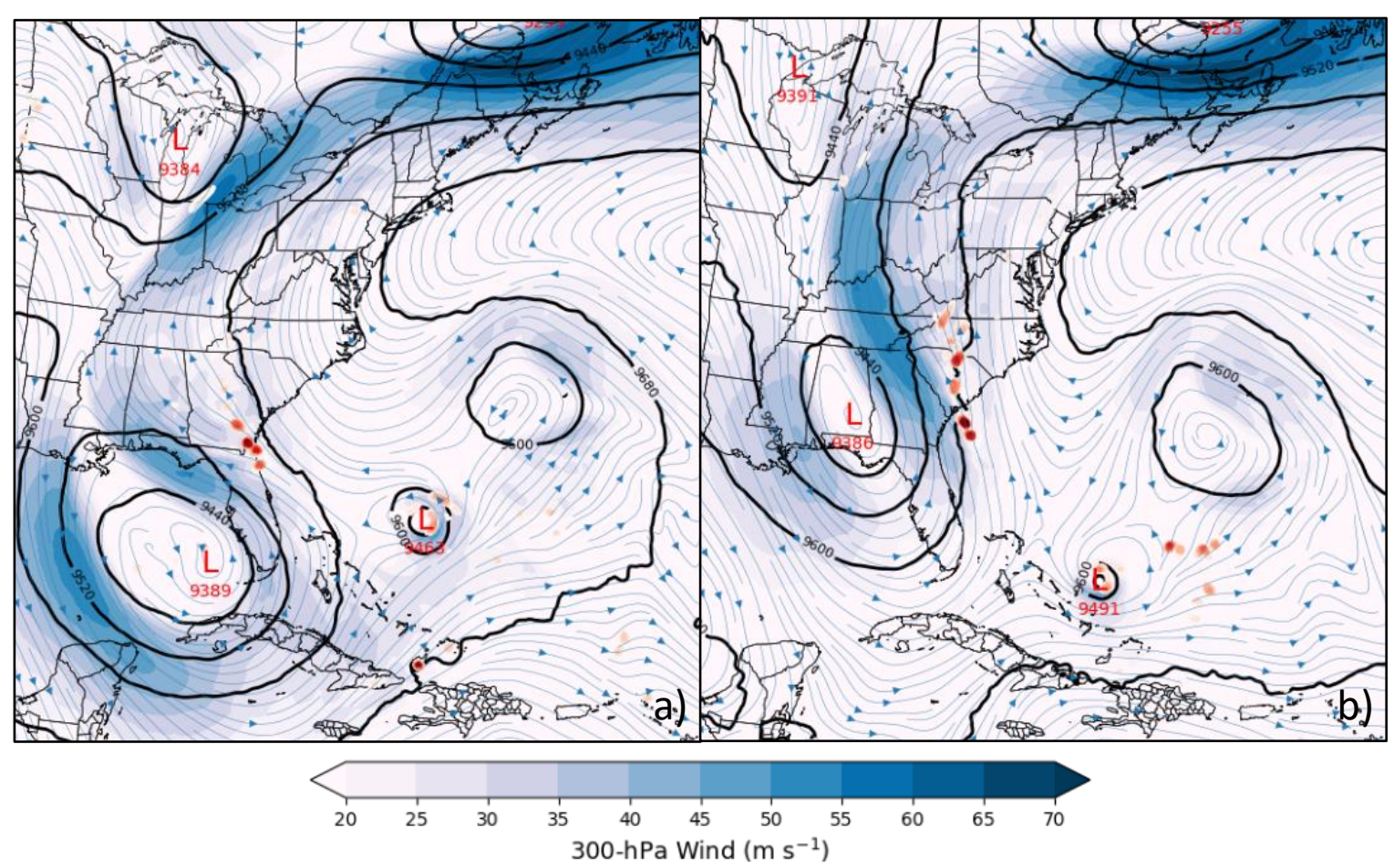

Figure 4.24. WRF domain 1300 -hPa height (gpm; thick contoured), wind ( $\mathrm{m} \mathrm{s}^{-1}$; shaded; thin streamlines), and divergence $\left(10^{-5} \mathrm{~s}^{-1}\right.$; contoured; positive red) at 1200 UTC 03 October 2015 for the simulation with a) terrain removed and $b$ ) terrain doubled.

the WRF control simulation for the event (Fig. 4.3a). A moisture plume developed along the axis of dilatation between the extratropical and tropical cyclones in both terrainmanipulated simulations. When terrain was removed, the moisture plume was directed into Georgia with precipitable water values approaching $70 \mathrm{~mm}$ (Fig. 4.25a) due to warmer sea-surface temperatures (not shown). The plume resembled that found in the control simulation (Fig. 4.4a) when terrain was doubled (Fig. 4.25b).

As a result of the synoptic scale differences between the simulations, Hurricane Joaquin was able to track closer to the United States when terrain was removed, with a similar track between the control and the simulation with terrain doubled (Fig. 4.26a). 


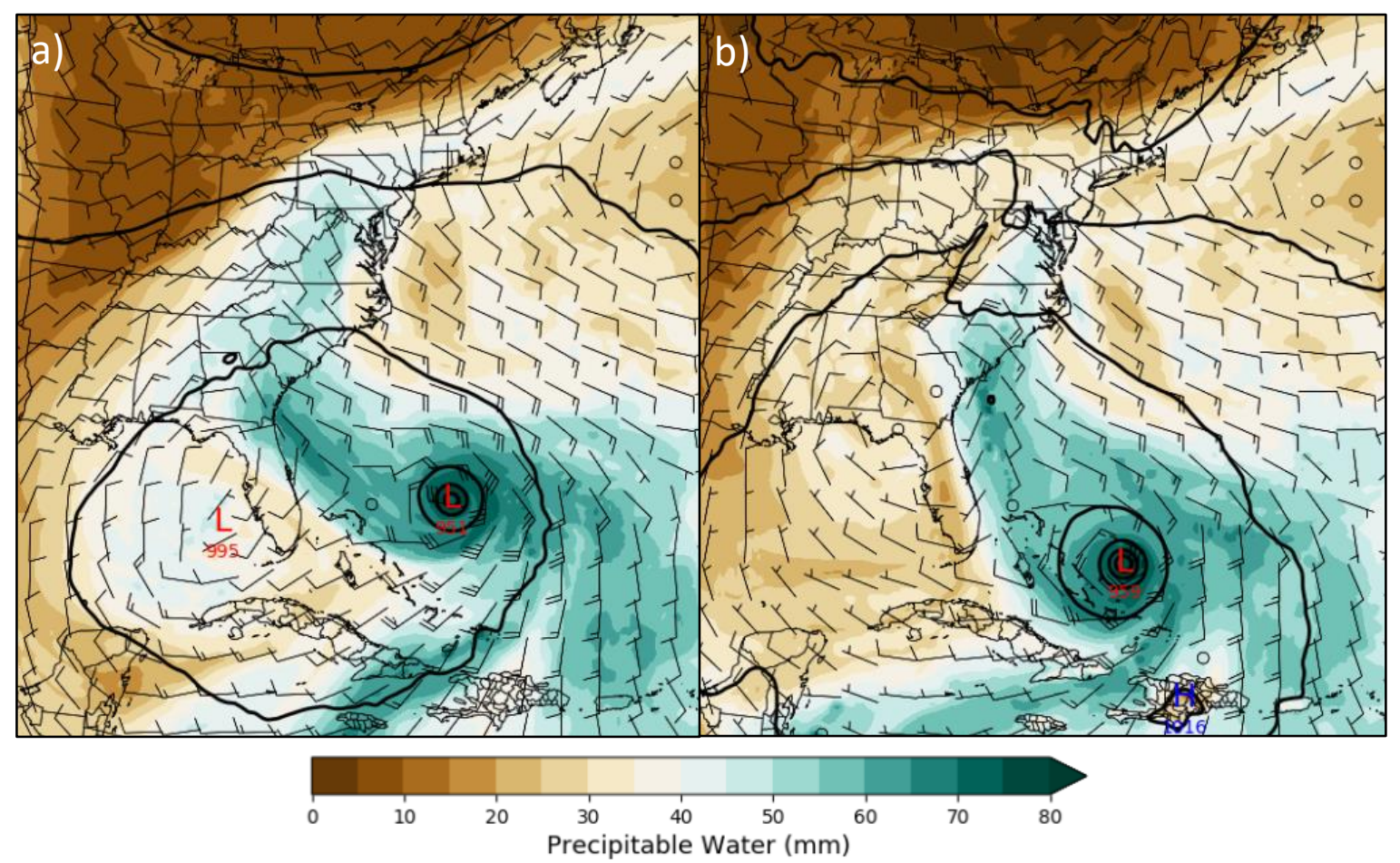

Figure 4.25. WRF results for domain 1 at 1200 UTC 03 October 2015 displaying precipitable water (mm; shaded), sea level pressure ( $\mathrm{hPa}$; contoured), and 850-hPa wind ( $\mathrm{m} \mathrm{s}^{-1}$ ) for the simulation with a) terrain removed and $b$ ) terrain doubled.

The upper-level ridge over the northwestern Atlantic Ocean, an upper-level low near Bermuda, and the extratropical cyclone's position further south over the Gulf of Mexico (Fig. 4.24a) provided a steering flow for the tropical cyclone that guided its position closer to the East Coast when terrain was eliminated (Fig. 4.26b). As the extratropical cyclone propagated east over Florida, both cyclones entered a rotation pattern resembling the Fujiwhara effect (Fujiwhara 1931) before the tropical cyclone began to follow the southeasterly flow of the upper-level ridge (Fig. 4.26b). The WRF simulation ended at 1200 UTC 05 October 2015 with the tropical cyclone located just off the coast of the 

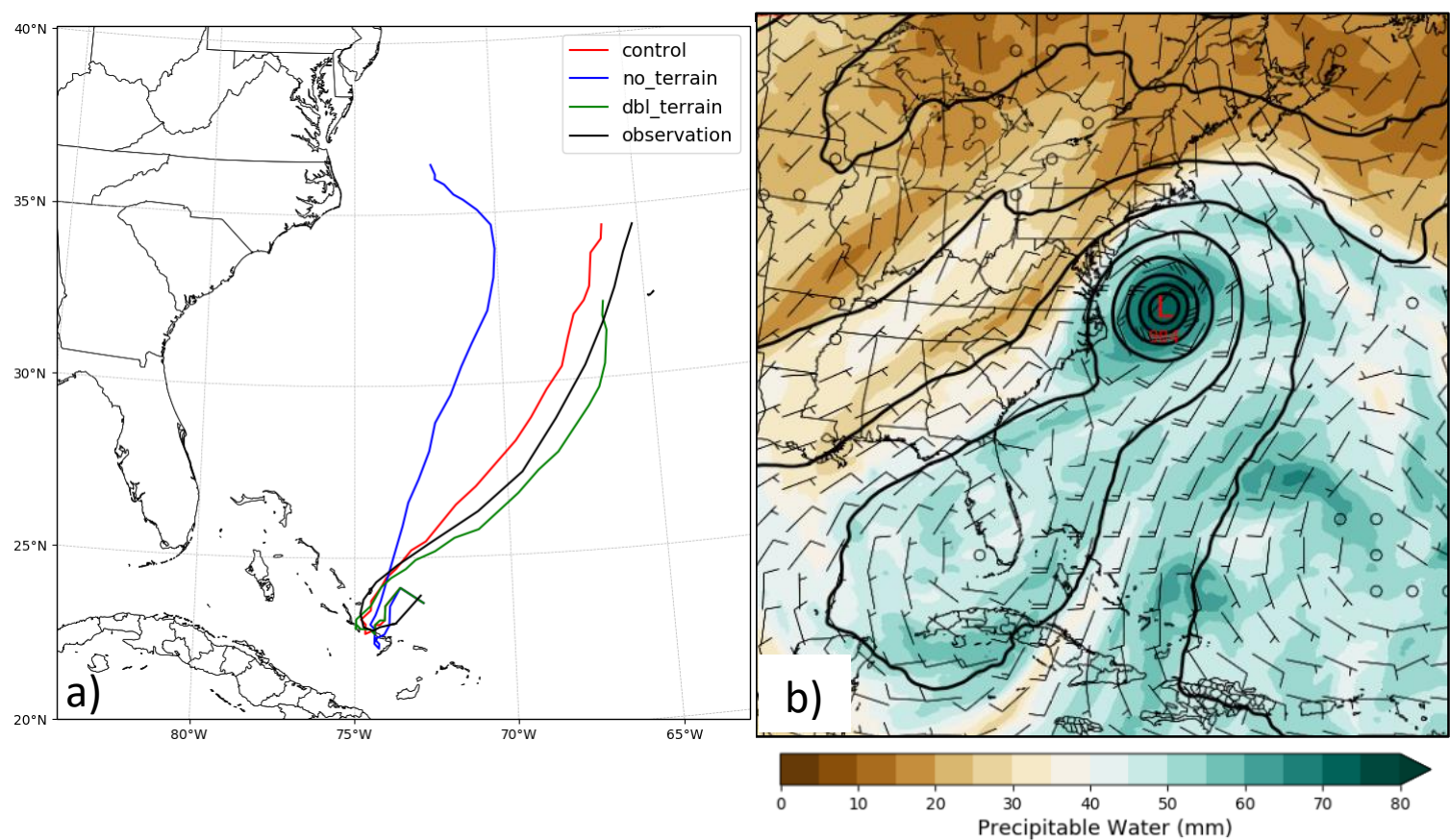

Figure 4.26. WRF results for domain 1 displaying a) the track of Hurricane Joaquin from 0000 UTC 01 October 2015 to 1200 UTC 05 October 2015 and b) precipitable water (mm; shaded), sea level pressure (hPa; contoured), and 850-hPa wind $\left(\mathrm{m} \mathrm{s}^{-1}\right)$ at 1200 UTC 05 October 2015 for the simulation with terrain removed.

mid-Atlantic states (Fig. 4.26b), roughly 6-7 $7^{\circ}$ west of the tropical cyclone position in the other WRF simulations at that time (Fig. 4.26a).

The existence of the Appalachian Mountains had an impact on the synoptic scale flow pattern near the East Coast of the United States, which greatly aided the location and amount of precipitation during the South Carolina flood. However, the terrain influence on the cold air damming and subsequent coastal front was also a factor in the precipitation event. Without the Appalachians, cold air $\left(<12^{\circ} \mathrm{C}\right)$ was able to reach central Georgia and the coldest air was situated over western North Carolina (Fig. 4.27a) as opposed to central North Carolina in the control simulation (Fig. 4.6). The more western 


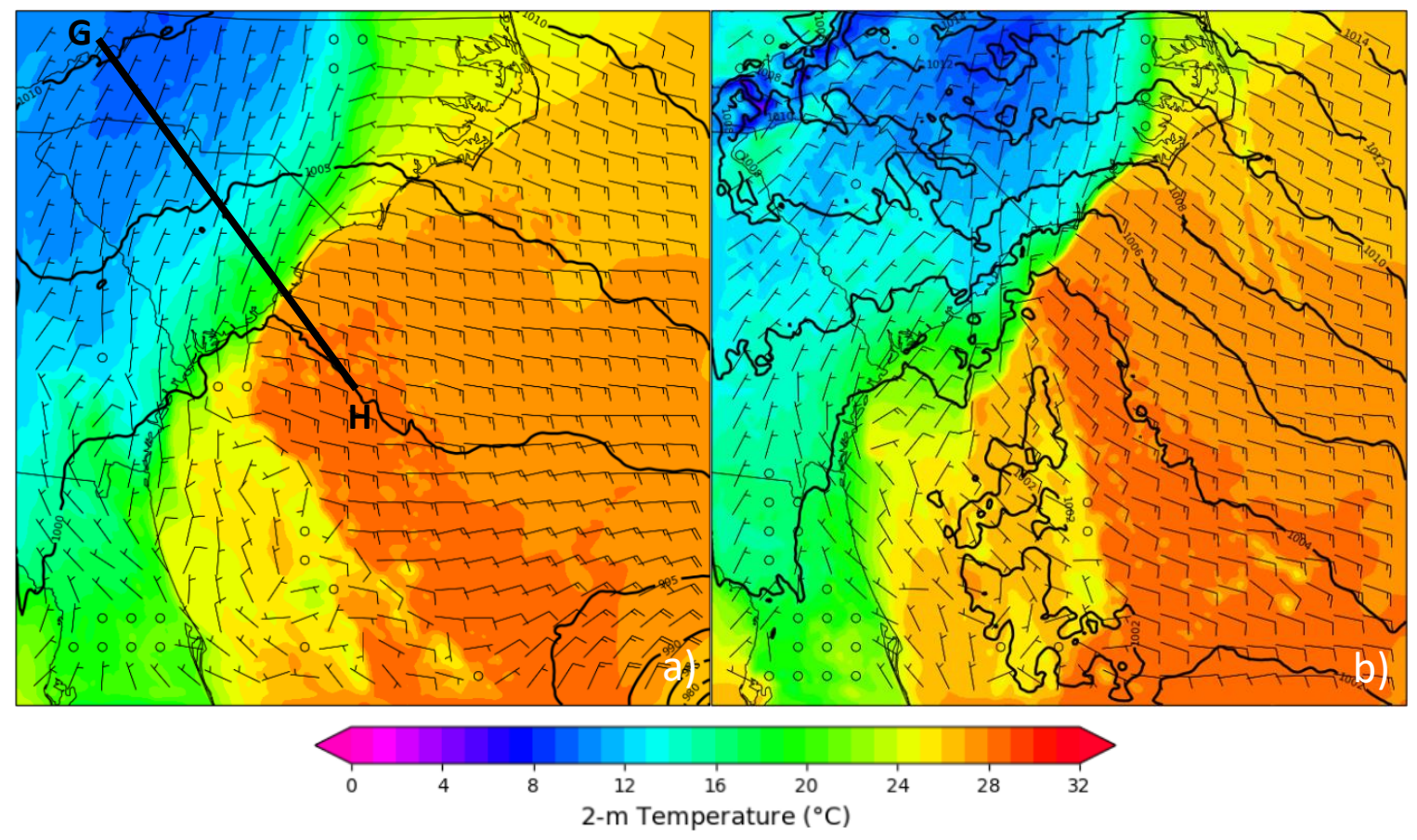

Figure 4.27. WRF results for domain 2 at 1200 UTC 03 October 2015 displaying sea level pressure (hPa; contoured), 2-m temperature $\left({ }^{\circ} \mathrm{C}\right.$; shaded), 10-m wind $\left(\mathrm{m} \mathrm{s}^{-1}\right)$, and line segment $\mathrm{GH}$ for the simulation with a) terrain removed and $b$ ) terrain doubled.

region of cold air allowed for a weaker temperature gradient $\left(\sim 10^{\circ} \mathrm{C} 100 \mathrm{~km}^{-1}\right)$ near the coast (Fig. 4.27a). The cold air damming signature remained consistent between the simulation with terrain height doubled (Fig. 4.27b) and the control simulation (Fig. 4.6). Both terrain-manipulated simulations lack a clear area of low pressure near the surface at 1200 UTC 03 October 2015 (Fig. 4.27a, b), as seen near the coast of Georgia in the control simulation (Fig. 4.6), but southeasterly flow over the western Atlantic and northerly flow over much of the Carolinas remained consistent in all simulations.

Cross sections were able to clearly show the differences in terrain height and coastal front location between the simulations with terrain removed (Fig. 4.28a) and 

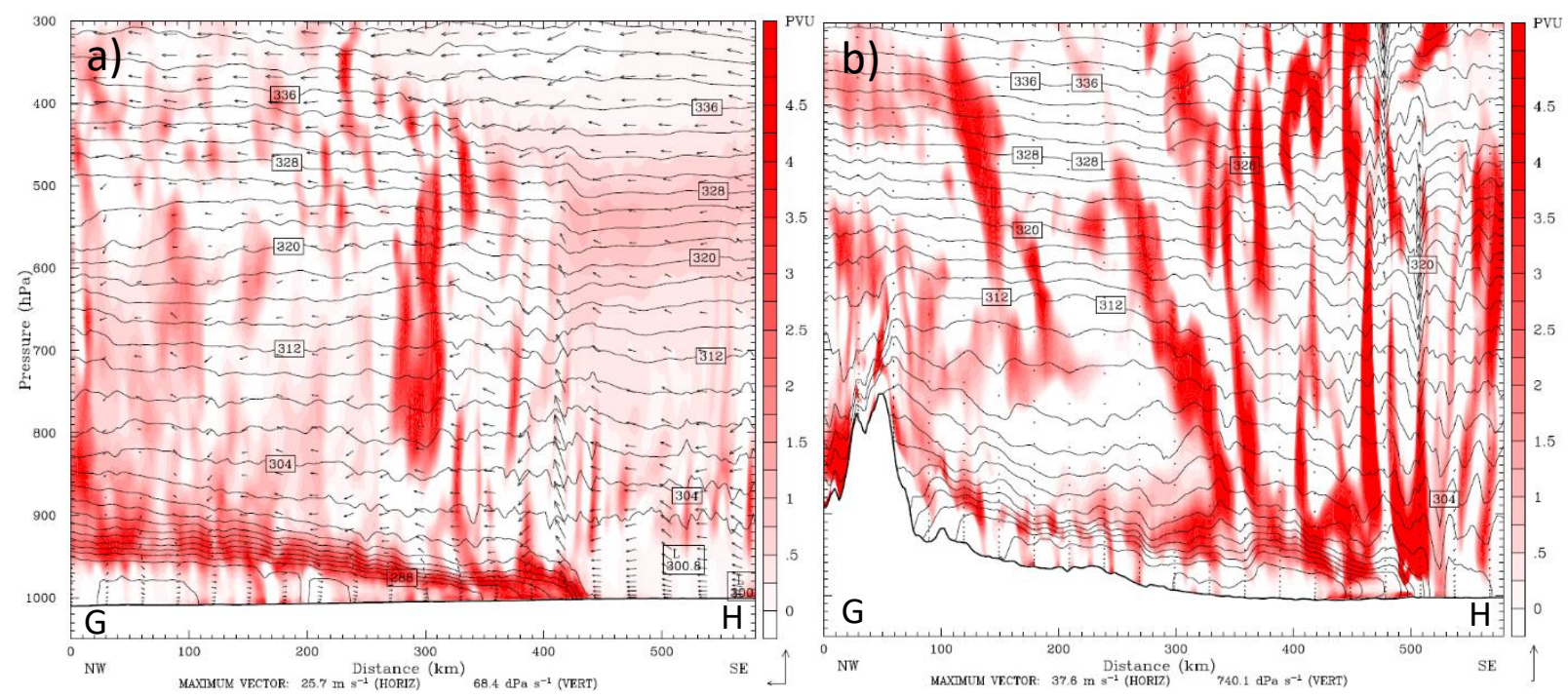

Figure 4.28. WRF cross sections for domain 2 at 1200 UTC 03 October 2015 for line segment GH (Fig. 4.27a) displaying potential vorticity (PVU; shaded red), potential temperature (K; contoured), and circulation vectors $\left[\mathrm{m} \mathrm{s}^{-1}, \mathrm{dPa} \mathrm{s}^{-1}\left(1 \mathrm{dPa} \mathrm{s}^{-1}=10^{-3} \mathrm{mb} \mathrm{s}^{-1}\right)\right]$ for the simulation with a) terrain removed and $\left.\mathrm{b}\right)$ terrain doubled.

terrain doubled (Fig. 4.28b). It was clear that the East Coast was on the cold side of a thermal boundary in both simulations, whether a mountain range was present or not. However, the boundary position was 100-km northwest of that in the doubled terrain simulation (Fig. 4.28b) when no orographic features existed (Fig. 4.28a). The potential temperature gradient appeared similar between the two simulations, except that an additional isentrope sloped to the surface in the doubled terrain simulation due to the slightly colder air pocket near the surface between the $350-\mathrm{km}$ and $450-\mathrm{km}$ distance marks (Fig. 4.28b). Isentropes also appeared steeper in slope near the boundary when comparing the doubled terrain simulation (Fig. 4.28b) to the simulation with no mountains (Fig. 4.28a), suggesting the potential for stronger lift near the front. 
Assessing the thermal boundary in the region of low-level frontogenesis in the control simulation (line segment CD; Fig. 4.8d) allowed for a direct comparison with the terrain-manipulated simulations. The propagation rates of the synoptic features were consistent in all simulations, making the analysis of the coastal front at the same time for all simulations an appropriate form of comparison. At 1200 UTC 03 October 2015, the boundary was located near the $10-\mathrm{km}$ distance in the simulation with no terrain (Fig. 4.29a), near the 37-km mark in the control simulation (Fig. 4.15b), and near the $68-\mathrm{km}$ mark with terrain doubled (Fig. 4.29b). Additionally, in the regions of the tightly packed isentropes, the slope to the surface began near $950 \mathrm{hPa}$ with no terrain (Fig. 4.29a), 925 $\mathrm{hPa}$ in the control simulation (Fig. 4.15b), and $900 \mathrm{hPa}$ with doubled terrain (Fig. 4.29b). The control simulation had the most tightly packed isentropes with the steepest slope.

The region of potential vorticity within the boundary stretched vertically to 800 $\mathrm{hPa}$ in the control simulation (Fig. 4.15b), but only to $950 \mathrm{hPa}$ with no mountains and $850 \mathrm{hPa}$ with higher mountains (Fig. 4.29b). The PV pattern is consistent with a welldefined thermally direct circulation in the control simulation (Fig. 4.15b), which was not as pronounced in the simulation with doubled terrain (Fig. 4.29b) and likely not present in the simulation with no terrain due to the decreased lift within the boundary (Fig. 4.29a). The strength of the vertical velocities within and just above the boundary in the doubled terrain simulation (Fig. 4.29b) are consistent with those in the control simulation (Fig. 4.11).

Deformation frontogenesis was weak in the thermal gradient near the surface with terrain removed (Fig. 4.29c) and similar in strength to the control simulation (Fig. 4.9d) when terrain was doubled (Fig. 4.29d). Divergence frontogenesis resulted in the same 

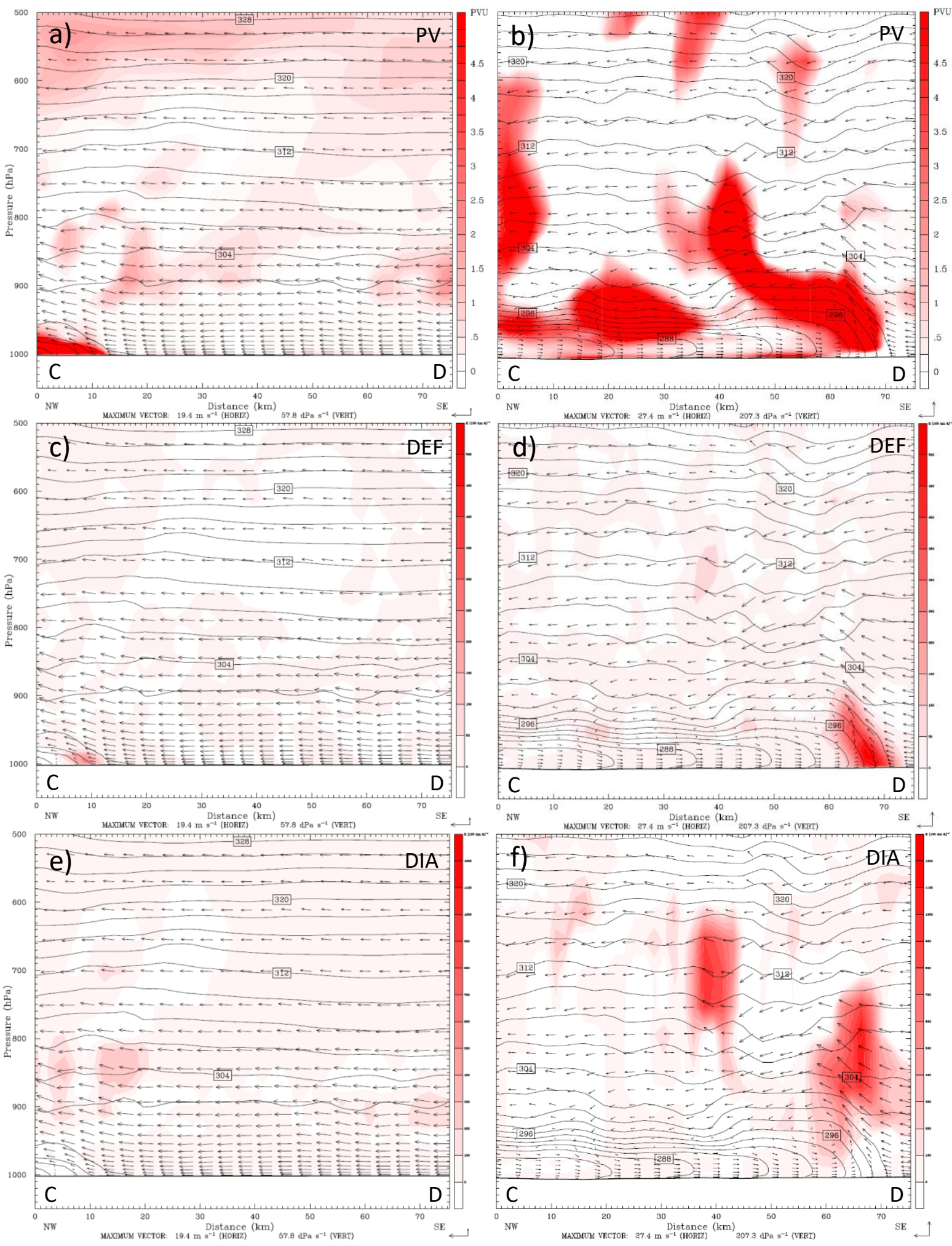

Figure 4.29. WRF domain 2 cross sections for line segment CD (Fig. 4.8d) at 1200 UTC 03 October 2015 displaying potential temperature ( $\mathrm{K}$; contoured) and circulation vectors $\left(\mathrm{m} \mathrm{s}^{-1}, \mathrm{dPa} \mathrm{s} \mathrm{s}^{-1}\right.$ ) with potential vorticity (PVU; shaded) for a) terrain removed and b) terrain doubled, deformation frontogenesis [K (100 $\mathrm{km} \mathrm{h}^{-1}$; shaded; positive red] for $\mathrm{c}$ ) terrain removed and d) terrain doubled, and diabatic frontogenesis [ $\mathrm{K}$ $\left(100 \mathrm{~km} \mathrm{~h}^{-1}\right.$; shaded; positive red] for e) terrain removed and f) terrain doubled. 
conclusions (not shown). Diabatic frontogenesis was nearly absent with no terrain (Fig. 4.29e) compared to the higher values seen in both the doubled terrain simulation (Fig. 4.29f) and the control simulation (Fig. 4.9f). The location of precipitation was further south in the simulation with no terrain, due to the synoptic environment, which made convection and the associated condensational heating absent in the region of the cross section. Contributing to the strength of the coastal front, diabatic frontogenesis stretched to nearly $600 \mathrm{hPa}$ in the control simulation (Fig. 4.9f) and only to $700 \mathrm{hPa}$ in the doubled terrain simulation (Fig. 4.29f). No matter the slight differences in the coastal front between the control simulation and the simulation with increased terrain height, both boundaries provided sufficient lift for convective initiation.

\subsubsection{Diabatic Processes}

The WRF control simulation of the 2015 South Carolina flood showed evidence

of diabatic frontogenesis in the mid-levels (Fig. 4.9f), suggesting latent heat release to be a factor in the maintenance of the coastal front and the thermally direct circulation. To investigate the role of diabatic/precipitation processes on the coastal front and subsequent lift, evaporation from precipitation was removed 6 hours prior to the start of intense convection in one WRF simulation, latent heating/cooling effects were turned off for the entirety of another simulation, and surface heat fluxes were turned off for the entirety of a third simulation. No evaporation resulted in a similar precipitation field as the control simulation (Fig. 4.1b), except totals were reduced by roughly 25\% (Fig. 4.30a). Removing latent heating shifted the precipitation field south off the coast of 


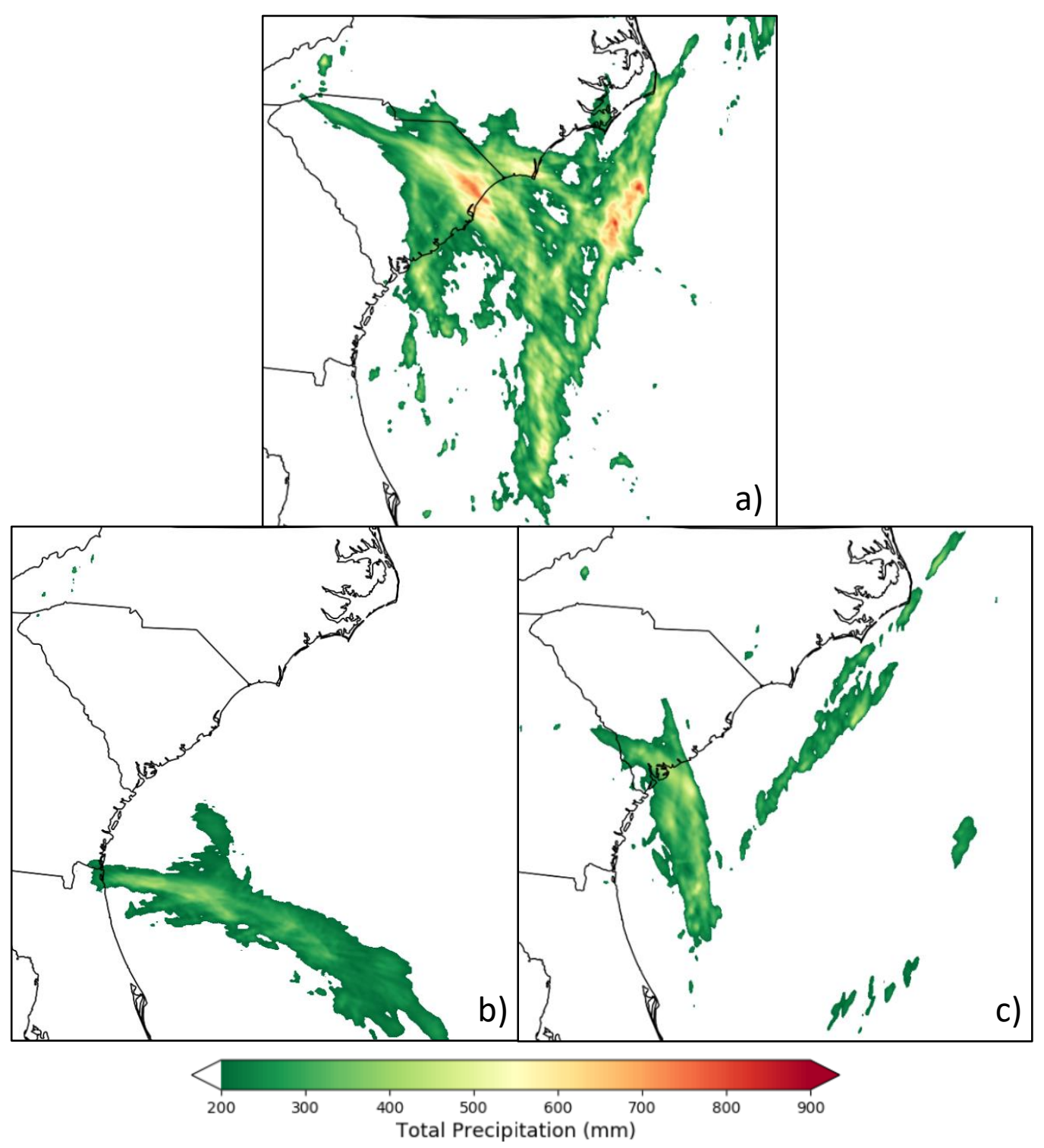

Figure 4.30. Total accumulated precipitation $(\mathrm{mm})$ from WRF domain 2 for the simulation with a) no evaporation from precipitation, b) no latent heating, and c) no surface heat fluxes.

Georgia/Florida and greatly reduced the accumulated total (Fig. 4.30b). Precipitation totals were also reduced when surface heat fluxes were removed, and the field shifted south with a north-south orientation stretching into southern South Carolina (Fig. 4.30c). 
From total accumulated precipitation alone, it appeared that all three processes had an impact on the heavy precipitation event.

On the synoptic scale, upper-level dynamics were nearly identical between the simulation with no evaporation (Fig. 4.31a) and that of the control simulation (Fig. 4.3a) for a 24-hour period ending at 1200 UTC 04 October 2015 (Fig. 4.31b). A significant difference was found between the control simulation and the simulation with no latent heating. Without condensational heating from precipitation, the upper-level jet was almost unrecognizable downstream of the trough with no associated upper-level divergence (Fig. 4.31c). A lack of latent heat release suggests little or no negative PV advection by the irrotational wind, which allowed for an increase in the progression of the upper-level low (Fig. 4.31d) consistent with the results of Marciano and Lackmann (2017). The upper-level jet over Georgia was weaker without surface heat fluxes, but upper-level divergence was still found in the right entrance region (Fig. 4.31e). The propagation of the upper-level low was consistent with the control simulation when no surface heat fluxes were involved, but the downstream jet was much weaker by 1200 UTC 04 October 2015 (Fig. 4.31f). The results from these simulations provide additional evidence for the conclusion that latent heat release was the main reason for the stagnant upper-level pattern, resulting in a positive feedback system that prolonged precipitation over South Carolina.

Also seen in the upper-level dynamics, the tropical cyclone remained near the Bahamas at 1200 UTC 03 October 2015 in a region of increased precipitable water even when no evaporation from precipitation was allowed (Fig. 4.32a). A moisture plume was directed towards South Carolina (Fig. 4.32a) like that found in the control simulation 


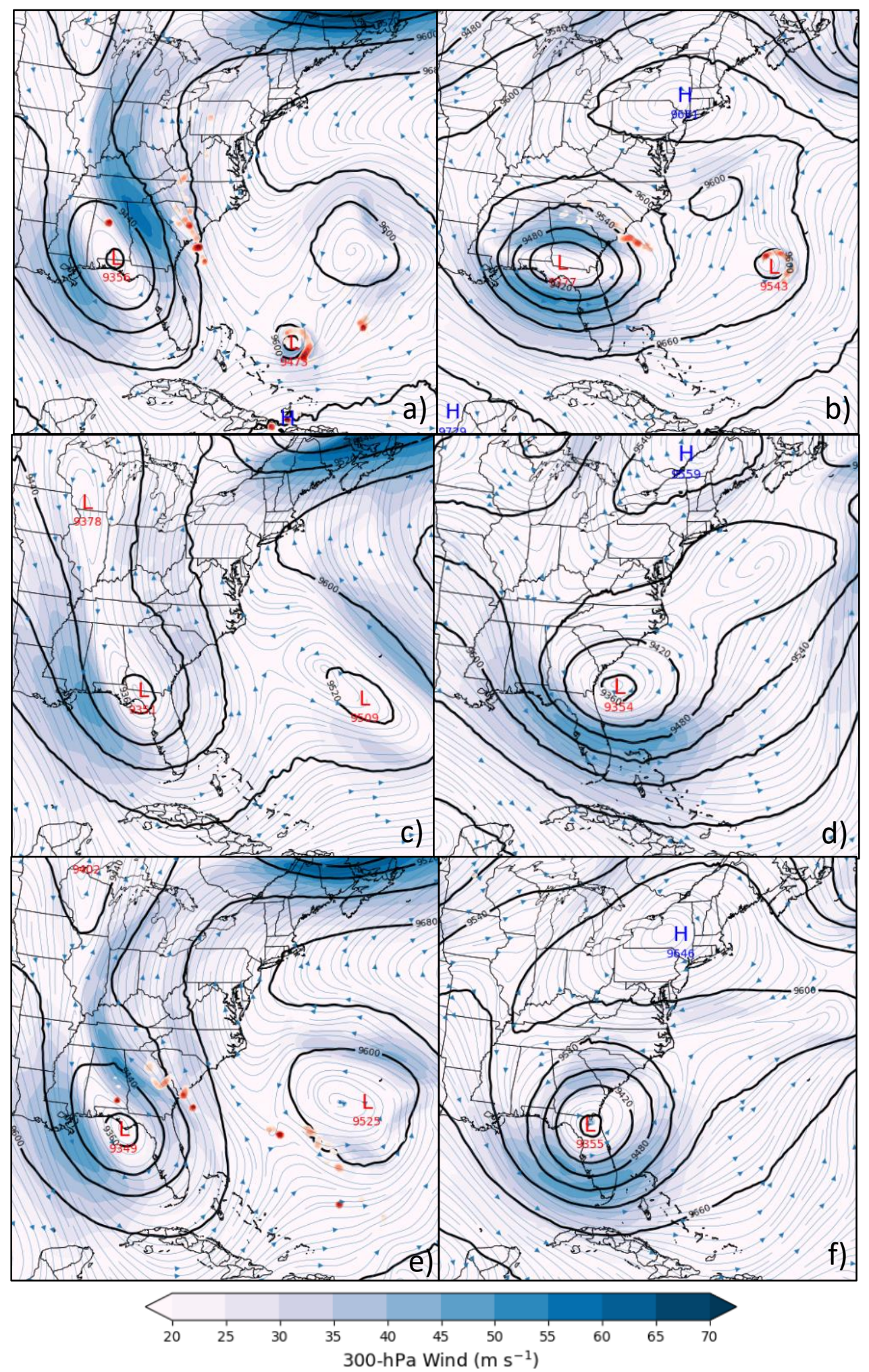

Figure 4.31. WRF domain 1300 -hPa height (gpm; thick contoured), wind ( $\mathrm{m} \mathrm{s}^{-1}$; shaded; thin streamlines), and divergence $\left(10^{-5} \mathrm{~s}^{-1}\right.$; contoured; positive red) for the simulation with no evaporation at a) 1200 UTC 03 October 2015 and b) 1200 UTC 04 October 2015, with no latent heating at c) 1200 UTC 03 October 2015 and d) 1200 UTC 04 October 2015, and with no surface heat fluxes at e) 1200 UTC 03 October 2015 and f) 1200 UTC 04 October 2015. 
(Fig. 4.4a). However, when latent heating was removed, the tropical cyclone was nonexistent, precipitable water values decreased east of the Bahamas, no deformation zone was present to form a north-south moisture plume, and the only moisture transport found was on the northeast side of the low pressure (Fig. 4.32b). The tropical cyclone and abundant moisture were also missing from the simulation with no surface heat fluxes (Fig. 4.32c), which was expected as most of the energy available to tropical storms comes from surface moisture and heat fluxes (Colle 2003). A moisture plume was still evident near South Carolina due to the position of the extratropical low (Fig. 4.32c), but the precipitable water values decreased drastically for that area by 04 October 2015 as the low propagated towards North Carolina and dry air approached from the south (not shown).

The location of increased precipitable water was consistent with the total accumulated precipitation fields of each simulation. The limited moisture in the simulations with no latent heating or surface heat fluxes aligned with the decreased precipitation totals, but the simulation with no evaporation also had decreased totals with nearly the same magnitude of precipitable water as the control simulation. The question remained as to how each of the three processes impacted the coastal front, as it was the main source of lift leading to an increase in precipitation intensity. The surface pattern for the simulation with no evaporation (Fig. 4.33a) appeared to be a duplicate of the control simulation (Fig. 4.6). Cold air damming, a strong temperature gradient near the coast $\left(15^{\circ} \mathrm{C} 100 \mathrm{~km}^{-1}\right)$, an inverted surface trough, and a wind shift suggested a coastal front (Fig. 4.33a). The simulations with no latent heating and no surface heat fluxes showed no signs of a frontal boundary. The removal of latent heating resulted in much warmer 


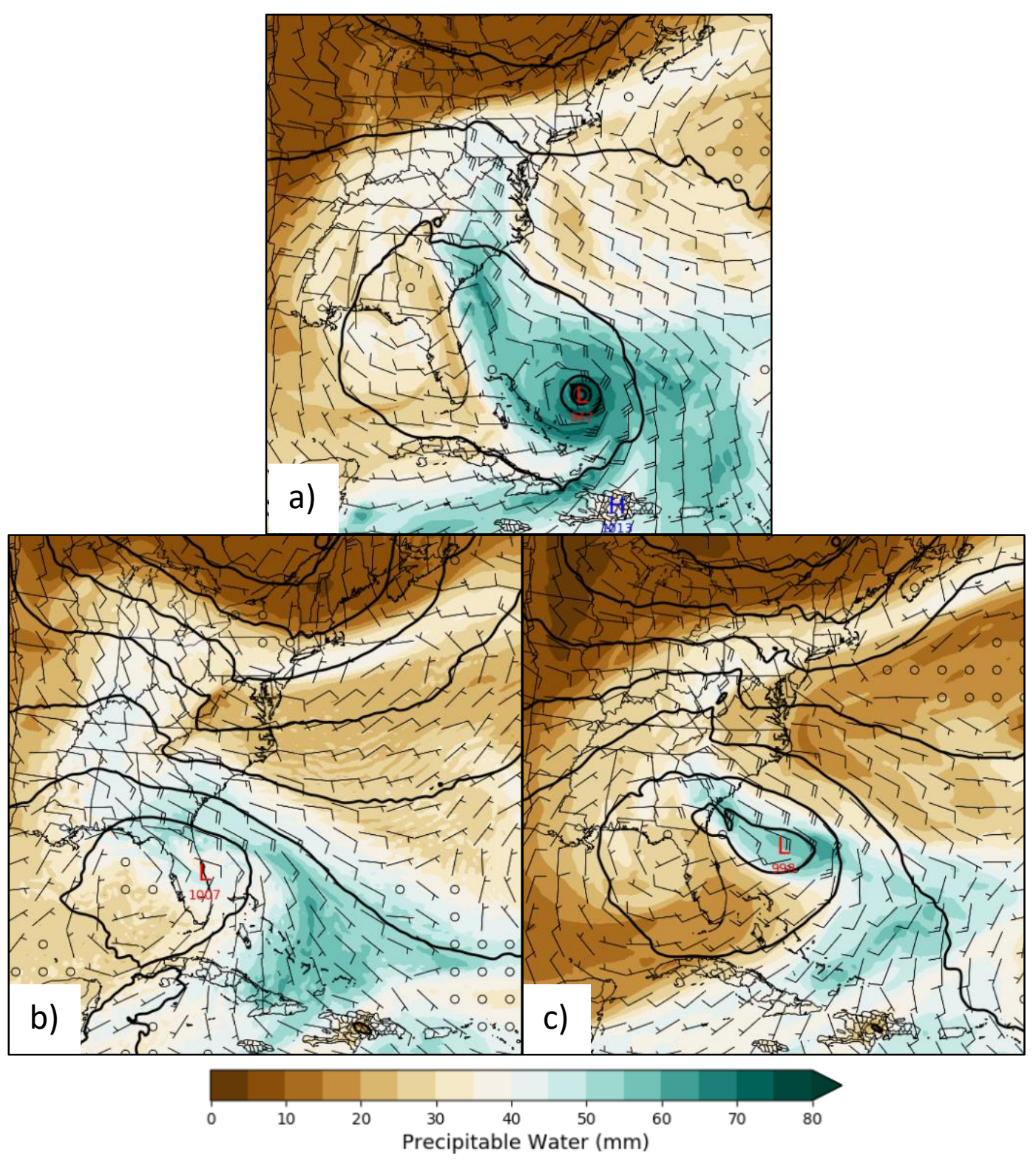

Figure 4.32. WRF results for domain 1 at 1200 UTC 03 October 2015 displaying precipitable water (mm; shaded), sea level pressure ( $\mathrm{hPa}$; contoured), and $850-\mathrm{hPa}$ wind $\left(\mathrm{m} \mathrm{s}^{-1}\right)$ for the simulation with a) no evaporation, b) no latent heating, and c) no surface heat fluxes.

surface temperatures $\left(\geq 32^{\circ} \mathrm{C}\right)$ off the coast of Florida, with no clear wind shift, no cold air damming, a very weak temperature gradient $\left(5^{\circ} \mathrm{C} 100 \mathrm{~km}^{-1}\right)$, and no amplified pattern in sea level pressure (Fig. 4.33b). The removal of surface heat fluxes also resulted in no 


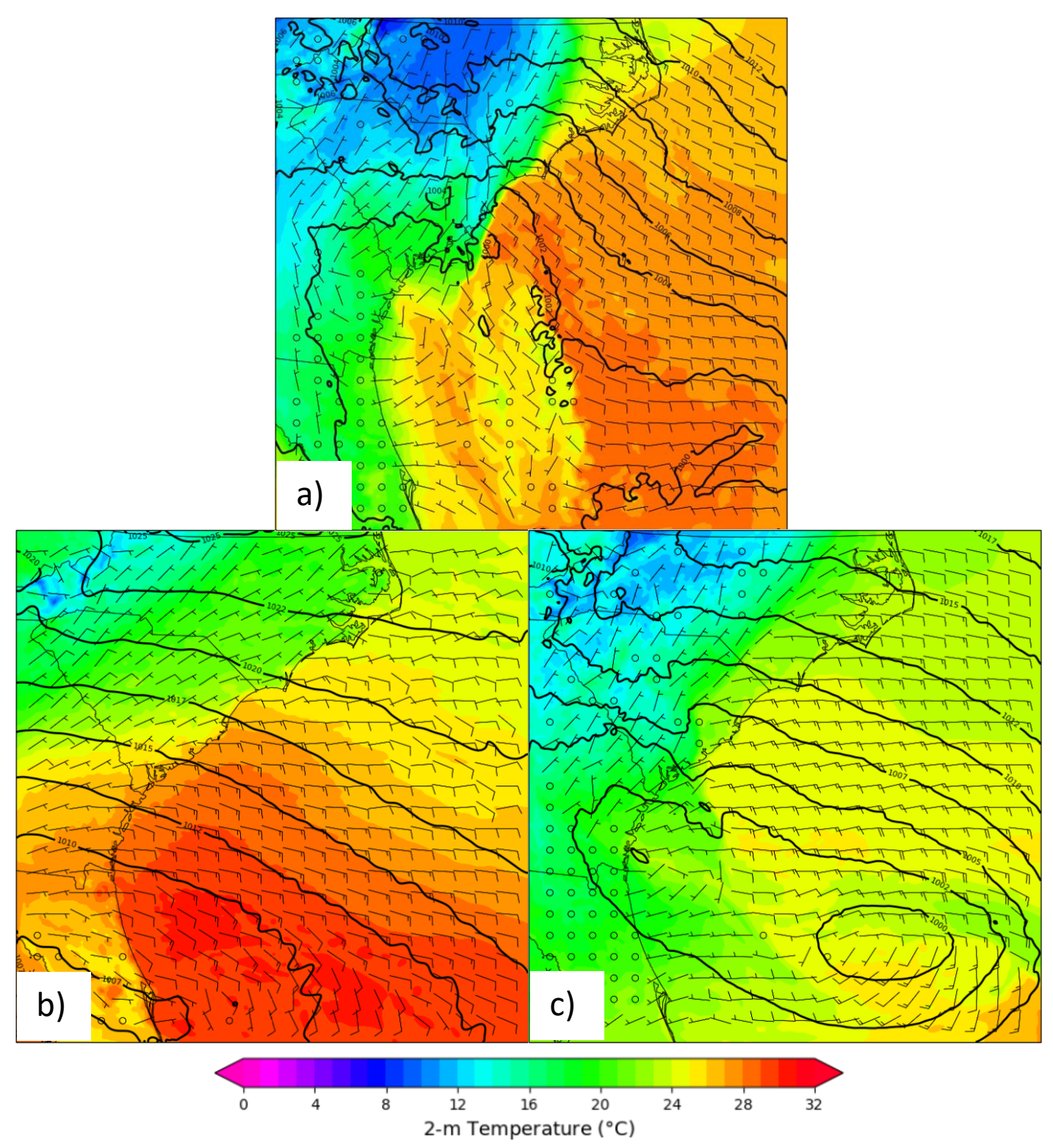

Figure 4.33. WRF results for domain 2 at 1200 UTC 03 October 2015 displaying sea level pressure (hPa; contoured), 2-m temperature ( ${ }^{\circ} \mathrm{C}$; shaded), and $10-\mathrm{m}$ wind $\left(\mathrm{m} \mathrm{s}^{-1}\right)$ for the simulation with a) no evaporation, b) no latent heating, and c) no surface heat fluxes.

coastal front, with weak cold air damming and much cooler surface temperatures (20$25^{\circ} \mathrm{C}$ ) over the Atlantic (Fig. 4.33c). 
Cross sections consistent with the location of precipitation in the control simulation (line segment CD) at 1200 UTC 03 October 2015 showed no frontogenesis off the coast of South Carolina in the simulations with no latent heating and no surface heat fluxes (not shown). A deeper investigation into the impacts of evaporation on the coastal front showed a northwest shift in the position of the boundary by about $10 \mathrm{~km}$ when evaporation from precipitation was removed (Fig. 4.34a). The PV pattern within the boundary was like that of the control simulation (Fig. 4.15b) near the surface, but it only extended to $970 \mathrm{hPa}$ consistent with the weaker frontal circulation and decreased lift (Fig. 4.34a). Divergence (Fig. 4.34b) and deformation frontogenesis (Fig. 4.34c) patterns were consistent with the control simulation near the surface within the boundary, while tilting was contributing to frontolysis in that area (Fig. 4.34d). Tilting and diabatic frontogenesis showed the same pattern with much weaker values in the mid-levels when evaporation was removed compared to the control simulation. Diabatic frontogenesis above the boundary only extended from 850 to $650 \mathrm{hPa}$ and was less than $10 \mathrm{~km}$ in horizontal extent (Fig. 4.34e). The control simulation had much stronger diabatic frontogenesis $\left[\sim 500 \mathrm{~K}(100 \mathrm{~km} \mathrm{~h})^{-1}\right]$ that even resulted in a downward sloping response in isentropes (Fig. 4.9f), which was not present after evaporation was removed.

Removing evaporation from precipitation also had an impact on relative humidity near South Carolina. Humidity values were consistent between the control simulation (Fig. 4.11) and the simulation with no evaporation (Fig. 4.34f), but only from the surface to $800 \mathrm{hPa}$ within and northwest of the frontal boundary. Overall, relative humidity in the region of the cross section was decreased when evaporation was not allowed (Fig. 4.34f). Additionally, the $\theta_{e}$ gradient was weaker near and above the frontal boundary when 

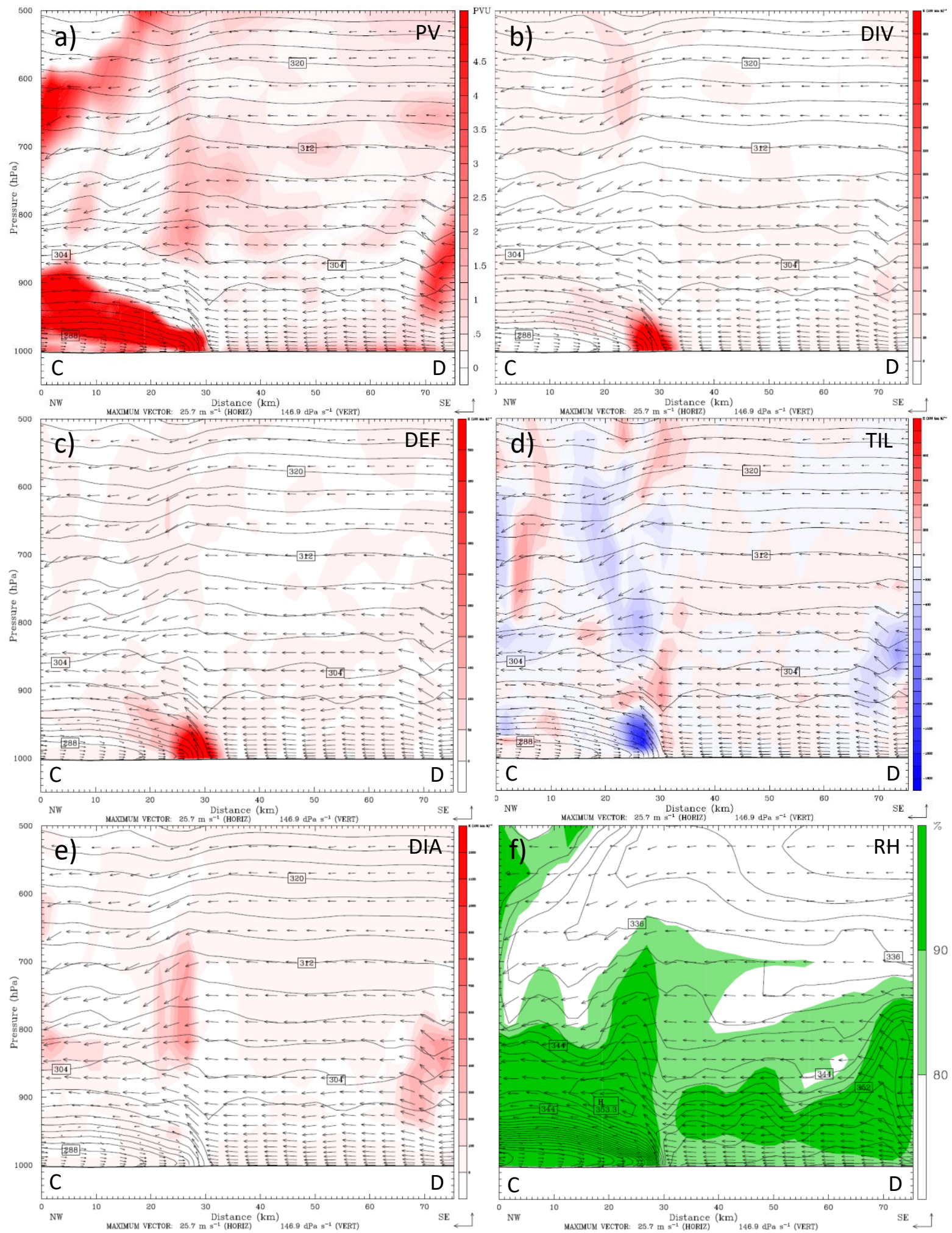

Figure 4.34. WRF domain 2 cross sections for the simulation with no evaporation at 1200 UTC 03 October 2015 for line segment CD (Fig. 4.8d) displaying potential temperature (K; contoured) and circulation vectors ( $\mathrm{m} \mathrm{s}^{-1}, \mathrm{dPa} \mathrm{s}^{-1}$ ) with a) potential vorticity (PVU; shaded) and b) divergence, c) deformation, d) tilting, and e) diabatic frontogenesis [ $\mathrm{K}(100 \mathrm{~km} \mathrm{~h})^{-1}$; shaded; positive red, negative blue], and $\left.\mathrm{f}\right) \mathrm{RH}$ (shaded) with $\theta_{e}$ (K; contoured). 
evaporation was removed (Fig. 4.34f). PI was found in association with RH values above $80 \%$ near $800 \mathrm{hPa}$ (Fig. 4.34f), located above the frontal boundary like that found in the control simulation (Fig. 4.11). The coastal front could have provided enough lift for the release of gravitational instability even when evaporation effects were removed, but the event would have been less intense than that in the control simulation as evident with the lower precipitation totals (Fig. 4.30a).

It was recognized that a complete removal of latent heating from a numerical model simulation requires no cumulus parameterization and no surface heat fluxes. The no latent heat simulation discussed above used no cumulus parameterization, but it did include surface heat fluxes. An additional simulation was completed with the removal of latent heating, surface heat fluxes, and no cumulus parameterization to ensure an accurate comparison between the simulations discussed. When all three effects were removed, upper-level dynamics, moisture, and sea level pressure patterns (not shown) were consistent with that of the shared simulation with no latent heating. Thus, latent heating had more of an impact on those environmental characteristics than surface heat fluxes. The biggest difference between the simulation with all three effects removed compared to the simulations discussed came in the magnitude of surface temperature. When latent heating and surface heat fluxes were both removed, the surface temperature off the coast of Florida was $\leq 28^{\circ} \mathrm{C}$ (not shown). That temperature pattern seemed to split the difference between that found in the simulation with no latent heating and that of the simulation with no surface heat fluxes. 


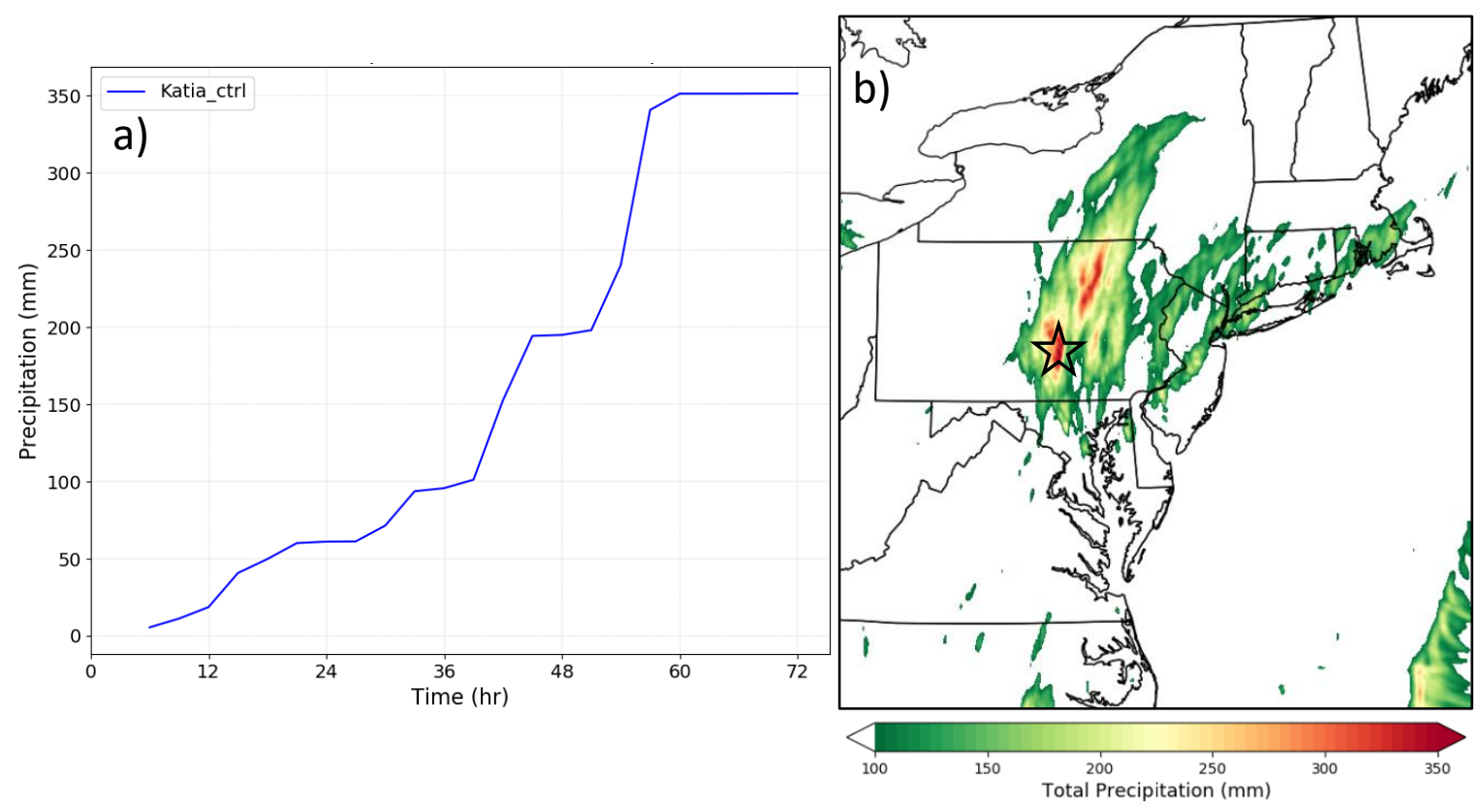

Figure 4.35. Precipitation (mm) for the 2011 flood event from 1200 UTC 06 September 2011 to 1200 UTC 09 September 2011 for domain 2 of the WRF control simulation a) at the location of maximum precipitation, plotted as accumulation with time and $b$ ) as the event total for the region. The transparent star represents the location of maximum precipitation.

\subsection{September 2011 Flood}

The WRF control simulation of the 2011 flood in Pennsylvania and New York showed the intense period of rainfall beginning near 0300 UTC 08 September and lasting until 0000 UTC 09 September 2011 (Fig. 4.35a). Two north-south oriented fields of precipitation occurred, both resulting in more than $300 \mathrm{~mm}$ of precipitation for the entirety of the event. The maximum precipitation received was $351.43 \mathrm{~mm}$ in southcentral Pennsylvania (Fig. 4.35b), with roughly $250 \mathrm{~mm}$ falling in a 24-hour period (Fig. 4.35a). 


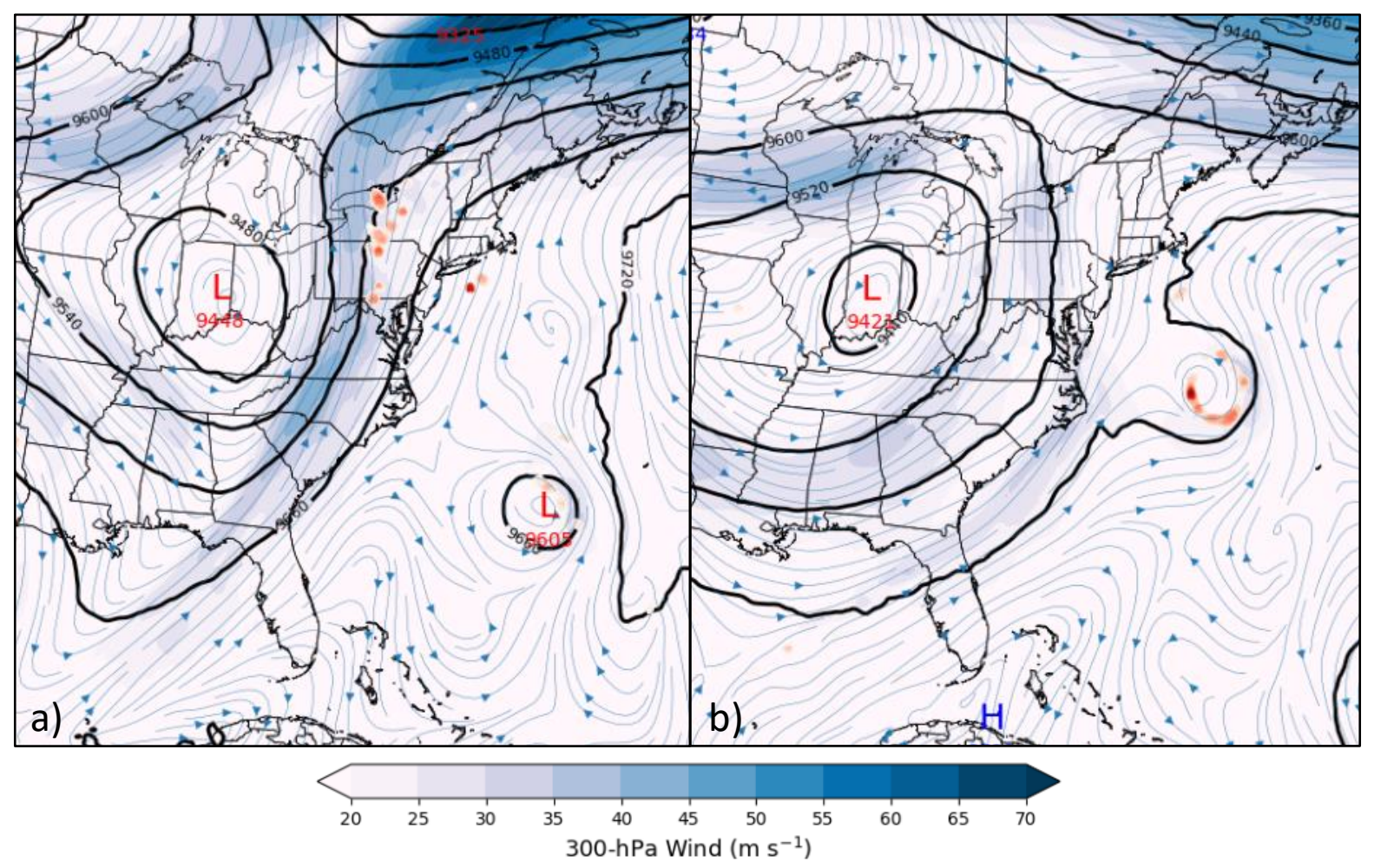

Figure 4.36. WRF domain 1 300-hPa height (gpm; thick contoured), wind ( $\mathrm{m} \mathrm{s}^{-1}$; shaded; thin streamlines), and divergence $\left(10^{-5} \mathrm{~s}^{-1}\right.$; contoured; positive red) at a) 0300 UTC 08 September 2011 and b) 0000 UTC 09 September 2011 of the control simulation.

\subsubsection{Synoptic Scale}

At the onset of intense precipitation near 0300 UTC 08 September 2011, an upper-level low was located over the Ohio River Valley (Fig. 4.36a). The upper-level jet associated with the low pressure was oriented north-south across Virginia and the Carolinas, but a secondary upper-level jet entrance region was located over western New York in association with a trough over eastern Canada. The jet interactions (i.e., jet coupling) led to upper-level divergence in central Pennsylvania (Fig. 4.36a), enhancing vertical velocities (Kastman et al. 2017). Due to a blocking ridge of high pressure over 


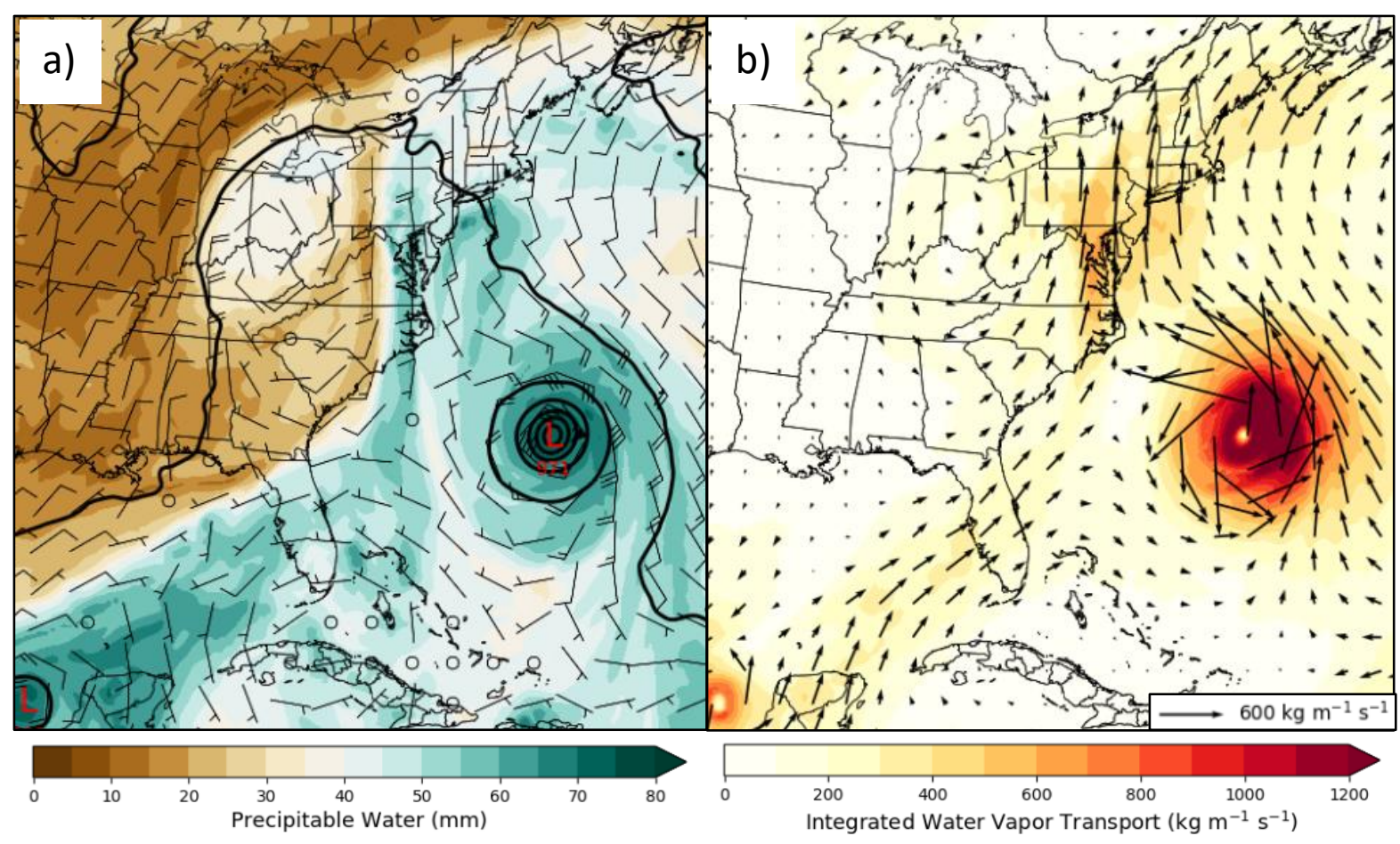

Figure 4.37. WRF results for domain 1 of the control simulation at 0300 UTC 08 September 2011 displaying a) precipitable water ( $\mathrm{mm}$; shaded), sea level pressure ( $\mathrm{hPa}$; contoured), 850-hPa wind ( $\mathrm{m} \mathrm{s}^{-1}$ ), and b) integrated water vapor transport (IVT; $\mathrm{kg} \mathrm{m}^{-1} \mathrm{~s}^{-1}$ ).

the western Atlantic, the upper-level low over the Ohio River Valley became cut off from the trough over eastern Canada and remained in the same location through the intense rain period (Fig. 4.36b). Hurricane Katia was able to travel north, embedded in the southerly flow between the upper-level low and the Atlantic ridge (Fig. 4.36b).

In association with the upper-level low, deep southerly flow persisted along the East Coast of the United States, creating a moisture plume from the North Carolina coast north through central Pennsylvania (Fig. 4.37a). A deformation zone between the 850$\mathrm{hPa}$ low pressure, associated with the upper low over the Ohio River Valley, and the tropical cyclone off the East Coast led to the narrow moisture plume (Fig. 4.37a) with 
concentrated IVT values around $600 \mathrm{~kg} \mathrm{~m}^{-1} \mathrm{~s}^{-1}$ from eastern Virginia north across central Pennsylvania (Fig. 4.37b). Moisture from the northwest outflow of the tropical cyclone was directed towards the region of heavy precipitation and may have been incorporated into the flow along the axis of dilatation directed at central Pennsylvania. However, the Gulf of Mexico and the Gulf Stream may have also contributed to increased moisture across central Pennsylvania, as the IVT pattern suggested (Fig. 4.37b). The moisture flow was aided by southwesterly wind at the surface associated with a surface low over the southeastern United States (not shown), a factor known to help organize a PRE into a linear structure (Cote 2007). 


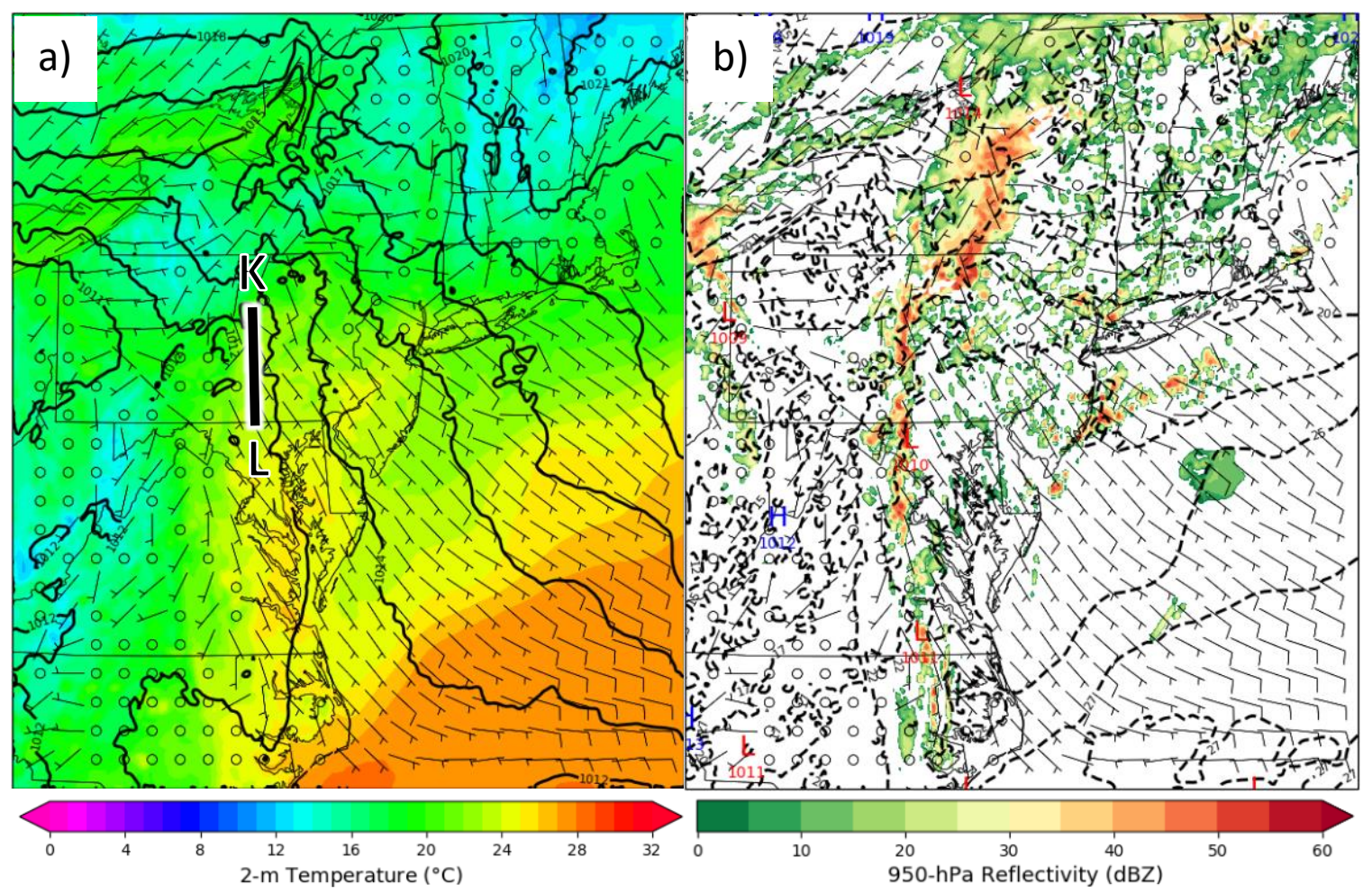

Figure 4.38. WRF results for domain 2 of the control simulation at 0300 UTC 08 September 2011 displaying 10-m wind $\left(\mathrm{m} \mathrm{s}^{-1}\right)$, a) sea level pressure ( $\mathrm{hPa}$; contoured), 2-m temperature $\left({ }^{\circ} \mathrm{C}\right.$; shaded), line segment $\mathrm{KL}, \mathrm{b}) 2-\mathrm{m}$ temperature $\left({ }^{\circ} \mathrm{C}\right.$; dashed), and 950-hPa reflectivity (dBZ; shaded).

\subsubsection{Mesoscale}

Surface conditions across central Pennsylvania made it difficult to find evidence of a frontal boundary. A wind shift and weak temperature gradient were present across the state, with south-southeasterly winds dominating eastern Pennsylvania and eastnortheasterly winds dominating western Pennsylvania (Fig. 4.38a), separated by the Appalachian Mountains. The precipitation field (Fig. 4.38b) was aligned with the moisture plume (Fig. 4.37a) and low-level jet (not shown). Based on the location of 


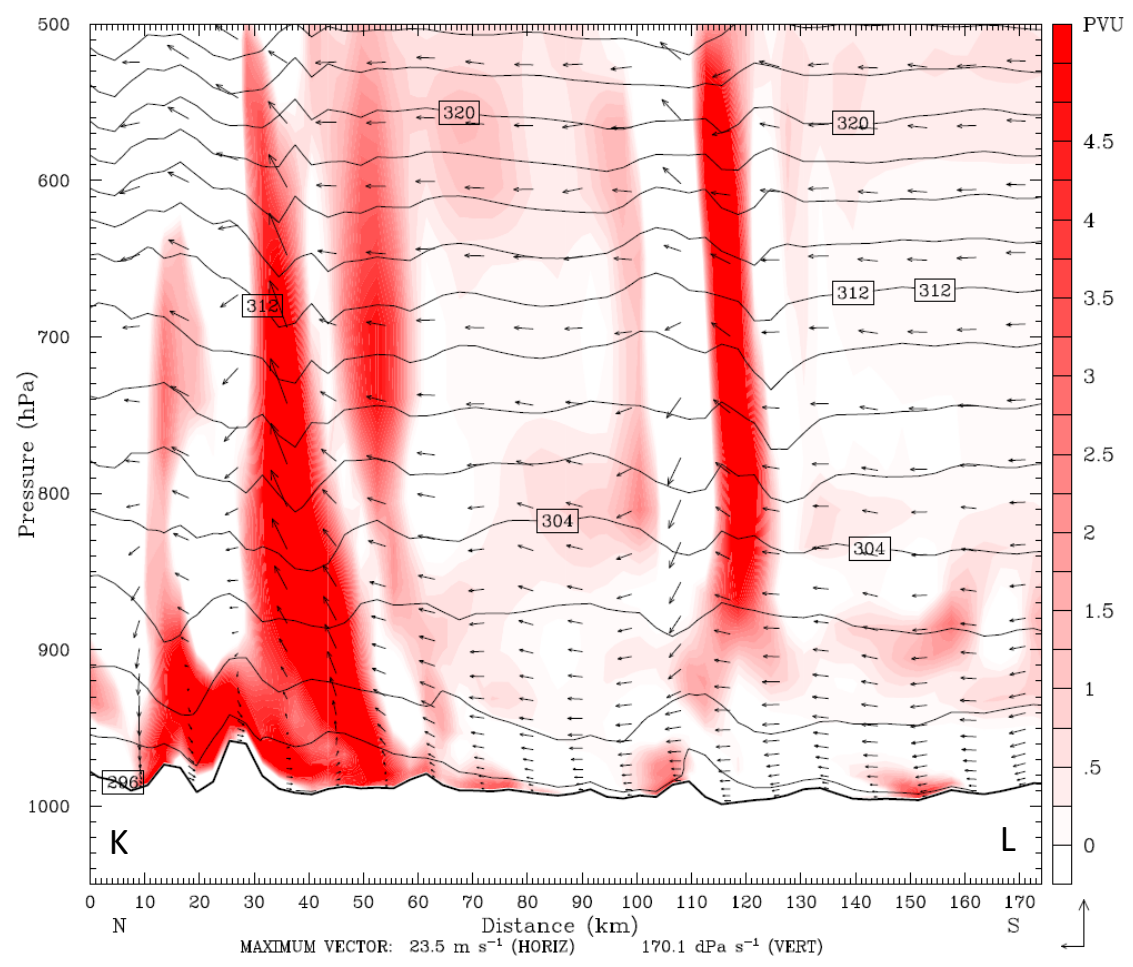

Figure 4.39. WRF domain 2 cross section from the control simulation at 0000 UTC 08 September 2011 for line segment KL (Fig. 4.38a) displaying potential vorticity (PVU; shaded red), potential temperature (K; contoured), and circulation vectors $\left[\mathrm{m} \mathrm{s}^{-1}, \mathrm{dPa} \mathrm{s}^{-1}\left(1 \mathrm{dPa} \mathrm{s}^{-1}=10^{-3} \mathrm{mb} \mathrm{s}^{-1}\right)\right]$.

precipitation and lack of a clear thermal boundary, it appeared the rain was initiated by orographic lift.

A cross section oriented along the line of precipitation showed a region of increased PV from the surface to $500 \mathrm{hPa}$, just south of an area of increased terrain near the onset of precipitation at 0000 UTC 08 September 2011 (Fig. 4.39). The region of PV was associated with increased lift along downward sloping isentropes (Fig. 4.39). Further investigation found negative tilting frontogenesis in the mid-levels associated with the increased PV, lift, and downward sloping isentropes (Fig. 4.40a), as expected. Weak deformation (Fig. 4.40b) and divergence (Fig. 4.40c) frontogenesis were located between 

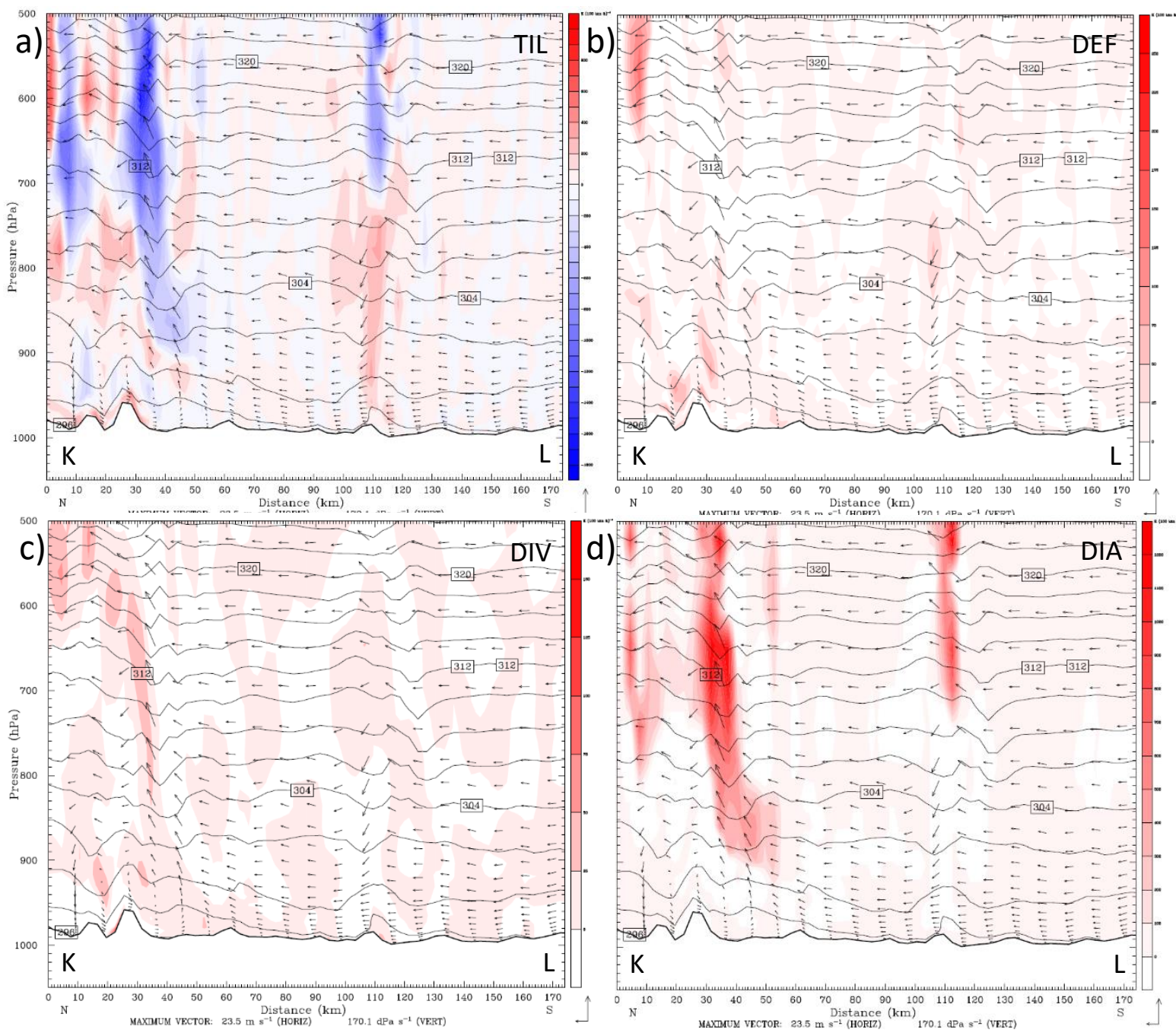

Figure 4.40. Cross sections KL (Fig. 4.38a) showing a) tilting, b) deformation, c) divergence, and d) diabatic frontogenesis [ $\mathrm{K}\left(100 \mathrm{~km} \mathrm{~h}^{-1}\right.$; shaded; positive red, negative blue], potential temperature ( $\mathrm{K}$; contoured), and circulation vectors $\left[\mathrm{m} \mathrm{s}^{-1}, \mathrm{dPa} \mathrm{s}^{-1}\left(1 \mathrm{dPa} \mathrm{s}^{-1}=10^{-3} \mathrm{mb} \mathrm{s}^{-1}\right)\right]$ at 0000 UTC 08 September 2011 from WRF domain 2 of the control simulation.

950-850 $\mathrm{hPa}$ near the mountain peak in association with the circulation. The strongest evidence of frontogenesis was found in the mid-levels in the region of strong ascent with positive divergence (Fig. 4.40c) and diabatic (Fig. 4.40d) frontogenesis. The sloping isentropes and diabatic frontogenesis were likely responses to mid-level latent heat release from convection in that location (not shown). 


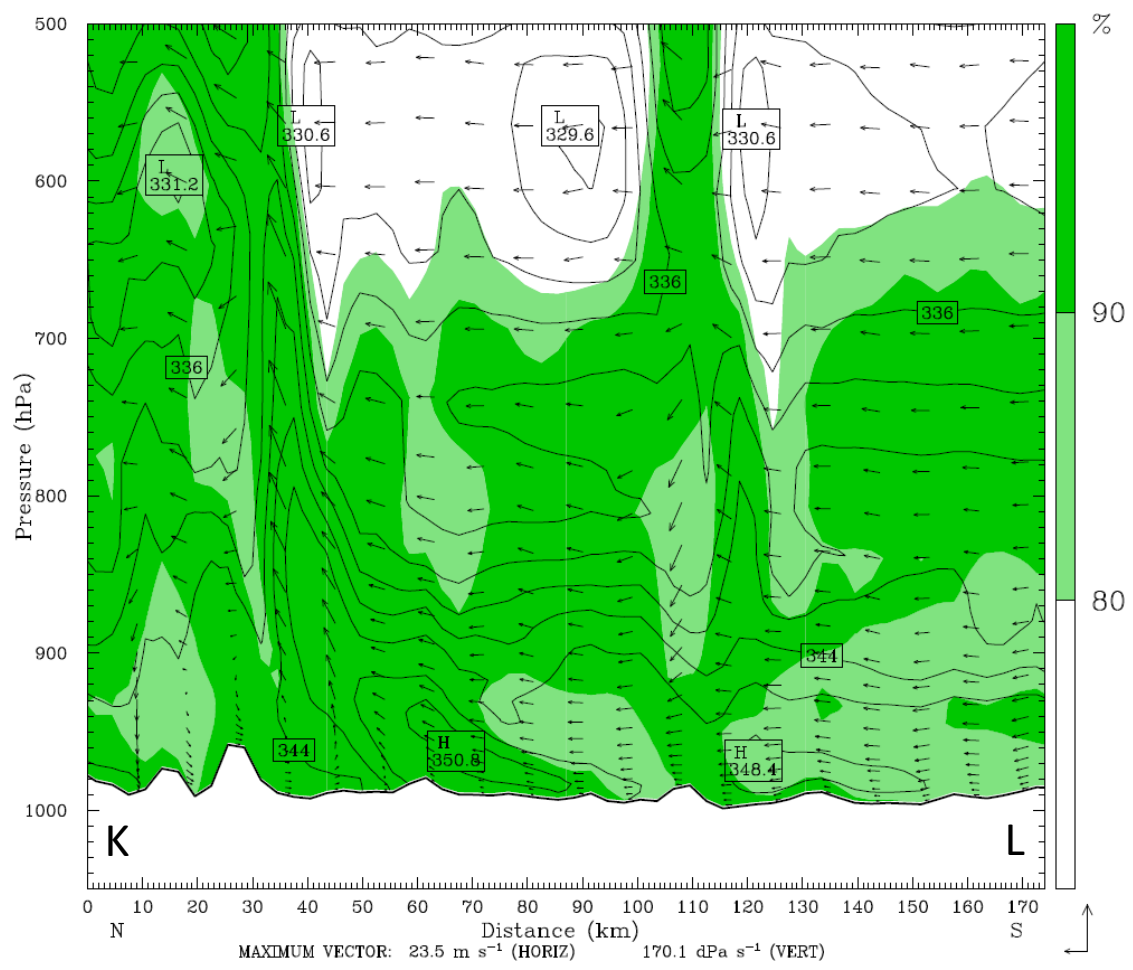

Figure 4.41. Cross sections KL (Fig. 4.38a) showing equivalent potential temperature (K; contoured), relative humidity (shaded), and circulation vectors $\left[\mathrm{m} \mathrm{s}^{-1}, \mathrm{dPa} \mathrm{s}^{-1}\left(1 \mathrm{dPa} \mathrm{s}^{-1}=10^{-3} \mathrm{mb} \mathrm{s}^{-1}\right)\right]$ at 0000 UTC 08 September 2011 from WRF domain 2 of the control simulation.

The heaviest precipitation at 0000 UTC 08 September was falling near point K (not shown), where frontogenesis dominated between 600-500 hPa (Fig. 4.40a, b, c, d). The same location was saturated, as was the region of strong ascent from the surface to $500 \mathrm{hPa}$ just south of the mountain peak (Fig. 4.41). Both areas had evidence of potential instability with decreasing $\theta_{e}$ with height (Fig. 4.41). Parcels were able to ascend along steep $\theta_{e}$ surfaces in a saturated environment, resulting in upright convection (not shown). Parcels lifted along the $\theta_{e}$ surfaces continued to lift and travel north in the mid-levels near point K (Fig. 4.41), making the initial lift of parcels just south of the mountain peak an aid to the convective initiation near point $\mathrm{K}$. 


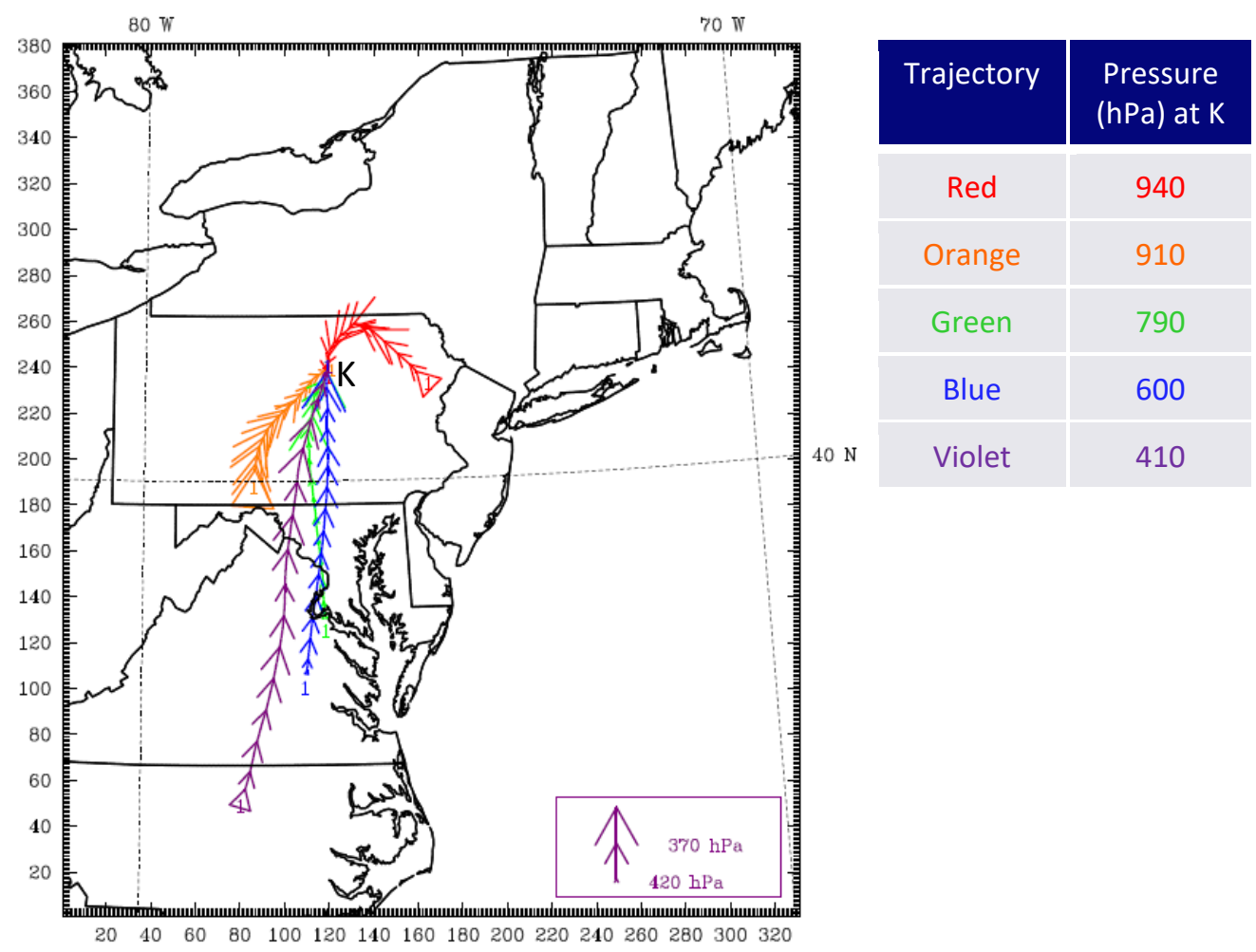

Figure 4.42. Parcel trajectories for the control simulation of the 2011 flood event beginning at 0600 UTC 08 September 2011 in the region of heavy rainfall, point K, and working backward six hours. The trajectories are colored by parcel pressure at point $\mathrm{K}$. The arrows on the trajectory represent the height of the parcel at that time, with larger arrows representing a higher level.

\subsubsection{Trajectories}

With a weak temperature gradient $\left(\sim 5^{\circ} \mathrm{C} 100 \mathrm{~km}^{-1}\right)$ along the mid-Atlantic coast (Fig. 4.38a), it was important to determine where parcels were traveling from to see if they may have crossed a thermal boundary before reaching central Pennsylvania. Parcel trajectories were calculated beginning at point K at 0600 UTC 08 September 2011, traveling backward six hours (Fig. 4.42). Five trajectories were initialized at varying heights above point $\mathrm{K}$ with initial pressures ranging from $940 \mathrm{hPa}$ (red) to $410 \mathrm{hPa}$ 


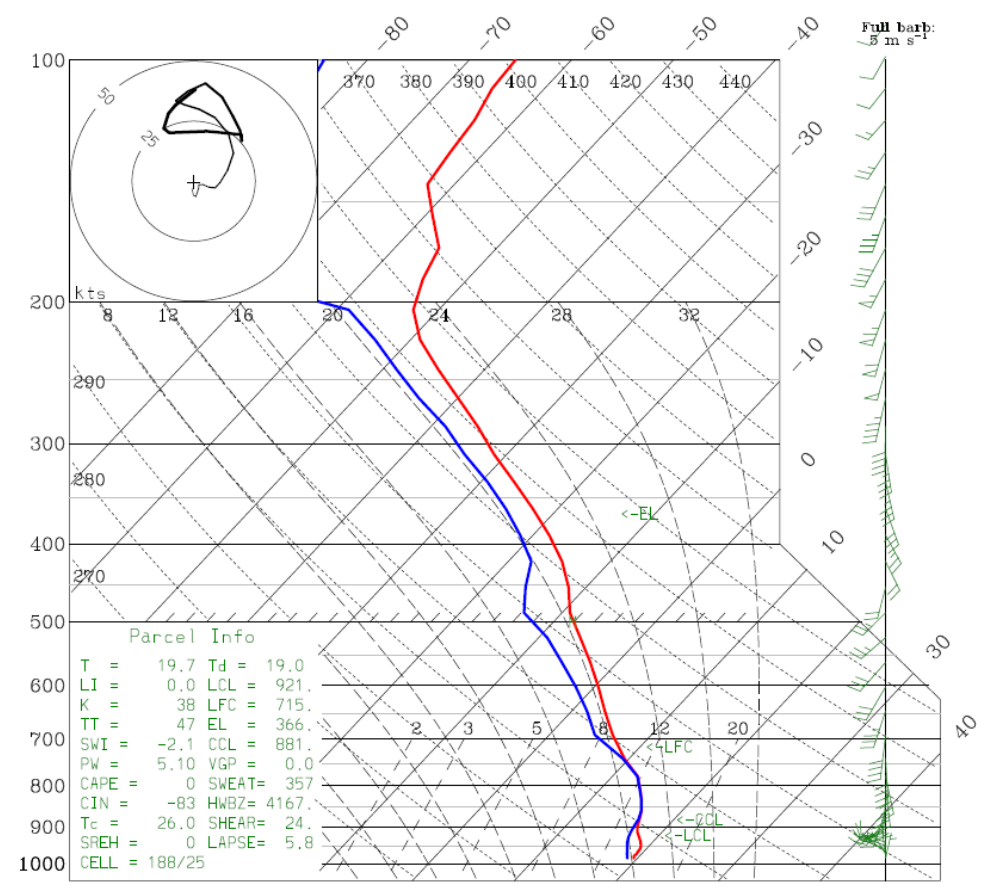

Figure 4.43. WRF sounding from domain 2 of the control simulation at point $\mathrm{K}$ (Fig. 4.42) showing temperature $\left({ }^{\circ} \mathrm{C}\right.$; red), dew point $\left({ }^{\circ} \mathrm{C}\right.$; blue), and wind $\left(\mathrm{m} \mathrm{s}^{-1}\right)$ at 0600 UTC 08 September 2011.

(violet), which correspond to five different eta levels in the WRF model at that time and location. The parcel closest to the surface appeared to originate in northeastern Pennsylvania and approached point K from the north (Fig. 4.42), but no lift was evident as it approached central Pennsylvania. The two parcels nearest to the surface and the parcel highest above the surface had descended as they approached K. Only the mid-level parcels (green and blue) had ascended near K (Fig. 4.42), which was consistent with the orographic lift seen at 0000 UTC 08 September 2011 (Fig. 4.39). Line segment KL was located along the mid-level trajectories from point $\mathrm{K}$ to roughly the southern Pennsylvania border (Fig. 4.38a). Cross section results along KL suggested that the parcels did not cross a frontal boundary as they approached central Pennsylvania (Fig. 
4.39). Additionally, the mid-level parcel trajectories originated almost due south of the area of precipitation and traveled along a line corresponding to the location of the moisture plume (Fig. 4.37b), which suggested they were nearly saturated as they lifted on approach to point $\mathrm{K}$.

A sounding at point K at 0600 UTC 08 September 2011 (Fig. 4.43), the time at which the parcels had reached point K (Fig. 4.42) and intense precipitation was occurring, was consistent with the assumptions made from the trajectories. Wind near the surface was northerly, while westerly winds dominated below $900 \mathrm{hPa}$ (Fig. 4.43). The atmosphere was stable below $900 \mathrm{hPa}$ with drier air, while the environment was saturated between 900 and $750 \mathrm{hPa}$, with southerly winds around $30 \mathrm{~m} \mathrm{~s}^{-1}$. With convection initiating in the mid-levels at point $\mathrm{K}$, and evidence of a dry, stable layer near the surface, it is possible the convection was elevated in nature. However, no clear frontal boundary was present near the surface, suggesting the surface stability and forcing for ascent to be induced by orography.

\subsubsection{Role of the Tropical Cyclone}

As with the 2015 South Carolina flood, the precipitation field in this case had two axes of precipitation across central Pennsylvania in September 2011 (Fig. 4.35b). When the tropical cyclone was removed from the WRF initial conditions, the precipitation field became confined to one main axis across Pennsylvania (Fig. 4.44a), consistent with the results of the 2015 event. The maximum precipitation for the event increased to 389.88 mm when Hurricane Katia was removed. While the environment in the western Atlantic 


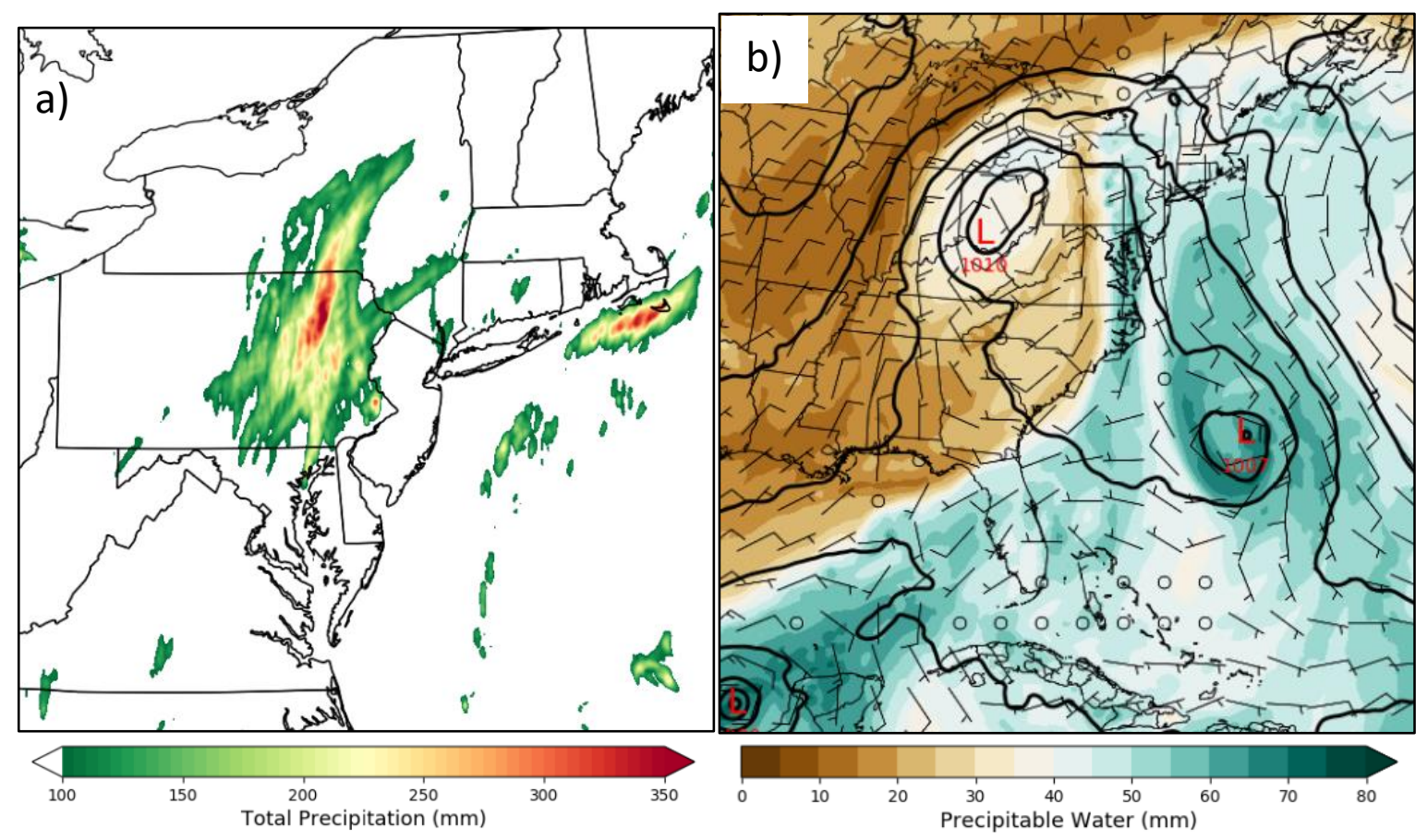

Figure 4.44. WRF results of the simulation with the tropical cyclone removed displaying a) total accumulated precipitation $(\mathrm{mm})$ for domain 2 and b) precipitable water ( $\mathrm{mm}$; shaded), sea level pressure (hPa; contoured), and 850-hPa wind $\left(\mathrm{m} \mathrm{s}^{-1}\right)$ for domain 1 at 0300 UTC 08 September 2011.

was still ideal for tropical cyclone development, a low formed in the area of Hurricane Katia (Fig. 4.44b).

The moisture pattern remained similar to the control simulation, but the deformation zone was not as defined (Fig. 4.44b). As with the 2015 flood, the tropical cyclone vortex was able to manipulate the angle of the axis of dilatation as it moved north, shifting the axis of concentrated moisture and heaviest precipitation, creating multiple elongated areas of rainfall totals above $300 \mathrm{~mm}$. With the removal of the tropical cyclone vortex, the moisture plume remained consistent and allowed precipitation to fall along the same axis for the entire event, increasing the maximum rainfall. In the 2011 


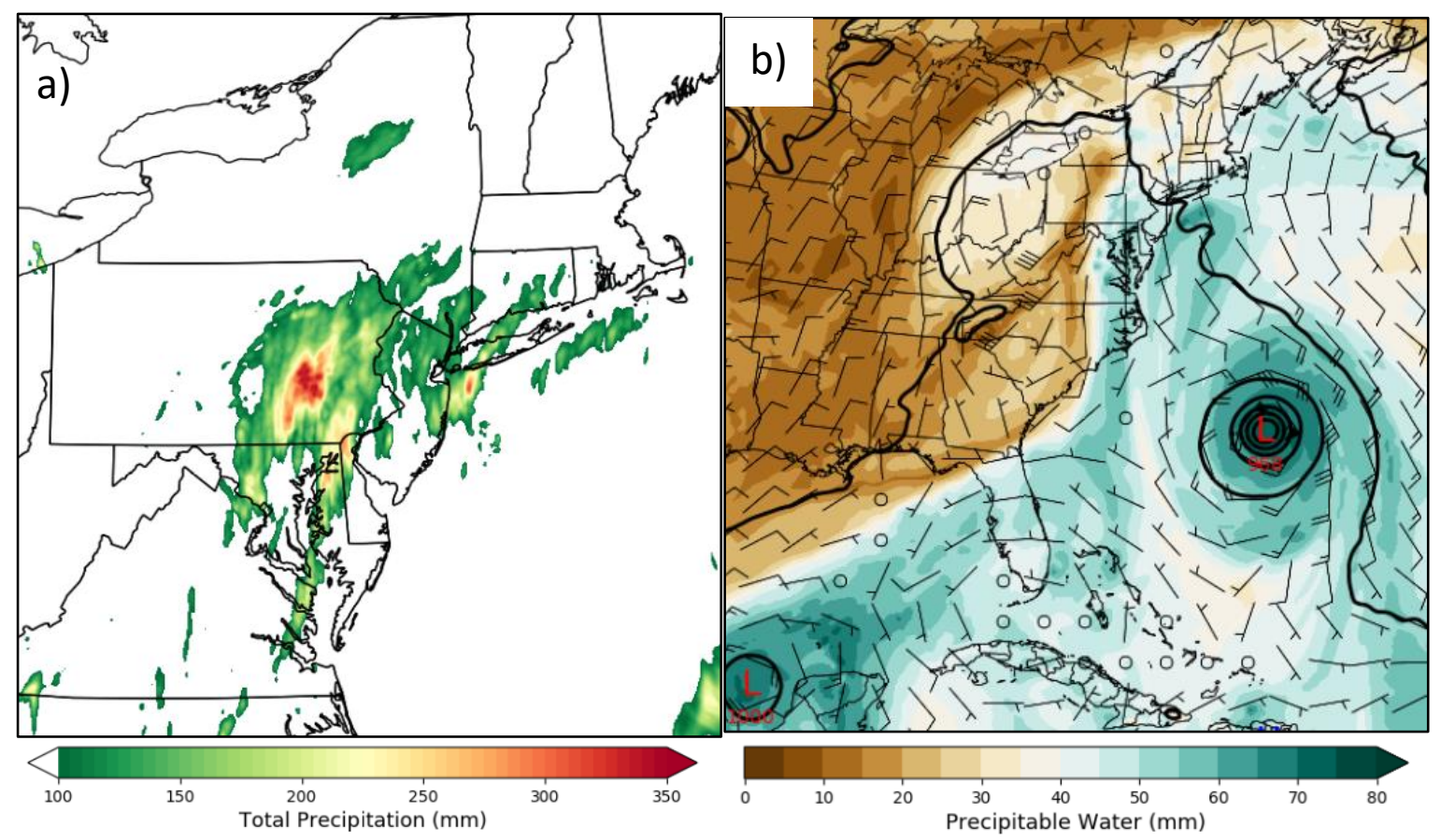

Figure 4.45. WRF results of the simulation with terrain height doubled displaying a) total accumulated precipitation $(\mathrm{mm})$ for domain 2 and b) precipitable water ( $\mathrm{mm}$; shaded), sea level pressure ( $\mathrm{hPa}$; contoured), and 850-hPa wind $\left(\mathrm{m} \mathrm{s}^{-1}\right)$ for domain 1 at 0300 UTC 08 September 2011.

event, Hurricane Katia appeared to have no impact on the extratropical low or jet streaks over the eastern United States.

\subsubsection{Terrain Influence}

Doubling the height of terrain in the WRF simulation of the 2011 event resulted in similar precipitation totals, with a slightly different location of maximum precipitation (Fig. 4.45a). The heaviest precipitation fell over southeastern Pennsylvania, suggesting the increased terrain height to have an impact on the moisture plume. The increased terrain allowed for drier air over central Pennsylvania, with the region of increased 


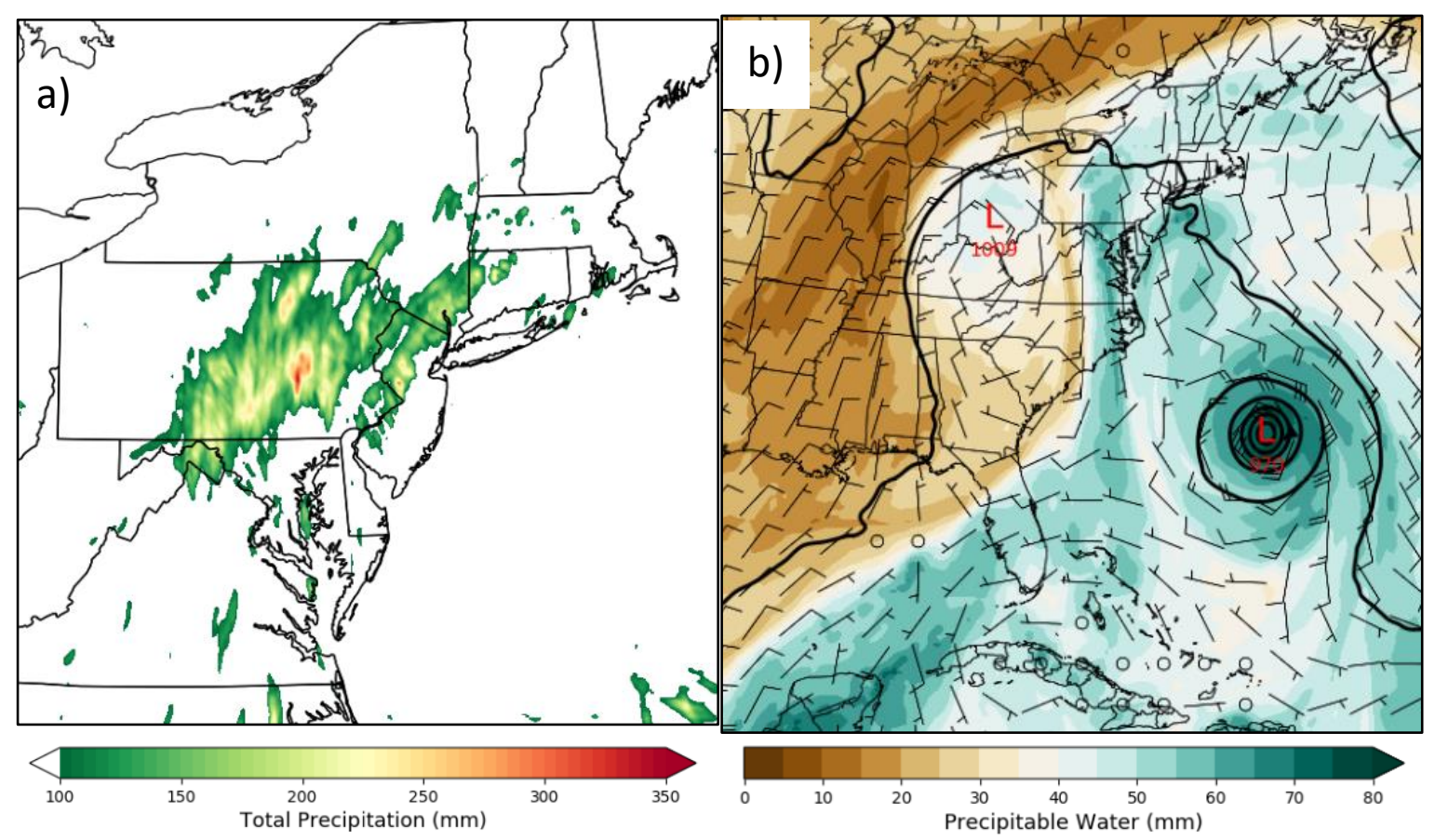

Figure 4.46. WRF results of the simulation with terrain removed displaying a) total accumulated precipitation $(\mathrm{mm})$ for domain 2 and b) precipitable water $(\mathrm{mm}$; shaded), sea level pressure ( $\mathrm{hPa}$; contoured), and 850-hPa wind $\left(\mathrm{m} \mathrm{s}^{-1}\right)$ for domain 1 at 0300 UTC 08 September 2011.

precipitable water confined to southeastern Pennsylvania (Fig. 4.45b) due to the terrain. Doubling terrain appeared to have no impact on the upper-level pattern, tropical cyclone track, or surface temperature gradient near the coast (not shown).

Removing terrain resulted in a broader region of precipitation with much lower totals overall and only one small area in eastern Pennsylvania with a maximum above 300 mm (Fig. 4.46a). Precipitable water values increased slightly over central Pennsylvania (Fig. 4.46b). The 850-hPa low pressure and its associated flow was uninterrupted by mountains, allowing for an increase in precipitable water over western 


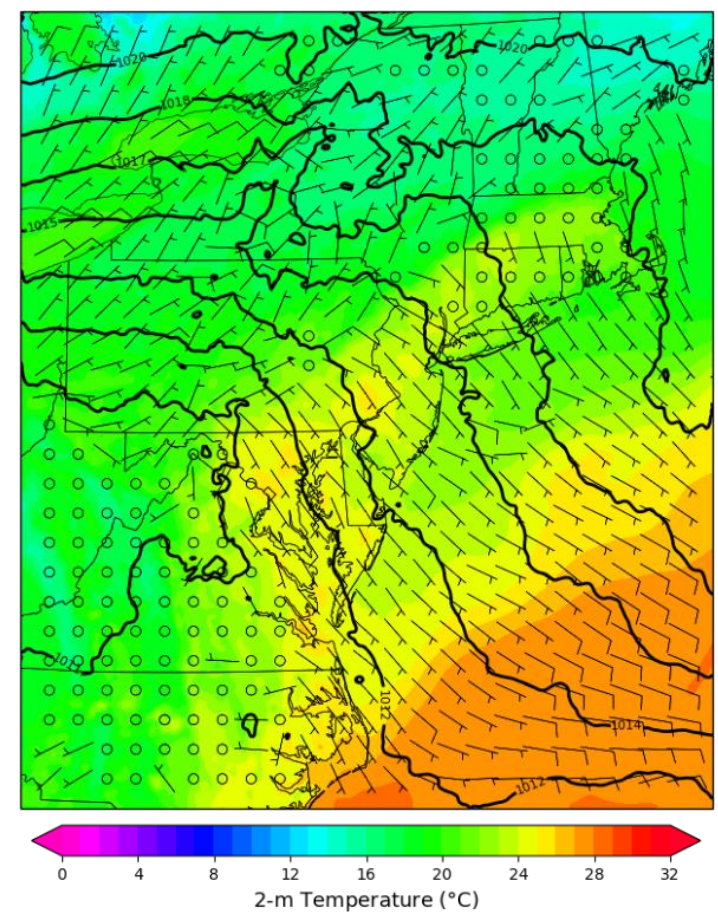

Figure 4.47. WRF results for domain 2 of the simulation with terrain removed at 0300 UTC 08 September 2011 displaying sea level pressure ( $\mathrm{hPa}$; contoured), 10-m wind $\left(\mathrm{m} \mathrm{s}^{-1}\right)$, and 2-m temperature $\left({ }^{\circ} \mathrm{C}\right.$; shaded).

Pennsylvania (Fig. 4.46b). The upper-level pattern remained consistent without terrain, allowing for upper-level divergence over Pennsylvania (not shown).

At the surface, a wind shift was evident across southeastern Pennsylvania in association with a temperature gradient of only about $5^{\circ} \mathrm{C} 100 \mathrm{~km}^{-1}$ (Fig. 4.47). The location of the wind shift aligns with the southeastern edge of the precipitation field (Fig. 4.46a), suggesting a weak frontal boundary to be the source of lift for the convection. No further analysis was completed to determine the role of the weak boundary, but it is likely the mechanisms for convective initiation were dependent on the Appalachian Mountains. 


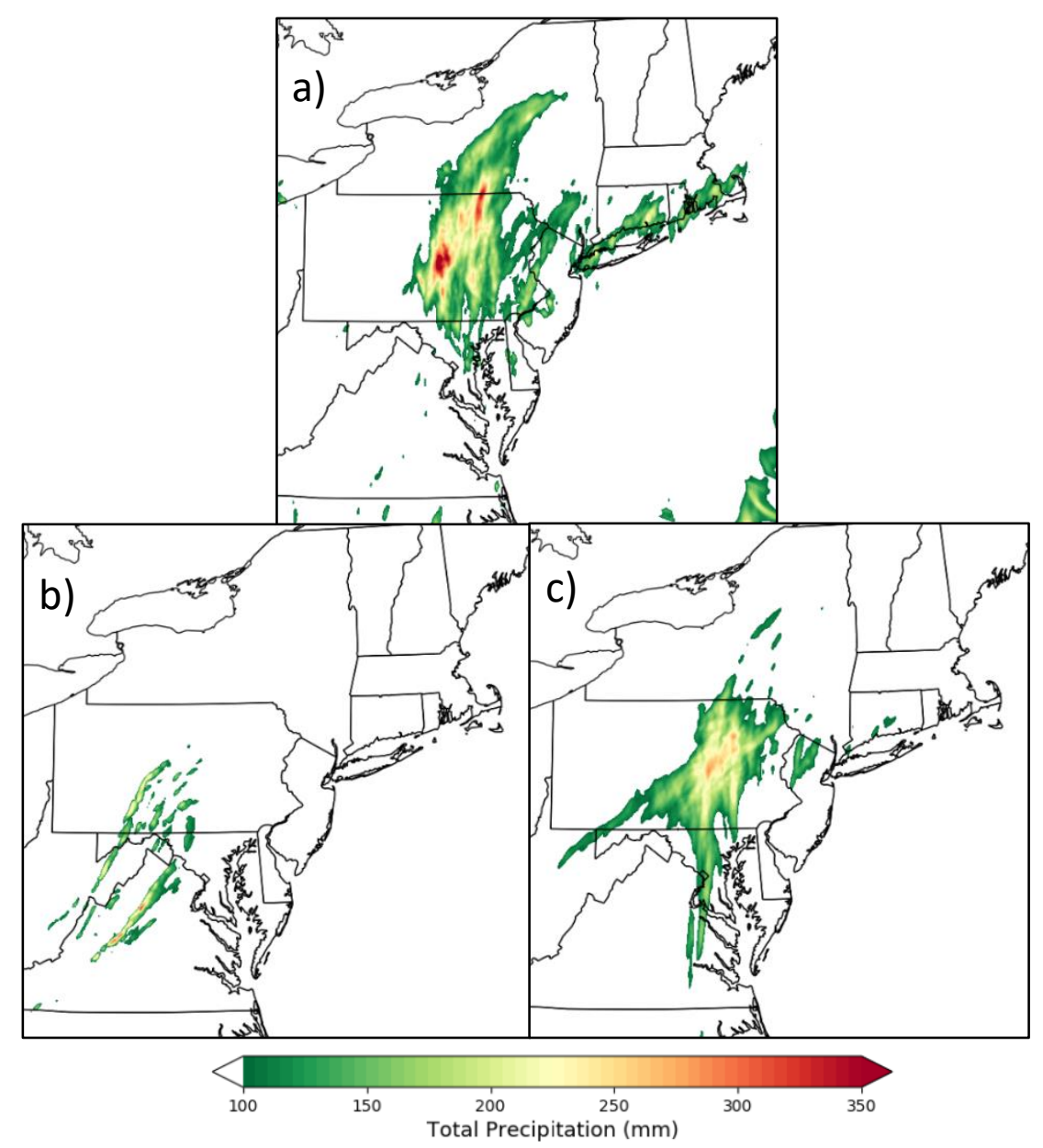

Figure 4.48. Total accumulated precipitation $(\mathrm{mm})$ from WRF domain 2 for the simulation with a) no evaporation from precipitation, b) no latent heating, and c) no surface heat fluxes.

\subsubsection{Diabatic Processes}

Removing evaporation from precipitation in the WRF simulation resulted in an increase of precipitation along two axes in central Pennsylvania (Fig. 4.48a). The maximum of precipitation for the event occurred in roughly the same location as that of the control simulation, except the maximum increased to $404.79 \mathrm{~mm}$. No impacts were found to the moisture plume or either cyclone when evaporative cooling was removed. 


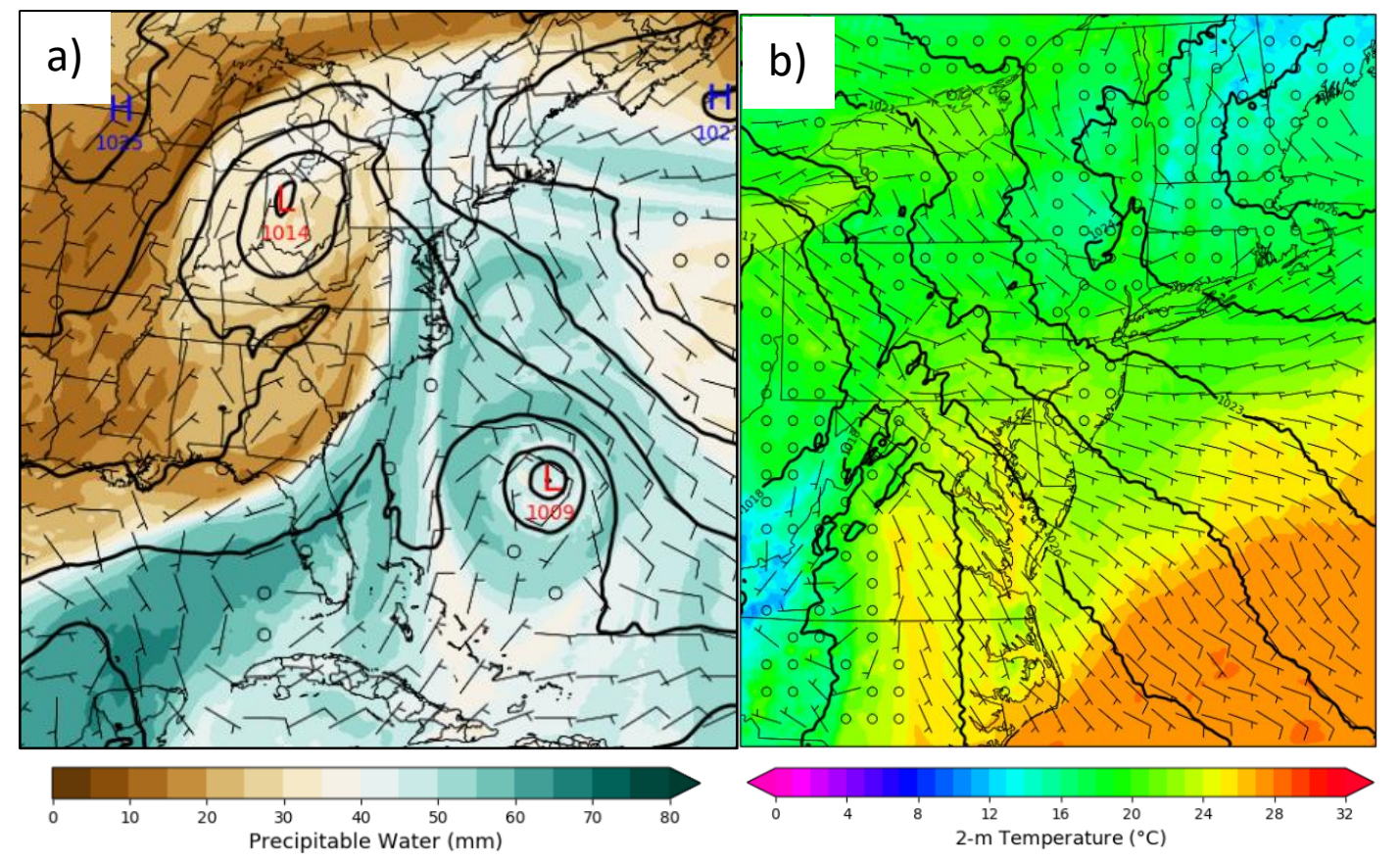

Figure 4.49. WRF results for the simulation with no latent heating at 0300 UTC 08 September 2011 displaying a) precipitable water ( $\mathrm{mm}$; shaded), sea level pressure ( $\mathrm{hPa}$; contoured), and 850-hPa wind (m $\mathrm{s}^{-1}$ ) from domain 1 and $\mathrm{b}$ ) sea level pressure ( $\mathrm{hPa}$; contoured), 10-m wind ( $\left.\mathrm{m} \mathrm{s}^{-1}\right)$, and 2-m temperature $\left({ }^{\circ} \mathrm{C}\right.$; shaded) from domain 2.

The increase in precipitation totals without a change in the large-scale atmospheric features suggested latent heating to have played a major role in the intensity of the convection.

To further support the theory of latent heating being the main contributor to convective intensity, the removal of latent heating from the WRF simulation resulted in very little precipitation over Pennsylvania (Fig. 4.48b). The only precipitation that fell was caused by the orographic lift of saturated air, with the precipitation field mirroring the orientation of the mountain range (Fig. 4.48b). Removing surface heat fluxes also inhibited precipitation totals over central Pennsylvania (Fig. 4.48c). 


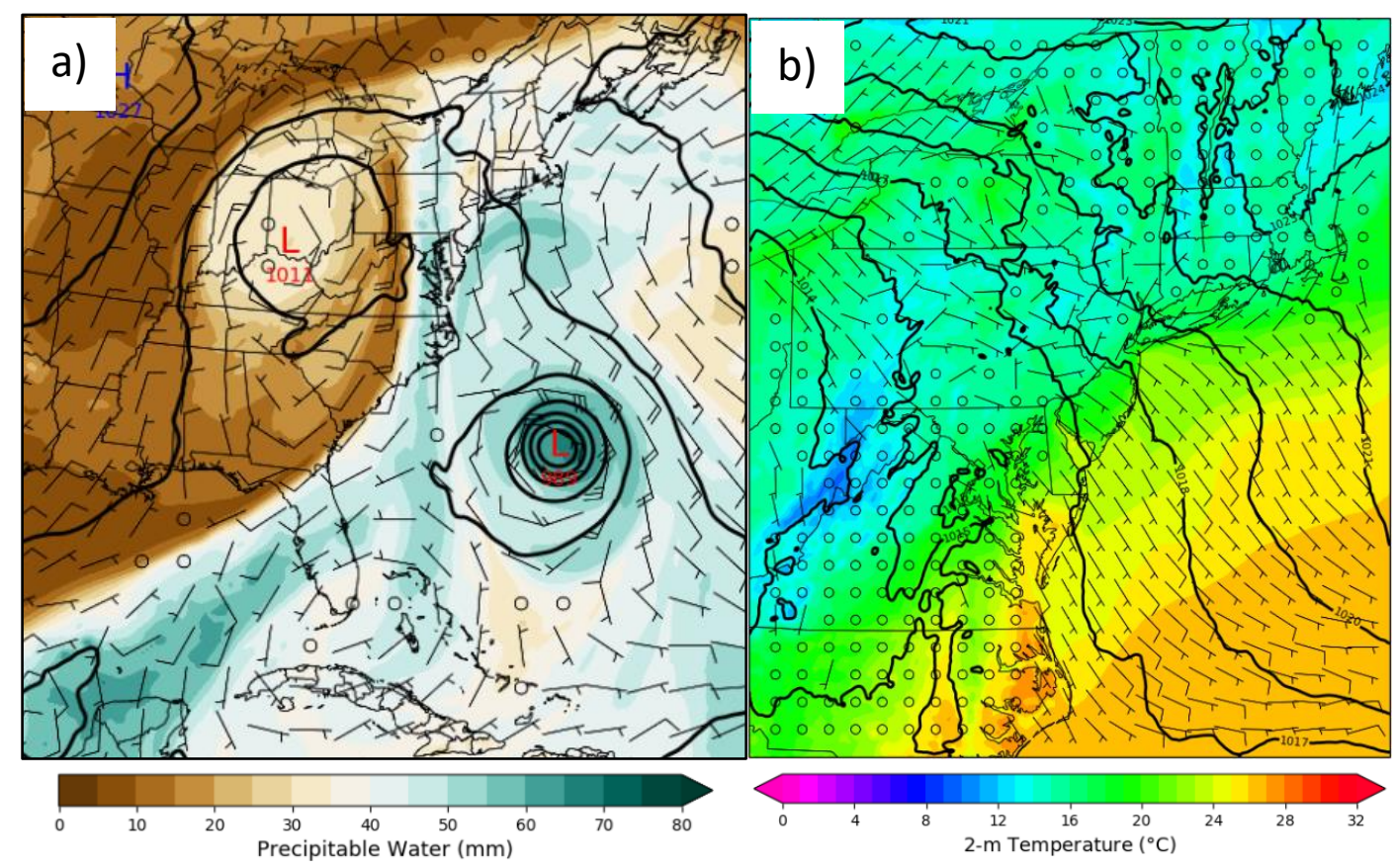

Figure 4.50. WRF results for the simulation with no surface heat fluxes at 0300 UTC 08 September 2011 displaying a) precipitable water ( $\mathrm{mm}$; shaded), sea level pressure ( $\mathrm{hPa}$; contoured), and 850-hPa wind (m $\mathrm{s}^{-1}$ ) from domain $1 \mathrm{and} \mathrm{b}$ ) sea level pressure ( $\mathrm{hPa}$; contoured), 10-m wind $\left(\mathrm{m} \mathrm{s}^{-1}\right)$, and 2-m temperature $\left({ }^{\circ} \mathrm{C}\right.$; shaded) from domain 2.

Removing latent heating allowed for a deeper extratropical cyclone, but a weaker upper-level jet (not shown) and a much weaker tropical cyclone (Fig. 4.49a). No surface temperature gradient was found across Pennsylvania and surface winds were easterly (Fig. 4.49b) instead of southeasterly (Fig. 4.38a), suggesting drier surface air across eastern Pennsylvania. The extratropical cyclone deepened and the tropical cyclone weakened when surface heat fluxes were removed (Fig. 4.50a). Precipitable water values across Pennsylvania decreased and much drier air was present along the East Coast in association with a deeper extratropical cyclone and increased winds at $850 \mathrm{hPa}$ (Fig. 4.50a). Surface temperatures were much cooler across Pennsylvania and surface winds 


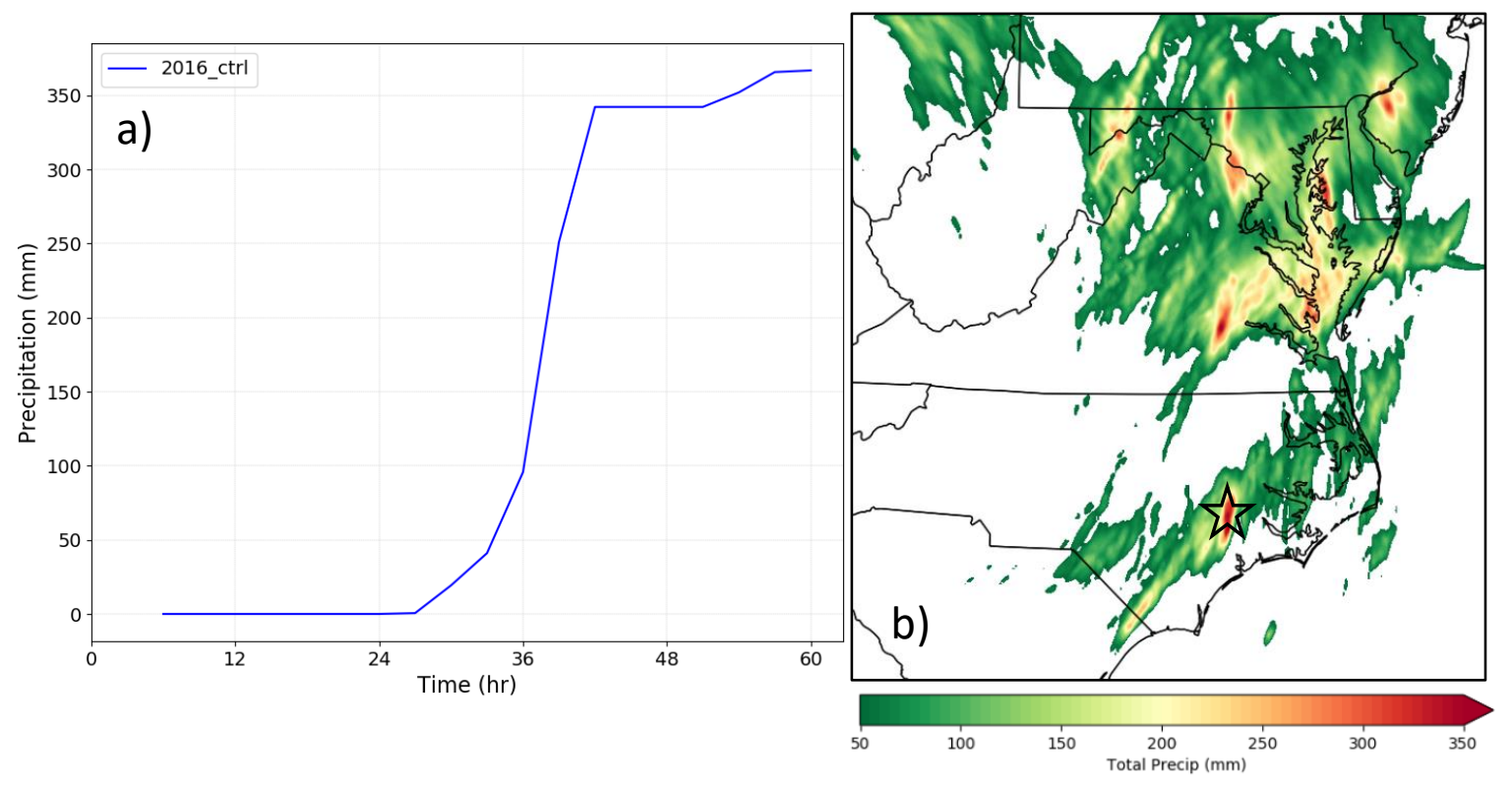

Figure 4.51. Precipitation (mm) for the 2016 event from 1200 UTC 28 September 2016 to 0000 UTC 01 October 2016 for domain 2 of the WRF control simulation a) at the location of maximum precipitation in North Carolina, plotted as accumulation with time and b) as the event total for the region. The transparent star represents the location of maximum precipitation.

were weaker from the east (Fig. 4.50b). If a thermal boundary played a role in the 2011 event, it was eliminated from the simulation when surface heat fluxes were removed, which could explain the $30 \%$ decrease in precipitation totals across Pennsylvania like that found in Colle (2003). Both surface heat fluxes and latent heating aided the convection for the 2011 flood, but latent heating had the most significant contribution.

\subsection{September 2016 - North Carolina}

Precipitation began around 1500 UTC 29 September 2016 for a location in eastern North Carolina in the WRF control simulation of the event, with the most intense rain 


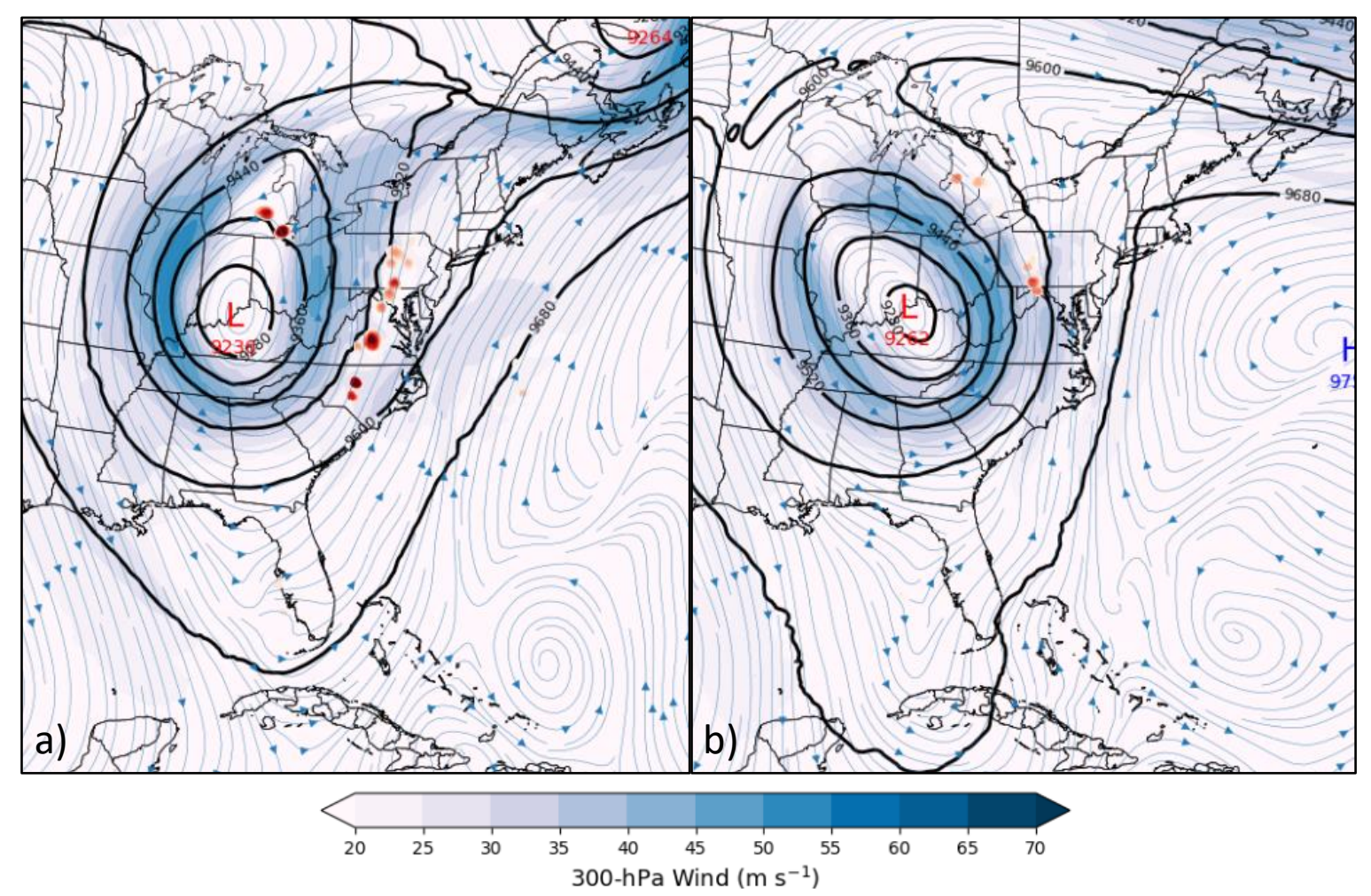

Figure 4.52. WRF domain $1300-\mathrm{hPa}$ height (gpm; thick contoured), wind ( $\mathrm{m} \mathrm{s}^{-1}$; shaded; thin streamlines), and divergence $\left(10^{-5} \mathrm{~s}^{-1}\right.$; contoured; positive red) at a) 1200 UTC 29 September 2016 and b) 1200 UTC 30 September 2016 of the control simulation.

falling from 0000 UTC to 0600 UTC 30 September 2016 (Fig. 4.51a). Nearly $250 \mathrm{~mm}$ of rain fell in just six hours (Fig. 4.51a), bringing the maximum total precipitation for the event to $366.83 \mathrm{~mm}$ (Fig. 4.51b). Several additional locations along the mid-Atlantic coast received upwards of $350 \mathrm{~mm}$ of rain, but the focus of this research was the location in North Carolina due to the high intensity of precipitation and the proximity to the actual event location of such rainfall (Fig. 2.15b). 


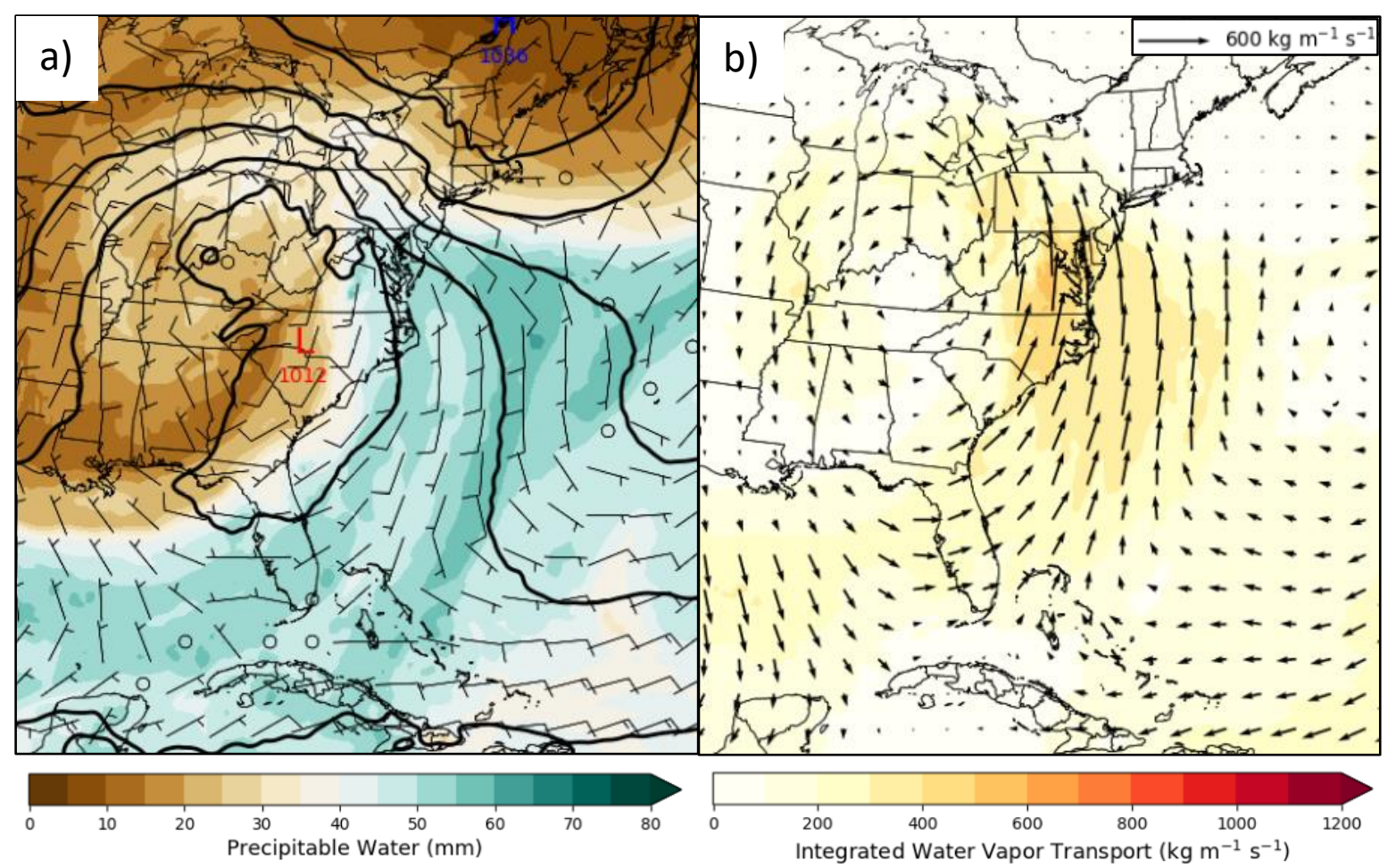

Figure 4.53. WRF results for domain 1 of the control simulation at 0000 UTC 30 September 2016 displaying a) precipitable water ( $\mathrm{mm}$; shaded), sea level pressure ( $\mathrm{hPa}$; contoured), 850-hPa wind ( $\mathrm{m} \mathrm{s}^{-1}$ ), and $\mathrm{b}$ ) integrated water vapor transport (IVT; $\mathrm{kg} \mathrm{m}^{-1} \mathrm{~s}^{-1}$ ).

\subsubsection{Synoptic Scale}

As with the 2015 and 2011 events, an amplified pattern in the upper levels resulted in a cut-off low over the eastern United States (Fig. 4.52a). A blocking ridge of high pressure over the Atlantic allowed for the upper-level low over the Ohio River Valley to become cut off from the trough over eastern Canada. In association with the low over the eastern United States, upper-level divergence and southerly flow were present along the mid-Atlantic coast at 1200 UTC 29 September 2016 (Fig. 4.52a), just prior to the onset of precipitation. The upper-level jet began to tilt westward as the area of 
low pressure remained over the Ohio River Valley through 1200 UTC 30 October 2016, placing North Carolina under southerly flow aloft (Fig. 4.52b).

The deep extratropical cyclone over the eastern United States allowed for southerly flow at all levels across North Carolina. As with the 2015 and 2011 events, the extratropical cyclone remained far enough west to allow precipitable water values to approach $50 \mathrm{~mm}$ for eastern North Carolina (Fig. 4.53a). The moisture flow in the 2016 event did not produce a narrow plume like the previous cases, but IVT was near $500 \mathrm{~kg}$ $\mathrm{m}^{-1} \mathrm{~s}^{-1}$ for the mid-Atlantic coast, with the moisture mainly coming from the region of the Gulf Stream (Fig. 4.53b). 


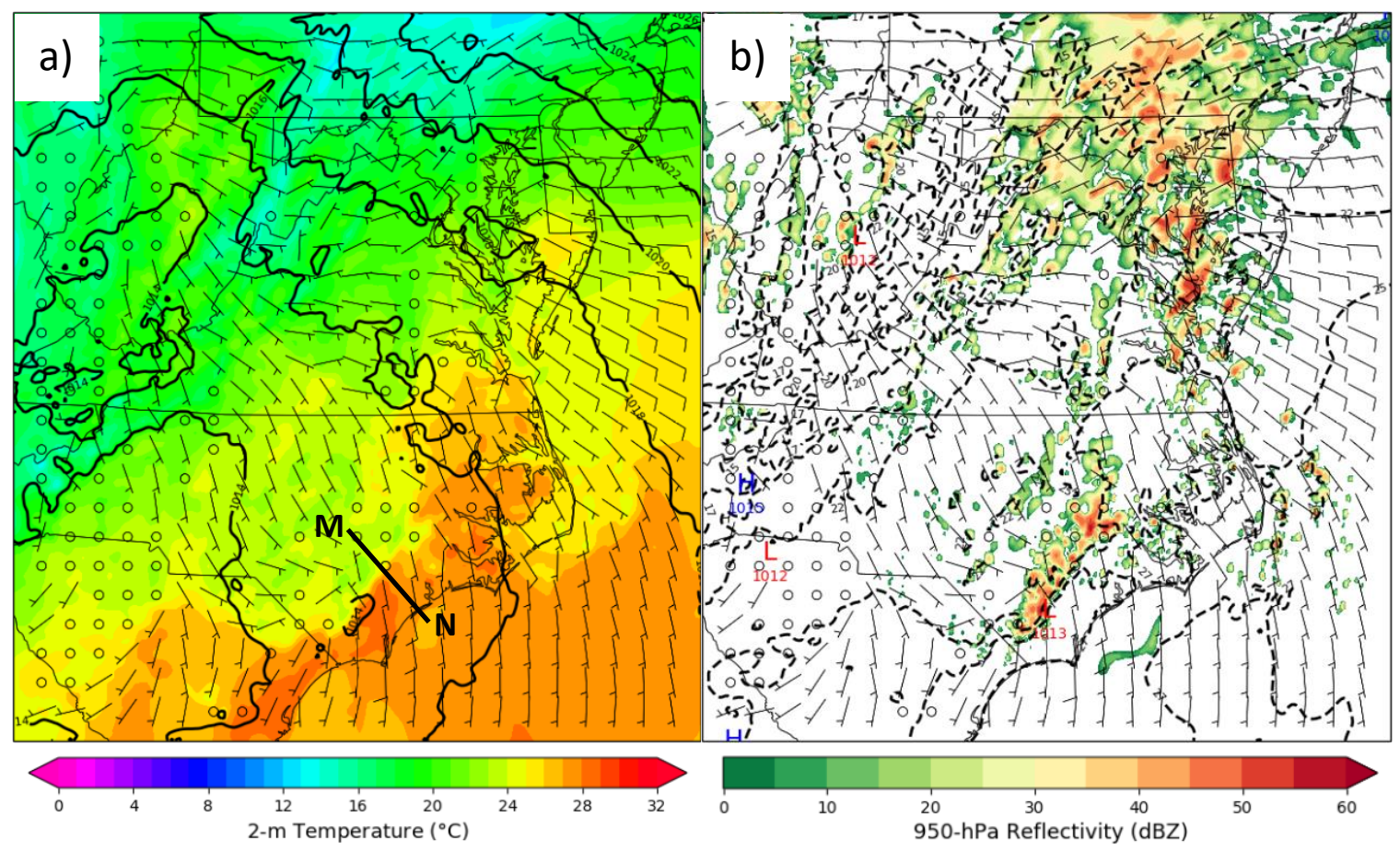

Figure 4.54. WRF results for domain 2 of the control simulation at 1500 UTC 29 September 2016 displaying $10-\mathrm{m}$ wind $\left(\mathrm{m} \mathrm{s}^{-1}\right)$, a) sea level pressure (hPa; contoured), 2-m temperature ( ${ }^{\circ} \mathrm{C}$; shaded), line segment $\mathrm{MN}, \mathrm{b}) 2-\mathrm{m}$ temperature $\left({ }^{\circ} \mathrm{C}\right.$; dashed), and 950-hPa reflectivity (dBZ; shaded).

\subsubsection{Mesoscale}

A temperature gradient and a clear wind shift was located along eastern North Carolina at 1500 UTC 29 September 2016 (Fig. 4.54a), when precipitation began (Fig. 4. 54a). Reflectivity values appeared along and just northwest of the temperature gradient (Fig. 4.54b), similar to the 2015 event, which suggested the possibility of a coastal front initiating convection in the region. Cross section $\mathrm{MN}$ provided evidence of a front with a thermally direct circulation near the area of precipitation ( $\sim 50-\mathrm{km}$ mark), as isentropes steeply sloped to the surface near the 70-km mark at 1800 UTC 29 September 2016 (Fig. 


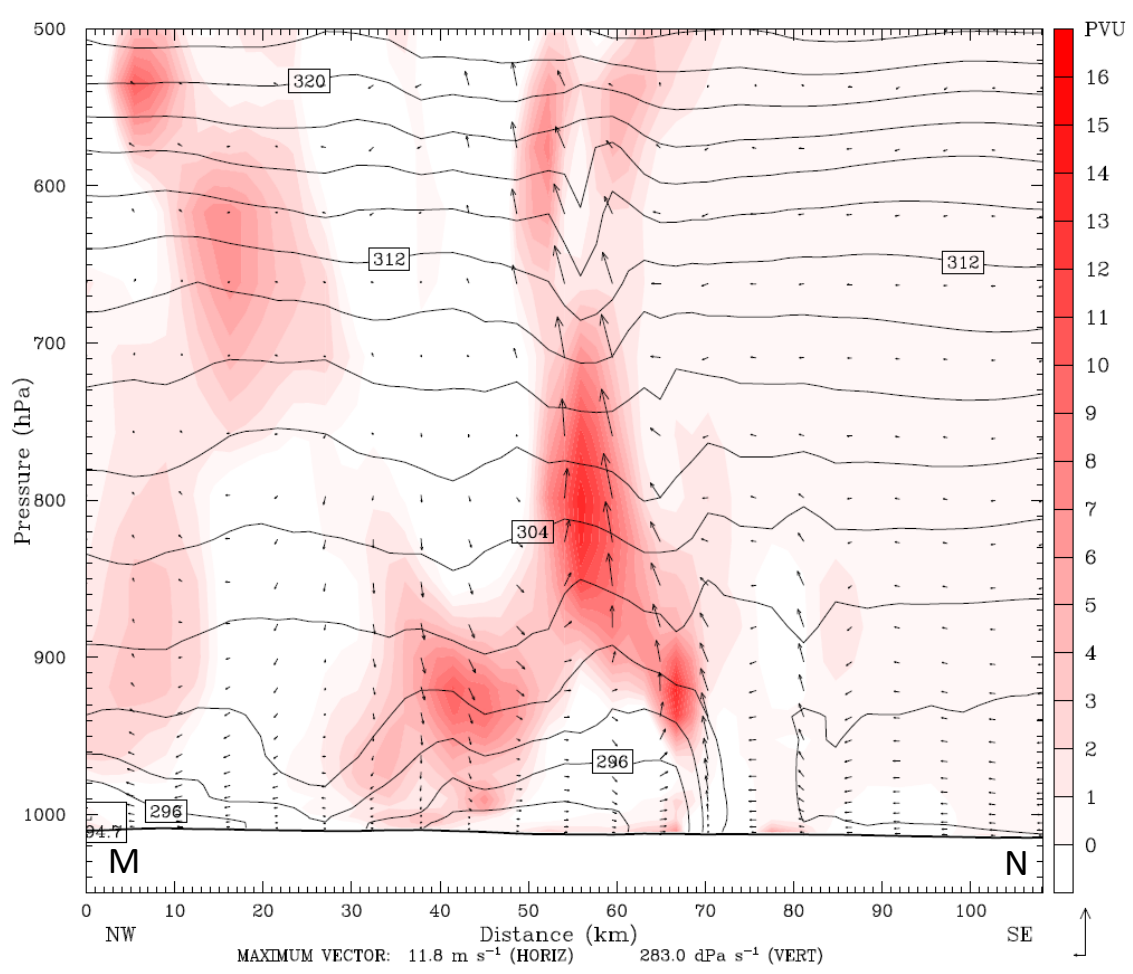

Figure 4.55. WRF domain 2 cross section from the control simulation at 1800 UTC 29 September 2016 for line segment MN (Fig. 4.54a) displaying potential vorticity (PVU; shaded red), potential temperature (K; contoured), and circulation vectors $\left[\mathrm{m} \mathrm{s}^{-1}, \mathrm{dPa} \mathrm{s}^{-1}\left(1 \mathrm{dPa} \mathrm{s}^{-1}=10^{-3} \mathrm{mb} \mathrm{s}^{-1}\right)\right]$.

4.55). Mid-level ascent with increased PV was located above the frontal circulation (Fig. $4.55)$, in the area of precipitation just before the increase in precipitation intensity.

The terms of Miller (1948) frontogenesis equation revealed the same pattern as the 2015 event. Tilting frontogenesis was negative in the mid-levels above the thermal boundary (Fig. 4.56a). Deformation (Fig. 4.56b) and divergence (Fig. 4.56c) frontogenesis were collocated and maximized in the low levels within the frontal boundary, increasing the thermally direct circulation below $850 \mathrm{hPa}$ and enhancing the band of precipitation within and on the cold side of the front. The positive diabatic frontogenesis in the mid-levels (Fig. 4.56d) was due to condensational warming as a 

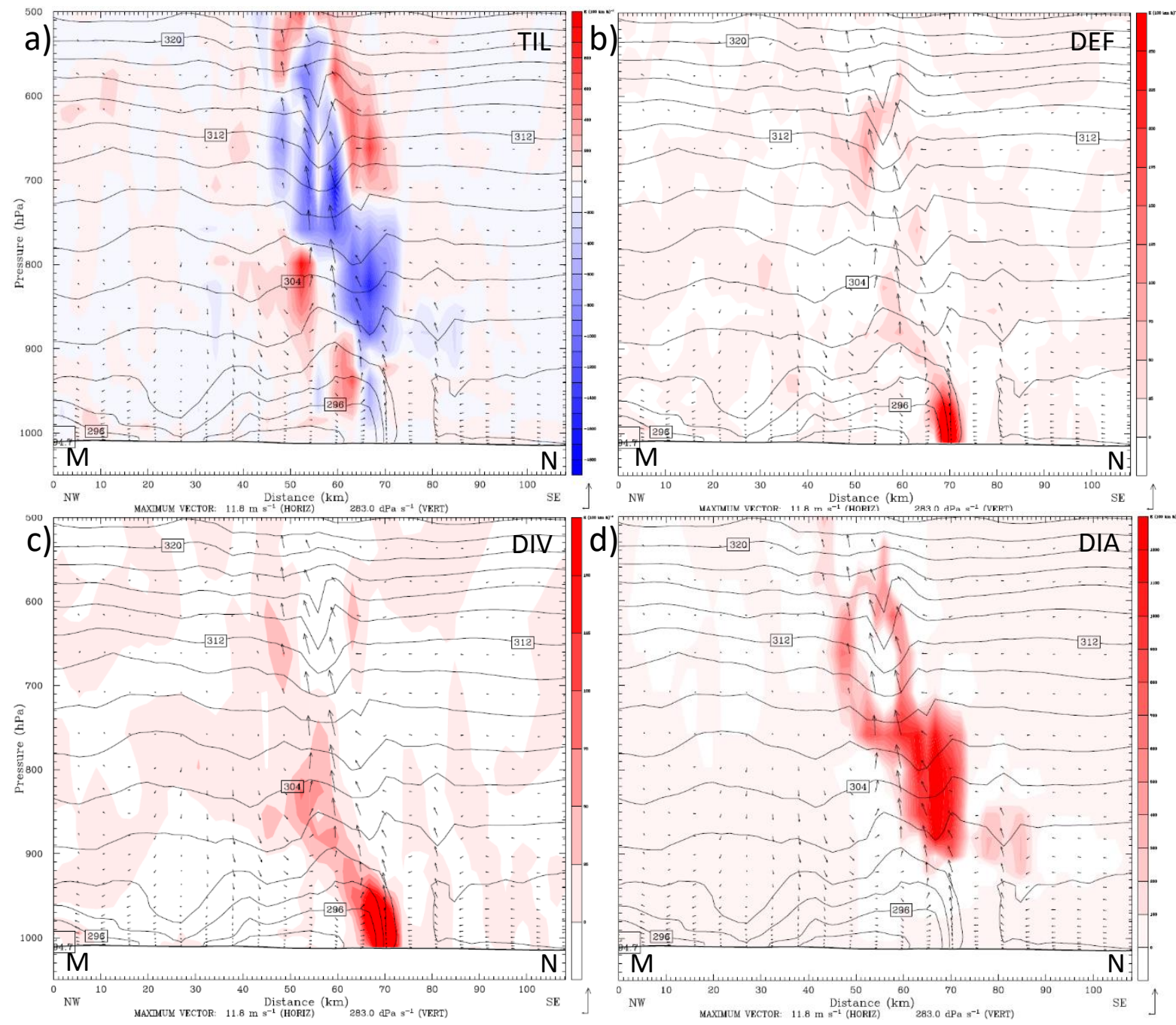

Figure 4.56. Cross sections MN (Fig. 4.54a) showing a) tilting, b) deformation, c) divergence, and d) diabatic frontogenesis [ $\mathrm{K}(100 \mathrm{~km} \mathrm{~h})^{-1}$; shaded; positive red, negative blue], potential temperature (K; contoured), and circulation vectors $\left[\mathrm{m} \mathrm{s}^{-1}, \mathrm{dPa} \mathrm{s}^{-1}\left(1 \mathrm{dPa} \mathrm{s}^{-1}=10^{-3} \mathrm{mb} \mathrm{s}^{-1}\right)\right]$ at 1800 UTC 29 September 2016 from WRF domain 2 of the control simulation.

result of the convection and led to the positive feedback system by enhancing the midlevel temperature gradient.

Within the frontal boundary, RH values were above 90\% (Fig. 4.57). The environment was saturated from the frontal boundary at the surface to $500 \mathrm{hPa}$ in a 


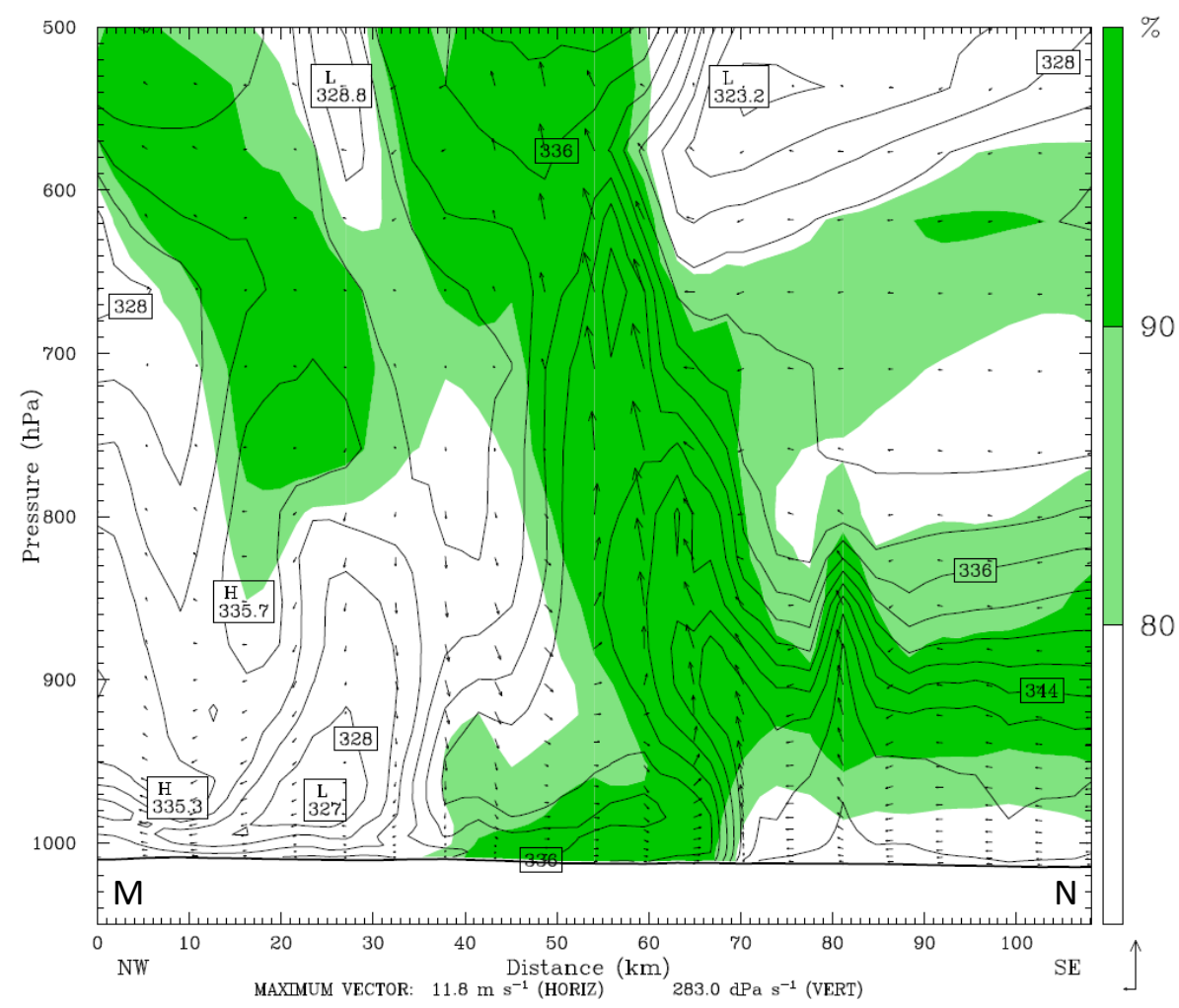

Figure 4.57. Cross sections MN (Fig. 4.54a) showing equivalent potential temperature ( $\mathrm{K}$; contoured), relative humidity (shaded), and circulation vectors $\left[\mathrm{m} \mathrm{s}^{-1}, \mathrm{dPa} \mathrm{s}^{-1}\left(1 \mathrm{dPa} \mathrm{s}^{-1}=10^{-3} \mathrm{mb} \mathrm{s}^{-1}\right)\right]$ at $1800 \mathrm{UTC} 29$ September 2016 from WRF domain 2 of the control simulation.

slantwise orientation towards the cold side of the boundary. The saturated region was consistent with a strong $\theta_{e}$ gradient, decreasing with height and steeply sloping to the surface (Fig. 4.57). The slantwise ascent occurred in the region of PI and increased RH (Fig. 4.57).

The cross sections suggested convection to be occurring in a saturated environment above a colder, stable layer near the surface. A sounding at the location of maximum precipitation at 1800 UTC 29 September 2016 showed the drier, stable layer from 1000 to $925 \mathrm{hPa}$, with the temperature profile nearly isothermal (Fig. 4.58). 


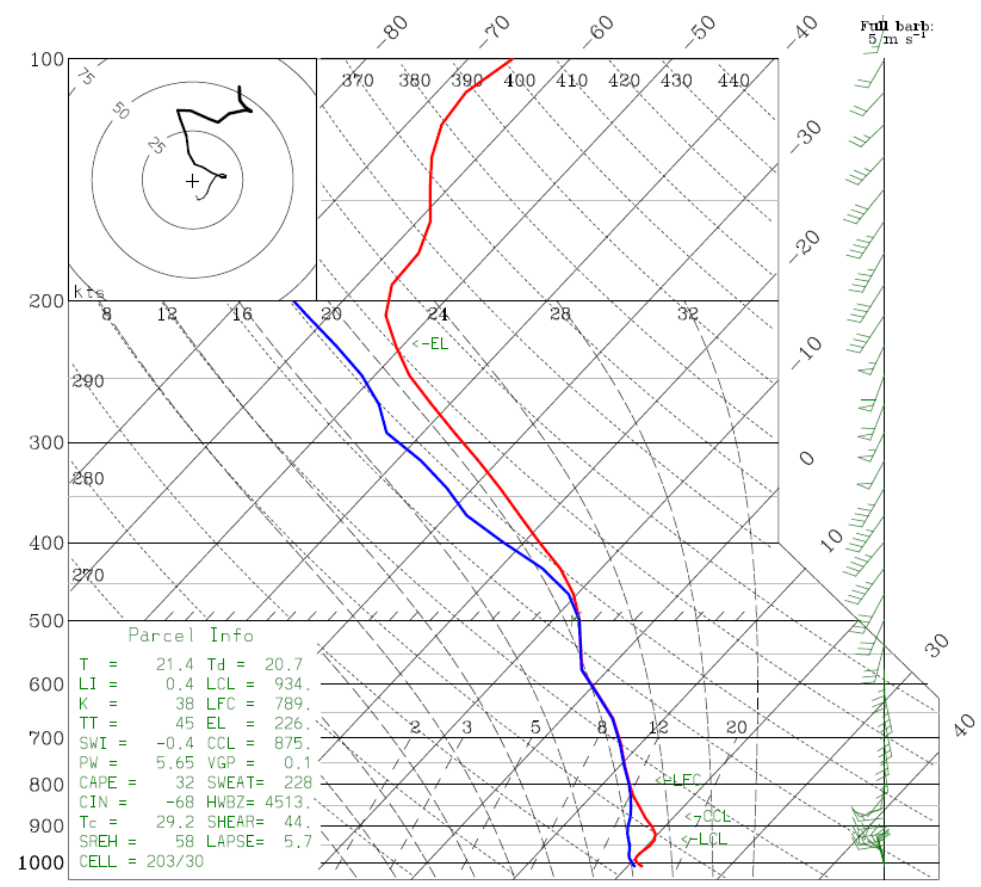

Figure 4.58. WRF domain 2 sounding from the control simulation at 1800 UTC 29 September 2016 for the location of maximum precipitation showing temperature $\left({ }^{\circ} \mathrm{C}\right.$; red), dew point $\left({ }^{\circ} \mathrm{C}\right.$; blue), and wind $\left(\mathrm{m} \mathrm{s}^{-1}\right)$.

The environment was saturated with strong southerly flow from 825 through $500 \mathrm{hPa}$ (Fig. 4.58). The saturated layer in the mid-levels was conditionally unstable, consistent with the cross-section analysis (Fig. 4.57), which could also be defined as a MAUL (Bryan and Fritsch 2000). With convection occurring above a stable, surface layer, the evidence pointed to elevated convection. However, the patterns were not as well defined as those of the 2015 event. 


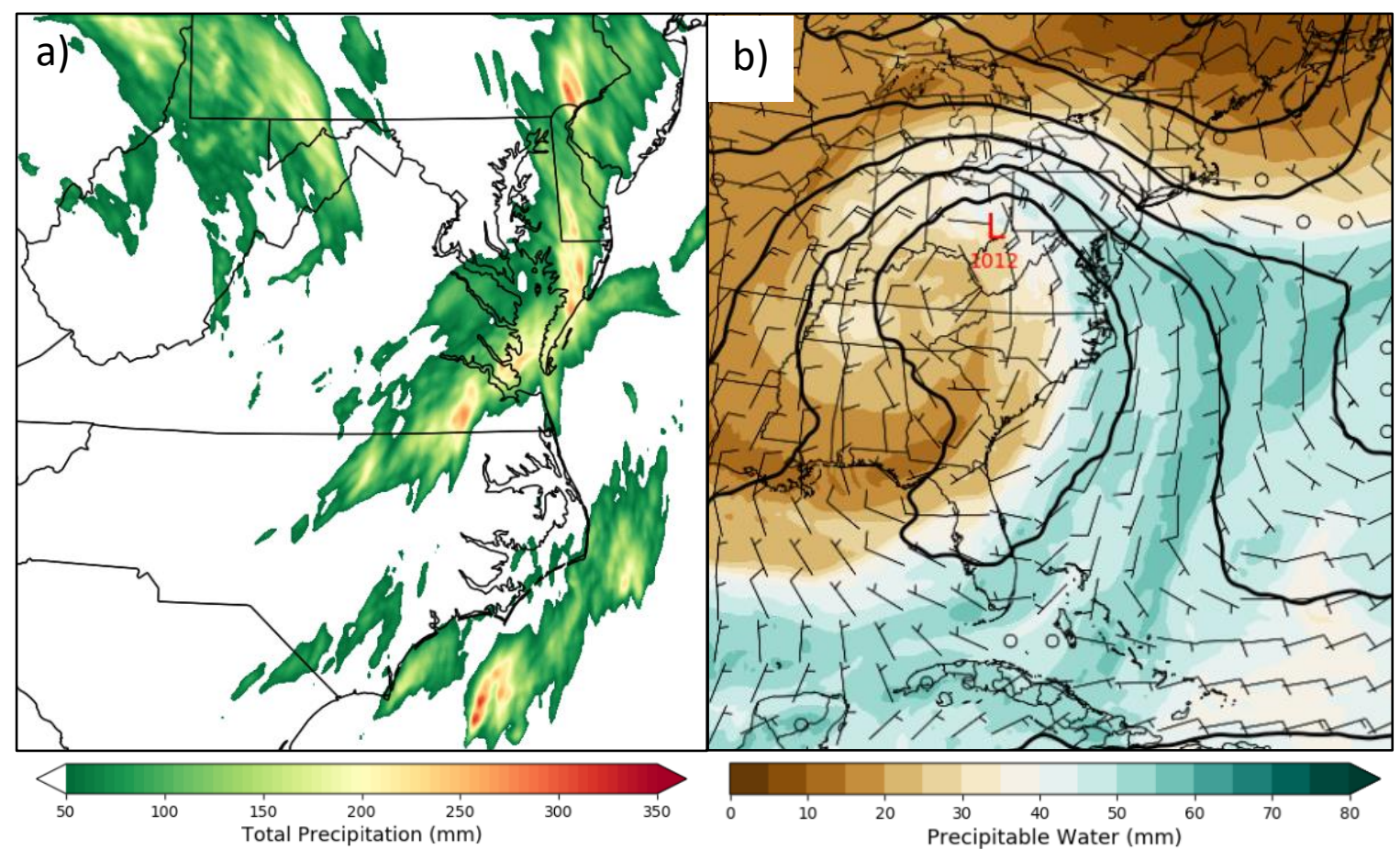

Figure 4.59. WRF results of the simulation with terrain removed displaying a) total accumulated precipitation $(\mathrm{mm})$ for domain 2 and b) precipitable water ( $\mathrm{mm}$; shaded), sea level pressure ( $\mathrm{hPa}$; contoured), and 850-hPa wind ( $\mathrm{m} \mathrm{s}^{-1}$ ) for domain 1 at 0000 UTC 30 September 2016.

\subsubsection{Terrain Influence}

The upper-level low deepened and shifted east by about $100 \mathrm{~km}$ when terrain was removed from the WRF simulation, but upper-level divergence remained over eastern North Carolina (not shown). The maximum precipitation in the domain remained above $300 \mathrm{~mm}$, while the location of that maximum shifted off the southern coast of North Carolina (Fig. 4.59a). The east shift of the extratropical cyclone resulted in a shift in the precipitable water pattern, decreasing the moisture available to eastern North Carolina (Fig. 4.59b). Additionally, precipitable water values were able to increase further inland across Pennsylvania and Ohio by 0000 UTC 30 September 2016 (Fig. 4.59b), which led 


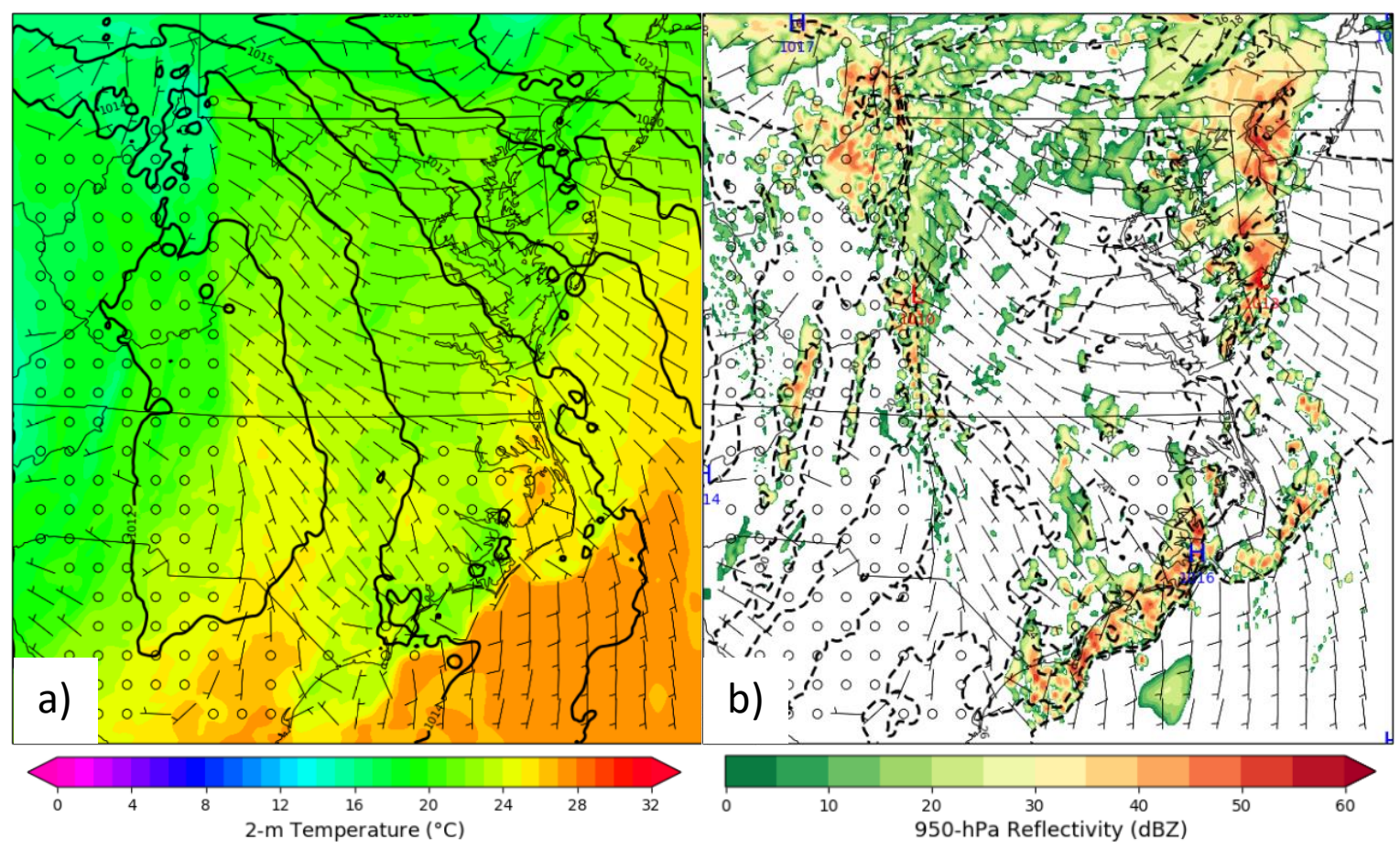

Figure 4.60. WRF results for domain 2 of the simulation with no terrain at 1500 UTC 29 September 2016 displaying 10-m wind $\left(\mathrm{m} \mathrm{s}^{-1}\right)$, a) sea level pressure ( $\mathrm{hPa}$; contoured), $2-\mathrm{m}$ temperature $\left({ }^{\circ} \mathrm{C}\right.$; shaded), b) $2-\mathrm{m}$ temperature $\left({ }^{\circ} \mathrm{C}\right.$; dashed), and $950-\mathrm{hPa}$ reflectivity (dBZ; shaded).

to increased precipitation in that region (Fig. 4.59a).

In response to the east shift in the environmental pattern, the surface temperature gradient and wind shift were also pushed east off the coast of North Carolina (Fig. 4.60a). The temperature gradient appeared slightly weaker than that of the control simulation, with temperatures on the southeast side of the front staying at or below $28^{\circ} \mathrm{C}$ (Fig. $4.60 \mathrm{a}$ ). The reflectivity field remained consistent with the control simulation; the precipitation fell at and northwest of the frontal boundary (Fig. 4.60b). Terrain seemed to have little influence on the initiation and maintenance of convection, but did impact the location of the intense precipitation. 


\subsubsection{Diabatic Processes}

Removing evaporation from precipitation in the WRF simulation led to similar rainfall over North Carolina (Fig. 4.61a) as that in the control simulation (Fig. 4.51b), except the field of totals above $300 \mathrm{~mm}$ was slightly smaller in area. A large difference was found in precipitation near Chesapeake Bay, with much higher totals along a northsouth oriented axis (Fig. 4.61a). Overall, the environmental pattern was nearly identical between the control simulation and the simulation without evaporation. The frontal boundary remained consistent, as seen with surface temperature and wind fields (not shown). Precipitation fell along and northwest of the boundary in North Carolina, as was the case with the control simulation.

Latent heating had the same influence on the 2016 event as it did on the 2011 event. No axes of precipitation were found along the coast of the mid-Atlantic states (Fig. 4.61b). The only precipitation that fell occurred along the Appalachian Mountains. There was no evidence of a frontal boundary, as persistent southeasterly surface winds were found across the state of North Carolina (not shown). Surface temperatures were warmer across North Carolina, with the eastern part of the state reaching $30^{\circ} \mathrm{C}$. The moisture pattern, along with the upper-level low and associated jet, remained consistent with that of the control simulation. However, upper-level winds increased from 20 to $30 \mathrm{~m} \mathrm{~s}^{-1}$ over east-central North Carolina (not shown).

Removing surface heat fluxes resulted in very little precipitation for North

Carolina, but had only minor impacts on the precipitation across Virginia and Maryland (Fig. 4.61c). The northern-most precipitation was not associated with the frontal 


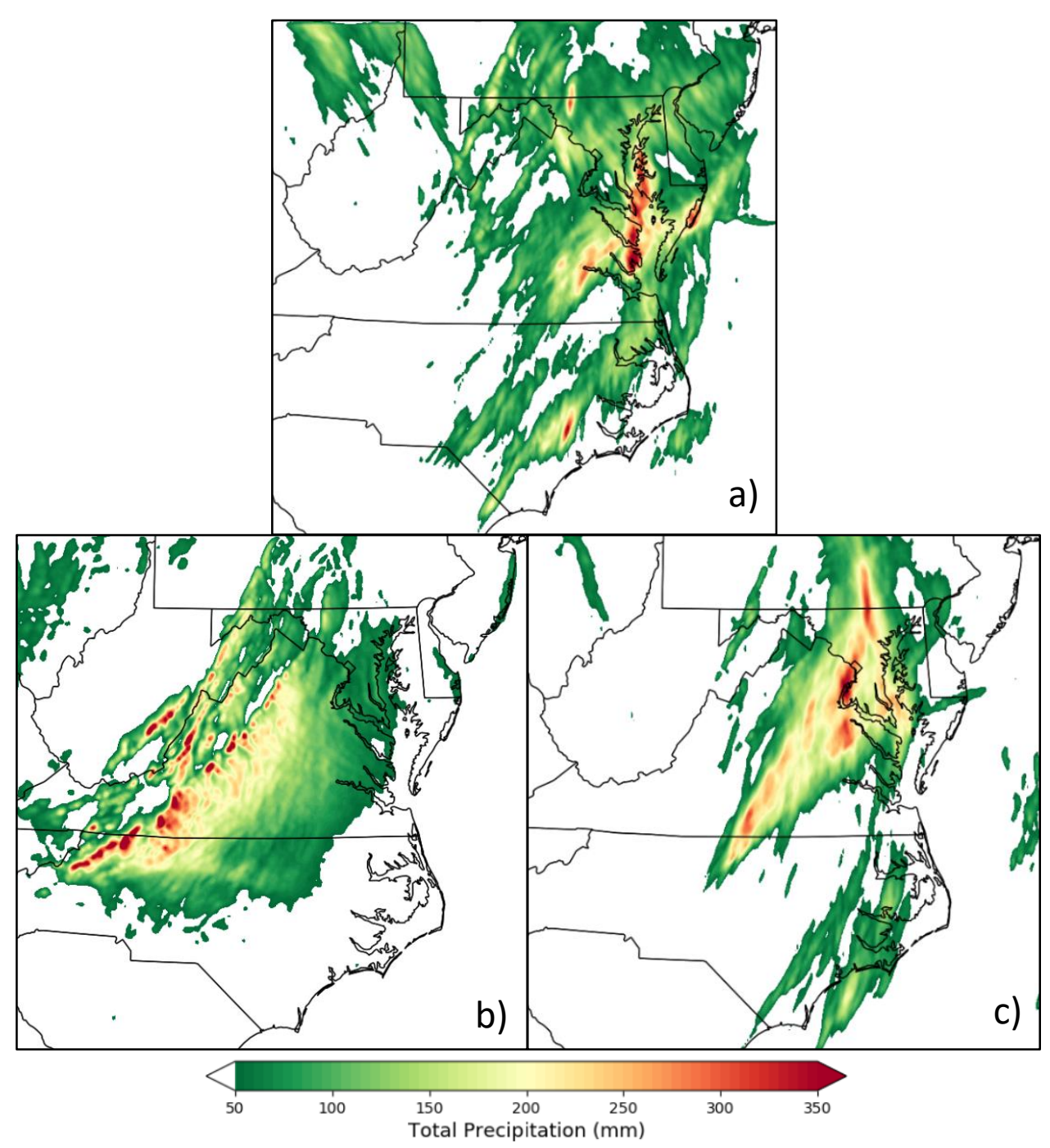

Figure 4.61. Total accumulated precipitation $(\mathrm{mm})$ from WRF domain 2 for the simulation with a) no evaporation from precipitation, b) no latent heating, and c) no surface heat fluxes.

boundary and was most impacted by condensational warming (Fig. 4.61b). The North Carolina region had lower precipitable water values, stronger 850-hPa wind from the south at $15 \mathrm{~m} \mathrm{~s}^{-1}$ at 0000 UTC 30 September 2016, and a deeper extratropical cyclone bringing drier air into western North Carolina when surface heat fluxes were removed (not shown). Additionally, there was no evidence of a coastal front across eastern North 


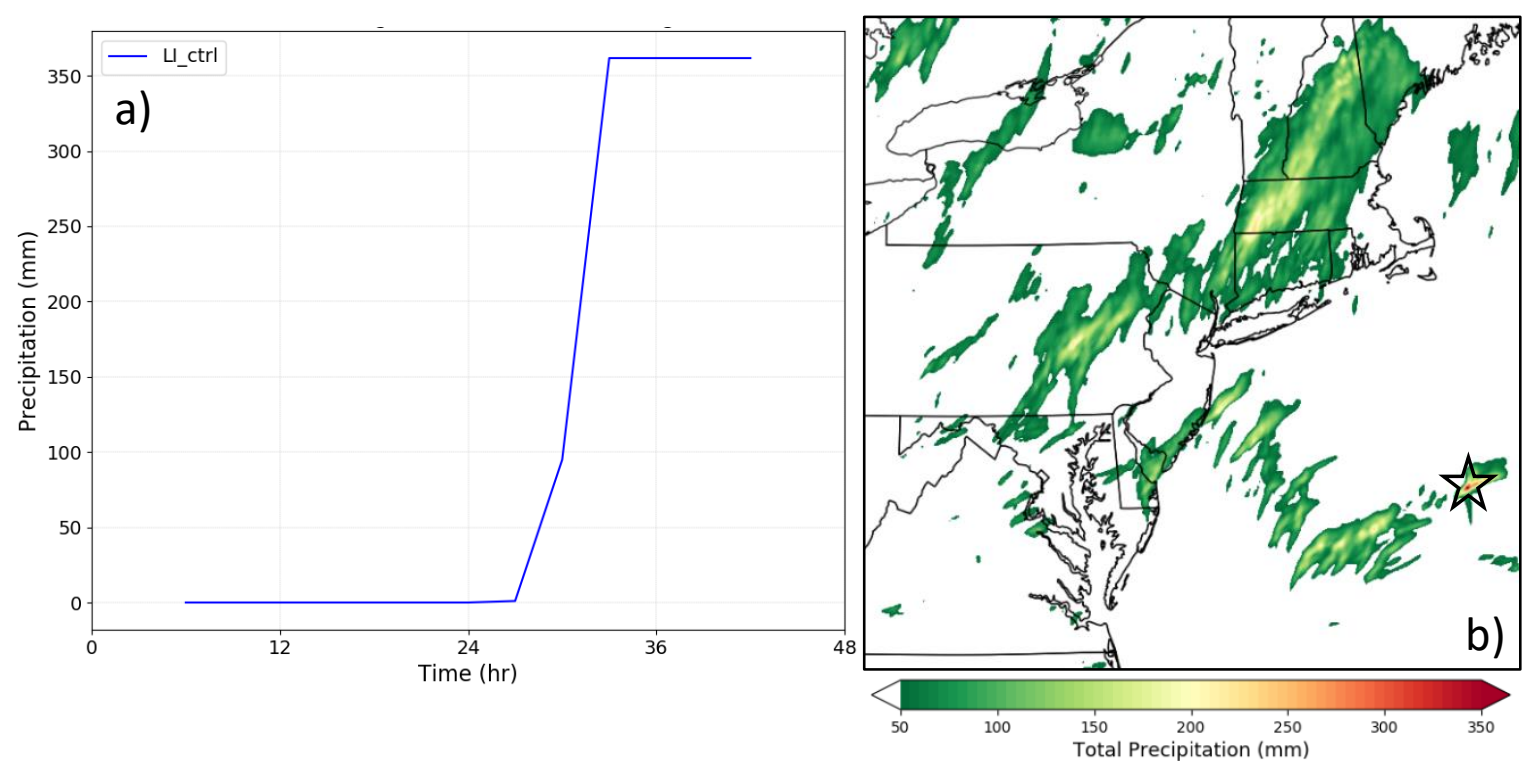

Figure 4.62. Precipitation (mm) for the 2014 event from 1200 UTC 12 August 2014 to 0600 UTC 14 August 2014 for domain 2 of the WRF control simulation a) at the location of maximum precipitation, displaced southeast of Long Island, New York, plotted as accumulation with time and b) as the event total for the region. The transparent star represents the location of maximum precipitation.

Carolina. With lower moisture availability and no front to provide lift, it was no surprise to see decreased precipitation totals.

\subsection{August 2014 - New York}

The control simulation of the intense precipitation in August 2014 resulted in a maximum of $361.74 \mathrm{~mm}$ of rain over a 6-hour period (Fig. 4.62a). The majority of that rain $(\sim 270 \mathrm{~mm})$ fell in only 3 hours from 1800 UTC to 2100 UTC 13 August 2014 (Fig. 4.62a). The location of the intense precipitation was well offshore (Fig. 4.62b), but the 


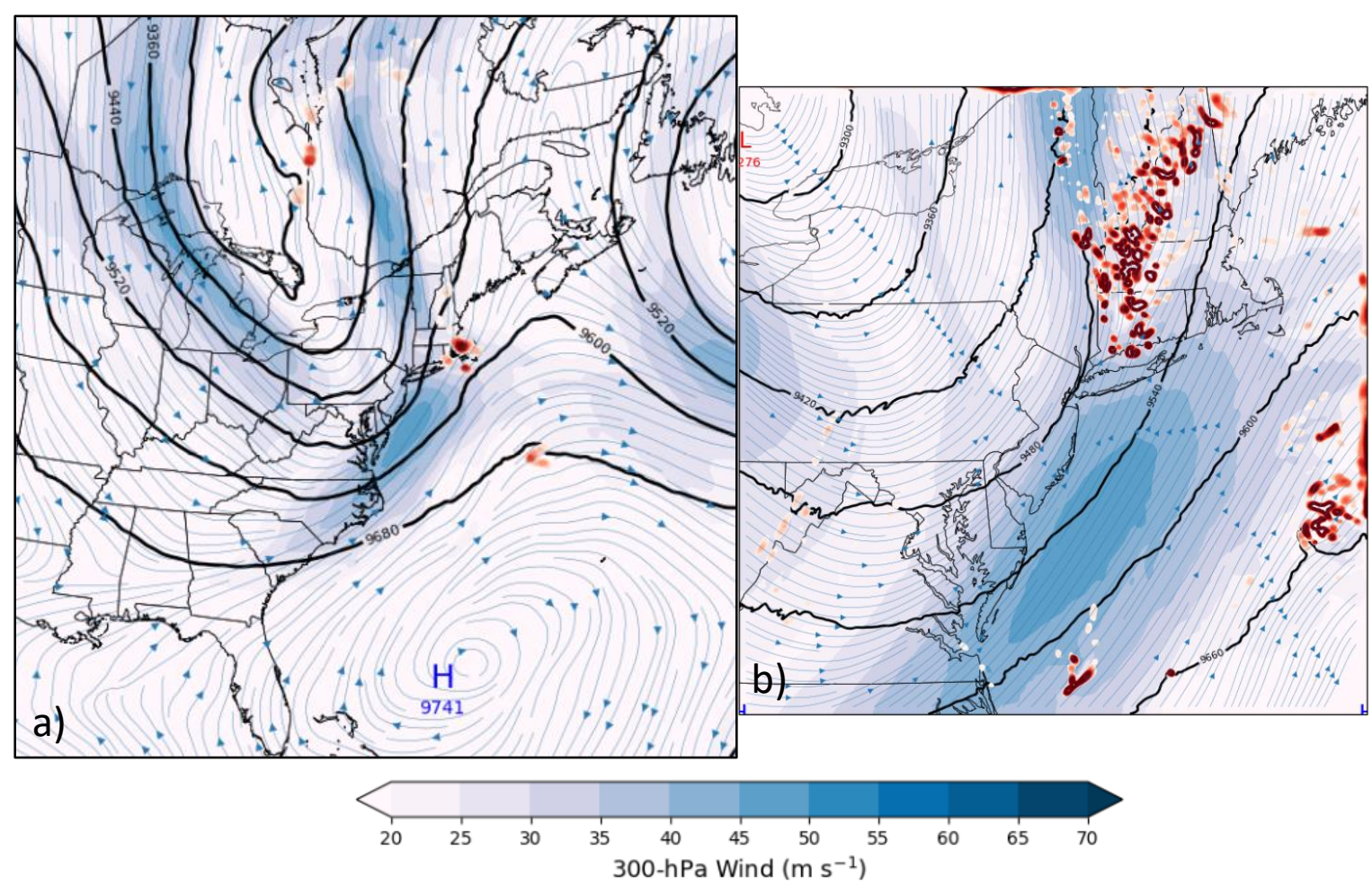

Figure 4.63. WRF control simulation results at 1800 UTC 13 August 2014 300-hPa height (gpm; thick contoured), wind ( $\mathrm{m} \mathrm{s}^{-1}$; shaded; thin streamlines), and divergence (10-5 $\mathrm{s}^{-1}$; contoured; positive red) for a) domain 1 and b) domain 2 of the control simulation.

orientation and area of the $300+\mathrm{mm}$ precipitation field were similar to that of the actual event in Long Island, New York (Fig. 2.17b).

\subsubsection{Synoptic Scale}

The 2014 event did not involve a stagnant cut-off low in the upper levels, as with the previous cases, but an upper-level trough was present across eastern Canada and the northeastern United States (Fig. 4.63a). An area of high pressure was located south of Bermuda and a jet streak associated with the negatively tilted trough became oriented 


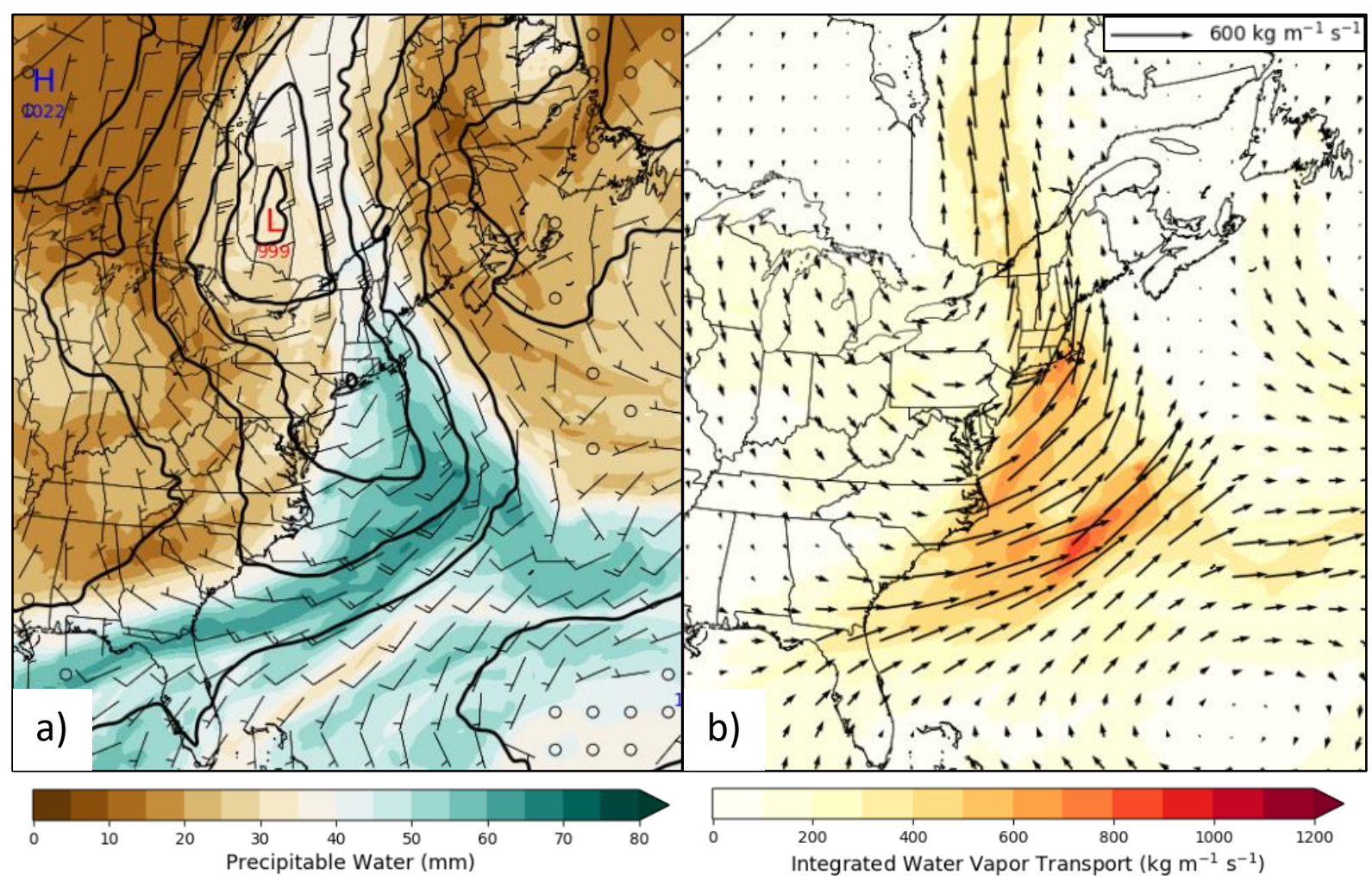

Figure 4.64. WRF results for domain 1 of the control simulation at 1800 UTC 13 August 2014 displaying a) precipitable water ( $\mathrm{mm}$; shaded), sea level pressure ( $\mathrm{hPa}$; contoured), 850-hPa wind ( $\mathrm{m} \mathrm{s}^{-1}$ ), and b) integrated water vapor transport (IVT; $\mathrm{kg} \mathrm{m}^{-1} \mathrm{~s}^{-1}$ ).

along the East Coast from Virginia to New York at 1800 UTC 13 August 2014 (Fig. 4.63a). Upper-level divergence was present over the region of maximum precipitation (Fig. 4.63b). Additionally, upper-level divergence was found over western Massachusetts and Connecticut in association with the left exit region of the jet streak off the coast and the right entrance region of an upper-level jet oriented north-south in eastern New York (Fig. 4.63b), consistent with a second area of precipitation totals above $250 \mathrm{~mm}$ (Fig. $4.62 b)$. 


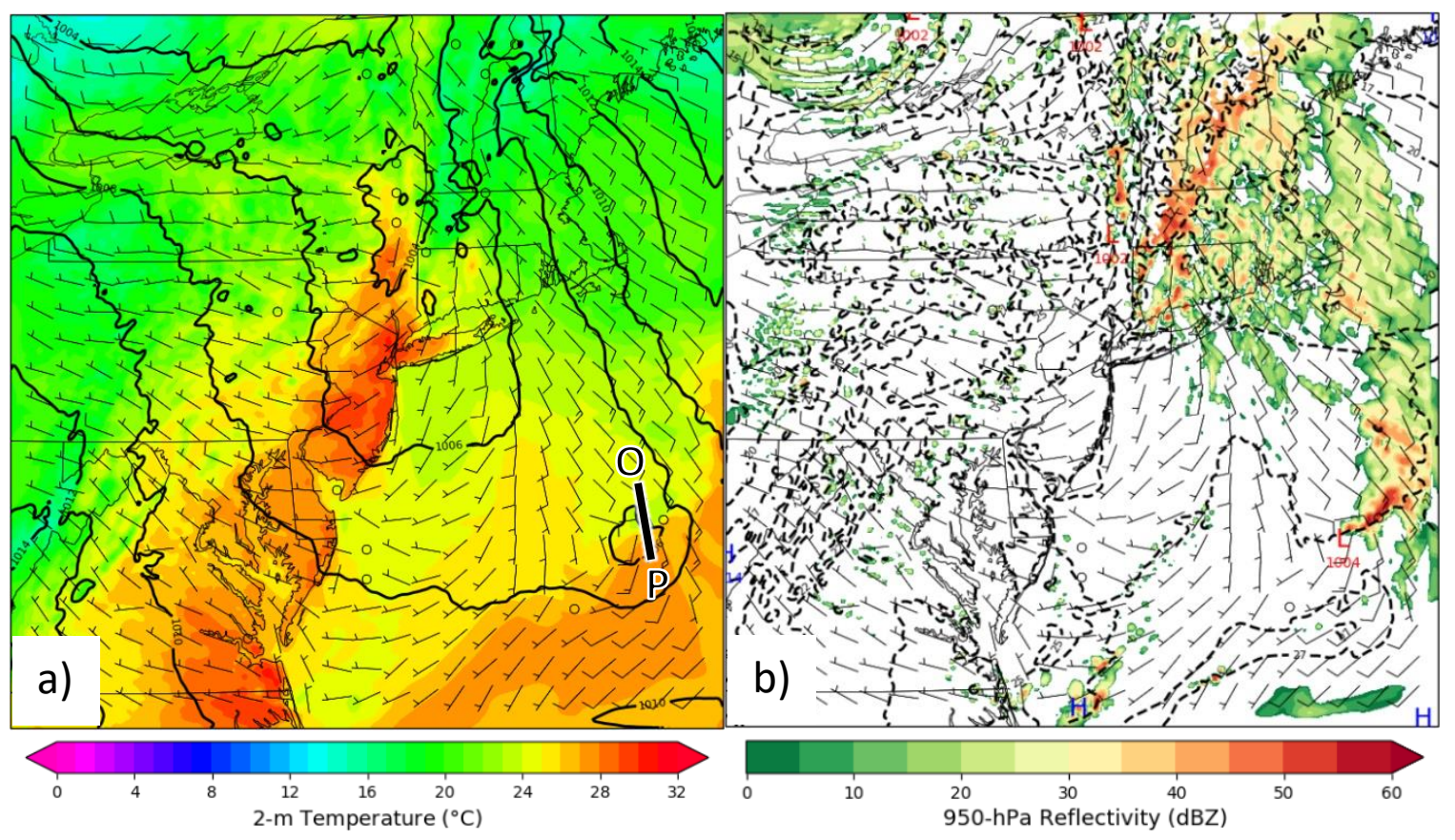

Figure 4.65. WRF results for domain 2 of the control simulation at 1800 UTC 13 August 2014 displaying 10$\mathrm{m}$ wind $\left(\mathrm{m} \mathrm{s}^{-1}\right)$, a) sea level pressure ( $\mathrm{hPa}$; contoured), 2-m temperature $\left({ }^{\circ} \mathrm{C}\right.$; shaded), line segment $\left.\mathrm{OP}, \mathrm{b}\right)$ 2-m temperature $\left({ }^{\circ} \mathrm{C}\right.$; dashed), and 950-hPa reflectivity (dBZ; shaded).

At $850 \mathrm{hPa}$, low pressure was located over eastern Canada at $1800 \mathrm{UTC} 13$ August 2014 (Fig. 4.64a) in association with the upper-level trough (Fig. 4.63a). Southwesterly flow at $850 \mathrm{hPa}$ off the coast of the mid-Atlantic states, combined with southeasterly flow off the coast of New England (Fig. 4.64a), allowed for higher precipitable water values over both locations of heavy precipitation. IVT values approached $600 \mathrm{~kg} \mathrm{~m}^{-1} \mathrm{~s}^{-1}$ southeast of Long Island (Fig. 4.64b), due to the flow associated with the aforementioned area of low pressure. As with the previous cases, the synoptic pattern contributed to the moisture and lift needed to produce the intense precipitation. 


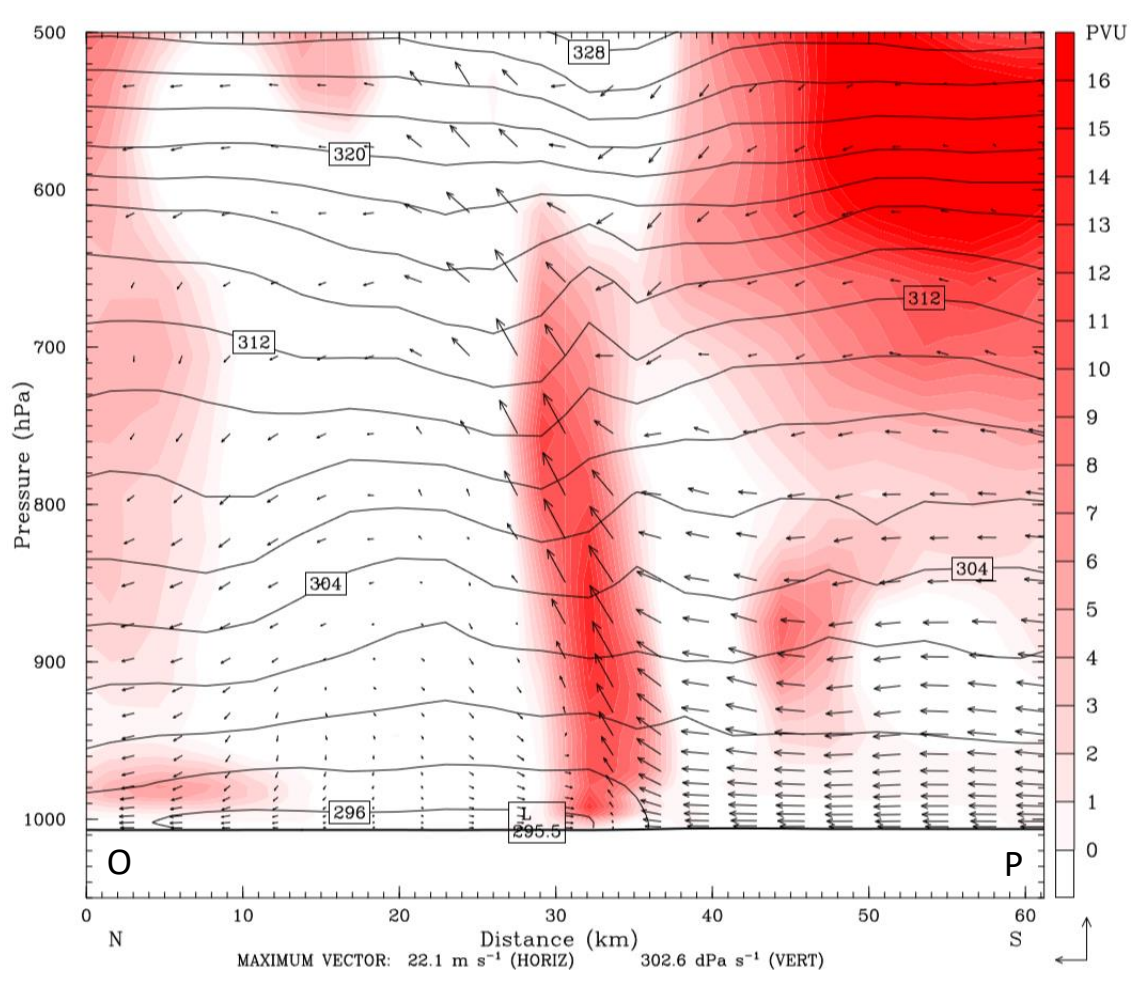

Figure 4.66. WRF domain 2 cross section from the control simulation at 1800 UTC 13 August 2014 for line segment OP (Fig. 4.65a) displaying potential vorticity (PVU; shaded red), potential temperature ( $\mathrm{K}$; contoured), and circulation vectors $\left[\mathrm{m} \mathrm{s}^{-1}, \mathrm{dPa} \mathrm{s}^{-1}\left(1 \mathrm{dPa} \mathrm{s}^{-1}=10^{-3} \mathrm{mb} \mathrm{s}^{-1}\right)\right]$.

\subsubsection{Mesoscale}

The surface wind field was representative of the upper levels, with west-

northwesterly flow over the northeastern states and south-southeasterly flow offshore of New England (Fig. 4.65a). Two areas of low pressure appeared at the surface at 1800 UTC 13 August 2014, just southwest of the regions of heavy precipitation (Fig. 4.65b). From the surface temperature and surface wind fields, it was difficult to determine the location of any frontal boundaries. 

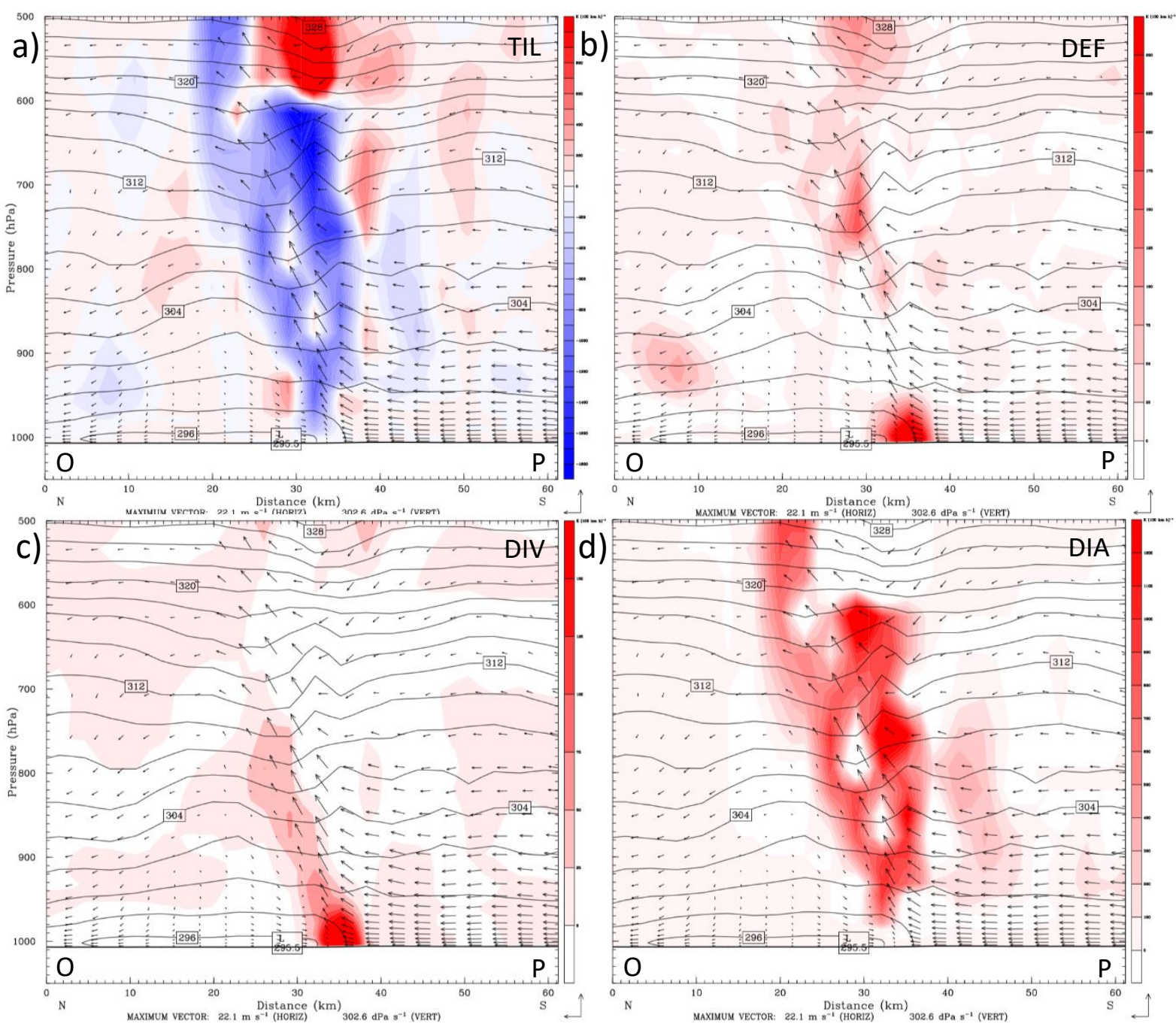

Figure 4.67. Cross sections OP (Fig. 4.65a) showing a) tilting, b) deformation, c) divergence, and d) diabatic frontogenesis [ $\mathrm{K}\left(100 \mathrm{~km} \mathrm{~h}^{-1}\right.$; shaded; positive red, negative blue], potential temperature ( $\mathrm{K}$; contoured), and circulation vectors [ $\left.\mathrm{m} \mathrm{s}^{-1}, \mathrm{dPa} \mathrm{s}^{-1}\left(1 \mathrm{dPa} \mathrm{s}^{-1}=10^{-3} \mathrm{mb} \mathrm{s}^{-1}\right)\right]$ at 1800 UTC 13 August 2014 from WRF domain 2 of the control simulation.

Focusing on the area of maximum precipitation southeast of Long Island, New York, a cross section perpendicular to the intense rainfall (Fig. 4.65a, b) showed evidence of a thermal boundary (Fig. 4.66) on the eastern edge of the surface low pressure center. A weak gradient in potential temperature was evident with only the $296 \mathrm{~K}$ and $298 \mathrm{~K}$ 


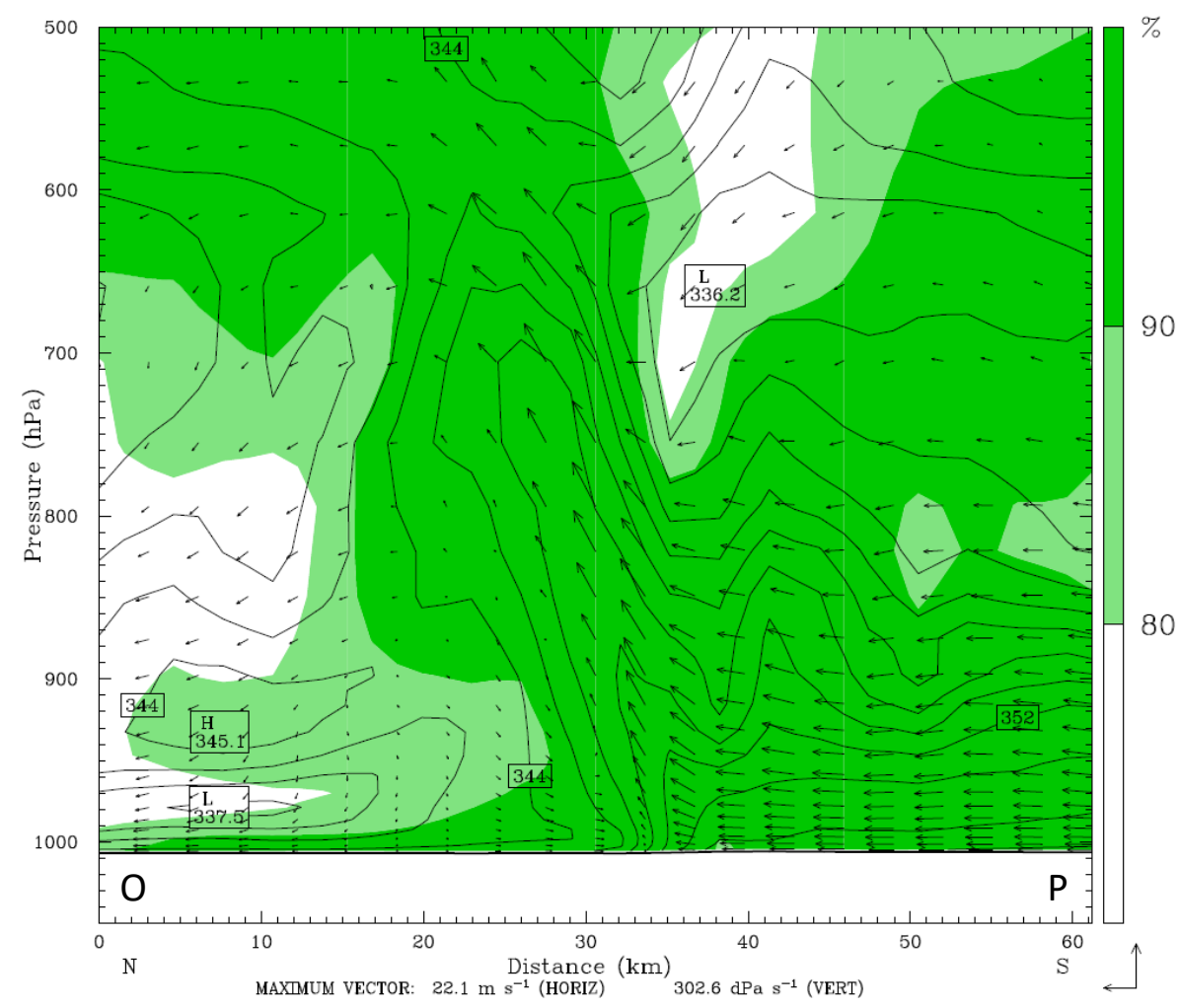

Figure 4.68. Cross sections OP (Fig. 4.65a) showing equivalent potential temperature (K; contoured), a) relative humidity (shaded), and circulation vectors $\left[\mathrm{m} \mathrm{s}^{-1}, \mathrm{dPa} \mathrm{s}^{-1}\left(1 \mathrm{dPa} \mathrm{s}^{-1}=10^{-3} \mathrm{mb} \mathrm{s}^{-1}\right)\right]$ at 1800 UTC 13 August 2014 from WRF domain 2 of the control simulation.

isentropic surfaces steeply sloping downward below $950 \mathrm{hPa}$ (Fig. 4.66). Additionally, strong southerly winds approached the thermal boundary, strong ascent from 950 to 700 $\mathrm{hPa}$ was present above the boundary in association with a region of $\mathrm{PV}$, and a weak northerly component to the wind was found on the cold side of the boundary (Fig. 4.66).

Frontogenesis patterns were consistent with the 2015 and 2016 events at the time of intense precipitation. Negative tilting frontogenesis was found in the region of ascent above the thermal boundary (Fig. 4.67a), deformation (Fig. 4.67b) and divergence (Fig. 4.67c) were contributing to frontogenesis and the thermally direct circulation near the 


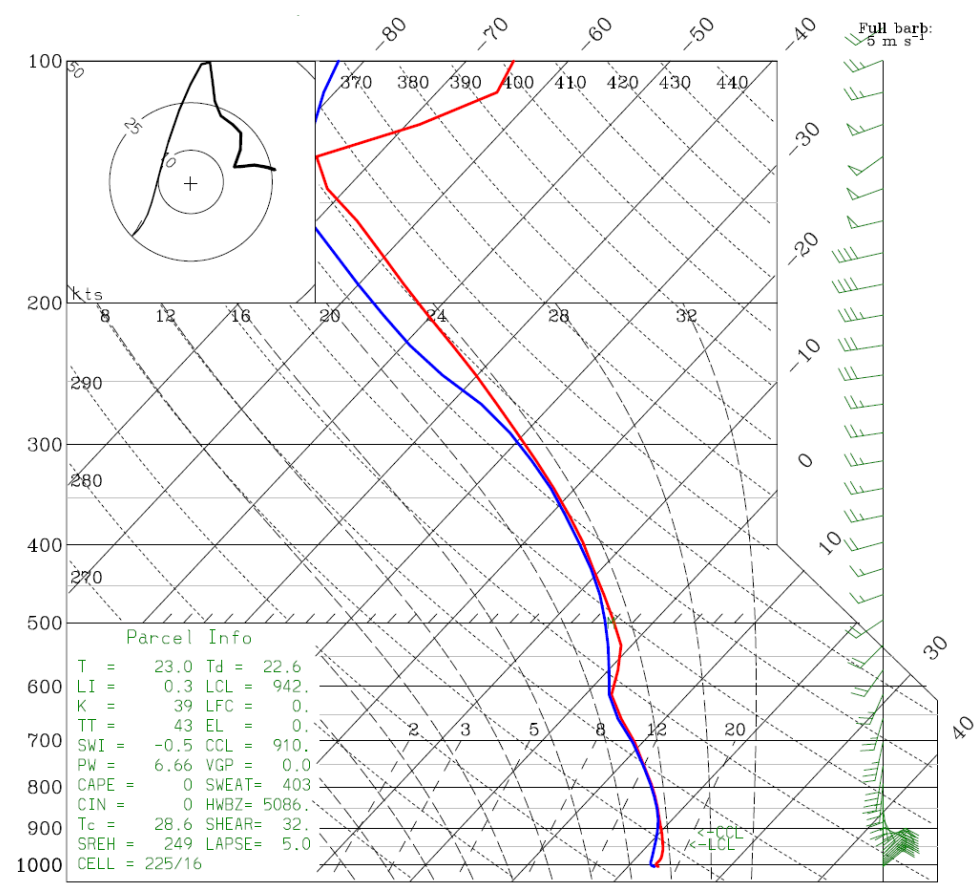

Figure 4.69. WRF sounding from domain 2 of the control simulation at 1800 UTC 13 August 2014 for the location of maximum precipitation showing temperature $\left({ }^{\circ} \mathrm{C}\right.$; red), dew point $\left({ }^{\circ} \mathrm{C}\right.$; blue), and wind $\left(\mathrm{m} \mathrm{s}^{-1}\right)$.

surface at the boundary, while strong diabatic frontogenesis was found in the mid-levels above the front (Fig. 4.67d) at 1800 UTC 13 August 2014. A region of deformation frontogenesis was also found in the mid-levels above the boundary (Fig. 4.67b) and divergence frontogenesis stretched from the surface to $750 \mathrm{hPa}$ above the front (Fig. $4.67 \mathrm{c})$.

Equivalent potential temperature decreased with height in the region of strong ascent from the surface to $600 \mathrm{hPa}$, as well as in the low levels south of the thermal boundary (Fig. 4.68). The same areas had RH values above 90\%. The region of PI and strong ascent within and above the frontal boundary suggested the release of gravitational instability, as with the 2015 and 2016 events. 
The thermal gradient was not strong enough to show an inversion near the surface at the location of maximum rainfall at 1800 UTC 13 August 2014 (Fig. 4.69). However, the environment was not saturated below $900 \mathrm{hPa}$, making it conditionally stable between 950 and $900 \mathrm{hPa}$ and absolutely stable between 1000 and $950 \mathrm{hPa}$, with veering winds between 950 and $750 \mathrm{hPa}$. From 900 to $620 \mathrm{hPa}$, the column was saturated and conditionally unstable (Fig. 4.69), consistent with the results of the cross section between the 20- and 25-km distances (Fig. 4.68).

\subsubsection{Terrain Influence}

Removing terrain limited precipitation over land for the 2014 event, but still resulted in more than $250 \mathrm{~mm}$ of rain offshore (Fig. 4.70a). The reduced rainfall amounts and offset location were likely due to a change in surface pressure when terrain was removed. A surface low was not present in the sea level pressure pattern southeast of Long Island nor over eastern New York (Fig. 4.70b). Surface temperatures were slightly cooler along the East Coast when terrain was removed (Fig. 4.70b), as compared to the control simulation (Fig. 4.65a), and frontal boundaries were still difficult to identify.

There was no difference in precipitable water, IVT, or upper-level dynamics between the WRF simulation with terrain and that without (not shown), except an upper-level shortwave trough was only present southeast of Long Island in accordance with the surface low the control simulation (Fig. 4.63b). The decreased precipitation seemed to be related to the lack of cyclonic circulations near the surface (Fig. 4.70b), which likely resulted in less surface convergence and lift. 


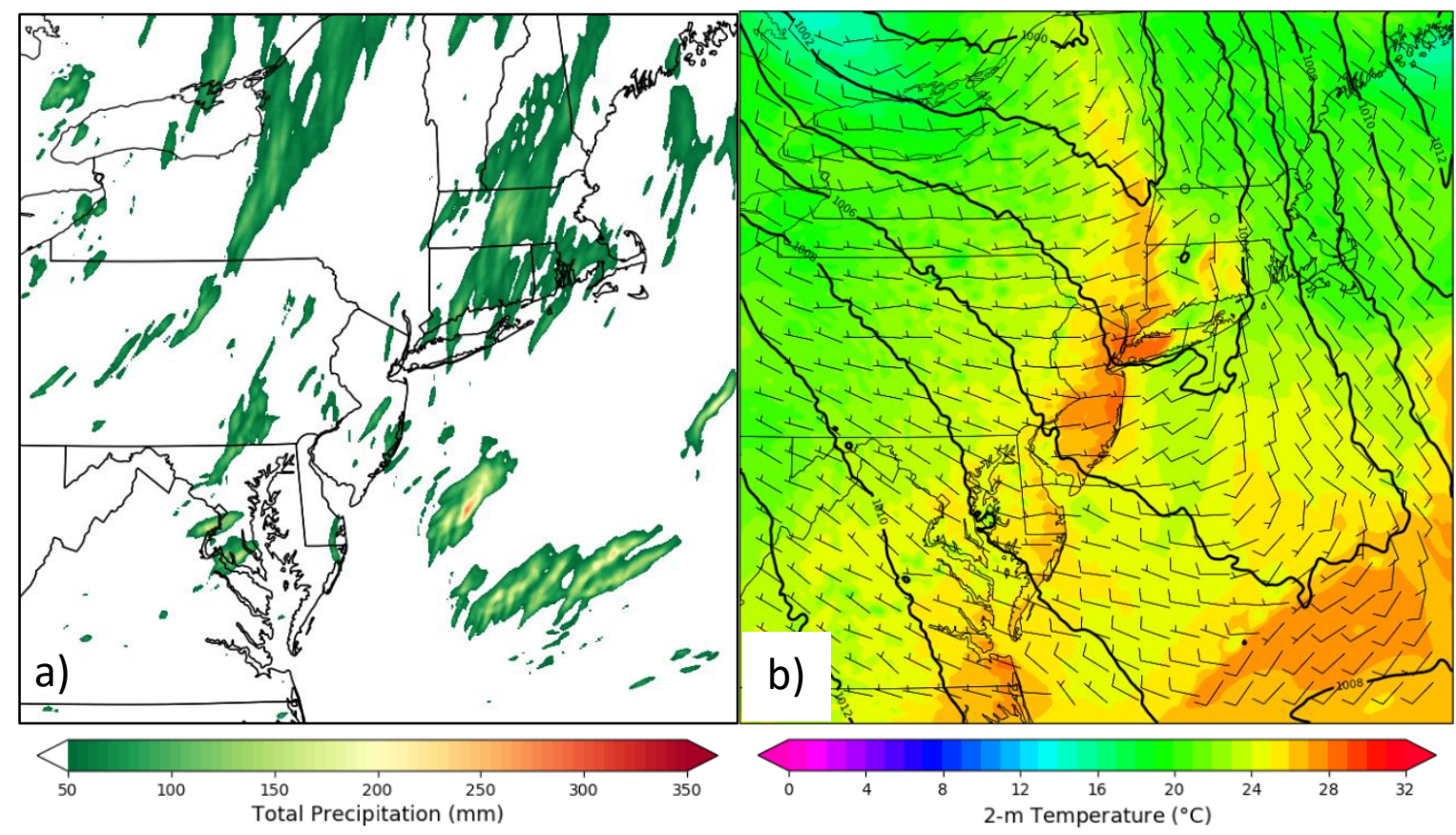

Figure 4.70. WRF results from domain 2 of the simulation with terrain removed displaying a) total accumulated precipitation $(\mathrm{mm})$ and $\mathrm{b})$ sea level pressure $\left(\mathrm{hPa}\right.$; contoured), 2-m temperature $\left({ }^{\circ} \mathrm{C}\right.$; shaded), and 10-m wind ( $\left.\mathrm{m} \mathrm{s}^{-1}\right)$ at 1800 UTC 13 August 2014.

\subsubsection{Diabatic Processes}

Evaporation from precipitation had a minor impact on the cyclonic circulation near the surface southeast of Long Island, New York (not shown). The overall precipitation amount was similar for much of the WRF domain when evaporation was removed (Fig. 4.71a), except in the region of maximum precipitation examined in the control simulation. Total precipitation decreased in the location of interest southeast of Long Island by nearly $300 \mathrm{~mm}$ when evaporation from precipitation was no longer able to contribute to the convective processes (Fig. 4.71a), which suggested the intense precipitation was mainly attributed to a positive feedback system once convection 


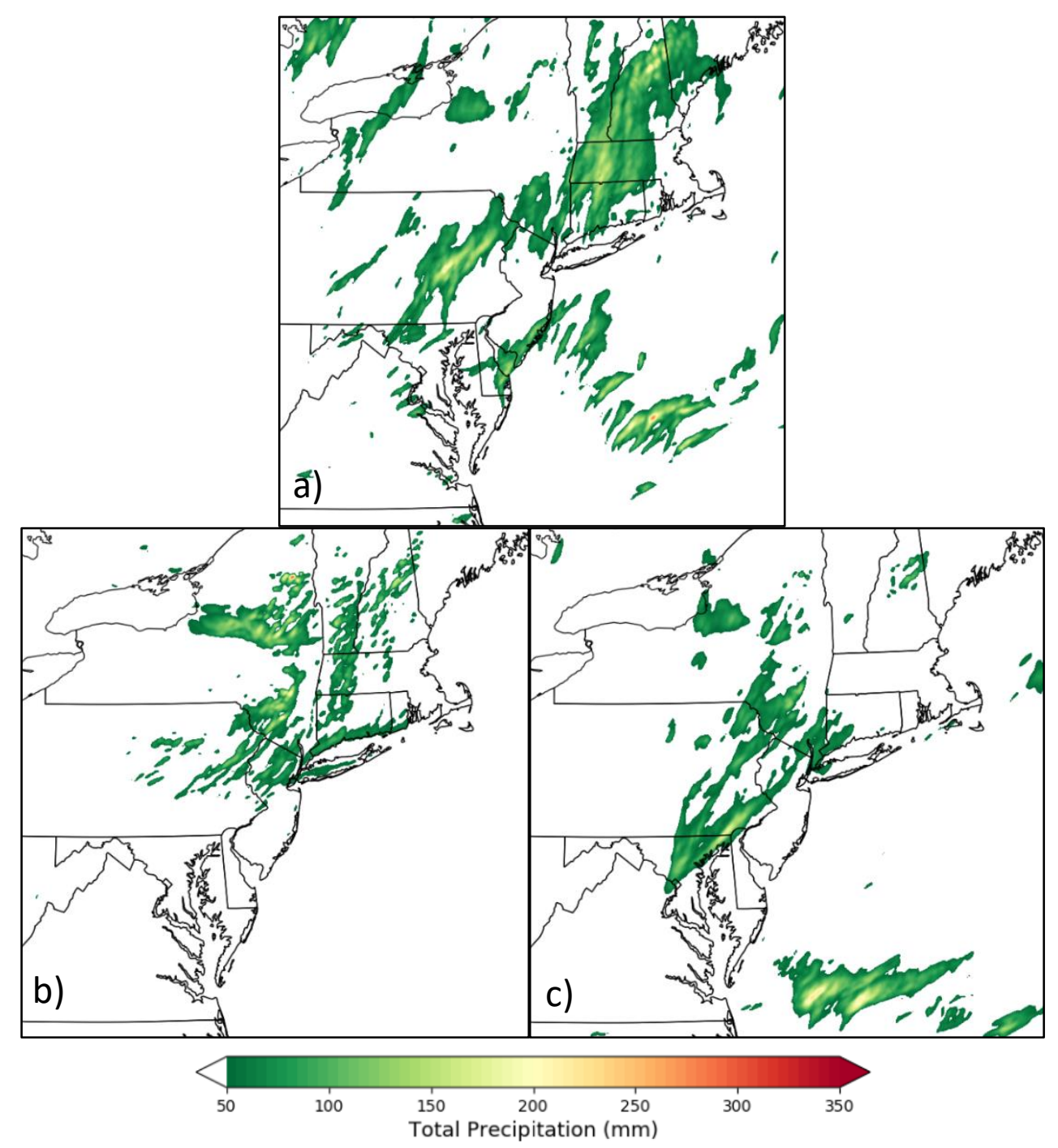

Figure 4.71. Total accumulated precipitation $(\mathrm{mm})$ from WRF domain 2 for the simulation with a) no evaporation from precipitation, b) no latent heating, and c) no surface heat fluxes.

initiated. Evaporation from precipitation would have cooled the lower levels on the cold side of the thermal boundary, increasing the temperature gradient, frontogenesis, and subsequent frontal lift.

Latent heat release also played a significant role in the total precipitation during the 2014 event. No precipitation was found offshore when latent heat release was turned 
off in the WRF simulation (Fig. 4.71b). Removing latent heating weakened the upperlevel jet off the East Coast and resulted in no upper-level divergence (not shown) over the regions of precipitation found in the control simulation. Surface temperatures remained similar between the control simulation and the simulation with no latent heating, but the trough in surface pressure shifted along the coast when latent heating was removed (not shown). The shift in lower surface pressure to the coastline was a result of the radiative heating differences of land and water. The surface pressure change and lack of latent heat release resulted in no surface low southeast of Long Island and ultimately no convection.

Surface heat fluxes aided the intense precipitation southeast of Long Island. No precipitation occurred in that area when surface heat fluxes were removed from the WRF simulation (Fig. 4.71c). The removal of surface heat fluxes also resulted in a weaker upper-level jet along the coast, lower precipitable water values over New England, cooler surface temperatures, no surface low pressure southeast of Long Island, and no frontal boundary (not shown). Removing the surface low and associated thermal boundary eliminated the lift needed to initiate convection.

\subsection{September 2010 - North Carolina}

The WRF simulation of the 2010 heavy precipitation event along the North Carolina coast resulted in two periods of intense rainfall (Fig. 4.72a) for the location of maximum precipitation just east of Myrtle Beach, off the coast of South Carolina (Fig. 4.72b). The most intense rain fell in a 3-hour period from 1800 UTC to 2100 UTC 30 September 2010 (Fig. 4.72a), but the entire rain event lasted nearly 48 hours resulting in a 


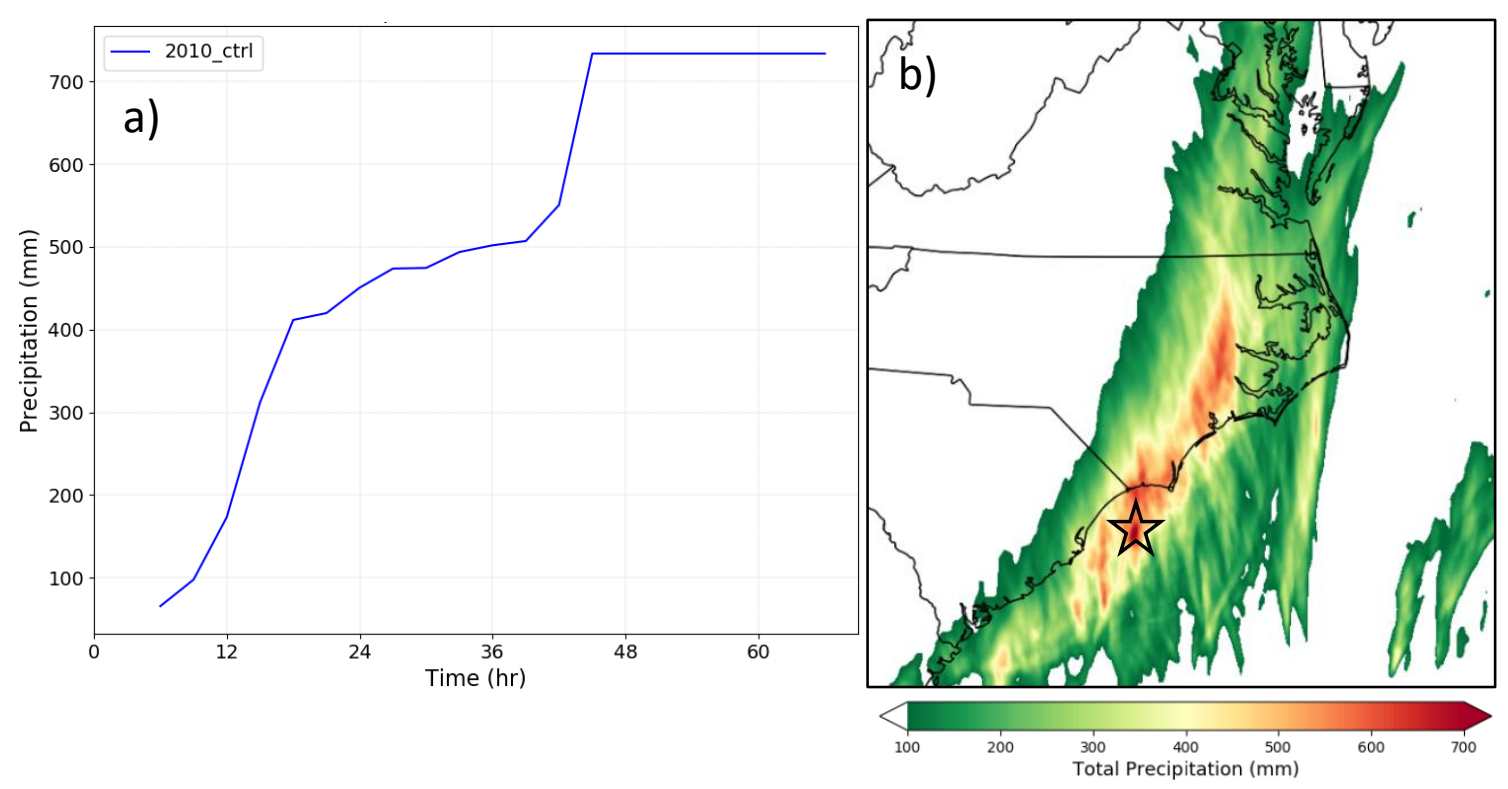

Figure 4.72. Precipitation (mm) for the 2010 event from 0000 UTC 29 September 2010 to 1800 UTC 01 October 2010 for domain 2 of the WRF control simulation a) at the location of maximum precipitation, off the coast of Myrtle Beach, South Carolina, plotted as accumulation with time and b) as the event total for the region. The transparent star represents the location of maximum precipitation.

rainfall maximum of $733.79 \mathrm{~mm}$. The precipitation field was north-south oriented along the mid-Atlantic coast with a large portion of eastern North Carolina receiving more than $500 \mathrm{~mm}$ of rain in two days (Fig. 4.72b), which was an overestimation of the total rainfall from the actual event (Fig. 2.19).

\subsubsection{Synoptic Scale}

A cut-off low was located over the southeastern United States at 1200 UTC 29 September 2010, with a north-south oriented upper-level jet stretching from Georgia to eastern Ohio before curving east across the northeastern United States (Fig. 4.73a). The 


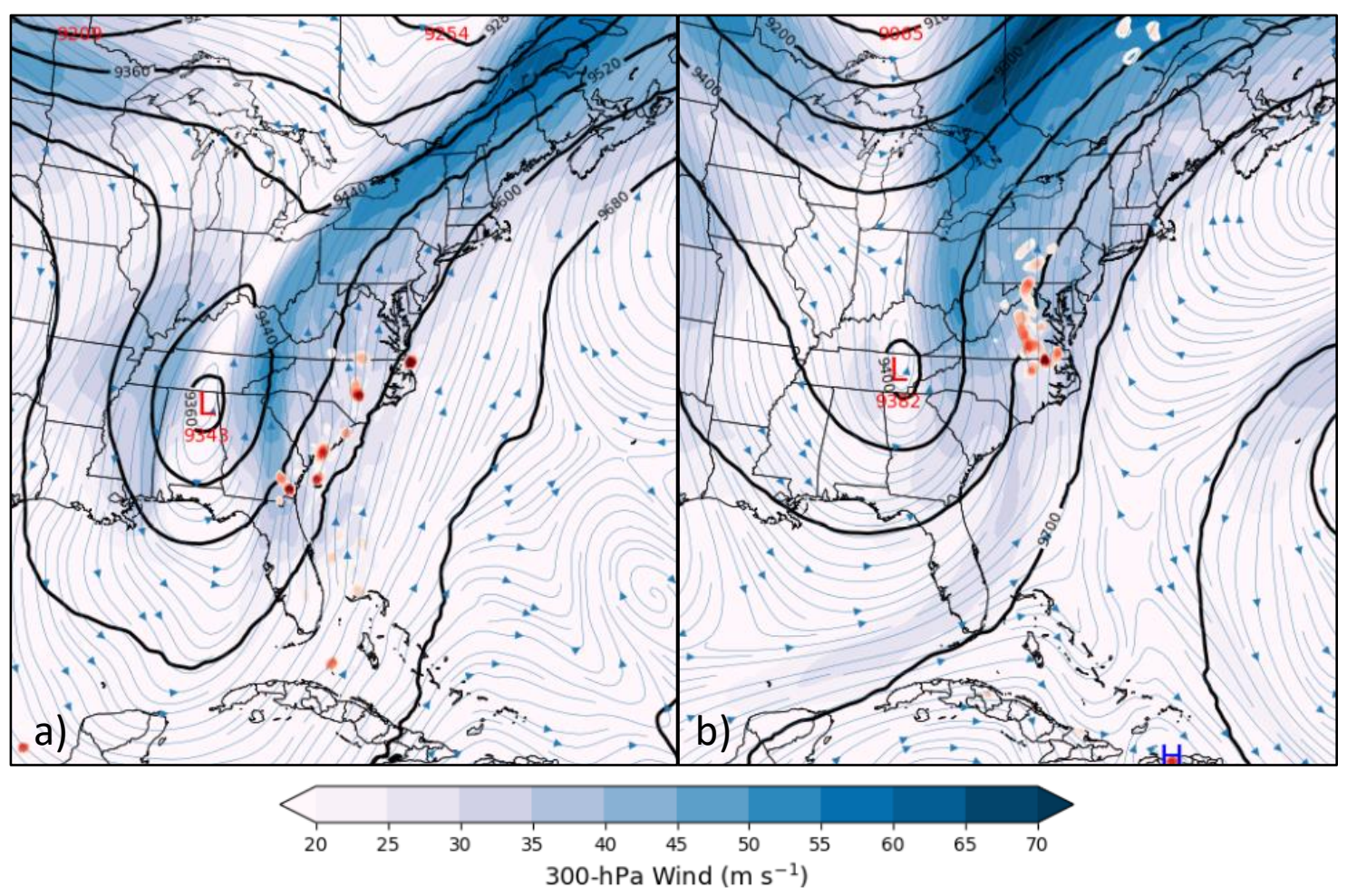

Figure 4.73. WRF domain 1300 -hPa height (gpm; thick contoured), wind ( $\mathrm{m} \mathrm{s}^{-1}$; shaded; thin streamlines), and divergence ( $10^{-5} \mathrm{~s}^{-1}$; contoured; positive red) at a) 1200 UTC 29 September 2010 and b) 0000 UTC 01 October 2010 of the control simulation.

amplified pattern placed upper-level divergence along the mid-Atlantic coast with strong southerly flow from the Caribbean. A building ridge over the western Atlantic allowed the trough of low pressure to remain over the southeastern United States through 0000 UTC 01 October 2010 (Fig. 4.73b), keeping the mid-Atlantic coast in the right entrance region of the upper-level jet for over 36 hours.

At 1800 UTC 30 September 2010, at the onset of the intense precipitation (Fig. 4.72a), an area of low pressure at $850 \mathrm{hPa}$ was located off the coast of South Carolina (Fig. 4.74a). The low-level cyclone was collocated with increased mid-level vorticity associated with the upper-level trough (not shown). The cyclonic flow created strong 


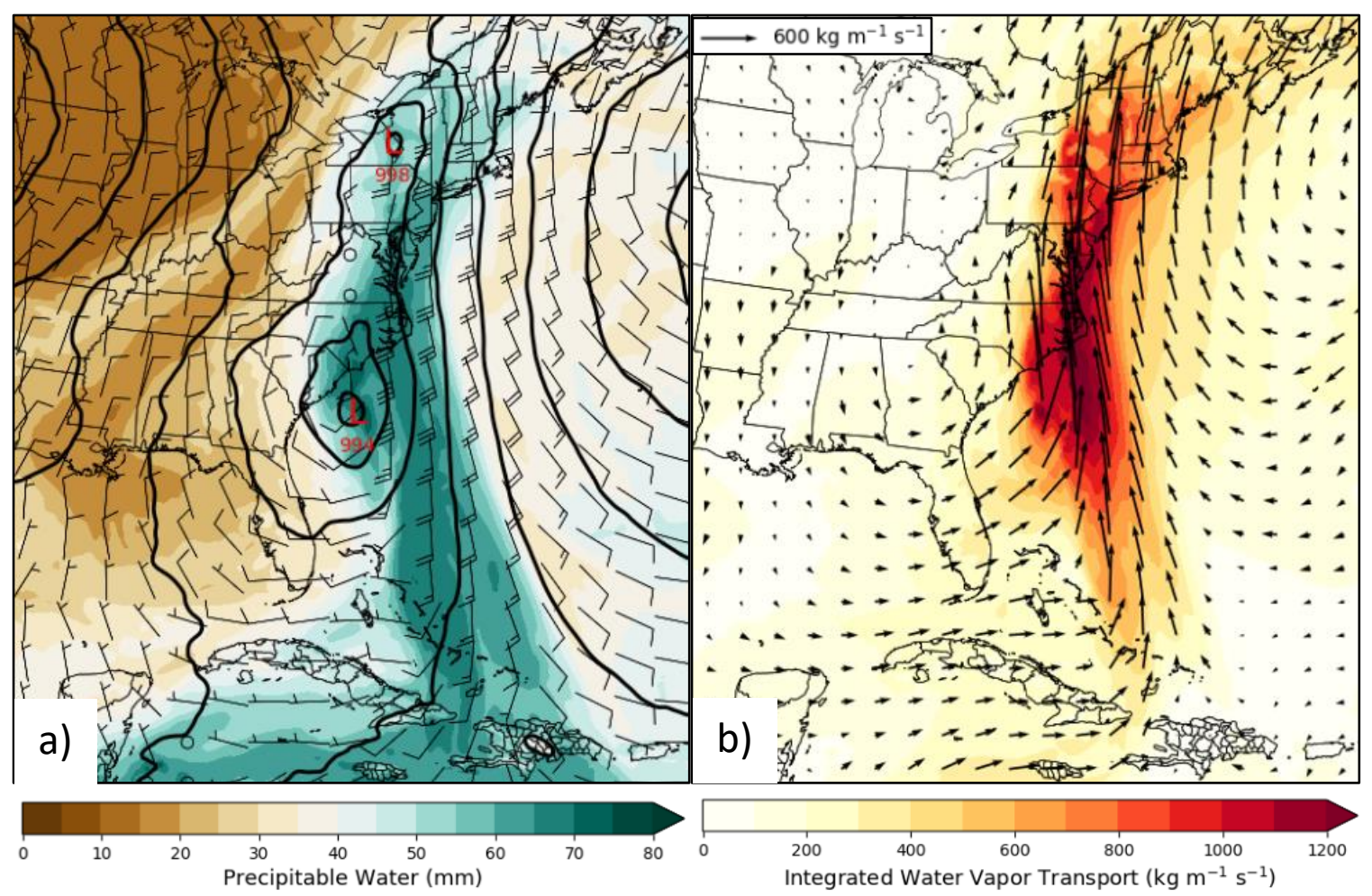

Figure 4.74. WRF results for domain 1 of the control simulation at 1800 UTC 30 September 2010 displaying a) precipitable water ( $\mathrm{mm}$; shaded), sea level pressure (hPa; contoured), 850-hPa wind ( $\left.\mathrm{m} \mathrm{s}^{-1}\right)$, and $\mathrm{b}$ ) integrated water vapor transport (IVT; $\mathrm{kg} \mathrm{m}^{-1} \mathrm{~s}^{-1}$ ).

southerly winds (up to $35 \mathrm{~m} \mathrm{~s}^{-1}$ ) at $850 \mathrm{hPa}$ off the mid-Atlantic coast (Fig. 4.74a). The deep southerly flow led to an increase in precipitable water values along the East Coast, with a moisture plume stretching from the Caribbean to New England (Fig. 4.74a). IVT values in the region of the moisture plume approached $1200 \mathrm{~kg} \mathrm{~m}^{-1} \mathrm{~s}^{-1}$ with vectors indicating that the transport originated in the Caribbean (Fig. 4.74b). The length of the plume and the intensity of IVT define this moisture plume as an atmospheric river (Mahoney et al. 2016). The highest IVT values were off the coast of the Carolinas, directed into eastern North Carolina and north along the East Coast (Fig. 4.74b), where the Gulf Stream may have contributed to the added moisture. 


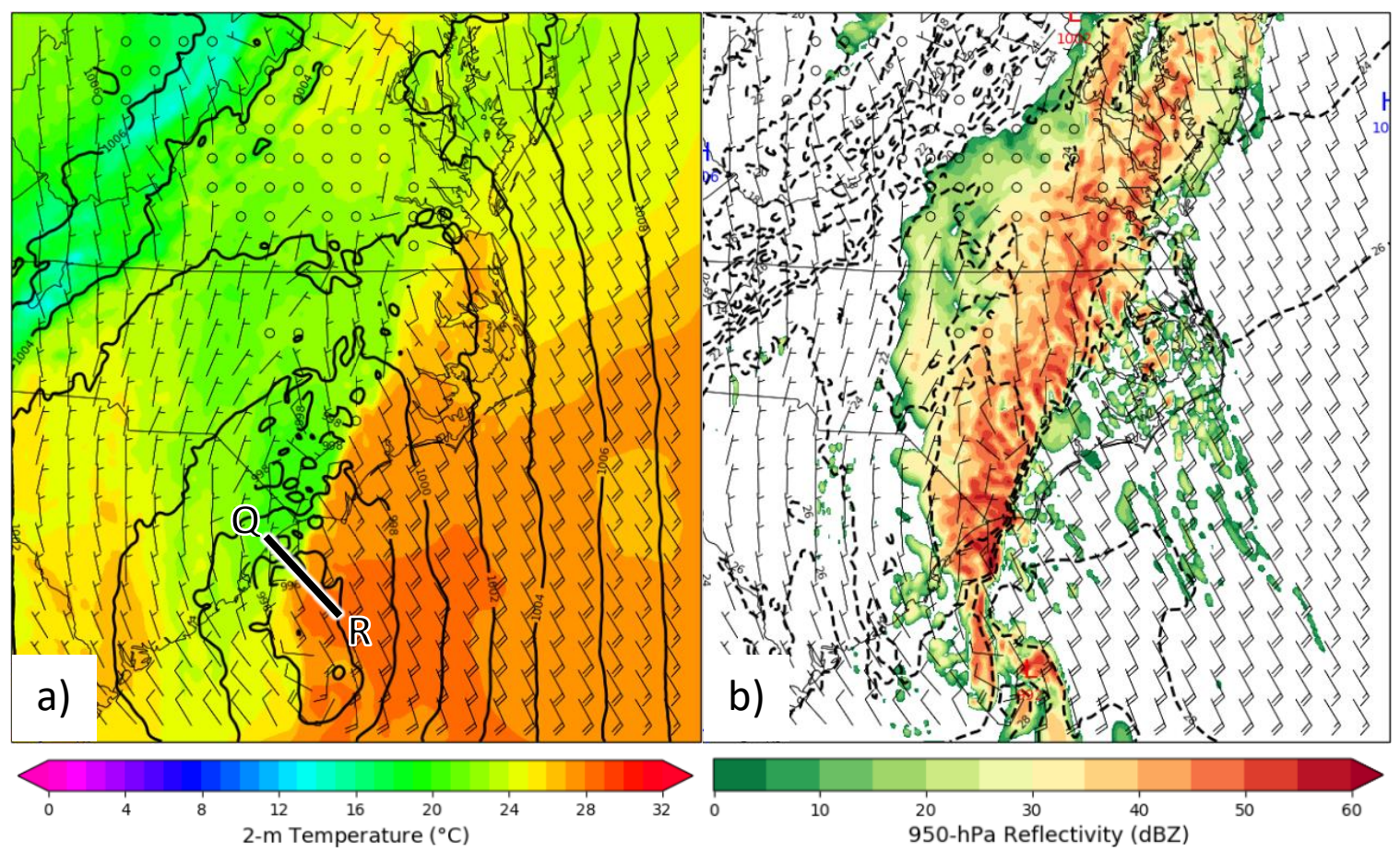

Figure 4.75. WRF results for domain 2 of the control simulation at 1800 UTC 30 September 2010 displaying $10-\mathrm{m}$ wind $\left(\mathrm{m} \mathrm{s}^{-1}\right)$, a) sea level pressure ( $\mathrm{hPa}$; contoured), 2-m temperature ( ${ }^{\circ} \mathrm{C}$; shaded), line segment $\mathrm{QR}, \mathrm{b}) 2-\mathrm{m}$ temperature $\left({ }^{\circ} \mathrm{C}\right.$; dashed), and 950-hPa reflectivity (dBZ; shaded).

\subsubsection{Mesoscale}

A surface low was also present off the coast of South Carolina at 1800 UTC 30 September 2010 (Fig. 4.75a). A strong gradient $\left(10^{\circ} \mathrm{C} 100 \mathrm{~km}^{-1}\right)$ in surface temperatures was located just north of the surface low, with the boundary extending north across eastern North Carolina. Surface winds were southeasterly at $20 \mathrm{~m} \mathrm{~s}^{-1}$ off the coast of the Carolinas, with weak $\left(\sim 5 \mathrm{~m} \mathrm{~s}^{-1}\right)$ northerly winds over much of the landmass (Fig. 4.75a). The highest reflectivities at 1800 UTC 30 September 2010 were along and west of the strong temperature gradient, with southeasterly surface flow nearly perpendicular to the frontal boundary (Fig. 4.75b). 


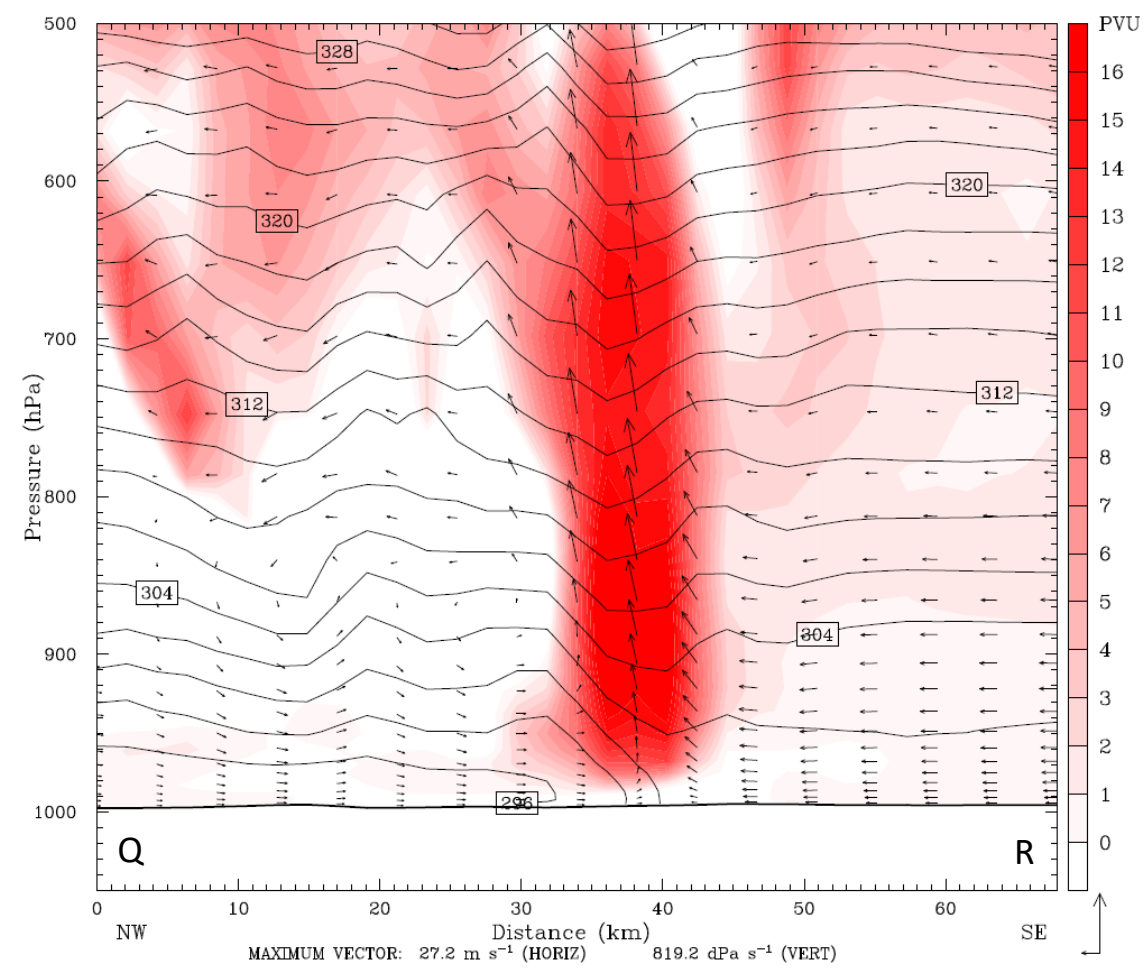

Figure 4.76. WRF domain 2 cross section from the control simulation at 1800 UTC 30 September 2010 for line segment QR (Fig. 4.75a) displaying potential vorticity (PVU; shaded red), potential temperature ( $\mathrm{K}$; contoured), and circulation vectors $\left[\mathrm{m} \mathrm{s}^{-1}, \mathrm{dPa} \mathrm{s}^{-1}\left(1 \mathrm{dPa} \mathrm{s}^{-1}=10^{-3} \mathrm{mb} \mathrm{s}^{-1}\right)\right]$.

The potential temperature gradient in the location of the boundary, where isentropes slope down to the surface, was not as strong for the 2010 event (Fig. 4.76) as it was with the 2015 event (Fig. 4.15b) at the onset of the intense precipitation. However, a boundary was still evident and was associated with a thermally direct circulation (Fig. 4.76). Strong ascent and PV were present from 950 to $500 \mathrm{hPa}$ directly above the boundary. Isentropes sloped downward in the region of the front from the surface through the mid-levels, in conjunction with the strong vertical velocities (Fig. 4.76).

The sloping isentropes and strong ascent led to a strongly negative signal in tilting frontogenesis through the mid-levels above the boundary at 1800 UTC 30 September 

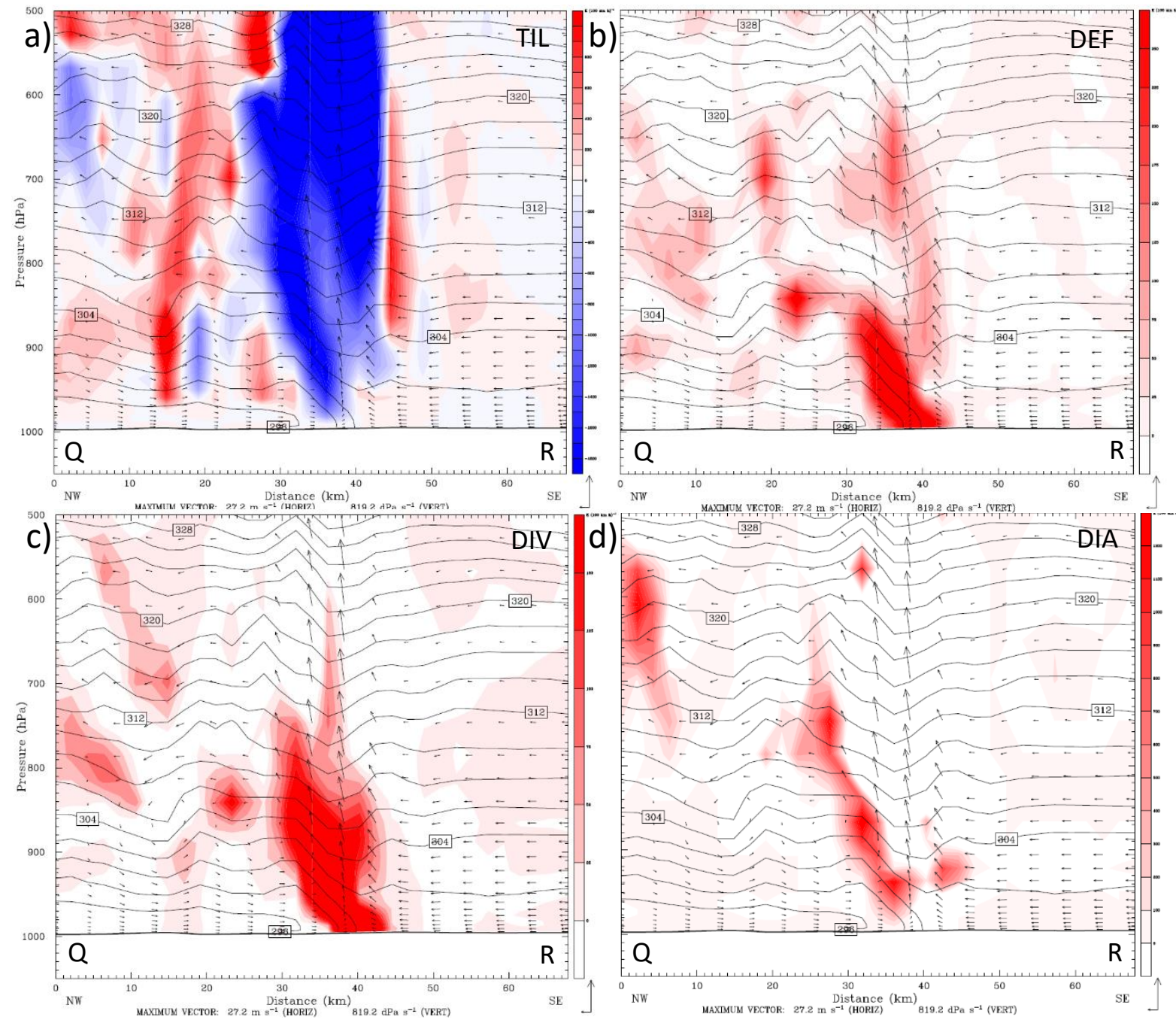

Figure 4.77. Cross sections QR (Fig. 4.75a) showing a) tilting, b) deformation, c) divergence, and d) diabatic frontogenesis [ $\mathrm{K}(100 \mathrm{~km} \mathrm{~h})^{-1}$; shaded; positive red, negative blue], potential temperature ( $\mathrm{K}$; contoured), and circulation vectors $\left[\mathrm{m} \mathrm{s}^{-1}, \mathrm{dPa} \mathrm{s}^{-1}\left(1 \mathrm{dPa} \mathrm{s}^{-1}=10^{-3} \mathrm{mb} \mathrm{s}^{-1}\right)\right.$ ] at $1800 \mathrm{UTC} 30$ September 2010 from WRF domain 2 of the control simulation.

2010 (Fig. 4.77a). As with previous events, strong deformation (Fig. 4.77b) and

divergence (Fig. 4.77c) frontogenesis were found near the surface within and just southeast of the front to support the low-level frontal circulation. However, deformation and divergence frontogenesis were strongly positive from the surface to $800 \mathrm{hPa}$, 


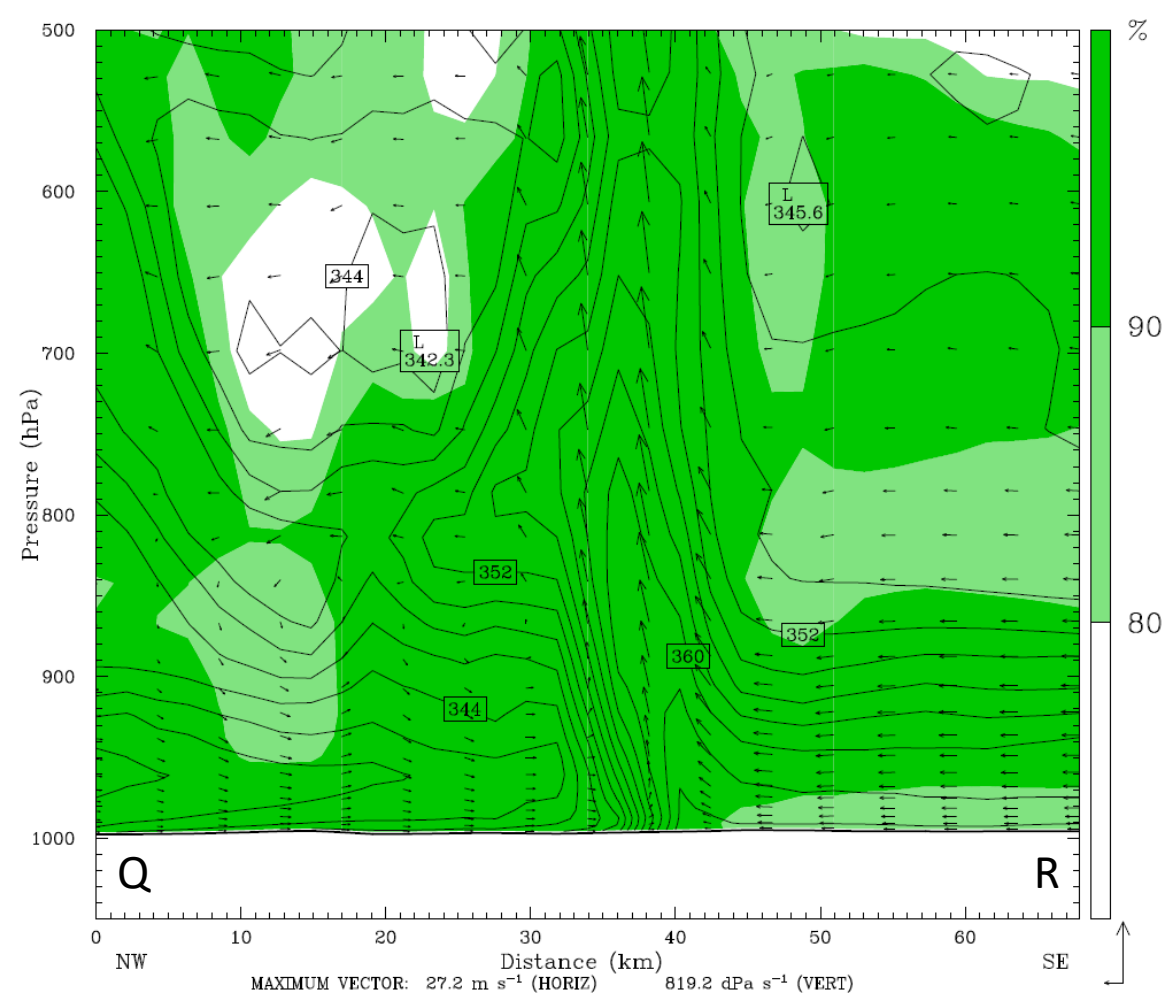

Figure 4.78. Cross sections QR (Fig. 4.75a) showing equivalent potential temperature (K; contoured), relative humidity (shaded), and circulation vectors $\left[\mathrm{m} \mathrm{s}^{-1}, \mathrm{dPa} \mathrm{s}^{-1}\left(1 \mathrm{dPa} \mathrm{s}^{-1}=10^{-3} \mathrm{mb} \mathrm{s}^{-1}\right)\right]$ at 1800 UTC 30 September 2010 from WRF domain 2 of the control simulation.

allowing the frontal circulation to extend to that level. Diabatic heating was evident with the sloping isentropes in the mid-levels and was supported by the presence of diabatic frontogenesis along the sloping isentropes from 950 to $700 \mathrm{hPa}$ (Fig. 4.77d).

The column of air in the region of the frontal boundary was saturated from the surface through $500 \mathrm{hPa}$ at 1800 UTC 30 September 2010 (Fig. 4.78). As with previous events, a layer of air with RH values greater than 90\% was found between 975 and 850 $\mathrm{hPa}$ southeast of the front in an area of southeasterly flow and PI. The saturated air was advected into the region of the frontal boundary, where PI stretched from the surface through $600 \mathrm{hPa}$ (Fig. 4.78). 


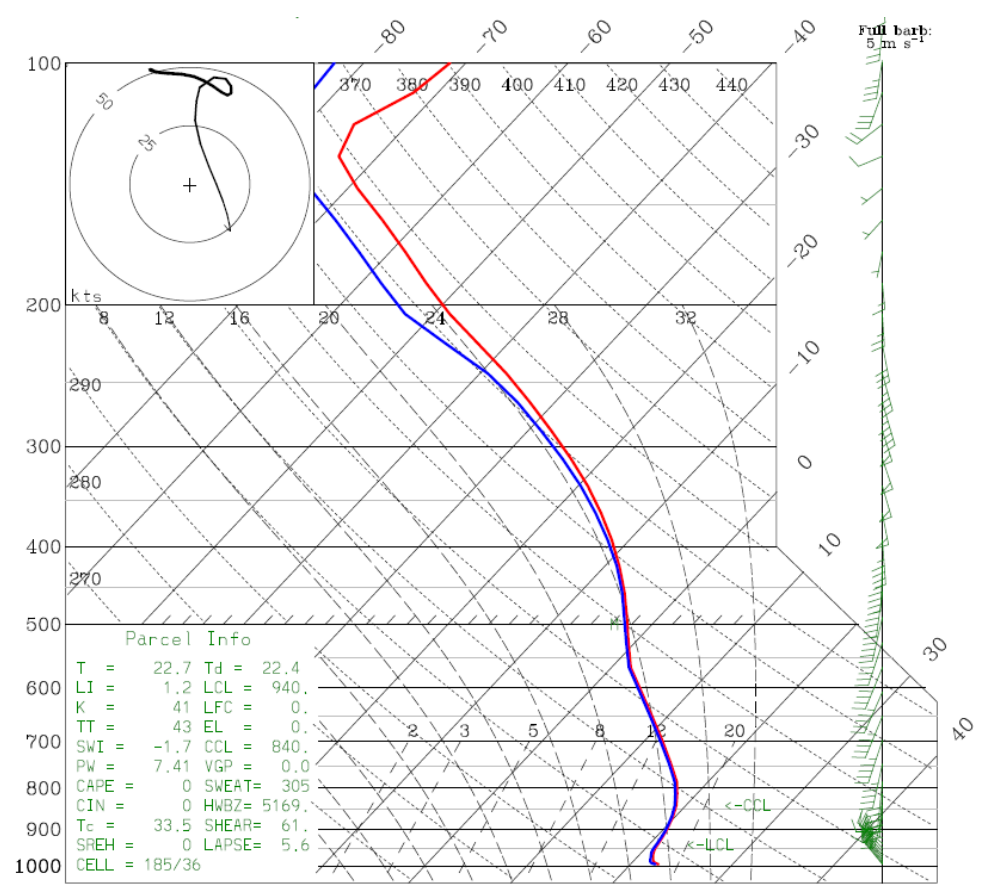

Figure 4.79. WRF domain 2 sounding from the control simulation at 1800 UTC 30 September 2010 for the location of maximum precipitation showing temperature $\left({ }^{\circ} \mathrm{C}\right.$; red), dew point $\left({ }^{\circ} \mathrm{C}\right.$; blue), and wind $\left(\mathrm{m} \mathrm{s}^{-1}\right)$.

The analysis of potential temperature in the vertical cross section identified a cooler, stable layer from the surface to $900 \mathrm{hPa}$ (Fig. 4.78). The intense convection occurred above a stable surface layer; thus, it was elevated convection. However, a clear inversion was not found in the temperature profile at the location of maximum rainfall at 1800 UTC 30 September 2010, the onset of the intense precipitation, but the environment was nearly isothermal between 950 and $850 \mathrm{hPa}$ (Fig. 4.79). Winds from the surface to $925 \mathrm{hPa}$ were northwesterly, suggesting the location to be on the cold side of the frontal boundary. The atmospheric column at the location of intense precipitation was saturated from the surface to nearly $400 \mathrm{hPa}$ (Fig. 4.79). Even though the layer from 950 to 850 hPa was saturated, the lapse rate showed that it was absolutely stable. Consistent with the 
decreasing $\theta_{e}$ values with height (Fig. 4.78), the layer between 800 and $570 \mathrm{hPa}$ was conditionally unstable and had strong south-southwesterly winds at $45 \mathrm{~m} \mathrm{~s}^{-1}$ (Fig. 4.79). The cross sections and the sounding at the time and location of intense precipitation suggest the convection was elevated.

\subsubsection{Terrain Influence}

The precipitation field had the same orientation and a similar areal extent when terrain was removed from the WRF simulation of the 2010 event (Fig. 4.80a), as compared to the control simulation. Precipitation totals decreased over eastern North Carolina and off the coast of South Carolina. The maximum accumulated precipitation in the simulation was $589.83 \mathrm{~mm}$, which was roughly $140 \mathrm{~mm}$ less than the maximum in the control simulation.

The upper-level pattern was consistent with the control simulation after terrain was removed, except that the upper-level low was able to shift south over southern Alabama (not shown) instead of north over eastern Tennessee (Fig. 4.73b) by 0000 UTC 01 October 2010. Removing terrain amplified the upper-level pattern and allowed the extratropical cyclone to deepen over the southeastern United States. The southern shift in the upper-level trough moved the $850-\mathrm{hPa}$ low southwest off the coast of southern South Carolina at 1800 UTC 30 September 2010 (Fig. 4.80b). The low-level cyclone had a lower central pressure and allowed winds off the coast of the Carolinas to become southeasterly (Fig. 4.80b) instead of southerly (Fig. 4.74a). The precipitable water pattern showed a moisture plume stretching to the Caribbean (Fig. 4.80b), with an increase in 


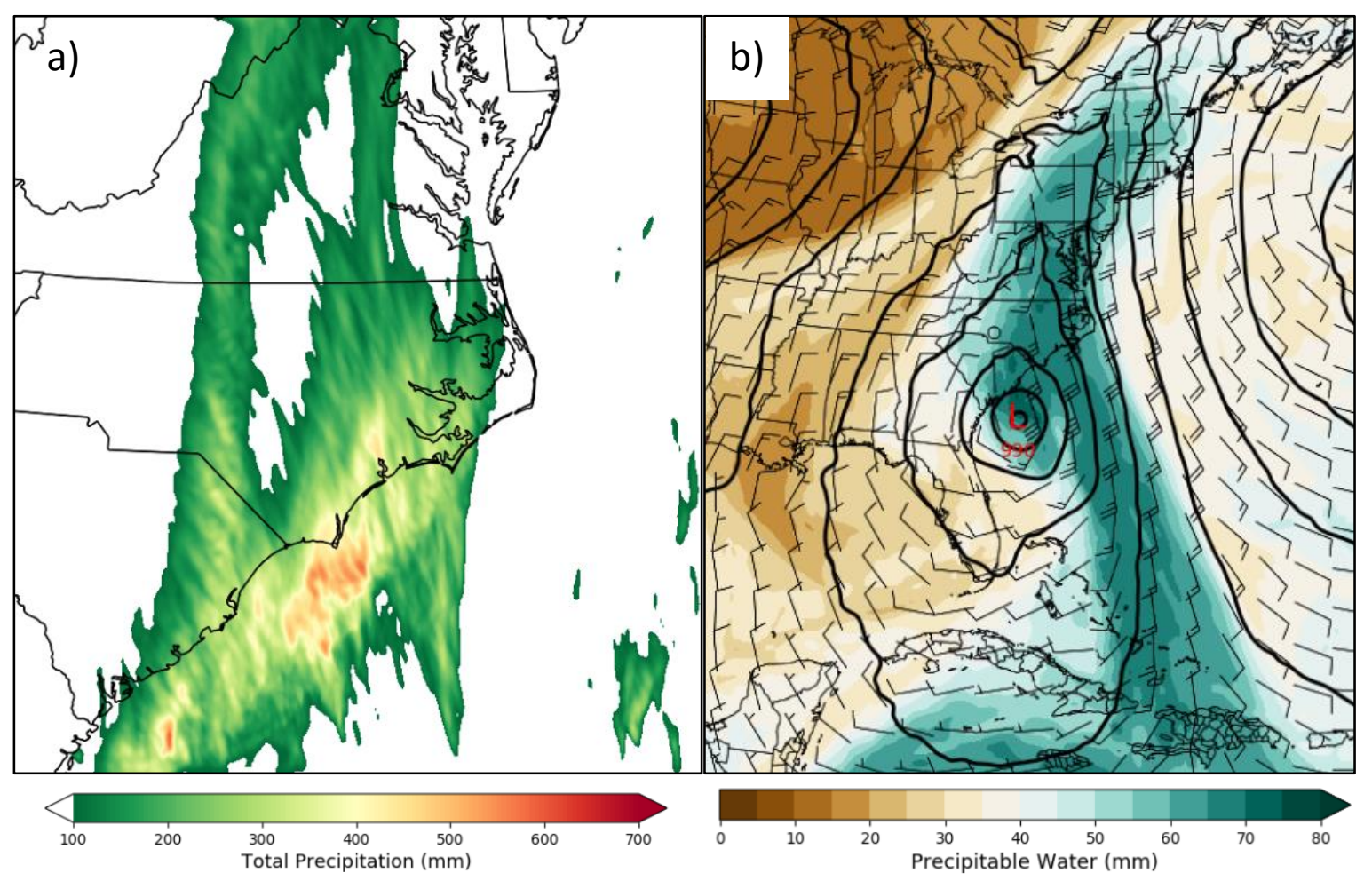

Figure 4.80. WRF results of the simulation with terrain removed displaying a) total accumulated precipitation $(\mathrm{mm})$ for domain 2 and b) precipitable water ( $\mathrm{mm}$; shaded), sea level pressure ( $\mathrm{hPa}$; contoured), and 850-hPa wind ( $\mathrm{m} \mathrm{s}^{-1}$ ) for domain 1 at 1800 UTC 30 September 2010.

moisture for New England when terrain was removed that corresponded with an increase in IVT where the northern Appalachian Mountains were located in the control simulation (not shown).

The deeper low-level cyclone off the coast of South Carolina at 1800 UTC 30 September 2010 was also seen in sea level pressure, with a surface temperature gradient stretching north across eastern North Carolina from the area of low pressure (Fig. 4.81a). The shift in surface winds was consistent with the control simulation (Fig. 4.75a) after terrain was removed (Fig. 4.81a) near the frontal boundary. The surface temperature gradient appeared the strongest near the South Carolina coast and weaker than the control 


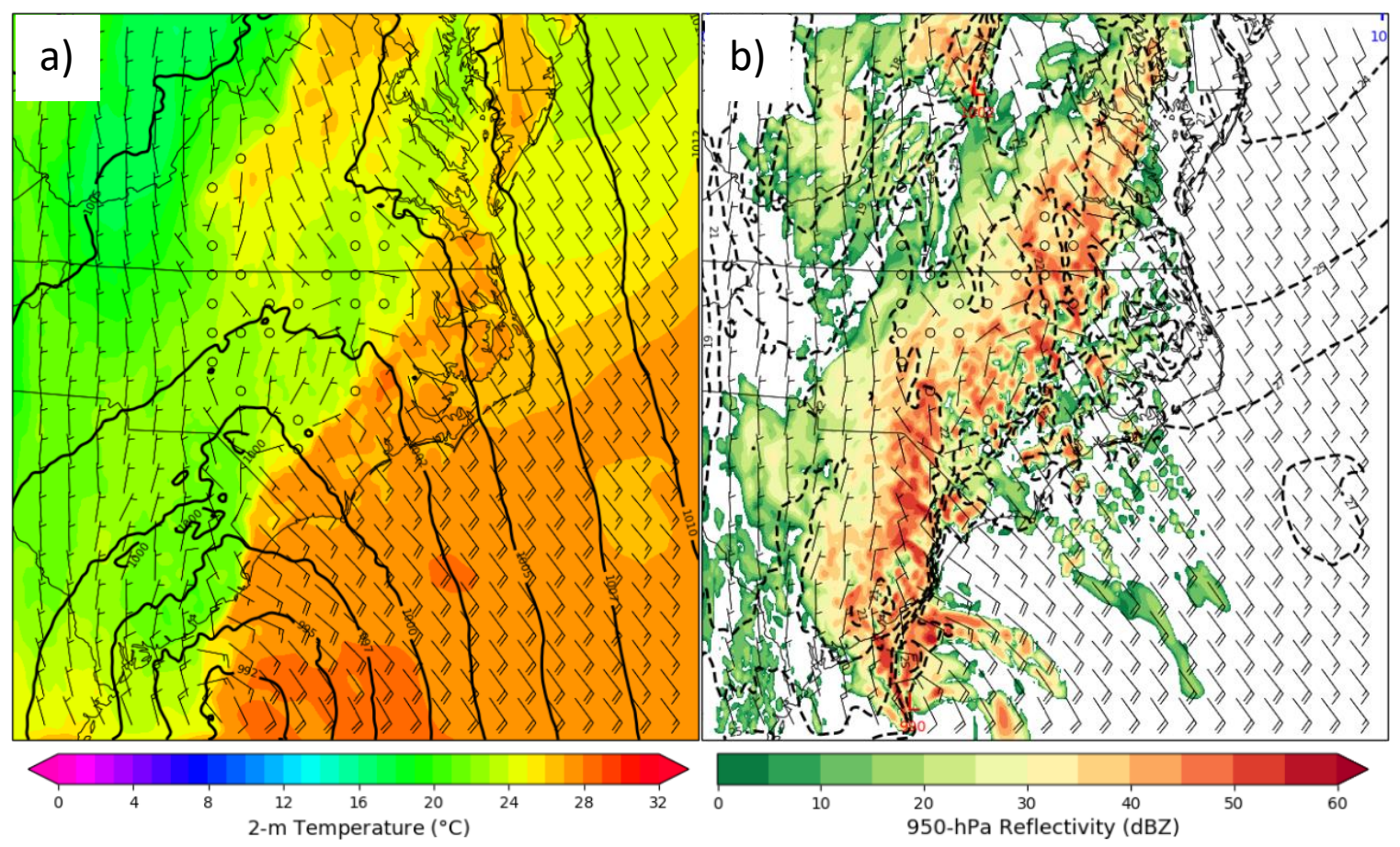

Figure 4.81. WRF results for domain 2 of the simulation with terrain removed at 1800 UTC 30 September 2010 displaying 10-m wind $\left(\mathrm{m} \mathrm{s}^{-1}\right)$, a) sea level pressure (hPa; contoured), 2-m temperature $\left({ }^{\circ} \mathrm{C}\right.$; shaded), b) 2-m temperature $\left({ }^{\circ} \mathrm{C}\right.$; dashed), and $950-\mathrm{hPa}$ reflectivity (dBZ; shaded).

simulation gradient across eastern North Carolina (Fig. 4.81a). Reflectivities also revealed less intense precipitation across eastern North Carolina with widespread precipitation across South Carolina at 1800 UTC 30 September 2010 (Fig. 4.81b), which differed from that of the control simulation (Fig. 4.75b). The orographic effects on the extratropical cyclone and coastal front contributed to the total accumulated precipitation in the 2010 event. 


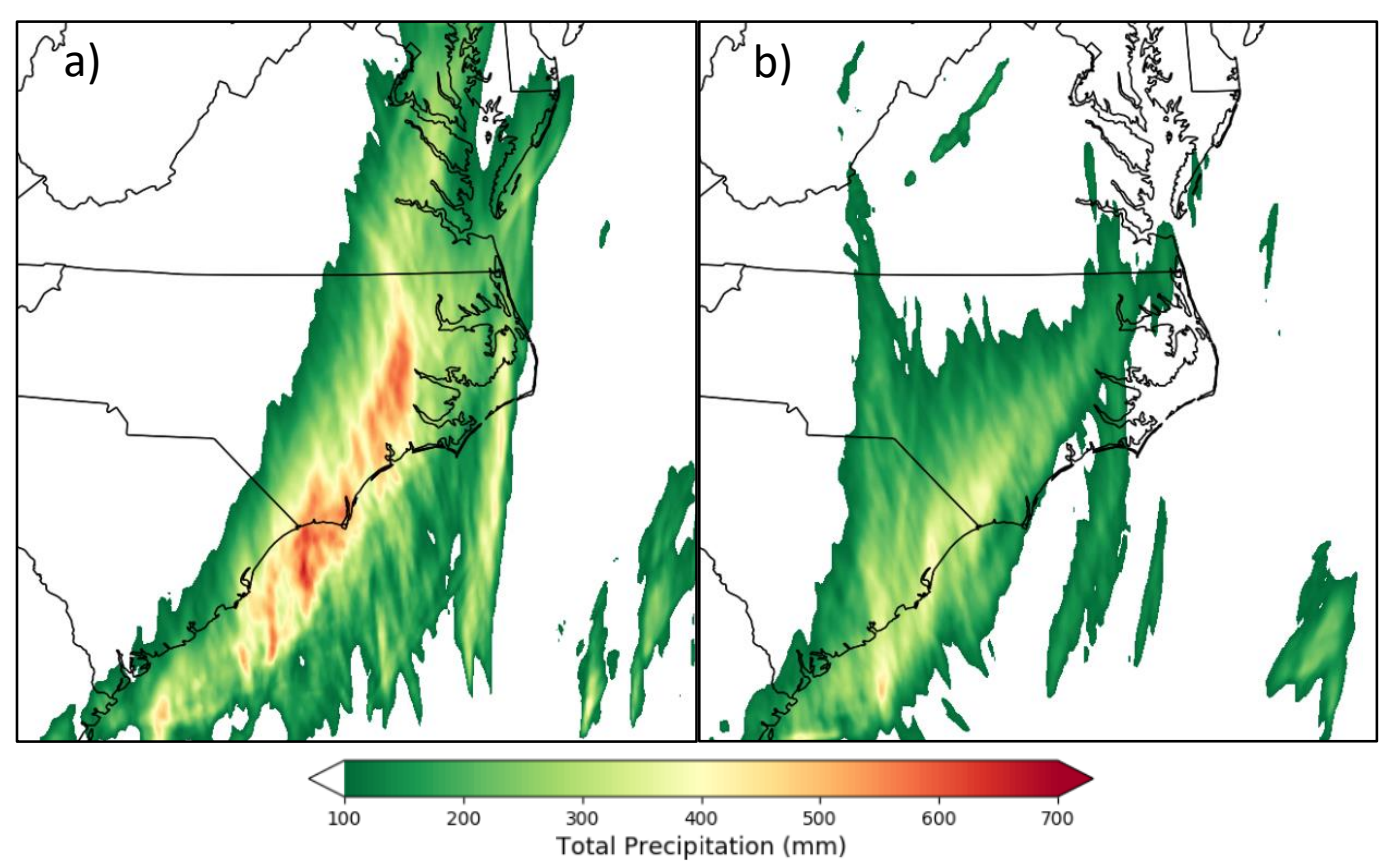

Figure 4.82. Total accumulated precipitation $(\mathrm{mm})$ from WRF domain 2 for the simulation with a) no evaporation from precipitation and b) no surface heat fluxes.

\subsubsection{Diabatic Processes}

Removing evaporation from precipitation in the WRF simulation had no noticeable impact on the 2010 intense convection event. The extratropical cyclone, moisture plume, and frontal boundary all remained consistent with the control simulation. As a result, the total accumulated precipitation field from the simulation with evaporation removed (Fig. 4.82a) appeared nearly identical to that of the control simulation (Fig. 4.72b). Removing latent heating from the WRF simulation had a major impact on the 2010 event, with less than $100 \mathrm{~mm}$ of precipitation falling over the Carolinas for the 66hour period (not shown). Surface heat fluxes had less of an impact than latent heat release. The accumulated precipitation field across eastern North Carolina and coastal 

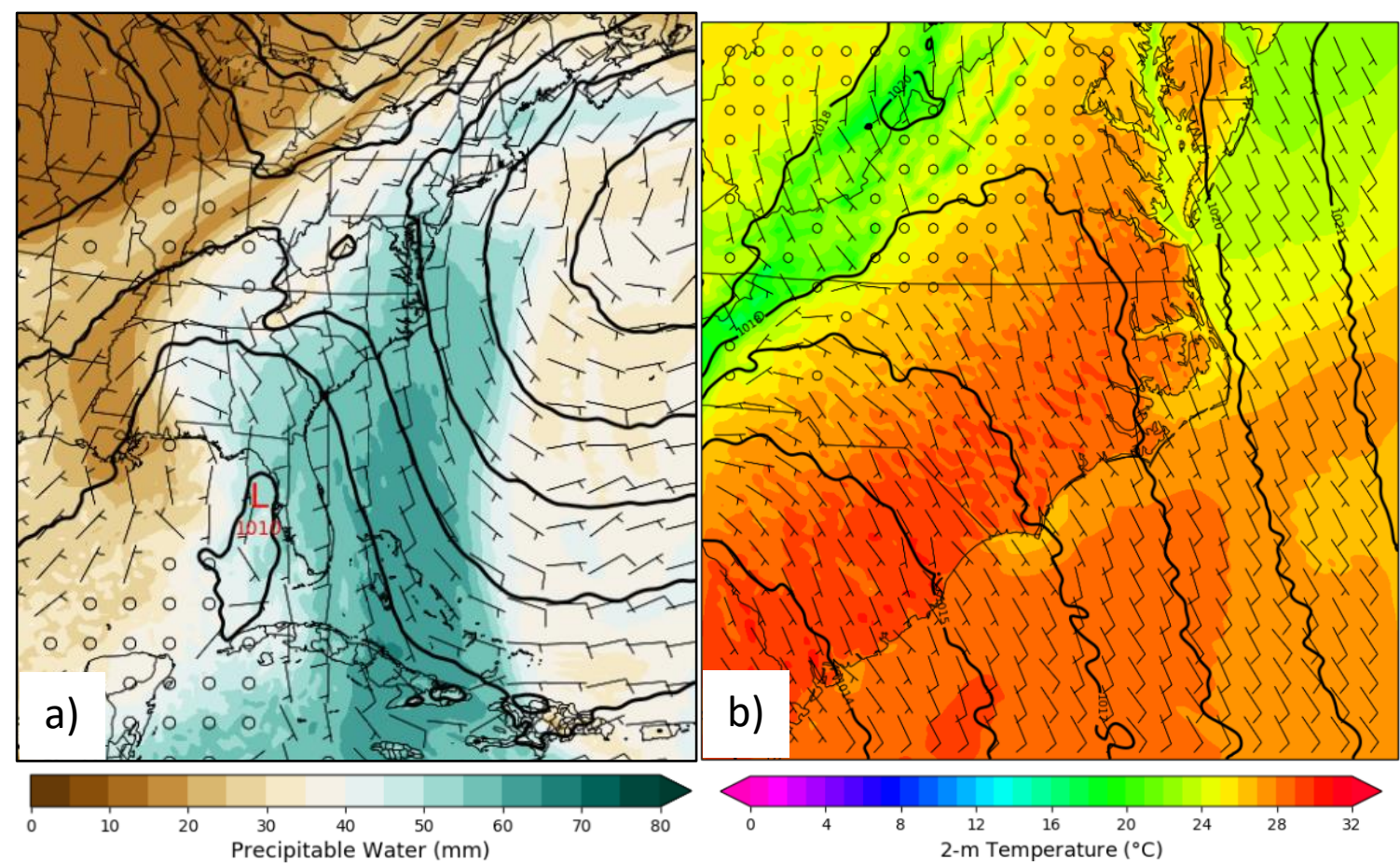

Figure 4.83. WRF domain 1 results of the simulation with no latent heating at 1800 UTC 30 September 2010 displaying sea level pressure ( $\mathrm{hPa}$; contoured), a) precipitable water (mm; shaded), 850-hPa wind ( $\mathrm{m}$ $\left.\mathrm{s}^{-1}\right)$, b) 2-m temperature $\left({ }^{\circ} \mathrm{C}\right.$; shaded), and $10-\mathrm{m}$ wind $\left(\mathrm{m} \mathrm{s}^{-1}\right)$.

South Carolina when surface heat fluxes were removed (Fig. 4.82b) was similar to the control simulation precipitation (Fig. 4.72b), except that the totals were diminished by about one third. The maximum accumulated rainfall for the simulation without surface heat fluxes was $492.60 \mathrm{~mm}, 33 \%$ less than the maximum rainfall in the control simulation.

The removal of latent heat release led to a weaker upper-level jet and very little upper-level divergence over the East Coast (not shown). There was no low-level cyclone off the coast of South Carolina and the moisture plume seen in the control simulation was no longer evident in the simulation with no latent heat release (Fig. 4.83a). The coastal 


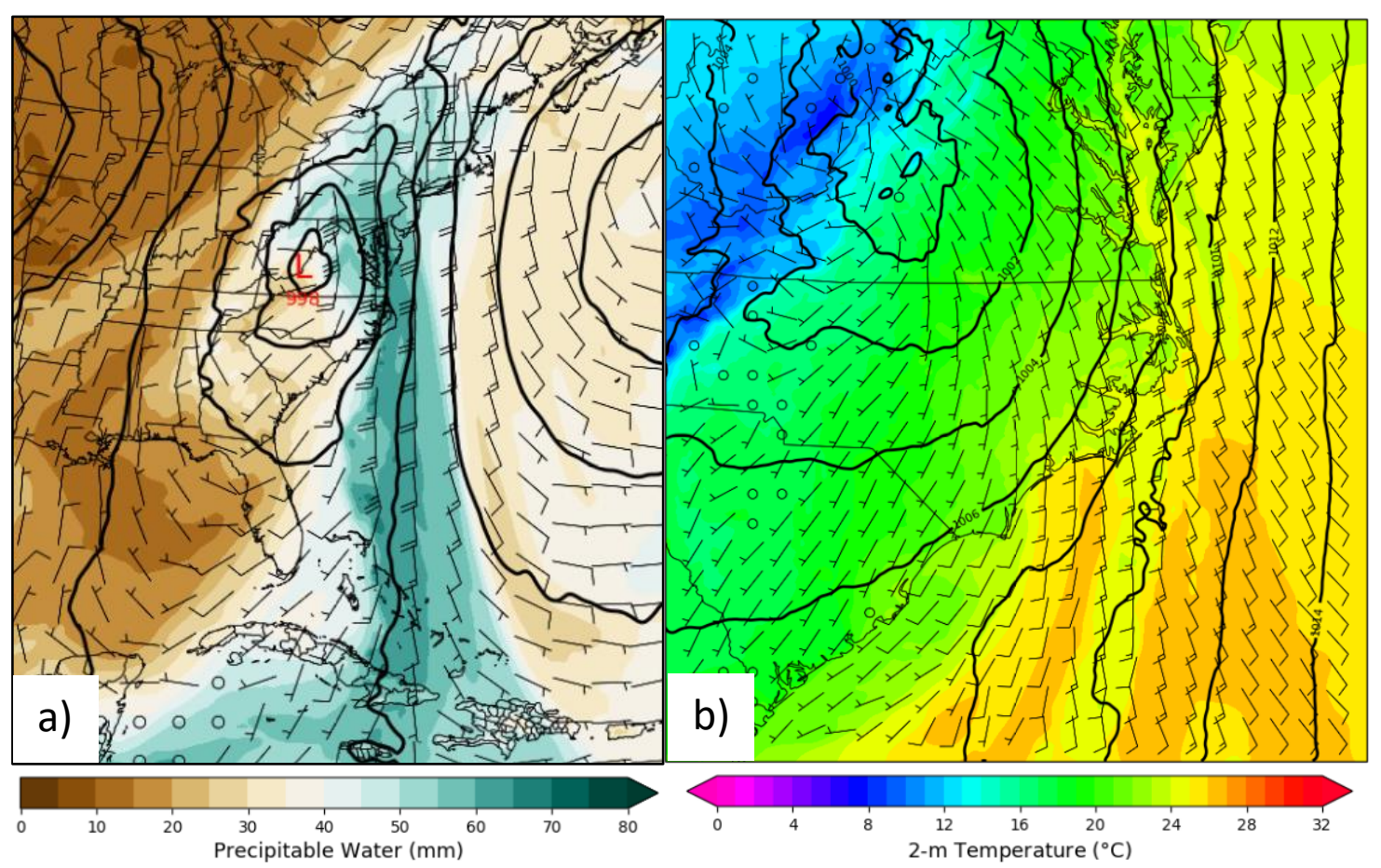

Figure 4.84. WRF results for domain 1 of the simulation with no surface heat fluxes at 1800 UTC 30 September 2010 displaying sea level pressure (hPa; contoured), a) precipitable water (mm; shaded), 850$\mathrm{hPa}$ wind $\left(\mathrm{m} \mathrm{s}^{-1}\right)$, b) 2-m temperature $\left({ }^{\circ} \mathrm{C}\right.$; shaded), and $10-\mathrm{m}$ wind $\left(\mathrm{m} \mathrm{s}^{-1}\right)$.

areas of the Carolinas still had precipitable water values above $50 \mathrm{~mm}$ at 1800 UTC 30 September 2010 (Fig. 4.83a), but those areas had values above $65 \mathrm{~mm}$ in the control simulation (Fig. 4.74a). The lack of a cyclone off the coast of South Carolina also resulted in 850-hPa southerly winds of only $10 \mathrm{~m} \mathrm{~s}^{-1}$ off the coast of the Carolinas (Fig. 4.83a). Surface winds were also weaker near the Carolinas no surface temperature gradient along the coast (Fig. 4.83b). The lack of strong southeasterly flow into the region, with no frontal boundary and no upper-level support for lift, resulted in no intense precipitation for the East Coast when latent heat release was removed from the WRF simulation. 
Surface heat fluxes had very little impact on the upper-level pattern of the 2010 event, but the 850-hPa low disappeared from the South Carolina coast at 1800 UTC 30 September 2010 when surface heat fluxes were turned off (Fig. 4.84a). The center of low pressure at $850 \mathrm{hPa}$ became centered over western Virginia. The lack of cyclonic flow south of the Carolinas caused the moisture plume to stay east of the region, lowering precipitable water values for the area (Fig. 4.84a). The surface low also shifted and resulted in weak southerly (southwesterly) flow across the majority of North Carolina (South Carolina; Fig. 4.84b). No sharp temperature gradient or wind shift was found at the surface across eastern North Carolina and no inverted trough in sea level pressure (Fig. 4.84b) to suggest the presence of a coastal front at 1800 UTC 30 September 2010.

The analyzed time was concurrent with the onset of the most intense precipitation in the control simulation. The differences found in the simulations at that time suggest no intense precipitation occurred in the simulation with no surface heat fluxes. The accumulated precipitation in the simulation with surface heat fluxes removed (Fig. 4.82b) fell earlier in the simulation when a coastal front was present near the Carolinas, a surface low was located off the coast of Georgia, and the position of the upper-level low created a region of upper-level divergence along the East Coast (not shown). The earlier precipitation was consistent with that of the control simulation (Fig. 4.72a). Surface heat fluxes only had an impact on the most intense precipitation period during the event, likely due to the impact on the coastal front. 


\section{CHAPTER 5. CONCLUSIONS}

\subsection{Summary}

Five complex meteorological events involving intense precipitation and flooding along the East Coast of the United States during fall months were analyzed in this research to better understand the cause of the significant rain accumulation. Two of the five events occurred while tropical cyclones were located offshore and the remaining three events appeared to have no correlation to a tropical cyclone. The initial motivation of this research was to determine how much influence the tropical cyclone had on the ensuing precipitation. Thus, all five events had similar atmospheric environments involving extratropical cyclones and coastal fronts. Through numerical weather prediction experiments, this research examined and quantified the role of each atmospheric feature involved in the events.

The events in this study had a combination of features that were found in Maddox "Synoptic" and "Frontal" type flood events (Maddox et al. 1979); a plume of deep tropospheric moisture was nearly perpendicular to and directed at a frontal boundary, both of which were located east of an upper-level trough, and intense precipitation occurred on the cold side of the front. Three of the five events (2010, 2011, and 2015) had stagnant atmospheric conditions resulting in rainfall that totaled between 350 and 735 mm over a period of days in their WRF control simulations, in part due to impacts on the large-scale flow by the convective processes, similar to earlier events studied by 
Schumacher and Davis (2010). However, all events had periods of intense precipitation resulting in more than $250 \mathrm{~mm}$ of rain in less than 24 hours.

The upper-level low position in each event placed the right entrance region of the upper-level jet over the area of precipitation, providing upper-level support for ascent through divergence aloft. Additionally, the upper-level flow associated with the upperlevel trough/cut-off low contributed to the amount of moisture being advected into the region of intense rain. The upper-level patterns for the events were slow moving due to high pressure over the western Atlantic, which is commonly referred to as the Bermuda High in the fall months. To further inhibit downstream motion of the upper-level trough, the latent heat release associated with the convection in the 2015 South Carolina event led to negative PV advection by the irrotational wind. The positive feedback system enabled the convection to continue for a prolonged period over the same location.

Low-level cyclones were associated with the upper-level troughs over the eastern United States during all events. The collaborative flow at all levels allowed for deep tropospheric moisture to funnel into the regions of intense precipitation. The tropical cyclone events (2011 and 2015) experienced more concentrated moisture plumes in part because of an axis of dilatation associated with a deformation zone between the extratropical cyclone and the tropical cyclone. With the 2015 event, in particular, the tropical cyclone adjusted the flow of moisture enough to prolong the rainfall by 6 hours, but ultimately limited the total amount of moisture that could be advected into the region. Additionally, the deformation zone impacted the upper-level low and associated jet streak, shifting the area with upper-level support for ascent and adjusting the precipitation field. The 2011 event experienced the same increase in total accumulated precipitation 
when the tropical cyclone was removed, but appeared to have no impact on the upperlevel jet.

The moisture plumes for each event were located over the Gulf Stream, likely increasing the available moisture for convection. Each event appeared to have moisture plumes originating from a tropical location, such as the Gulf of Mexico, the Caribbean, or the southwestern Atlantic Ocean. It did not appear to matter where the moisture came from or if a tropical cyclone was nearby, the deep tropospheric flow pattern was persistent for long enough to allow the precipitation areas to be inundated with significant moisture from the warm tropical waters.

Frontal boundaries and frontogenesis differed for each event. Only the 2011 event appeared to be associated with orographic lift, but mid-level frontogenesis may have still played a role in the convective intensity. The convection in the remaining events was initiated by frontal boundaries lifting parcels near the surface to the level of free convection, in association with strong deformation and divergence frontogenesis strengthening the thermally direct circulation and releasing gravitational instability. The front position was directly correlated with the location of convection. The Appalachian Mountains impacted the precipitation fields because of the influence on surface temperature gradients and extratropical cyclone positions, suggesting both the front and cyclone to be the main contributors to the precipitation events.

Evaporation from precipitation had little impact on total accumulated precipitation in each event. The totals were only slightly reduced $(\leq 25 \%)$ due to a decrease in relative humidity in the area of convection. Removing latent heat release resulted in little to no precipitation for each event simulation, suggesting the process to be 
a large contributor to the event. Latent heat release impacted the convection in each event through several means: negative PV advection by the irrotational wind slowed the propagation of the upper-level low, prolonging the upper-level support for ascent; isentropic surfaces sloped down above the frontal boundary in response to the mid-level heating, strengthening vertical velocities; and on a large scale, latent heat release was responsible for the location of the extratropical cyclone, the existence of the frontal boundary, and the increased moisture available to the region in the WRF simulation. Frontal boundaries were much weaker or did not exist at all when surface heat fluxes were removed from the simulations, with total accumulated precipitation in the events decreasing by at least one-third. The diabatic/precipitation processes played a role in the heavy precipitation, as expected, but mainly through impacts on the thermal boundary needed to initiate lift.

In all five events, convection occurred between 900 and $750 \mathrm{hPa}$ above a cooler, stable surface layer. Thus, all events experienced elevated convection, a known contributor to the intensity of precipitation (Rochette and Moore 1996). Dynamic processes and thermodynamic conditions needed for precipitation coexisted in each event, as expected in the fall months, leading to the significant rainfall totals and high intensity. The extratropical cyclone provided the moisture and supported ascent, while the front provided the lift needed to release instability and initiate convection. The diabatic heating from convection created a positive feedback system to strengthen the ageostrophic frontal circulation and inhibit large-scale motion, prolonging the intense precipitation for the region. The copious amounts of precipitation were also possible because the large-scale flow and warm sea-surface temperatures in the fall months 
provided ample moisture for a concentrated plume to develop and propagate over the frontal boundary.

\subsection{Synthesis}

The investigation into all five deep convection events along the East Coast revealed similarities in moisture and instability, but differences in the source of lift. All areas of intense precipitation occurred downstream of an upper-level trough or cut-off low, near the right entrance region of the upper-level jet. The upper-level trough alone allowed for southerly/southeasterly flow into the area of precipitation, but the upper-level pattern for each event also involved an area of high pressure over the Atlantic to aid the southeasterly flow. That area of high pressure was persistent for several days during each event and likely qualified as a blocking anticyclone (Lupo and Smith 1995). For 3 of the 5 events, that region of high pressure contributed to the stationarity of the upper-level flow, allowing the area of extreme precipitation to remain just downstream of the trough in a region of significant moisture, instability, and intense convection for several days. For all five events, the large-scale environment provided substantial amounts of moisture transport into the region of intense precipitation.

Removing the tropical cyclone in two of the events resulted in an increase in precipitation totals due to an increase in moisture in the region of intense precipitation and a shift in the location of precipitation due to a change in the synoptic flow pattern. The tropical cyclones impacted the precipitation event by altering the large-scale flow of moisture, deterring some of the flow and limiting the moisture availability to the region 
of heavy rain. The impact of the moisture flow on the precipitation amount and location highlighted the importance of the synoptic environment to the significance of the event.

The coastal front was present along the coast of the Carolinas in 3 of the 5 events, contributing to the convective initiation by lifting parcels to the level of free convection. Of the other two events, one was simulated well off the coast of the United States near an area of low sea level pressure with strong mesoscale forcing for ascent and the other was over central Pennsylvania where orographic lift contributed to the convection. Since all 5 events had varying magnitudes and sources of lift, it was determined that the coastal front was not the main contributor to the extreme precipitation in all of the events.

The deep tropical moisture plume collocated with the region of intense precipitation was a common factor in all five of the events analyzed. Each of the five intense rainfall events resulted from significant amounts of tropical moisture being advected into a region of elevated instability. As with PREs, only weak forcing for ascent is necessary to initiate convection when tropical moisture is in the presence of instability (Cote 2007). Therefore, the source and magnitude of ascent could be different (i.e., frontogenesis, geostrophic deformation, orography, etc.) and the result of intense precipitation could remain. For these five cases, the large-scale environment was responsible for the moisture transport poleward, contributed to the mesoscale boundaries, and aided the development of surface lows near the regions of heavy rain. Thus, the position of the upper-level low and the blocking ridge downstream were the necessary, most influential, and main contributors to each of the five events investigated. 


\section{APPENDIX A.}

Each event in this research required a slightly different WRF configuration to obtain an acceptable simulation of the heavy rainfall. All cases proved to be sensitive to the location and amount of precipitation. As discussed in Chapter 3, a sensitivity test was completed to determine which options in WRF would provide the best representation of the precipitation field for each event. The sensitivity test for the South Carolina flood of 2015 required the most attention because WRF was very sensitive to the track and strength of Hurricane Joaquin, which proved to be a factor in the location of the moisture plume and subsequent rainfall. The WRF configuration that resulted in the most accurate precipitation field (section 3.1.2), according to the 4-km NCEP Stage IV reanalysis data, was a starting point for the sensitivity tests of the remaining events due to the similarities of all events.

All additional events required less effort in obtaining a qualifying control simulation of each event. Only domain size and location, model start time, and parameterization schemes were experimented with for the remaining events; initialization data, grid resolution, and vertical levels were consistent with that used for the 2015 South Carolina flood (section 3.1.2). The initial goal of the sensitivity tests was to find a simulation acceptable if the verification resulted in an interest value of 0.90 or above. The verification method involving the MODE tool inside the MET package is described in

Chapter 3. Based on MODE results from all event simulations, the WRF configuration found to provide the most realistic simulation of the South Carolina 2015 flood was found to provide the most realistic simulation for all five events. This is no surprise, as 

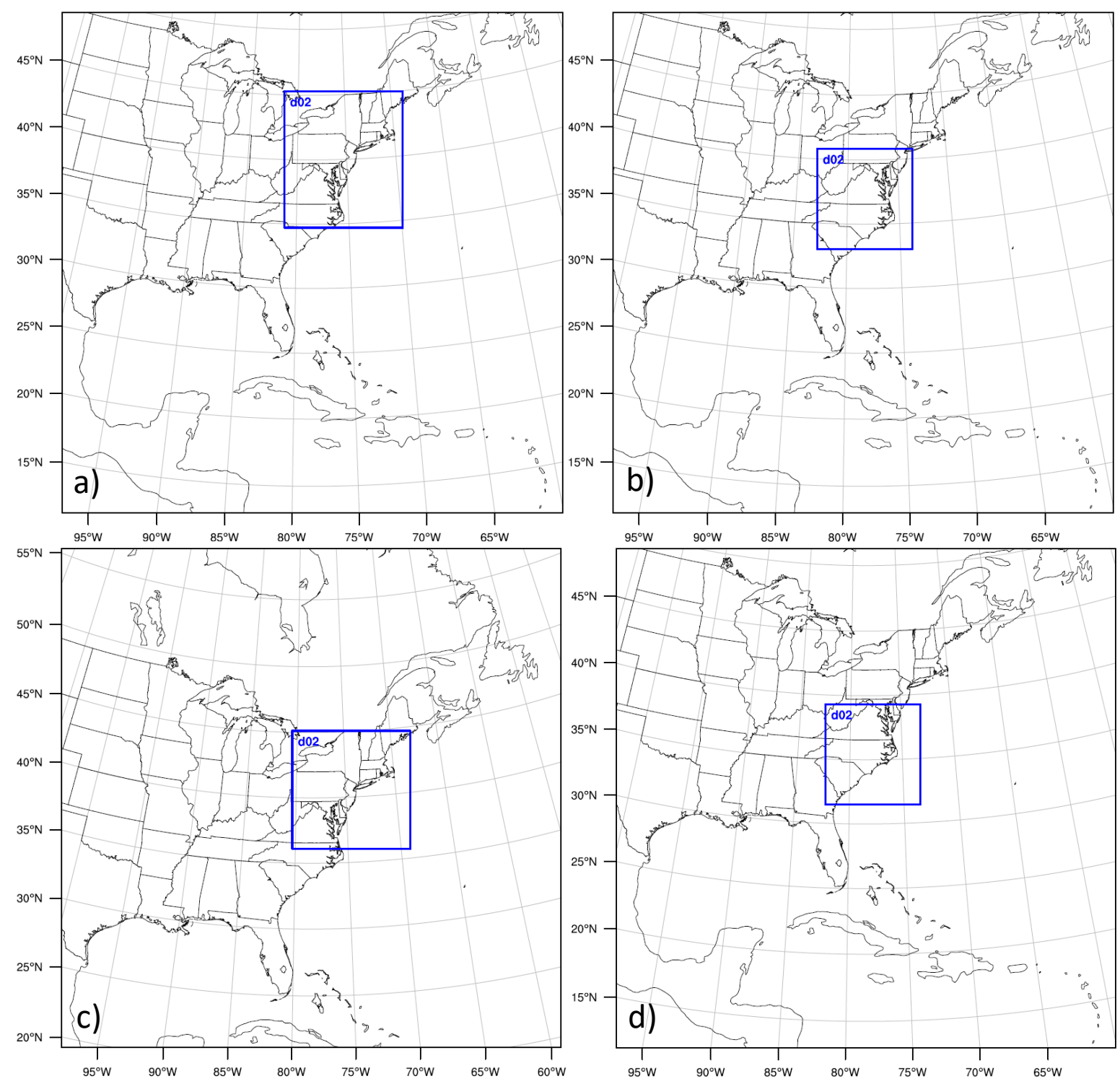

Figure A.1. WRF model domains representing the outer domain (domain 1) of 15-km grid spacing with the inner domain ( $\mathrm{d} 02$ ) of 3-km grid spacing outlined in blue for use in simulating the heavy precipitation in a) central Pennsylvania and New York in September 2011, b) North Carolina in September 2016, c) Islip, New York in August 2014, and d) North Carolina in September 2010.

the events have similar atmospheric conditions, precipitate near the coast, and occur in late summer/early fall. The WRF domain configurations for all events are displayed in Figure A.1, except that of the 2015 South Carolina flood (Fig. 3.1). 


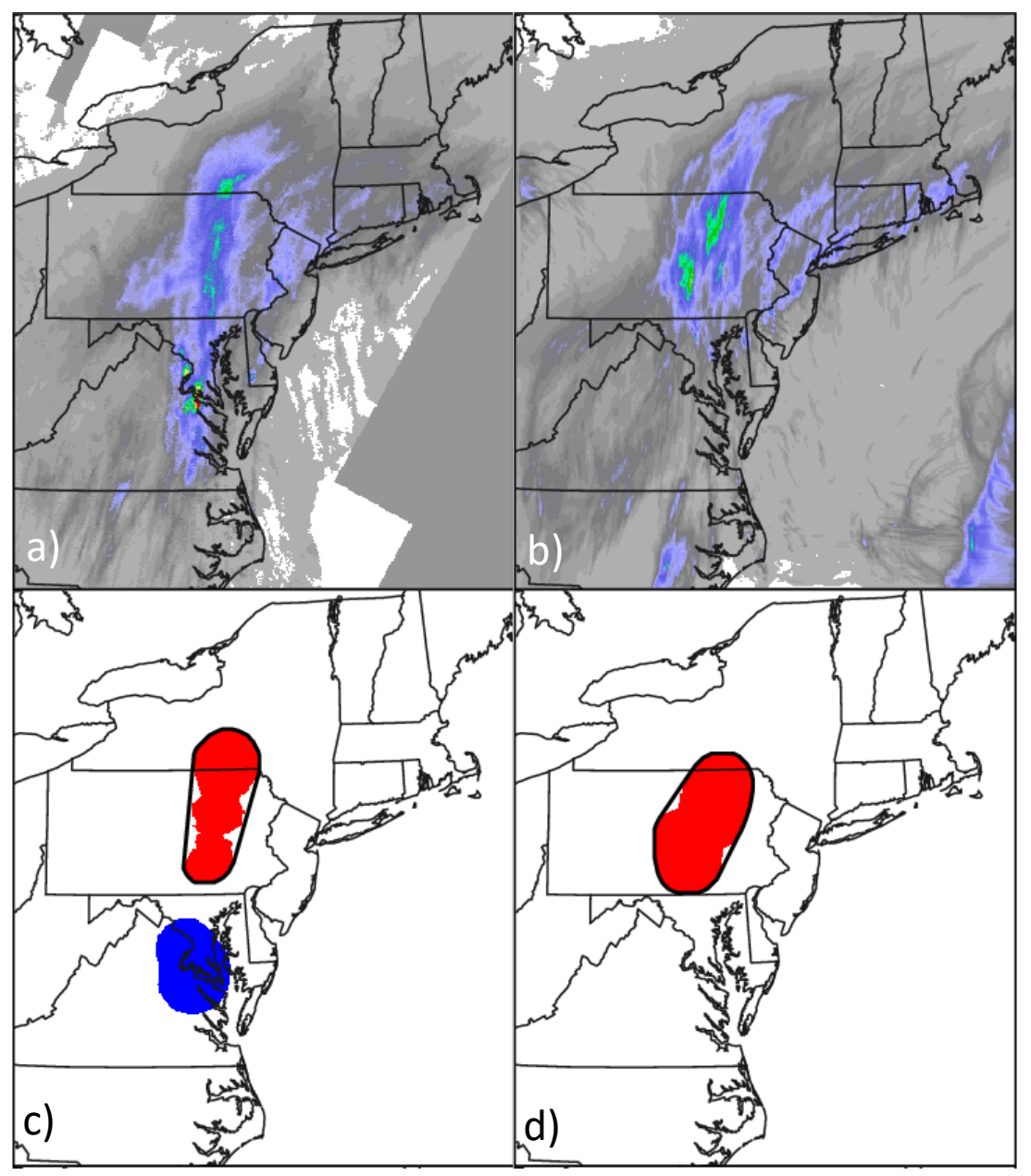

Figure A.2. Precipitation amount for the simulation of the Pennsylvania/New York heavy rain event from 1200 UTC 06 September 2011 to 1200 UTC 09 September 2011 created using a) 4-km NCEP Stage IV reanalysis data from Earth Observing Laboratory (EOL) and b) WRF control simulation output for domain 2. The MODE results are shown to identify an object (red) for c) the reanalysis data shown in a) and d) the WRF data shown in b) that are corresponding in both analyses based on a threshold of $254 \mathrm{~mm}$. The total interest value calculated in MODE for the objects (red) shown in c) and d) is 0.998 .

The 2011 flood event in central Pennsylvania and New York, involving Hurricane Katia, was simulated from 1200 UTC 06 September 2011 to 1200 UTC 09 September 2011 using the domains found in Figure A.1a. This 2011 control simulation had the most realistic precipitation results out of all events with an interest value of 0.998 (Fig. A.2). 

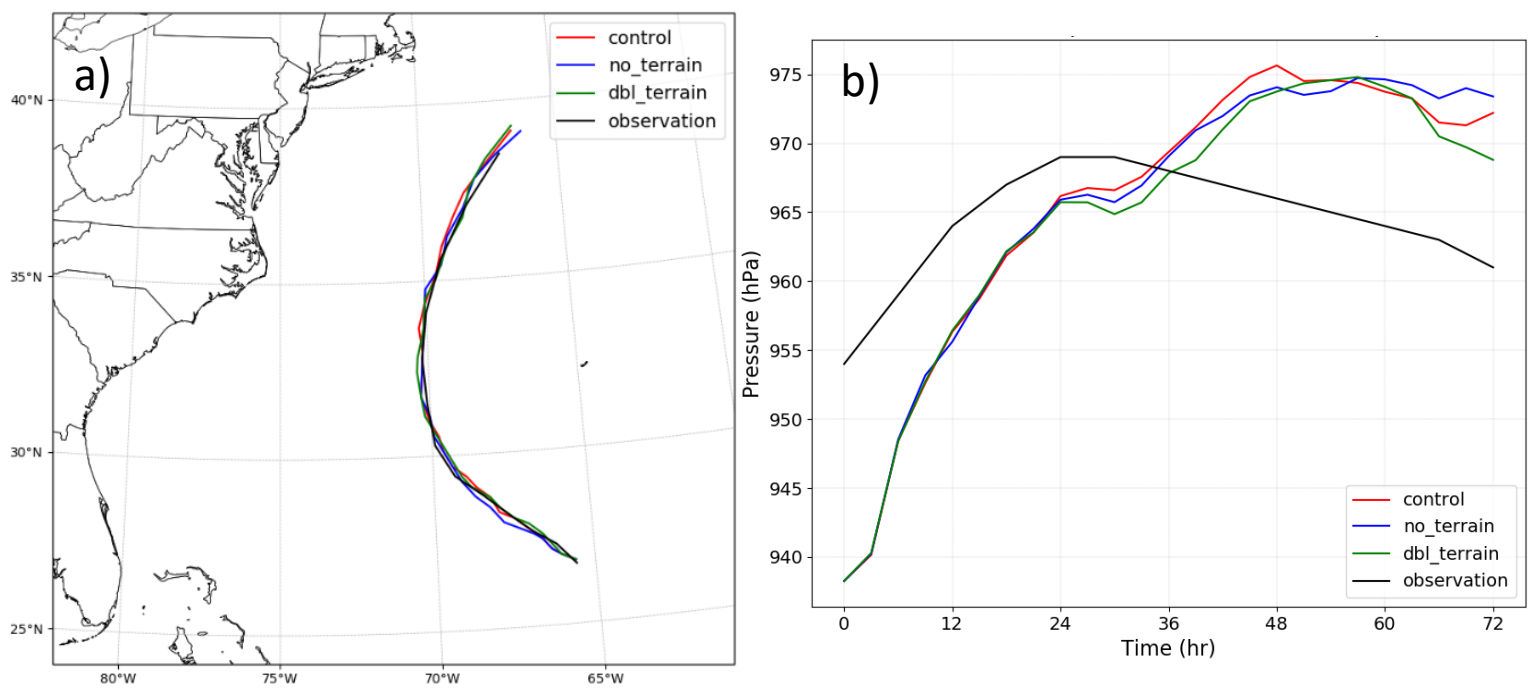

Figure A.3. Hurricane Katia a) tracks and b) central pressures from 1200 UTC 06 September 2011 to 1200 UTC 09 September 2011 resulting from the National Hurricane Center best track analysis (black), WRF d01 control simulation (red), and the experimental WRF d01 simulations with terrain removed (blue) and terrain doubled (green).

This is in part due to the WRF simulation accurately accounting for the track of Hurricane Katia (Fig. A.3a) and the strength of Hurricane Katia from 0000 UTC 07 September 2011 to 0000 UTC 08 September 2011 (Fig. A.3b).

The 2016 North Carolina flood was simulated from 1200 UTC 28 September 2016 to 0000 UTC 01 October 2016 using the domains found in Figure A.1b. The maximum rainfall area in the control simulation for North Carolina fell approximately $150 \mathrm{~km}$ east of the actual location during the event, but the interest value for this try was the highest at 0.91 (Fig. A.4).

Each event had a handful of test runs, except for the 2014 Islip, New York flood which required nearly ten tries for a realistic control simulation. The 2014 event was very sensitive to location of rainfall and no simulation was able to place the extreme rain over 


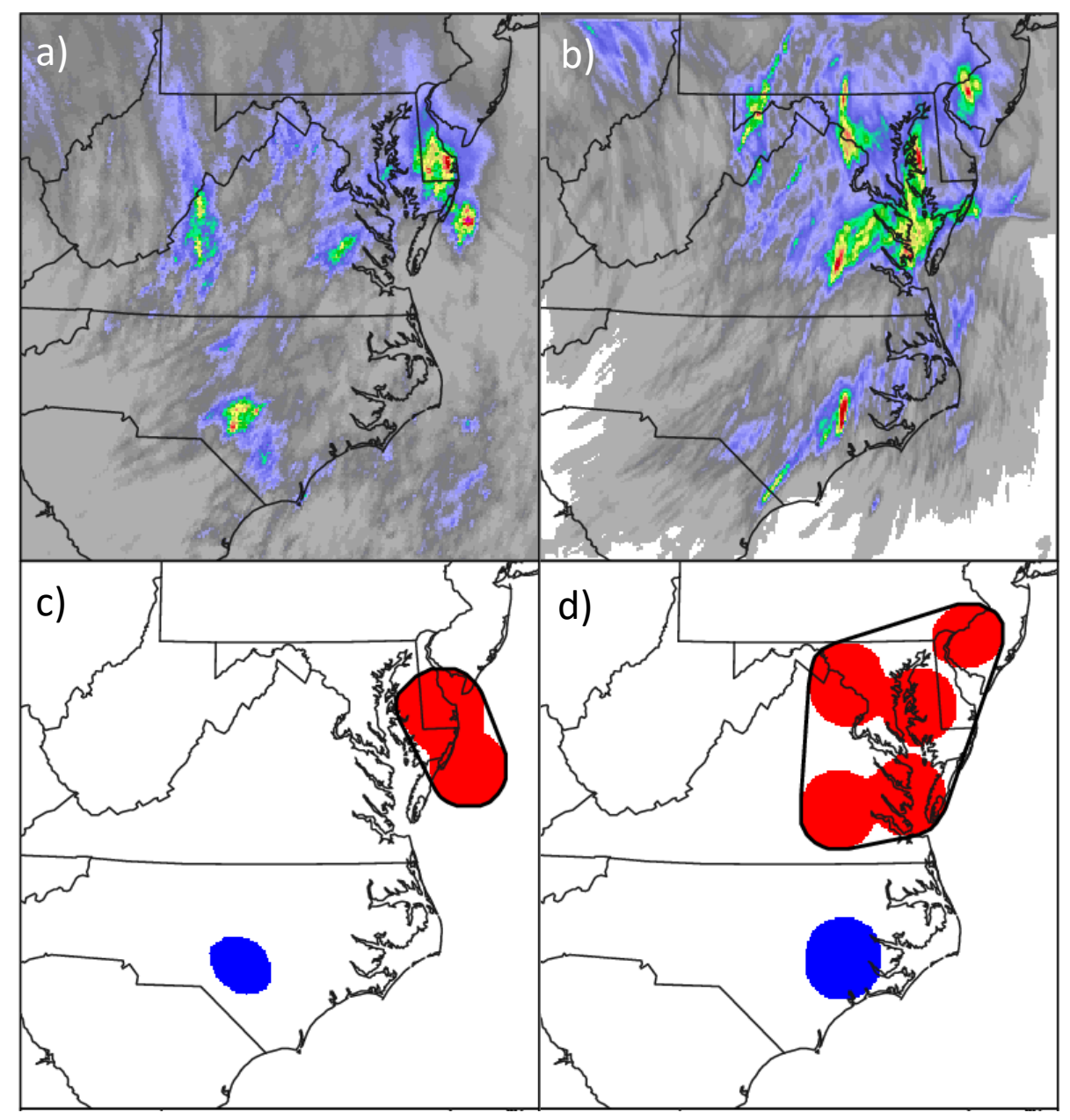

Figure A.4. Precipitation amount for the simulation of the North Carolina rain event from 1200 UTC 28 September 2016 to 0000 UTC 01 October 2016 created using a) 4-km NCEP Stage IV reanalysis data from Earth Observing Laboratory (EOL) and b) WRF control simulation output for domain 2. The MODE results are shown to identify an object (red) for c) the reanalysis data shown in a) and d) the WRF data shown in b) that are corresponding in both analyses based on a threshold of $254 \mathrm{~mm}$. The total interest value calculated in MODE for the objects (red) shown in c) and d) is 0.91 .

Long Island, New York. The most accurate result contained the domain configuration found in Figure A.1c and was initialized at 1200 UTC 12 August 2014, ending at 0600 UTC 14 August 2014. The maximum precipitation fell well off the coast and the matched objects verified with only a 0.85 interest value (Fig. A.5). Due to the high sensitivity, it 


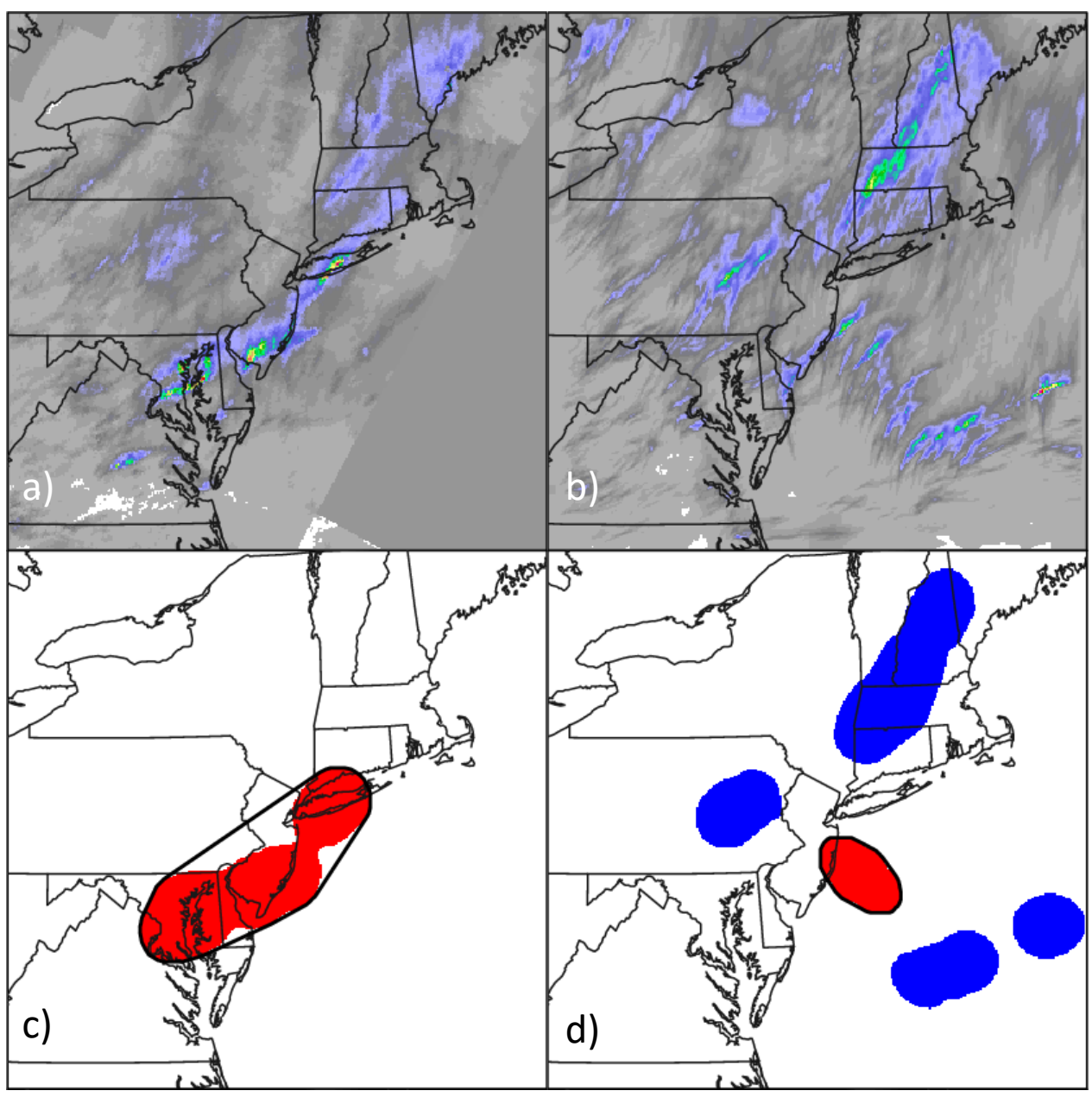

Figure A.5. Precipitation amount for the simulation of the Islip, New York heavy rain event from 1200 UTC 12 August 2014 to 0600 UTC 14 August 2014 created using a) 4-km NCEP Stage IV reanalysis data from Earth Observing Laboratory (EOL) and b) WRF control simulation output for domain 2. The MODE results are shown to identify an object (red) for c) the reanalysis data shown in a) and d) the WRF data shown in b) that are corresponding in both analyses based on a threshold of $127 \mathrm{~mm}$. The total interest value calculated in MODE for the objects (red) shown in c) and d) is 0.85 .

was decided to use this control simulation even though the interest value was below 0.90 , rather than complete an intense sensitivity test like that of the 2015 South Carolina flood event that required a considerable amount of time and resources. 


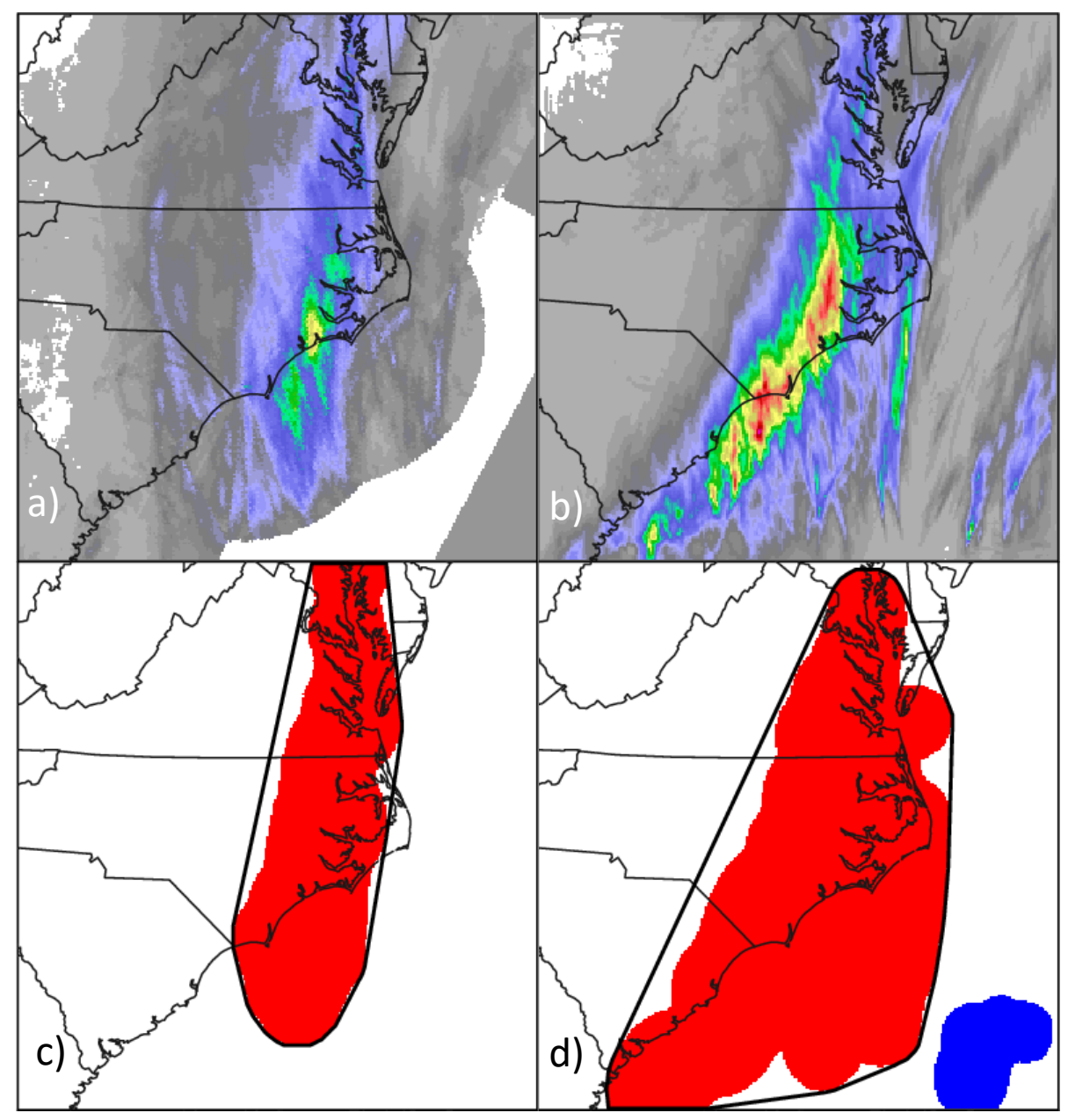

Figure A.6. Precipitation amount for the simulation of the North Carolina heavy rain event from 0000 UTC 29 September 2010 to 1800 UTC 01 October 2010 created using a) 4-km NCEP Stage IV reanalysis data from Earth Observing Laboratory (EOL) and b) WRF control simulation output for domain 2. The MODE results are shown to identify an object (red) for c) the reanalysis data shown in a) and d) the WRF data shown in b) that are corresponding in both analyses based on a threshold of $254 \mathrm{~mm}$. The total interest value calculated in MODE for the objects (red) shown in c) and d) is 0.94 .

The 2010 North Carolina flood was simulated from 0000 UTC 29 September 2010 to 1800 UTC 01 October 2010 using the domains seen in Figure A.1d. The only issue with this control simulation is the overestimation of precipitation, but MODE verified the entire precipitation field with an interest value of 0.94 (Fig. A.6). 


\section{APPENDIX B.}

For the 2015 South Carolina event, a synoptic analysis of irrotational flow was completed using CFSR data (Fig. 4.2). That analysis suggested latent heat release in the location of the intense precipitation to contribute to negative PV advection of the irrotational wind, slowing the propagation of the upper-level trough. All events in this study involved intense precipitation and an upper-level trough. Therefore, it was important to share CFSR results of irrotational flow for each event. All events had evidence of negative PV advection by the irrotational wind to some degree (Fig. B.1, B.2, B.3, B.4).

The 2010 and 2011 events most closely resembled the irrotational flow pattern of the 2015 event, with irrotational wind vectors oriented perpendicular to the upper-level jet between the region of ascent and the increased PV, suggesting negative PV advection (Fig. B.1, B.4). The 2010, 2011, and 2015 events lasted multiple days due to the stagnant synoptic environment, which was most likely caused by the positive feedback system of the intense precipitation and associated latent heat release acting against the upper-level flow. The 2014 and 2016 events involved intense precipitation over a period of hours, instead of days, so it was no surprise to find no clear influence from the region of ascent/precipitation on the upper-level PV/trough (Fig. B.2, B.3). 


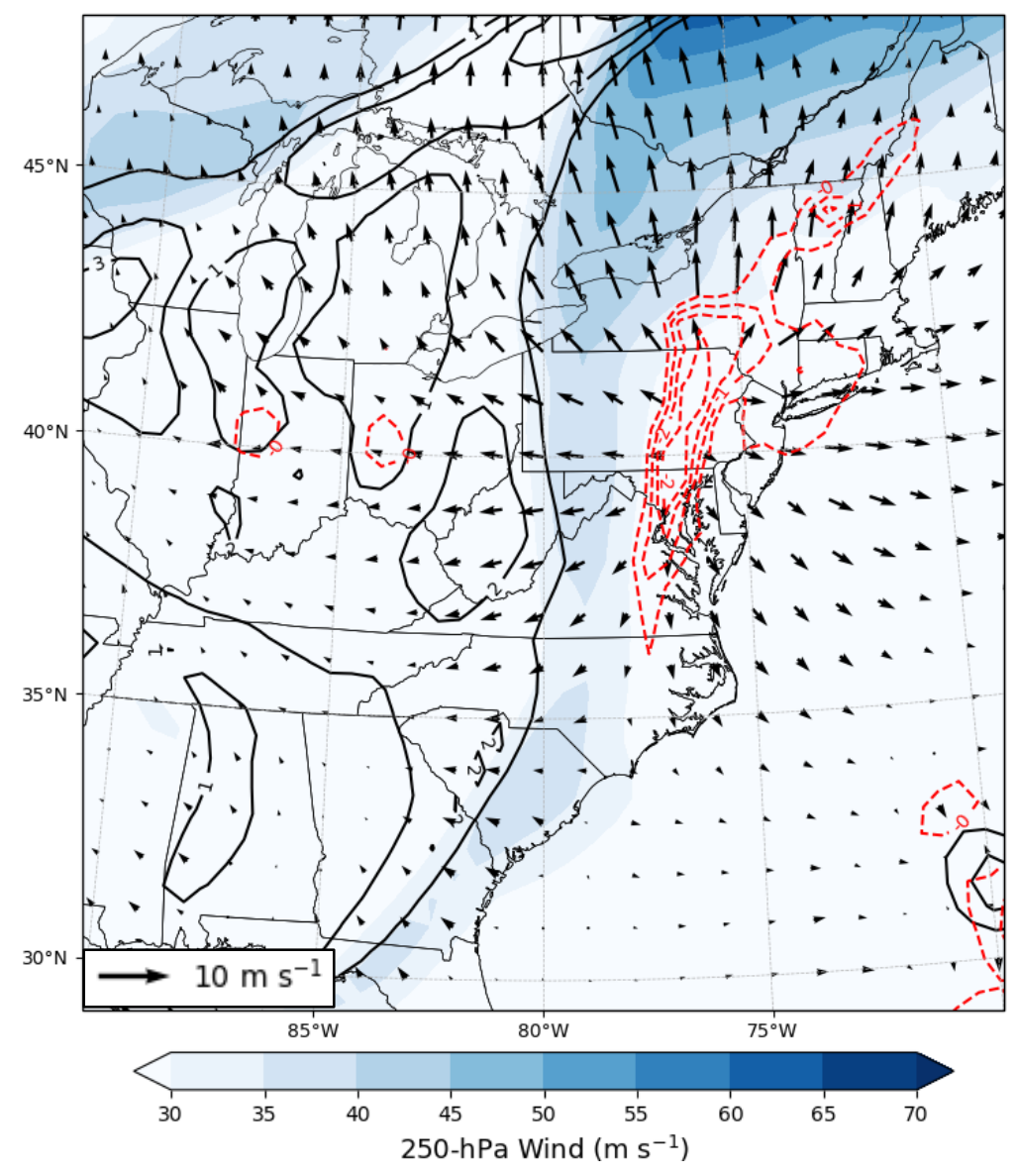

Figure B.1. Climate Forecast System Reanalysis (CFSR) valid at 0600 UTC 08 September 2011 representing 250-hPa wind ( $\mathrm{m} \mathrm{s}^{-1}$; shaded), 600-400-hPa ascent $\left(5 \times 10^{-3} \mathrm{hPa} \mathrm{s}^{-1}\right.$; dashed red), 300-200-hPa potential vorticity (PVU; contoured) and irrotational wind $\left(\mathrm{m} \mathrm{s}^{-1}\right)$. 


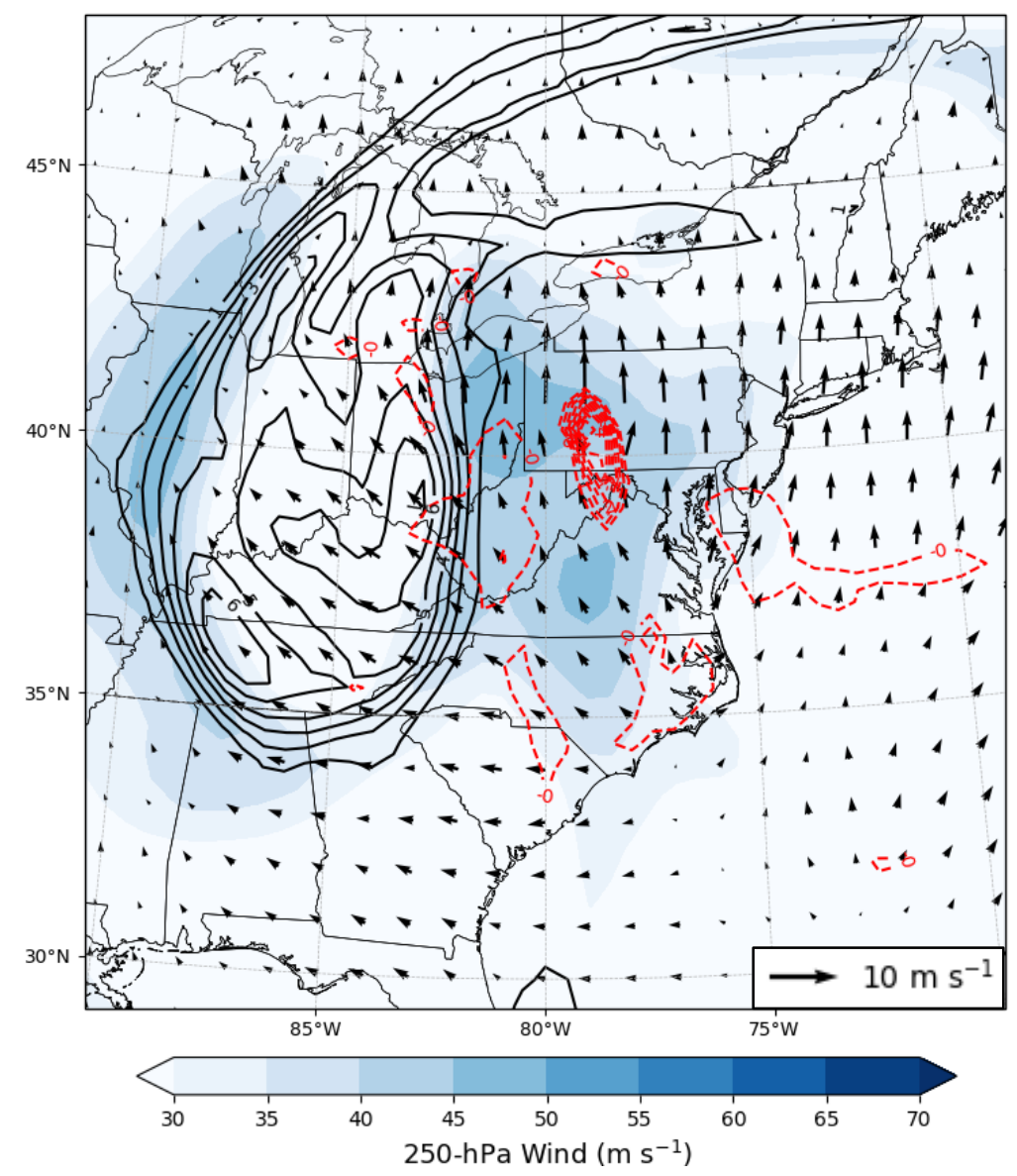

Figure B.2. As in Fig. B.1, except valid at 1800 UTC 29 September 2016. 


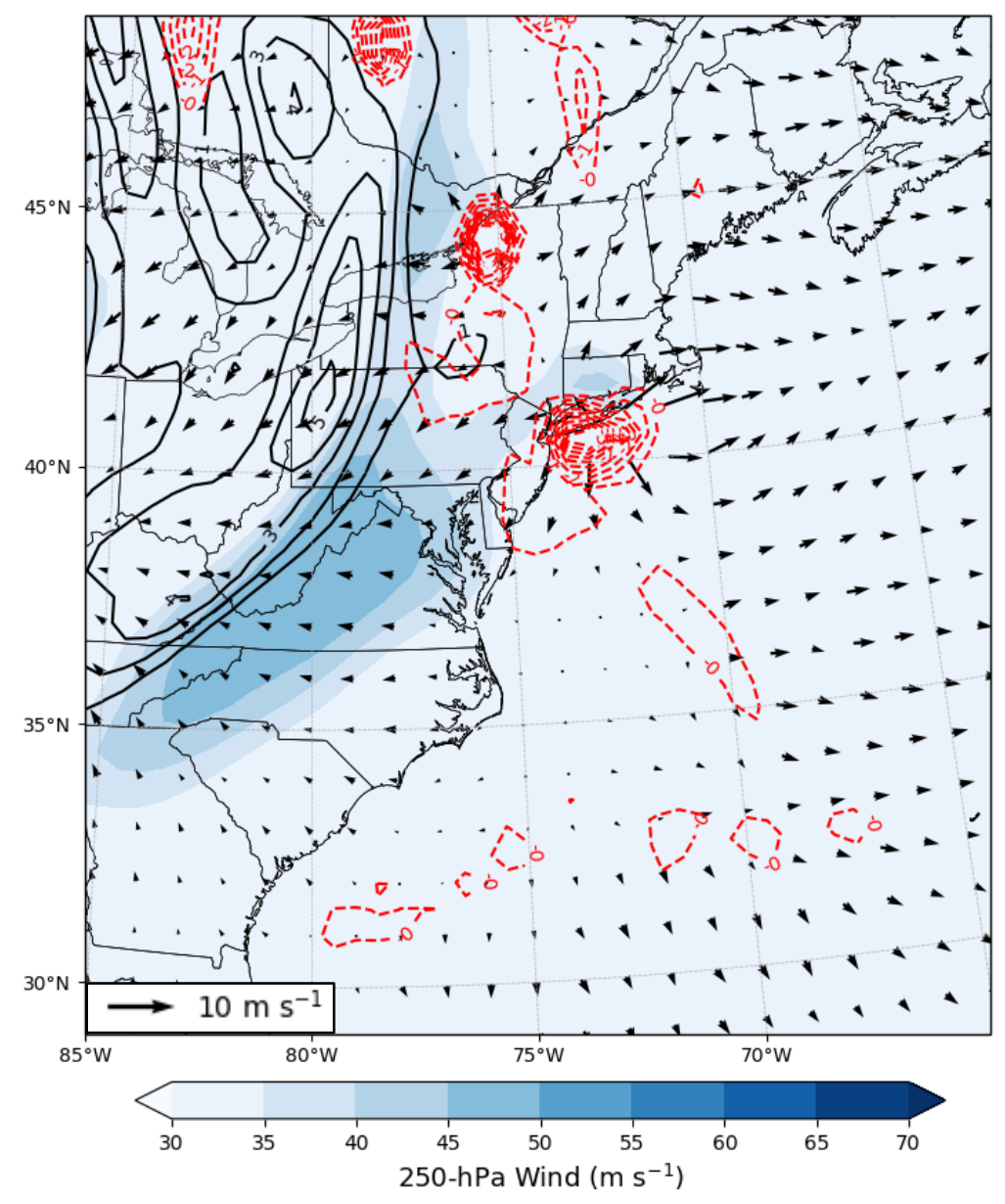

Figure B.3. As in Fig. B.1, except valid at 0600 UTC 13 August 2014. 


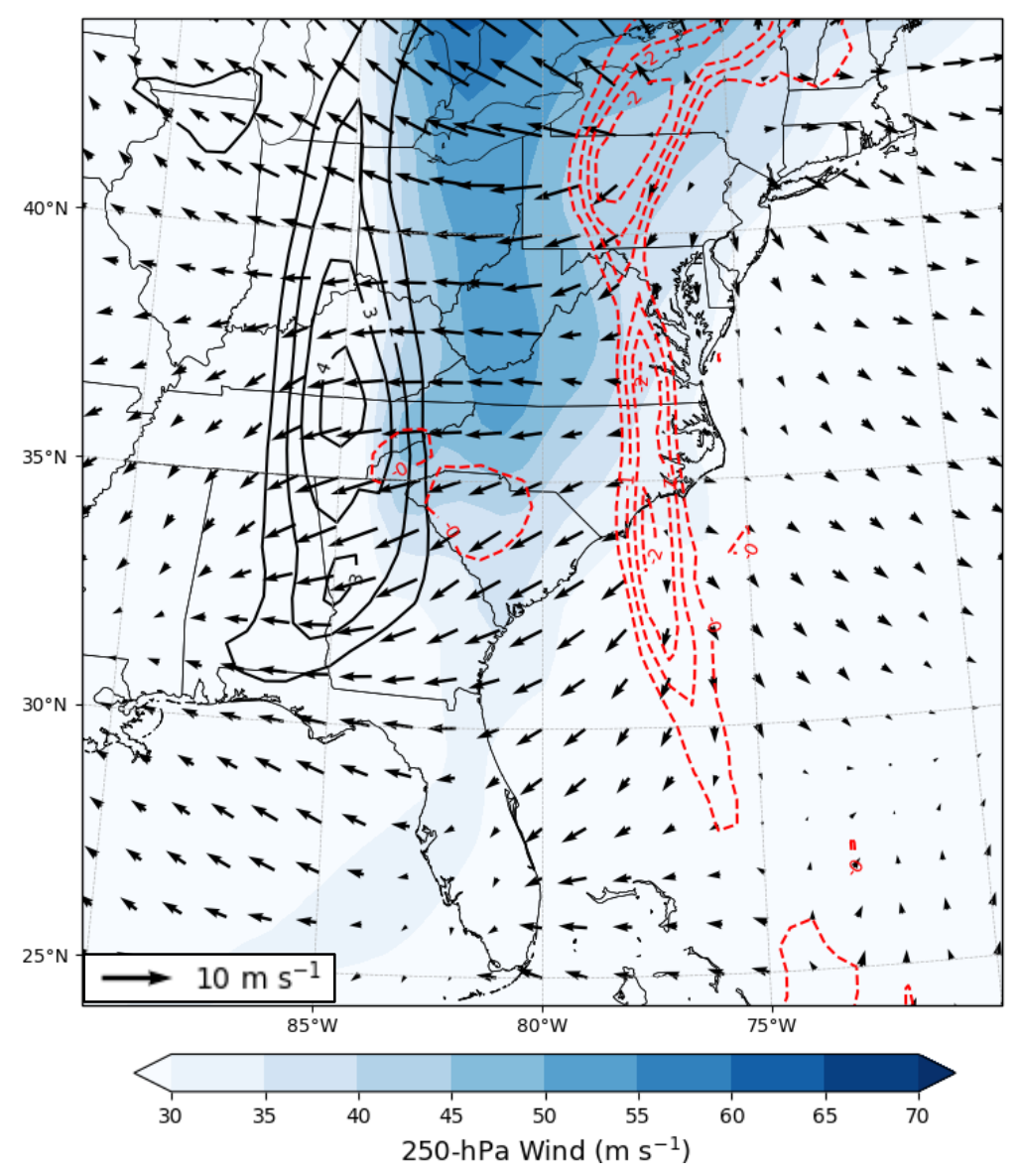

Figure B.4. As in Fig. B.1, except valid at 1800 UTC 30 September 2010. 


\section{REFERENCES}

Atallah, E. H., and L. F. Bosart, 2003: The extratropical transition and precipitation distribution of Hurricane Floyd (1999). Mon. Wea. Rev., 131, 1063-1081.

Beljaars, A.C.M., 1994: The parameterization of surface fluxes in large-scale models under free convection. Quart. J. Roy. Meteor. Soc., 121, 255-270.

Berg, R., 2016: Hurricane Joaquin. NHC TC Report/AL112015, 36 pp.

Bluestein, H., 1986: Fronts and jet streaks: A theoretical perspective. Mesoscale Meteorology and Forecasting, P. S. Ray, Ed., Amer. Meteor. Soc., 173-215.

Bosart, L. F., C. J. Vaudo, and J. H. Helsdon, Jr., 1972: Coastal frontogenesis. J. Appl. Meteor., 11, 1236-1258.

Bosart, L. F., 1975: New England coastal frontogenesis. Quart. J. Roy. Meteor. Soc., 101, 957-978.

Brown, M., 2012: Remnants of Tropical Storm Lee and the Susquehanna River Basin Flooding of September 6-10, 2011. NOAA Service Assessment. 64 pp. www.weather.gov/media/publications/assessments/LeeSusquehanna12.pdf

Bryan, G. H., and J. M. Fritsch, 2000: Moist Absolute Instability: The Sixth Static Stability State. Bull. Amer. Meteor. Soc., 81, 1207-1230.

Colle, B. A., 2003: Numerical simulations of the extratropical transition of Floyd (1999): Structural evolution and responsible mechanisms for the heavy rainfall over the Northeast United States. Mon. Wea. Rev., 131, 2905-2926.

Colman, B. R., 1990: Thunderstorms above frontal surfaces in environments without positive CAPE. Part I: A climatology. Mon. Wea. Rev., 118, 1103-1121.

Corfidi, S. F., S. J. Corfidi, and D. M. Schultz, 2006: Toward a better understanding of elevated convection. Preprints 23rd Conf. on Severe Local Storms, St. Louis, Mo, Amer. Meteor. Soc., CD-ROM P1.5.

Cote, M. R., 2007: Predecessor rain events in advance of tropical cyclones. M.S. thesis, Department of Atmospheric and Environmental Sciences, University at Albany, State University of New York, $200 \mathrm{pp}$. [Available online at http://cstar.cestm.albany.edu/CAP_Projects/Project10/index.htm].

Dale, J., and D. G. Lackmann, 2014: The Role of Hurricane Ernesto (2006) in a Predecessor Rainfall Event. 26th Conf. on Wea. Analysis and Forecasting, Atlanta, GA, Amer. Meteor. Soc. [Available online at https://ams.confex.com/ams/94Annual/webprogram/Paper236200.html] 
Davis C. A., B. G. Brown, R. G. Bullock, and J. Halley Gotway, 2009: The Method for Object-based Diagnostic Evaluation (MODE) applied to WRF forecasts from the 2005 NSSL/SPC Spring Program. Wea. Forecasting, 24, 1252-1267.

Doswell, C. A., 1987: The distinction between large-scale and mesoscale contribution to severe convection: a case study example. Wea. Forecasting, 2, 3-16.

Dyer, A. J., and B. B. Hicks, 1970: Flux-gradient relationships in the constant flux layer. Quart. J. Roy. Meteor. Soc., 96, 715-721.

European Centre for Medium-Range Weather Forecasts, 2011: ECMWF's Operational Model Analysis, starting in 2011. Research Data Archive at the National Center for Atmospheric Research, Computational and Information Systems Laboratory, Boulder, CO. [Available online at https://doi.org/10.5065/D6ZG6Q9F.] Accessed 22 June 2017.

FEMA, 2016: South Carolina Dam Failure Assessment and Advisement. FEMA P-1801. DR-SC-4241. 64 pp. www.fema.gov/media-library-data/1488655463993943cd4a8efe4232f52baba5bb9c92ae4/FEMA_P-1081.pdf

Fredrick, S., C. Davis, D. Gill, and S. Low-Nam, 2009: Bogussing of Tropical Cyclones in WRF Version 3.1. 6, National Center for Atmospheric Research, Boulder, CO.

Fujiwhara, S., 1931: Short note on the behavior of two vortices. Proc. Phys.-Math. Soc. Japan, 13, 106-110.

Galarneau, T. J., L. F. Bosart, and R. S. Schumacher, 2010: Predecessor rain events ahead of tropical cyclones. Mon. Wea. Rev., 138, 3272-3297.

Gitro, C. M., M. S. Evans, and R. H. Grumm, 2014: Two major heavy rain/flood events in the mid-Atlantic: June 2006 and September 2011. J. Operational Meteor., 2, 152-168.

Halcomb, C. E., and P. S. Market, 2003: Forcing, instability and equivalent potential vorticity in a Midwest USA convective snowstorm. Meteor. Appl., 10, 273-280.

Hanley, D., J. Molinari, and D. Keyser, 2001: A Composite Study of the Interactions between Tropical Cyclones and Upper-Tropospheric Troughs. Mon. Wea. Rev., 129, 2570-2584.

Henson, C. B., and P. S. Market, 2017: Factors influencing elevated convection during the Hurricane Joaquin (2015) event. $28^{\text {th }}$ Conference on Weather Analysis and Forecasting, Seattle, WA, Amer. Meteor. Soc., 25 January 2017, 8B.6.

Hong, S-Y., and J-O. J. Lim, 2006: The WRF single-moment 6-class microphysics scheme (WSM6). J. Korean Meteor. Soc., 42, 129-151.

Hong, S-Y., Y. Noh, J. Dudhia, 2006: A new vertical diffusion package with an explicit treatment of entrainment processes. Mon. Wea. Rev., 134, 2318-2341. 
Iacono, M. J., J. S. Delamere, E. J. Mlawer, M. W. Shephard, S. A. Clough, and W. D. Collins, 2008: Radiative forcing by long-lived greenhouse gases: Calculations with the AER radiative transfer models. J. Geophys. Res., 113, D13103.

Jones, S. C., and Coauthors, 2003: The extratropical transition of tropical cyclones: Forecast challenges, current understanding, and future directions. Wea. Forecasting, 18, 1052-1092.

Jurewicz, M. L., 2012: The historic heavy rainfall and flooding events of 7-8 September 2011 across the mid-Atlantic and Northeastern states. Preprints, $37^{\text {th }}$ Natl. Wea. Assoc. Annual Meeting, Madison, WI, Natl. Wea. Assoc., B5.2.

Kastman, J. S., L. D. McCoy, P. S. Market, and N. I. Fox, 2017: An example of synergistic coupling of upper- and lower-level jets associated with flash flooding. Meteor. Appl., 24, 206-210.

Kimball, S. K., and J. L. Evans, 2002: Idealized Numerical Simulations of HurricaneTrough Interaction. Mon. Wea. Rev., 130, 2210-2227.

Knutson, T. R., J. L. McBride, J. Chan, K. Emanuel, G. Holland, C. Landsea, I. Held, J. P. Kossin, A. K. Srivastava, and M. Sugi, 2010: Tropical cyclones and climate change. Nature Geoscience, 3, 157-163.

Ladwig, W., 2017: wrf-python. UCAR/NCAR, Boulder, CO. doi:10.5065/D6W094P1.

Lewis, B. M., and D. P. Jorgensen, 1978: Study of the dissipation of Hurricane Gertrude (1974). Mon. Wea. Rev., 106, 1288-1306.

Lin, Y., and K. E. Mitchell, 2004: The NCEP Stage II/IV Hourly Precipitation Analyses: Development and Applications. Environmental Modeling Center, National Centers for Environmental Prediction, Camp Springs, MD.

Lupo, A. R., and P. J. Smith, 1995: Planetary and synoptic-scale interactions during the life cycle of a mid-latitude blocking anticyclone over the North Atlantic. Tellus. 47A. 575-596.

Maddox, R. A., C. F. Chappell, and L. R. Hoxit, 1979: Synoptic and meso-alpha aspects of flash flood events. Bull. Amer. Meteor. Soc., 60, 115-123.

Marciano, C., and G. Lackmann, 2017: The South Carolina flood of October 2015: Moisture transport analysis and the role of Hurricane Joaquin. J. Hydrometeor, 18, 2973-2990. doi:10.1175/JHM-D-16-0235.1.

May, R. M., S. C. Arms, P. Marsh, E. Bruning, and J. R. Leeman, 2017: MetPy: A Python Package for Meteorological Data. Unidata. doi:10.5065/D6WW7G29.

McCoy, L. P., P. S. Market, C. M. Gravelle, C. E. Graves, N. I. Fox, S. M. Rochette, J. Kastman, and B. Svoma, 2017: Composites of heavy rain producing elevated thunderstorms in the Central United States. Adv. Meteor., 2017, 1-19.

Means, L. L., 1952: On thunderstorm forecasting in the central United States. Mon. Wea. Rev., 80, 165-189. 
Miller, J. E., 1948: On the concept of frontogenesis. J. Meteor., 5, 169-171.

Moore, B. J., P. J. Neiman, F. M. Ralph, and F. E. Barthold, 2012: Physical processes associated with heavy flooding rainfall in Nashville, Tennessee, and vicinity during 1-2 May 2010: The role of an atmospheric river and mesoscale convective systems. Mon. Wea. Rev., 140, 358-378.

Moore, J. T., A. C. Czarnetzki, and P. S. Market, 1998: Heavy precipitation associated with elevated thunderstorms formed in a convectively unstable layer aloft. Meteor. Appl., 5, 373-384.

Mudrick, S. E., 1974: A numerical study of frontogenesis. J. Atmos. Sci., 31, 869-892.

Mueller, M. J., K. M. Mahoney, and M. Hughes, 2017: High-resolution model-based investigation of moisture transport into the Pacific Northwest during a strong atmospheric river event. Mon. Wea. Rev., 145, 3861-3879.

Murphy, J. D., 2016: The Historic South Carolina Floods of October 1-5, 2015. NOAA Service Assessment. 113 pp. www.weather.gov/media/publications/assessments/SCFlooding_072216_Signed_ Final.pdf

Otto, R., 2010: Heavy Rains Across the Mid-Atlantic and Northeast. NOAA Event Summary. 14 pp.

https://www.wpc.ncep.noaa.gov/storm_summaries/event_reviews/2010/MidAtlantic_and_Northeast_Heavy_Rains_2010.pdf

Peng, M. S., R. N. Maue, C. A. Reynolds, and R. H. Langland, 2007: Hurricanes Ivan, Jeanne, Karl (2004) and mid-latitude trough interactions. Meteorol. Atmos. Phys., 97, 221-237.

Petterssen, S., 1936: Contribution to the theory of frontogenesis. Geo-fys. Publ., 11, 1-27.

Polissar, A. V., P. K. Hopke, and J. M. Harris, 2001: Source regions for atmospheric aerosol measured at Barrow, Alaska. Env. Sci. Tech., 35, 4214-4226.

Reges, H. W., N. Doesken, J. Turner, N. Newman, A. Bergantino, and Z. Schwalbe, 2016: CoCoRaHS: The Evolution and Accomplishments of a Volunteer Rain Gauge Network. Bull. Amer. Meteor. Soc., 97, 1831-1846, https://doi.org/10.1175/BAMS-D-14-00213.1.

Richwien, B. A., 1980: The damming effect of the southern Appalachians. Nat. Wea. Dig., 5, 2-12.

Rochette, S. M., and J. T. Moore, 1996: Initiation of an elevated mesoscale convective system associated with heavy rainfall. Wea. Forecasting, 11, 443-457.

Ross, R. J., and Y. Kurihara, 1995: A Numerical Study on Influences of Hurricane Gloria (1985) on the Environment. Mon. Wea. Rev., 123, 332-346.

Saha, S., and Coauthors, 2014: The NCEP Climate Forecast System Version 2. J. Climate, 27, 2185-2208, https://doi.org/10.1175/JCLI-D-12-00823.1. 
Schultz, D. M., and C. A. Doswell III, 1999: Conceptual models of upper-level frontogenesis in southwesterly and northwesterly flow. Quart. J. Roy. Meteor. Soc., 125, 2535-2562.

Schumacher, R. S., and C. A. Davis, 2010: Ensemble-Based Forecast Uncertainty Analysis of Diverse Heavy Rainfall Events. Wea. Forecasting, 25, 1103-1122.

Stauffer, D. R., and T. T. Warner, 1987: A numerical study of Appalachian cold-air damming and coastal frontogenesis. Mon. Wea. Rev., 115, 799-821.

Stewart, S. R., 2012: Hurricane Katia. NHC TC Report/AL122011, 20 pp.

Stoelinga, M. T., 2009: A Users' Guide to RIP Version 4.6: A Program for Visualizing Mesoscale Model Output. University of Washington. Seattle, WA.

Tang, Q., L. Xie, G. M. Lackmann, and B. Liu, 2013: Modeling the Impacts of the LargeScale Atmospheric Environment on Inland Flooding during the Landfall of Hurricane Floyd (1999). Adv. Meteor., 2013, 1-16.

Tewari, M., F. Chen, W. Wang, J. Dudhia, M. A. LeMone, K. Mitchell, M. Ek, G. Gayno, J. Wegiel, and R. H. Cuenca, 2004: Implementation and verification of the unified NOAH land surface model in the WRF model. 20th conference on weather analysis and forecasting/16th conference on numerical weather prediction, pp. $11-15$.

Tiedtke, M., 1989: A comprehensive mass flux scheme for cumulus parameterization in large-scale models. Mon. Wea. Rev., 117, 1779-1800.

Torn, R. D., 2017: A comparison of the downstream predictability associated with ET and baroclinic cyclones. Mon. Wea. Rev., 145, 4651-4672.

Trenberth, K. E., J. Fasullo, and L. Smith, 2005: Trends and variability in columnintegrated atmospheric water vapor. Climate Dynamics, 24, 741-758.

Wang. W., and Coauthors, 2017: WRF-ARW Version 3.9 Modeling System User's Guide. National Center for Atmospheric Research, Boulder, CO.

Wang, Y., Yu. Wang, and H. Fudeyasu, 2009: The Role of Typhoon Songda (2004) in Producing Distantly Located Heavy Rainfall in Japan. Mon. Wea. Rev., 137, 36993716.

Zhang, D.-L., and R.A. Anthes, 1982: A high-resolution model of the planetary boundary layer- sensitivity tests and comparisons with SESAME-79 data. J. Appl. Meteor., 21, 1594-1609.

Zhang, Chunxi, Yuqing Wang, and Kevin Hamilton, 2011: Improved representation of boundary layer clouds over the southeast pacific in ARW-WRF using a modified Tiedtke cumulus parameterization scheme. Mon. Wea. Rev., 139, 3489-3513. 


\section{VITA}

Chasity Brooke Henson began her graduate school career in atmospheric science at the University of Missouri (MU) in 2014, after completing a Bachelor of Science degree in physics at Missouri Southern State University (MSSU). Atmospheric science was always the focus of Chasity's educational and career goals. In high school, she focused on math and science courses to prepare her for college. After graduating from Carthage High School as valedictorian in 2010, Chasity was able to attend MSSU as an honors student-athlete. Chasity's hard work in and out of the classroom allowed her to obtain scholarships from the MSSU Honors Program and MSSU Softball via the National Collegiate Athletic Association (NCAA).

MSSU did not have an atmospheric science major, so Chasity obtained her degree in physics with a minor in math to continue her preparation for a graduate school focus of atmospheric science. Chasity was able to complete her MSSU Honors senior thesis on the climatology of tornadoes for the Joplin, Missouri area. With the encouragement of her undergraduate advisor, Dr. Daniel Marsh, Chasity presented her thesis research at the Missouri Academy of Science (MAS). After her presentation, she was approached by Dr. Patrick Market from MU. He asked her if she was interested in graduate school and she informed him of her application to his program at MU. Dr. Market returned to MU after MAS and found Chasity's application. Just a couple days later, Chasity received an offer from Dr. Market to join his program at MU in atmospheric science as a research assistant. She accepted and obtained her Master of Science two years later from MU in atmospheric science with a thesis on the impacts of climate variability on Missouri crop yields. After 
studying under Dr. Market for two years, Chasity found a new area of interest in heavy precipitation events. Specifically, she focused on intense precipitation events resulting from the interactions of multiple atmospheric features on multiple scales along the East Coast of the United States as she continued her education towards a Doctor of Philosophy degree in atmospheric science at MU with Dr. Market as her advisor.

Throughout her Ph.D. career, Chasity was able to attend and present research at several national conferences for atmospheric science. Her research made it into several special sessions at conferences for the American Meteorological Society (AMS) and the National Weather Associate (NWA), but her highest award was first place for graduate student presentations at the 2017 NWA annual meeting in California. Chasity was also selected for participation in several graduate student opportunities with AMS, the National Center for Atmospheric Research (NCAR), and an internship with the Naval Research Laboratory (NRL) as a Ph.D. student at MU. Chasity found her participation in those opportunities to be vital to her education. She gained valuable skills in numerical weather prediction and knowledge in various meteorological phenomena that she utilizes in her career as a professional scientist at ENSCO, Inc. Chasity began her career at ENSCO, Inc. shortly before finishing her Ph.D., where she works with numerical weather prediction models in support of projects that protect the national security of the United States of America. After graduating with her Ph.D. in December 2019, Chasity plans to enjoy the life she has worked so hard to make for herself. 